\section{Pacific Northwest}

National Laboratory

Operated by Battelle for the

U.S. Department of Energy

\section{Hazard Analysis for the \\ Pretreatment Engineering Platform (PEP)}

June 2008

WE Lawrence

J Young

RS. Sullivan
JGH Geeting 


\title{
Hazard Analysis for the Pretreatment Engineering Platform (PEP)
}

\author{
R. S. Sullivan \\ J. G. H. Geeting \\ W. E. Lawrence \\ J. Young
}

June 2008

Prepared for

the U.S. Department of Energy

under Contract DE-AC05-76RL01830

Pacific Northwest National Laboratory

Richland, WA 99354 


\section{Summary}

The Pretreatment Engineering Platform (PEP) is designed to perform a demonstration on an engineering scale to confirm the Hanford Waste Treatment Plant Pretreatment Facility (PTF) leaching and filtration process equipment design and sludge treatment process. The system will use scaled prototypic equipment to demonstrate sludge water wash, caustic leaching, oxidative leaching, and filtration. Unit operations to be tested include pumping, solids washing, chemical reagent addition and blending, heating, cooling, leaching, filtration, and filter cleaning. In addition, the PEP will evaluate potential design changes to the ultrafiltration process system equipment to potentially enhance leaching and filtration performance as well as overall pretreatment throughput. The skid-mounted system will be installed and operated in the Processing Development Laboratory-West at Pacific Northwest National Laboratory (PNNL) in Richland, Washington.

This document identifies potential industrial safety, environmental, and cost impacts associated with the PEP and the safeguards needed to provide protection against them. This information has been produced by performing a series of hazard analyses at the 30\%,90\%, and $100 \%$ designs for the PEP. The hazard analyses address the PEP process equipment, intended operating activities, and anticipated maintenance and testing.

Three hazard analysis methodologies were used during this process, a Hazard Identification Checklist to identify all of the energy sources and material-at-risk present at and near the facility, a Hazard and Operability (HAZOP) study to address the process design, and a Preliminary Hazards Analysis (PHA) to address process activity integration with Processing Development Laboratory-West. Several recommendations were generated during the hazard analyses studies. These recommendations fell into three categories:

- Develop additional information on the design, operation, or maintenance of PEP to support completion of the hazard analysis.

- Perform engineering analyses to confirm design assumptions or bases.

- Provide additional safeguards not identified in the 30\% design package or intended operating scheme.

All recommendations were reviewed/resolved by the project by the time the final $100 \%$ design analysis sessions were complete. 


\section{Acronyms}

$\begin{array}{ll}\text { BNI } & \text { Bechtel National Inc. } \\ \text { CXP } & \text { cesium ion exchange process } \\ \text { FEP } & \text { feed evaporator producer } \\ \text { FRP } & \text { feed receipt vessel } \\ \text { HAZOP } & \text { Hazard and operability study } \\ \text { HLW } & \text { high-level waste } \\ \text { LAW } & \text { low-activity waste } \\ \text { MAR } & \text { material at risk } \\ \text { PDL-W } & \text { Processing Development Laboratory-West } \\ \text { PEP } & \text { Pretreatment Engineering Platform } \\ \text { PHA } & \text { Preliminary Hazard Analysis } \\ \text { PJM } & \text { pulse jet mixers } \\ \text { PNNL } & \text { Pacific Northwest National Laboratory } \\ \text { PTF } & \text { Hanford Pretreatment Facility } \\ \text { TDS } & \text { total dissolved solids } \\ \text { TKS } & \text { Tessenderlo Kerley Services } \\ \text { TMP } & \text { transmembrane pressure } \\ \text { UFP } & \text { ultrafiltration feed preparation } \\ \text { WGI } & \text { Washington Group Inc. } \\ \text { WTP } & \text { Waste Treatment Plant }\end{array}$





\section{Contents}

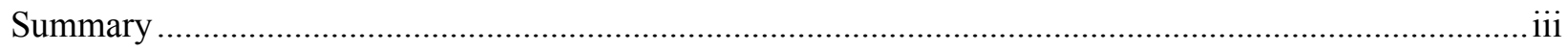

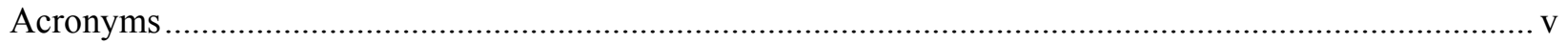

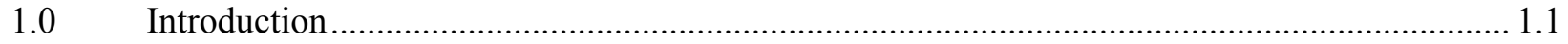

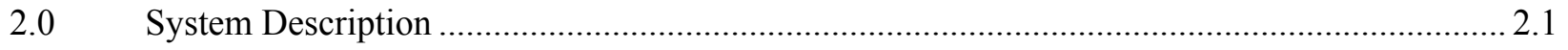

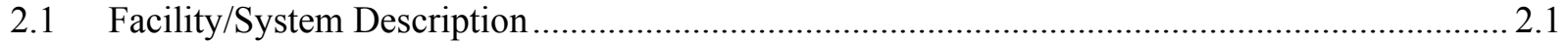

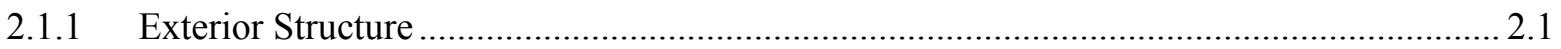

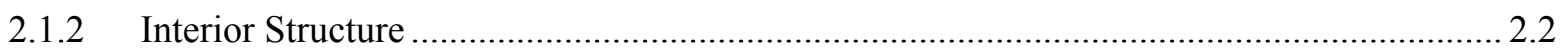

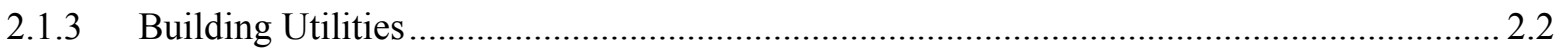

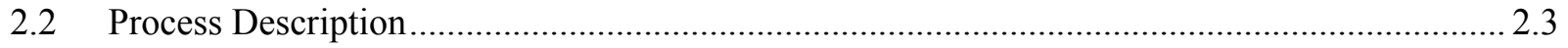

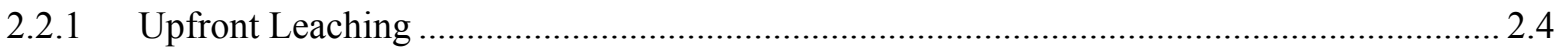

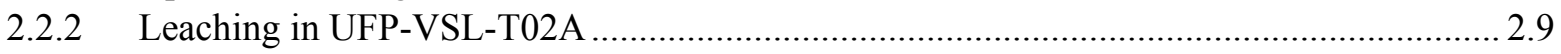

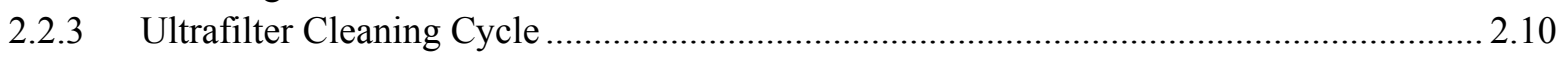

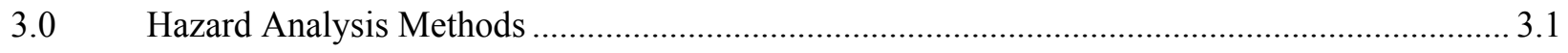

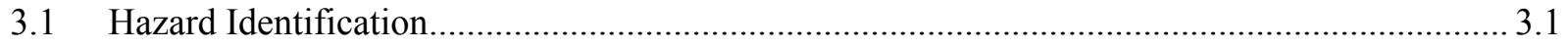

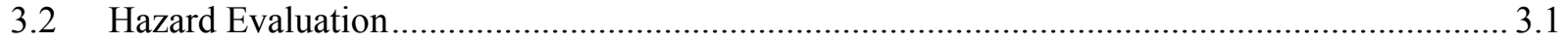

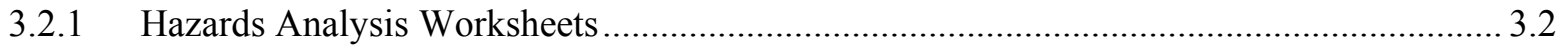

3.2.2 Likelihood Category Definitions.................................................................................... 3.3

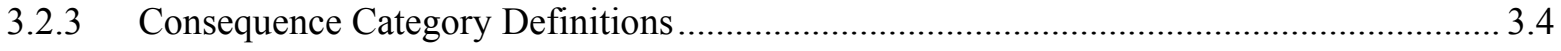

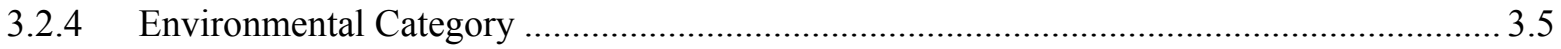

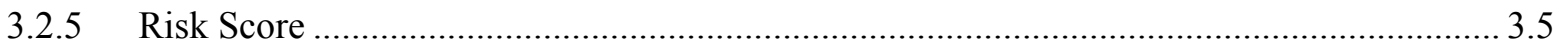

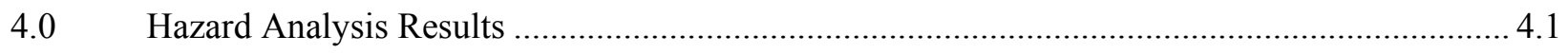

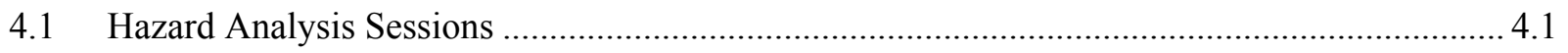

4.2 Hazard Identification and MAR Definition .................................................................... 4.2

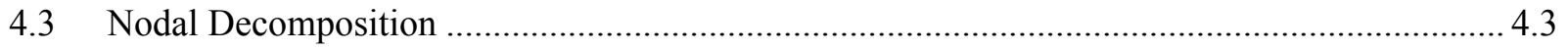

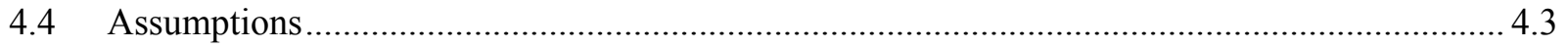

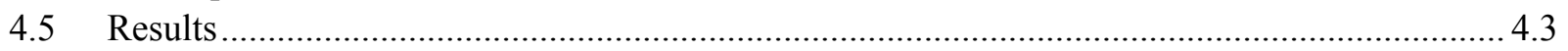

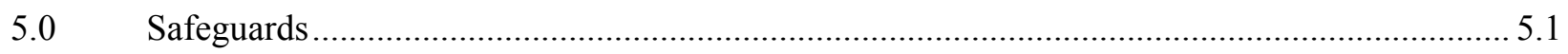

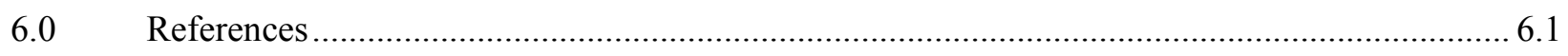

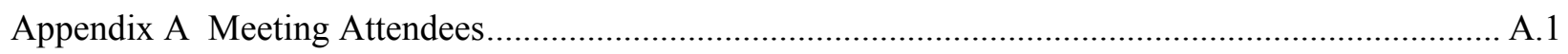

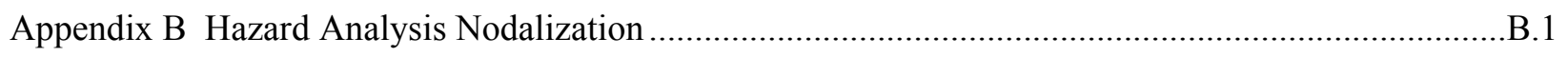

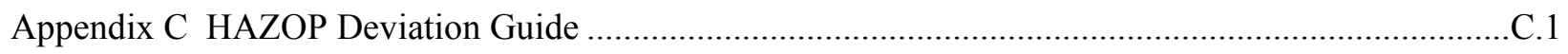

Appendix D Hazard Identification Checklist ................................................................................ D.1

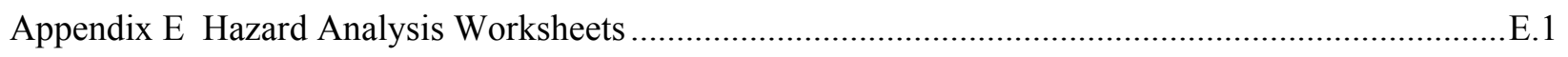

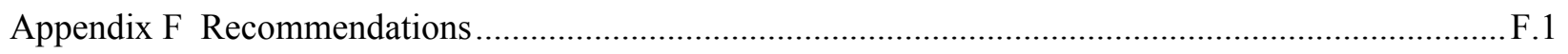

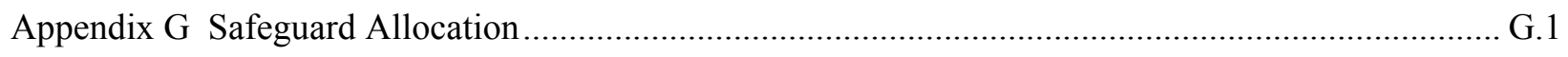

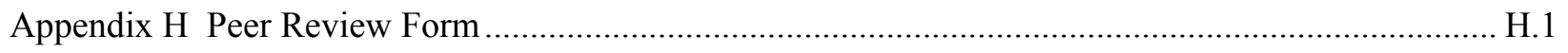




\section{Figures}

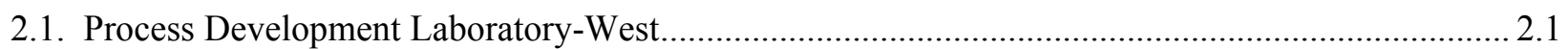

2.2. Simplified Flow Diagram of Engineering Scaled Pretreatment System ........................................ 2.5

2.3. Normal Operation of Ultrafilter Module and Permeate Process Diagram ..................................... 2.12

\section{Tables}

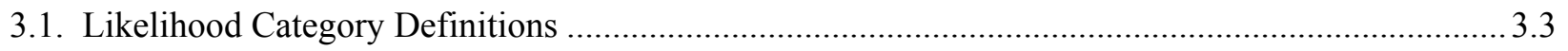

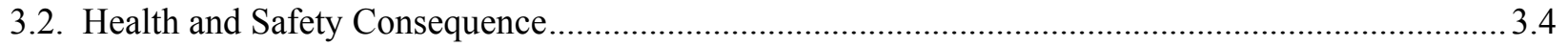

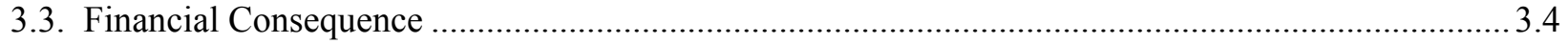

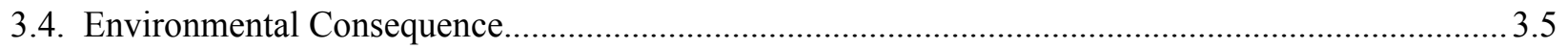

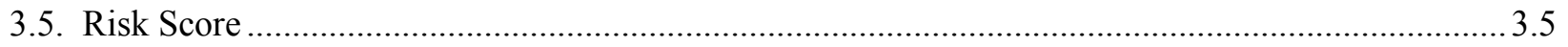

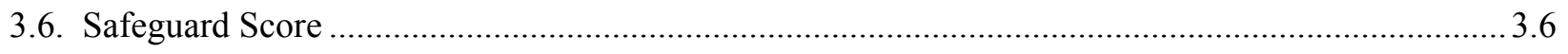

4.1. Hazard Analysis Assumptions/Ground Rules ................................................................................4

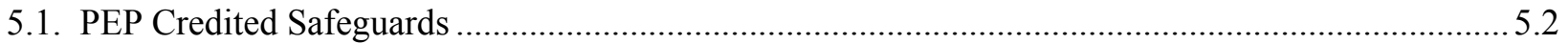




\subsection{Introduction}

This document identifies potential industrial safety, environmental, and cost impacts associated with the Hanford Waste Immobilization and Treatment Plant (WTP) Pretreatment Engineering Platform (PEP) and the safeguards needed to provide protection against them. The skid-mounted system will be installed and operated in the Processing Development Laboratory (PDL)-West facility at Pacific Northwest National Laboratory (PNNL) in Richland, Washington. This information has been produced by performing a series of hazard analyses at the $30 \%, 90 \%$ and $100 \%$ designs of the PEP (TKS 2007a,b,c). The purpose is to support the evolving design of the process equipment and development of operating and maintenance procedures for PEP as it will be operated and maintained in PDL-West.

The hazard analyses address the PEP process equipment, intended operating activities and anticipated maintenance and testing. The analyses consider the integration of PEP in PDL-West. Potential industrial safety issues, environmental releases, and cost impacts are addressed in the analyses. The cost of shutdown of PEP due to the need to replace major process system hardware is not addressed in the assessment of cost impacts. The analyses results included 1) identification of safeguards to manage safety; 2) recommendations to reduce risk, environmental releases, or costs; and/or 3) verification of design and operating assumptions. All recommendations were reviewed/resolved by the project by the time the $100 \%$ design analysis sessions were complete.

The PEP is designed to perform a demonstration on an engineering scale to confirm the Hanford Pretreatment Facility (PTF) leaching and filtration process equipment design [ultrafiltration feed preparation (UFP) system] and sludge treatment process flow sheet. The system will be designed using scaled prototypic equipment to demonstrate sludge water wash, caustic leaching, oxidative leaching, and filtration. The unit operations to be tested include pumping, solids washing, chemical reagent addition and blending, heating, cooling, leaching, filtration, and filter cleaning. In addition, the PEP will evaluate potential design changes to the UFP system leaching and ultrafiltration equipment as determined from engineering studies to potentially enhance leaching and filtration performance as well as overall pretreatment throughput. The skid-mounted system will be installed into the PDL-W. 


\subsection{System Description}

\subsection{Facility/System Description}

The PDL-W Building is a 6,826-square-foot building built in 1981 to provide high-bay space for hightemperature, high-pressure research on the expansion of metals by the PNNL Materials Group. It consists primarily of office and laboratory areas. The PDL-W building layout is shown in Figure 2.1.

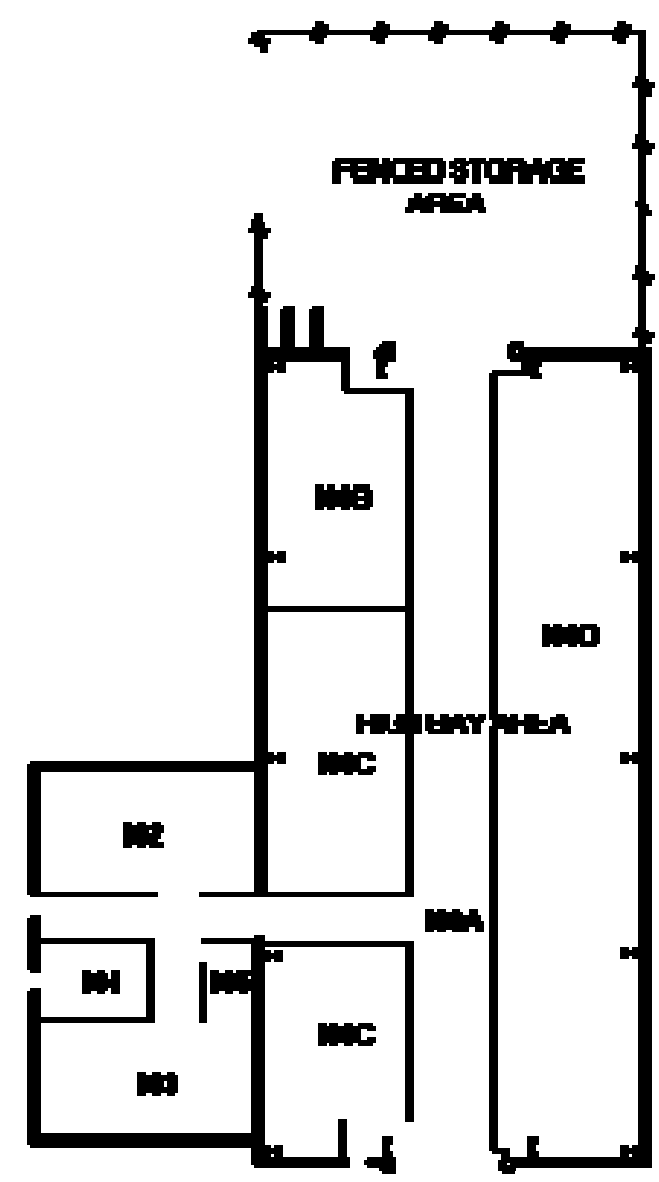

Figure 2.1. Process Development Laboratory-West

\subsubsection{Exterior Structure}

This facility is primarily a high-bay structure constructed on concrete foundations and concrete slab-ongrade floors. The building is steel framed and covered with prefabricated, insulated, galvanized steelribbed panels. It has a built-up flat roof. The high-bay area contains four load cells that are shrouded and 
capable of maintaining high temperatures and pressures. Connected to the high bay is a smaller building of similar structure that includes dry laboratories, a mechanical equipment room, and a restroom.

\subsubsection{Interior Structure}

The interior space where offices and lobby are located is constructed of gypsum wallboard with suspended ceiling tiles. The east wall of the office area is constructed of painted concrete blocks. There are $12 \times 12$-inch floor tiles in the hallway and vinyl sheeting material in the restrooms; offices have carpeting on the floors. Where rooms have been carpeted, the carpet may have been laid over the tile.

The high-bay area has a painted concrete floor and metal insulated walls and ceiling. The west wall is painted concrete block.

\subsubsection{Building Utilities}

The electric power capacity is supplied by one 1000-kVA City of Richland transformer. The main electrical panel is a 750-amp, 277/480-V, 3-phase, 4-wire service. A 400-amp, 277/480-V, 3-phase breaker services the east buss duct, and a 400-amp, 277/480-V, 3-phase breaker services the west buss duct. There is $120 / 208-\mathrm{V}$ service throughout the entire building and $480-\mathrm{V}$ to the high bay. There is no backup/standby power.

The HVAC for the building consists of the following:

- The heating capacity for the office area is supplied by a gas-fired, 2000-cfm, 92,000-Btu/hr heating capacity, 5.5-ton heat pump with a three-stage, 20-kW heating coil, 68,240 Btu/hr. The high bay has two 250,000 Btu/hr wall heaters.

- The cooling for the office area is supplied by a $2000-\mathrm{cfm}, 57,000 \mathrm{Btu} / \mathrm{hr}$ cooling capacity, 5.5-ton heat pump. The high bay has two 10-ton Trane air-conditioning units of $120,000 \mathrm{Btu} / \mathrm{hr}$ each.

- HVAC setback schedule for the office and high bay is Monday through Friday, 6:00 pm to 5:00 am and all weekend.

- The standard temperature control range (non-setback) is $60^{\circ}$ to $80^{\circ} \mathrm{F}$.

- There are 6 air exchanges per hour for the office area; for the high bay there are 0 to 10 .

Process and sanitary water is supplied by a 3-inch main line from the City of Richland. Two backflow preventers are provided with the water service.

- Process water is supplied at 200 gpm maximum.

- There is no reverse osmosis or deionized water capacity.

There is no laboratory manifold gas supply and capacity.

- Laboratory compressed air is delivered at $100 \mathrm{psi}$ from a $10 \mathrm{hp}$ air compressor with a $30 \mathrm{scfm}$ capacity.

- No laboratory vacuum capacity exists. 
Fire Zones: A Fire Zone "Control Area" is a building or portion of a building within which the exempted amounts of hazardous materials are allowed to be stored, dispensed, used, or handled, or which may provide extended fire protection to unique and/or valuable equipment.

Areas of coverage:

Zone $1 \quad 102,103,104,105,106 \mathrm{~A}, 106 \mathrm{~B}, 106 \mathrm{C}, 106 \mathrm{D}$, high bay

Zone $5 \quad$ PRMTR (areas within $5 \mathrm{ft}$ of bldg)

Zone $6 \quad$ OUTSD (areas outside $5 \mathrm{ft}$ of bldg)

\begin{tabular}{llc} 
Zone & Occupancy Class & Sprinklers (Yes/No) \\
\hline Zone 1 & Dry Laboratory ${ }^{(\text {a) }}$ & Yes \\
Zone 5 & Outside Storage & No \\
Zone 6 & Outside Storage & No
\end{tabular}

(a) The PDL-W is a high-bay building that involves pilot-scale research projects. This occupancy is unique in that it is not suitable for wet chemistry laboratory operations. However, the research performed in the building can involve larger quantities of hazardous materials due to the scale of the projects. All projects incorporate engineering controls equivalent to those provided in wet chemistry occupancies for the hazard of the materials involved.

Air and liquid effluent control systems present are the following:

- Air: None (ventilation for HVAC in the high bay will be increased to approximately $40,000 \mathrm{cfm}$ and will be handled by one or two variable-speed fans mounted on the roof).

- Liquid: Sanitary sewer system

Floor loading for the building consists of:

- Offices, restrooms: $100 \mathrm{lb} / \mathrm{ft}^{2}$

- Corridors, lobby, stairways: $100 \mathrm{lb} / \mathrm{ft}^{2}$

- Labs: $250 \mathrm{lb} / \mathrm{ft}^{2}$

Secondary containment: The whole high-bay floor was modified to serve as a secondary containment system.

Natural gas: The natural gas supply is approximately $3,000,000 \mathrm{Btu} / \mathrm{hr}$.

\subsection{Process Description}

The following sections provide a description of the normal process operation and major equipment with an explanation of functions and required plant services. The process description is derived from the Performance Requirements for Engineering Scale Pretreatment System (24590-PTF-3YD-UFP-00002 Rev. 1). Figure 2.2 is a simplified flow diagram of the UFP system with its associated components. 


\subsubsection{Upfront Leaching}

\subsubsection{High-Level Waste Simulant Receipt Vessel, HLP-VSL-T22}

The HLW receipt vessel receives HLW simulant. This vessel also acts as the "feed forward" vessel and may receive LAW simulant and evaporator recycles from FRP-VSL-T01 and FEP-VSL-T01, respectively. The feed is fed forward to UFP-VSL-T01A or B. For flexibility, the vessel contents can be recycled to FEP-VSL-T01.

The vessel is equipped with a variable frequency drive mechanical agitator to mix the simulant. The energy of the mixing can be varied to characterize the simulant as homogeneous or stratified, depending on the test requirements. The vessel will be sampled before any transfers out of the vessel are performed. Sampling may include determining requirements for leaching, amount of caustic to add for leaching (which can be added in-line during transfers), and the ratio of HLW to LAW feed simulant to blend in UFP-VSL-T01A/B.

The vessel has the capability to heat the simulant. The purpose of the heating function is to simulate the waste feed evaporator holdup time and upfront leaching. The waste feed evaporator is expected to be operated at approximately $55^{\circ} \mathrm{C}$; therefore, this vessel is equipped with a heating jacket. The vessel is equipped with temperature, level, and pressure measurements to monitor process conditions. The contents of the vessel are transferred out of the vessel by a pump that is equipped with a variable frequency drive. The contents can be transferred to UFP-VSL-T01A/B or FEP-VSL-T01. The vessel is also equipped with a bottom drain and is piped to UFP-VSL-T62A for evaporation considerations. 


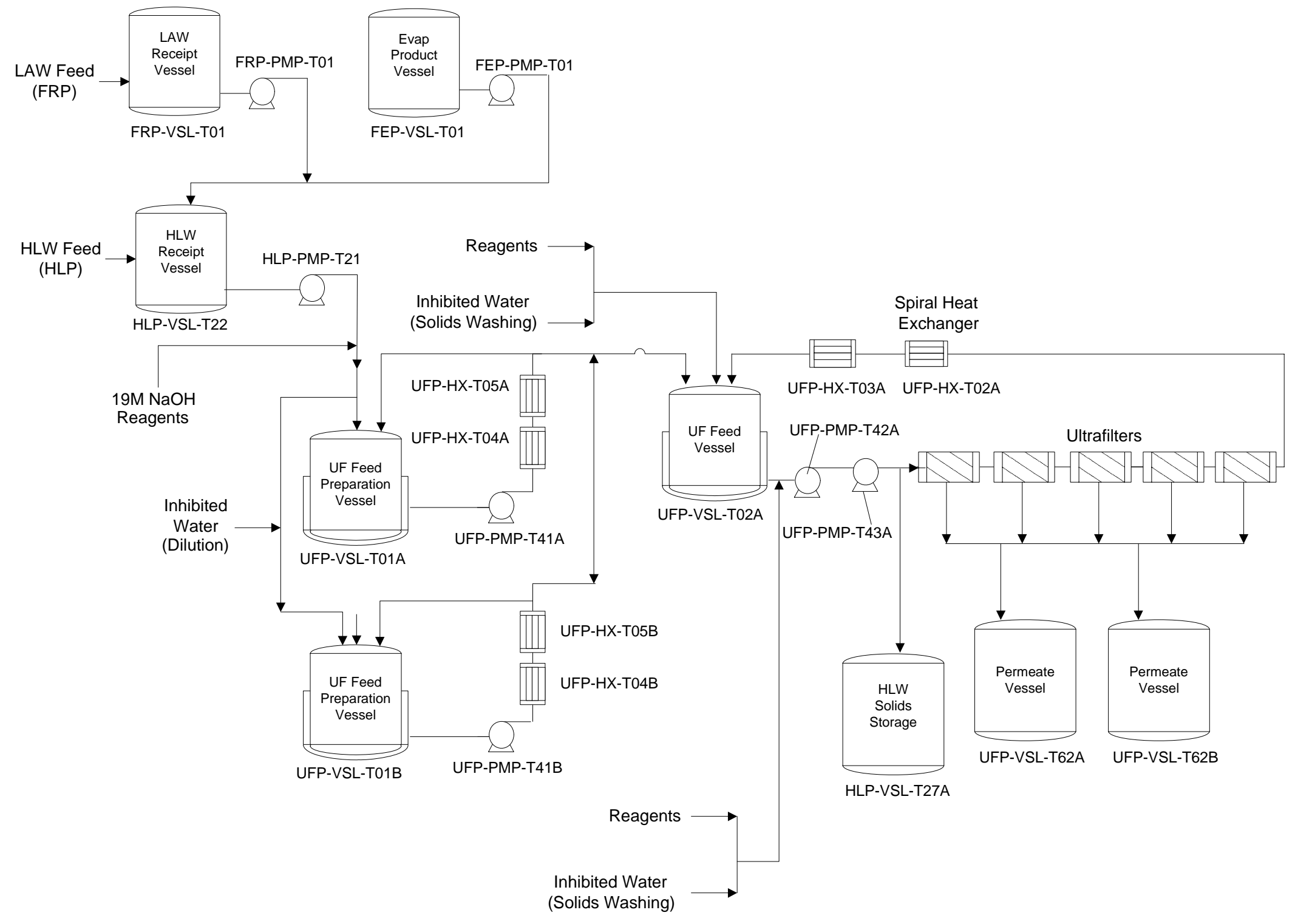

Figure 2.2. Simplified Flow Diagram of Engineering Scaled Pretreatment System 


\subsubsection{LAW Simulant Receipt Vessel, FRP-VSL-T01}

The LAW receipt vessel receives LAW simulant and may also receive evaporator recycles from FEP-VSL-T01. The vessel is equipped with a variable frequency drive mechanical agitator to mix the simulant. The energy of the mixing can be varied to characterize the simulant as homogenous or stratified, depending on the test requirements. The vessel will be sampled before any transfers out of the vessel are performed. Sampling may include determining requirements for leaching, amount of caustic to add for leaching, and the ratio of HLW to LAW feed simulant to blend in UFP-VSL-T01A/B. The vessel is designed to provide the same design functions as HLP-VSL-T22, with the capability to add a steam jacket in the future. The vessel is equipped with temperature, level and pressure measurements to monitor process conditions. The contents of the vessel are transferred out of the vessel by a pump that is equipped with a variable frequency drive. The contents can be transferred to HLP-VSL-T22 or to FEP-VSL-T01. The vessel is also equipped with a bottom drain which may be sent to UFP-VSL-T62A for evaporation considerations.

\subsubsection{Feed Evaporator Producer Product Vessel, FEP-VSL-T01}

The FEP product vessel is used as a backup feed receipt vessel and as a recycle hold vessel to provide extra lag storage when required. Recycles received in this vessel may include solids washing permeate, leach permeate, concentration permeate and various rinse water or spent cleaning reagents. The vessel may receive LAW simulant from FRP-VSL-T01, HLW simulant from HLP-VSL-T22 and recycles from UFP-VSL-T62B. The vessel will be designed to provide the same design functions as HLP-VSL-T22, with the capability to add a steam jacket in the future. The vessel is equipped with a variable frequency drive mechanical agitator to mix the simulant. The energy of the mixing can be varied to characterize the simulant as homogenous or stratified depending on the test requirements. The vessel will be equipped with sampling capabilities. Sampling may include determining requirements for leaching, amount of caustic to add for leaching, and the ratio of HLW to LAW feed simulant to blend in UFP-VSL-T01A/B. Sampling may also be used for recycle solution to determine post precipitation of aluminum and other leached species which is collected in the permeate product. In order to monitor the process conditions in the vessel, the vessel is equipped with temperature, level and pressure instruments. A pump that has a variable frequency drive transfers out the contents of the vessel. The contents can be transferred to HLP-VSL-T22 or to FRP-VSL-T01. The vessel is also equipped with a bottom drain which may be connected to UFP-VSL-T62A for evaporation considerations.

\subsubsection{Ultrafiltration Feed Preparation Vessel, UFP-VSL-T01A/B}

The ultrafiltration system is a batch system that requires a constant source of feed from UFP-VSL-T01A or B transferred to UFP-VSL-T02A. Therefore, for most ultrafiltration cycles consisting of producing $20 \mathrm{wt} \%$ solids, multiple batches of feed will be required to support ultrafiltration. To accomplish this the vessels UFP-VSL-T01A/B will likely operate on alternate phases, with one vessel receiving waste from HLP-VSL-T22 while the other vessel is either performing caustic leaching (if upfront leaching is required) or is in discharge mode. If caustic leaching is required, the vessel will undergo heating and digest modes before being placed in discharge mode. Once a vessel is in discharge mode, the vessel will feed small batches of waste to UFP-VSL-T02A as required. As the feed is transferred, the slurry is cooled to the filtering temperature as necessary. Cooling of the feed is performed using the single pass external cooling heat exchanger. The requirement for determining when to feed UFP-VSL-T02A is based on maintaining a constant volume of slurry in the vessel. The vessel will continue feed UFP-VSL-T02A 
until either the concentration step is complete or the vessel draws down to the minimum pump level. If the minimum pump level is reached before the concentration of solids is complete, the other preparation vessel will be switched to discharge mode. Transfer of waste is made using centrifugal pumps (UFPPMP-T41A/B). Besides transferring to UFP-VSL-T02A, these vessels may also make transfers between vessels (UFP-VSL-T01A and B).

Vessels UFP-VSL-T01A/B are equipped with prototypic pulse jet mixers (PJMs) that will be used to agitate the vessel contents and keep the solids suspended off the bottom. To accomplish this, the PJMs will essentially be running full time, whenever the vessel slurry level is above the minimum required mixing level. The PJMs will operate in different modes depending on the slurry level within the vessel. These modes of operation will prevent abnormal events such as overblow, flashing (e.g., boiling), and vessel overflow conditions from occurring.

Sampling in UFP-VSL-T01A/B occurs in either of two ways. First, the vessel is equipped with sample ports that allow for a representative sample from three locations within the vessel. Each sample port can be taken at different liquid levels. The second provision for sampling is off the recirculation loop. Sampling for this vessel may include analysis of efficiencies of waste blending and upfront caustic leaching.

The vessel is equipped with temperature, level, and pressure measurements to monitor process conditions. Density of the simulant is also available using the Coriolis flow/densitometer instrument.

Caustic Leach. Caustic leaching, whether "upfront" or "baseline," includes the steps of caustic addition, heating, digesting, and cooling the waste. If caustic leaching is required in the feed preparation vessel, the following steps occur: $19 \mathrm{M}$ sodium hydroxide is added as required; the caustic is added from three possible locations, in-line on the transfer from HLP-VSL-T22, added into FEP-VSL-T01 or added into UFP-VSL-T01A/B; the volume of transfer is controlled by flow totalizers on the reagent add lines or by the level of the vessel; if baseline leaching is required, the $19 \mathrm{M} \mathrm{NaOH}$ is added to the pump suction line of the two pumps in series (UFP-PMP-T42A/43A).

Next, the resulting caustic solution is heated to $100^{\circ} \mathrm{C}$. The time required to heat the fluid is scaled by the scaling factor, resulting in a heat-up time of 2 hours. The heating in tanks UFP-VSL-T01A/B and UFPVSL-T02A is achieved by direct steam injection. The temperature of the slurry is controlled by temperature instrumentation located at prototypic location in the vessel. During baseline leaching, an additional step is required. Supplemental heating exchangers are available in case maintaining temperature while keeping the water balance consistent with the full-scale plant proves to be difficult. If the external heat exchanger is used, the ultrafilters should be bypassed. If the external heat exchangers are not used, the ultrafilter loop should be drained to prevent non-Newtonian simulant from settling in the piping. The loop will be filled and flushed prototypic of the plant with three line volumes. During the last flush, the loop should be filled with inhibited water to keep the filters wet.

The next step of caustic leaching is to digest the slurry. Digestion involves maintaining the elevated temperature for eight hours. Temperature is controlled with temperature instrument inside the vessel. Steam condensate is monitored by level. 
Finally, the slurry is cooled back to the filtration temperature $\left(25^{\circ}-45^{\circ} \mathrm{C}\right.$ normally, $60^{\circ} \mathrm{C}$ maximum). The cooling function is accomplished differently depending on where caustic leaching is conducted. During upfront leaching cooling is performed using the single pass external cooling heat exchanger, whereas baseline leaching uses a cooling jacket on UFP-VSL-T02A. The time to cool is also different. Because the single pass heat exchanger cools the waste as it is transferred to UFP-VSL-T02A, the cool-down time is essentially instantaneous. However, cooling by jacket in UFP-VSL-T02A will be scaled time, resulting in a cooling time of 7 hours. During upfront leaching, once the vessel contents have been leached, the vessel is placed in discharge mode and is ready to transfer its contents to UFP-VSL-T02A. During baseline leaching, at this point in the cycle, the vessel would be ready to be placed in reconcentration mode.

If upfront leaching is not employed, the vessel contents still need to be cooled to the filter temperature using the in-line heat exchanger. During the discharge mode, the pumps will periodically transfer batches of feed to the UFPs (UFP-VSL-T02A), until the vessel reaches the minimum set point for pumping (i.e., minimum heel volume). Upon reaching the minimum set point for agitation, the PJMs are stopped.

\subsubsection{Ultrafiltration Feed Vessels, UFP-VSL-T02A}

After the appropriate scaled batch volume, indicated by UFP-VSL-T02A vessel level instrumentation or flow totalizers on the transfer line, has been transferred to UFP-VSL-T02A, the vessel is placed in concentration mode. Concentration involves recirculating the slurry through the ultrafilter loop and removing the liquid fraction (permeate) until the solids are concentrated to the target $20 \mathrm{wt} \%$ solids. The initial concentration of solids will be approximately $3-5 \mathrm{wt} \%$.

The operating temperature in UFP-VSL-T02A during ultrafiltration sequence is $25^{\circ}-45^{\circ} \mathrm{C}$, with a maximum operating temperature of $60^{\circ} \mathrm{C}$. During operation of the recirculation pumps, this temperature is maintained by the external cooling heat exchanger. The primary function of this heat exchanger is to remove heat generated in the form of pump and PJM energy to maintain a constant filter temperature.

The concentration mode is initiated by the following steps: Prior to starting the recirculation pumps, two centrifugal pumps in series, the permeate control valves are set to the closed position to eliminate the pressure drop across the ultrafilter membrane. The first ultrafiltration pump (UFP-PMP-T42A) is started and ramped up to the required pressure. Once the pre-enabled pressure is reached, the second pump (UFP-PMP-T43A) is started. This pump is equipped with a variable frequency drive that is used to set the recirculation flow rate and in turn the velocity through the filter modules. The flow rate of the pump is controlled by a flow meter. The flow rate of the slurry is controlled by adjusting the recirculation pump speed until the desired velocity $(12-15 \mathrm{ft} / \mathrm{sec})$ through the ultrafilter tube units is achieved. Once the velocity is reached, the tube side pressure is adjusted to provide sufficient loop pressure to achieve the required transmembrane pressure (TMP) of the last ultrafilter bundle in series. The pressure in the loop is controlled manually via the adjustable restriction valve on the ultrafilter loop return line. Next, a transmembrane pressure is effectively established over each ultrafiltration module by slowly opening the permeate control valves. Control of TMP is achieved by modulation of the permeate flow control valve. The transmembrane pressures are set to be identical for both ultrafilters. Typical transmembrane pressure will range from 30 to 70 psig. 
As the slurry is recirculated through the filter loop, the transmembrane pressure forces the liquid through the filters, creating permeate. Permeate is routed through pulse pots (UFP-PP-T01A, UFP-PP-T02A, UFP-PP-T03A) before being collected in the permeate vessels (UFP-VSL-T62A/B). Permeate from the separation process is collected primarily in UFP-VSL-T62B since UFP-VSL-T62A will largely be used as an evaporator/concentrator vessel. As the slurry is passed through the filters, the solids fraction is recirculated via the pumps back to the UFP vessel, where it is mixed and pumped back through the ultrafiltration loop. As the slurry is recirculated, a small portion of liquid is removed. This results in increased solids concentration. To account for the volume loss in the UFP vessels, fresh feed is periodically supplied from UFP-VSL-T01A/B. Concentration continues until approximately $20 \mathrm{wt} \%$ solids are generated in the ultrafiltration feed vessel. The vessel contents are ready for additional treatment.

\subsubsection{Leaching in UFP-VSL-T02A}

This section is described by exception to the "upfront" leaching section describe previously. After concentration of the solids to $20 \mathrm{wt} \%$, the following treatment sequence is initiated in UFP-VSL-T02A.

Following the concentration sequence, the solids may undergo further treatment steps. The purpose of treating the solids (as specified in Specification 12 of the contract) is to minimize the amount of immobilized HLW canisters produced. This is accomplished by washing or leaching out the soluble solids (e.g., aluminum, chrome) from the insoluble solids using inhibited water for solids washing and $1 \mathrm{M} \mathrm{NaMnO}_{4}$ for oxidative leaching. The soluble solids thus become part of the liquid fraction of the waste and are sent to LAW vitrification. The insoluble solids are sent to HLW vitrification. If not already leached (i.e., upfront leaching), the solids will be leached in UFP-VSL-T02A. Following leaching, the slurry is reconcentrated to $20 \mathrm{wt} \%$ using the same steps as in the concentration mode. The reconcentration step is required because during caustic leaching up to $50 \%$ of the solids are dissolved into the liquid fraction, thereby reducing the $\mathrm{wt} \%$ solids. Additional treatment steps, which include solids washing and oxidative leaching, are then conducted.

\subsubsection{Solids Prewash}

The solids washing step is complete when three times the initial batch volume of solids (concentrated to $20 \mathrm{wt} \%$ ) is collected in the permeate vessel. Therefore, if the batch volume of solids is 220 gal after reconcentration, the volume of wash permeate would be three times that, or 660 gal.

Solids washing uses the same methods as concentration mode, but instead of adding feed from UFP-VSL$\mathrm{T} 01 \mathrm{~A} / \mathrm{B}$, inhibited water is added incrementally to displace the sodium in the liquid. The focus of this pre-wash is to remove excess soluble components such as sodium through dilution and ultrafiltration. To accomplish this, inhibited water is added while the ultrafilter loop is running to dilute or remove the liquid from the solids. The wash permeate, which is considerably more dilute than the mother liquor permeate, will be collected in the permeate collection vessel (primarily UFP-VSL-T62A). The solids washing step is complete when three times the initial batch volume of solids (concentrated to $20 \mathrm{wt} \%$ ) is collected in the permeate vessel. Following solids washing is oxidative leaching. 


\subsubsection{Oxidative Leaching}

This step is similar to caustic leaching in that $1 \mathrm{M}$ sodium permanganate is added to the pump suction line and recirculated through the filter loop; once the reagent is added, the vessel is digested for 6 hours. Unlike caustic leaching, oxidative leaching does not require elevated temperature. After the appropriate digest time, the vessel contents undergo another solids washing identical to the first wash step.

\subsubsection{Solids Post-Washing}

The solids then undergo a post-wash using inhibited water. The inhibited water is added while the ultrafilter loop is running to dilute out the leach solution from the solids. As with the solids prewash step, the dilute wash permeate is collected in the permeate collection vessel (UFP-VSL-T62A) until the solids have been reconcentrated to $20 \mathrm{wt} \%$.

Due to solids washing and leaching steps, the maximum achievable solids concentration may be lower than the desired $20 \mathrm{wt} \%$ solids. The solids are then transferred to the HLW lag storage (HLP-VSL-T27A) where the final concentration will be determined by sample. After the solids have been transferred, the ultrafilters may need to be cleaned.

\subsubsection{Ultrafilter Cleaning Cycle}

Cleansing of the ultrafilters is accomplished using one of three methods. The first method consists of back-pulsing the filters from pulse-pots with permeate using high-pressure air as the motive force. The second option is to clean the ultrafilter loop with nitric acid, and the third option is to clean with caustic. Normal cleaning operations involves dilute caustic for rinsing/flushing coupled with back-pulsing. The following sections describe the operations required to achieve each method.

Cleaning with either acid or caustic will be carried out periodically. The current operating philosophy for cleaning the filters is to perform a cleaning cycle after the completion of the solid transfer sequence from UFP-VSL-T02A. The periodicity of back-pulsing is intermittent, but it may occur frequently as a normal operation of the ultrafilter loop (e.g., during a campaign).

\subsubsection{Ultrafilter Permeate Vessels, UFP-VSL-T62A/B}

Permeate is normally routed from the ultrafilter permeate vessels (UFP-VSL-T62A/B) to the Cs IX feed vessel (CXP-VSL-00001) in the cesium ion exchange process system (CXP) for further processing. However, the PEP is not integrated with ion exchange. The permeate collection in vessels UFP-VSLT62A/B will be stored, sampled, and disposed of as needed. The vessel contents can also be transferred via bottom drain to FEP-VSL-T01 for recycle.

Each vessel is equipped with a sample port, and grab samples can be taken. The vessels are equipped with a variable frequency drive mechanical agitator to mix the simulant. The energy of the mixing can be varied to characterize the simulant as homogenous or stratified depending on the test requirements. The vessels have temperature, level, and pressure monitoring. Vessel UFP-VSL-T62A does contain a means of concentrating the waste to minimize waste disposal; this is accomplished using a heating jacket to boil off excess water. The permeate vessels also have the capability to add caustic to test how the aluminum 
dissolved during caustic leaching behaves when left to sit and cool down. By adding additional caustic, the aluminum will be redissolved into solution.

\subsubsection{Permeate Back Pulsing}

During filtration, a very fine particulate layer of solids may form on the surface of the filter tube membrane. This thin solids layer results in a filter of much finer porosity than that of the porous metal tube, which allows for an increased degree of filtration to be achieved. As filtration continues, the solids layer may increase in thickness and some of the smaller particulates will penetrate into the filtration media and become stuck resulting in a reduced flow rate through the porous tube. To dislodge this solids layer from the membrane and to increase permeate flow rate, a backpressure pulse is passed through the clean side of the tubes. The frequency of the back-pulse will be determined from data collected from the demonstration testing. Additionally the back-pulse sequence, which is done automatically using the control system, can be manually over-ridden by an operator if required.

Back-pulsing consists of pressurizing the permeate lines to force permeate back through the filter from outside in. This action will remove particles lodged within the filter membrane and on the filter surface. Using Figure 2.3, the mechanisms necessary to achieve this method are defined. Normal operation requires that valves $\mathrm{A}$ and $\mathrm{C}$ to be open, while valves $\mathrm{B}, \mathrm{D}, \mathrm{E}$ and $\mathrm{F}$ are closed and that the pulse pots are constantly flooded. The permeate control valve is varied to supply the appropriate trans-membrane pressure. The following steps are initiated to back-pulse the ultrafilter:

- Stop permeate inlet and outlet by closing valves A and C.

- Open valve $\mathrm{D}$, and the process air inlet valve $\mathrm{F}$ until the pulse-pot is voided (to liquid level equivalent to nozzle N5). For the PEP system, the void volume is confirmed by level indication in the pulse-pots. For the plant system, because the level instrument is not available, voiding of the pulse-pot is based on a timed event (i.e., process air valve F is opened for a set amount of time). This method of controlling the permeate void volume may be demonstrated if desired in the PEP system.

- Close valve D and pressurize the pulse-pot to approximately 40 psig above the process inlet pressure.

- Close the process air inlet valve, F, and open the fast-acting valve B. Wait until the pressure equalizes.

- Open permeate outlet valve, $\mathrm{C}$, and begin controlling TMP by modulating the permeate control valve.

\subsubsection{Nitric Acid Wash}

Prior to initiating a nitric acid wash, the system configuration is assumed to be in normal operations, as defined above, and the solids transfer sequence is complete. The ultrafiltration feed vessel should be emptied, including the heel. The permeate lines should be drained and closed, and the ultrafilter loop should be drained and flushed with inhibited water to remove most of the solids. 


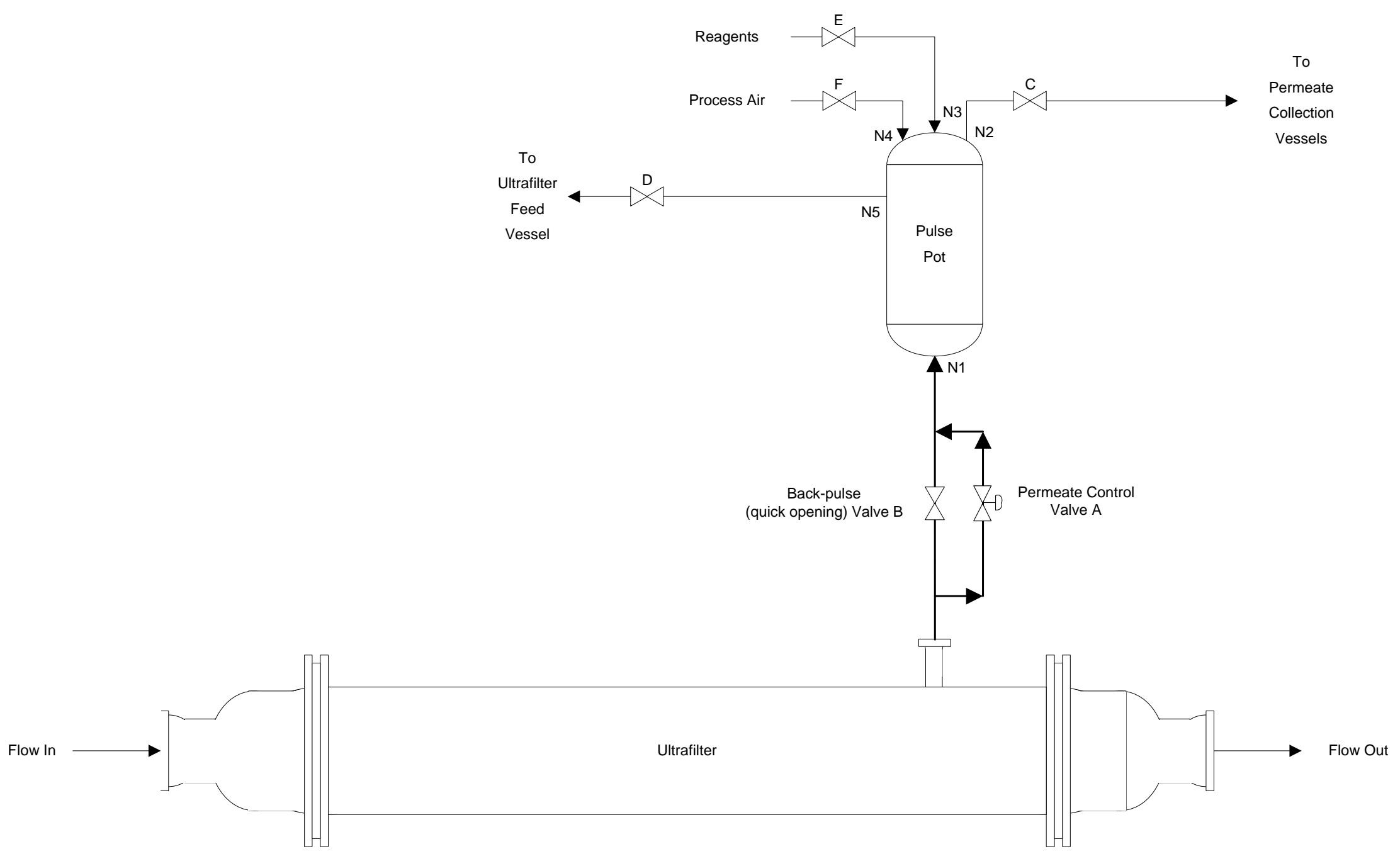

Note: Line length between the permeate nozzle and the pulse pot (N1) shall be less than 37.5 inches

Figure 2.3. Normal Operation of Ultrafilter Module and Permeate Process Diagram 
The ultrafiltration feed vessel is then filled with $2 \mathrm{M}$ nitric acid. The volume of $2 \mathrm{M}$ nitric acid is set using flow totalizers or level indication on the vessel. The recirculation pumps are initiated, and the nitric acid is recirculated for a set time (assumed 1.5 hours), at which point the nitric acid is drained from the vessel. As part of filter cleaning, acid may also be added directly to the pulse pots and back pulsed through the filters.

The filters will then be tested for effectiveness of the cleaning. If the cleaning provided undesirable results, another batch of nitric acid can be added as described above. After the cleaning is completed, confirmed by the testing of effectiveness the loop must be set back to its basic pH state. This is accomplished using inhibited water to recirculate through the loop. The loop is now ready for the next filtration demonstration.

\subsubsection{Caustic Wash}

If it is determined that the solids for a particular batch of feed are soluble in a highly caustic solution, caustic wash may be employed. The method for caustic wash follows the same sequencing as the nitric acid wash, using $2 \mathrm{M} \mathrm{NaOH}$ instead of nitric acid. 


\subsection{Hazard Analysis Methods}

Hazard analyses were performed for the 30\% design (based on Revision M PEP P\&IDs) (TKS 2007b), 90\% design (based on Revision 3 PEP P\&IDs) (TKS 2007c), and on the final 100\% design (based on Revision 7 PEP P\&IDs)(TKS 2007X).

The hazard analysis for the $30 \%$ PEP was divided into three parts. The first session was used to complete the hazard identification for the entire PEP project (both the skid system and the PDL-W). The second session supported the design of the skid-mounted units at 30\% design and evaluated the potential hazards of operating the skid-mounted systems as designed. The results from the sessions were integrated into the $60 \%$ design review to verify that the design was safe. The third session was conducted to evaluate the hazards of integrating the skid-mounted units into the existing PNNL facility, PDL-W.

At the $90 \%$ PEP design hazard analysis, the $30 \%$ results were reviewed and modified for applicability to the updated design package. Any changes that were required and any additional hazardous conditions identified were added.

At the final 100\% PEP design hazard analysis, the $90 \%$ results were reviewed and modified for applicability to the updated design package. Any changes that were required and any additional hazardous conditions identified were added. All open recommendations from the previous sessions were reviewed/resolved prior to the final session. In addition, an evaluation was performed to assign the final set of safeguards (credited safeguards) to the hazardous conditions.

A description of the methods used to perform all of these activities is provided in the following sections. Results of the hazard analysis activities are contained in Section 4.

\subsection{Hazard Identification}

For the hazard identification activity, a hazard is defined as an energy source or harmful material. All hazards and energy sources applicable to the facility were identified on a Hazard Identification Checklist. The hazard analysis team systematically reviewed the checklist for application to the PEP, and those hazards that were applicable were checked. Applicable hazards are considered those that are introduced by the project itself and those that are located close by that could affect the project. The final checklist is used during the hazard evaluation sessions to help in brainstorming the hazardous conditions that could potentially occur due to the presence of the hazards.

When one is completing a hazard identification checklist, the material-at-risk (MAR) is identified. This information addresses hazardous material that exists in the PEP process or facility and its quantity and potency. The information is recorded to help with the hazard evaluation sessions.

\subsection{Hazard Evaluation}

The hazard evaluation techniques were selected from Guidelines for Hazard Evaluation Procedures (AIChE 1992). For the PEP skid processes, a hazard and operability study (HAZOP) was used to identify 
potentially hazardous conditions and estimate their potential harm. For the integration of the PEP into the existing PDL-W facility, a preliminary hazard analysis (PHA) was used. A hazardous condition is defined to be a condition or combination of conditions that result in uncontrolled release of MAR, personnel injury, environmental insult, or facility damage.

A decomposition of the system into system nodes is used for the HAZOP process. System nodes are made up of system components where the system's physical parameters are similar, allowing easier evaluation of the effects of parameter changes. A decomposition of facility missions into activity nodes is made to support the PHA process. Activity nodes are made up of the variety of activities that can occur at specified locations of the facility. The decomposition used during the hazard evaluation sessions is shown in Appendix B based on the configuration of the 100\% design.

The HAZOP and PHA sessions are systematic brainstorming processes involving a multidisciplinary team of knowledgeable individuals. Results are captured on worksheets, which are described in Section 3.2.1. Because these assessments are to be qualitative in nature, the expertise and experience of the team is of primary importance in establishing the credibility of the analysis. Facility personnel representing operations, engineering, facility, and industrial safety were invited to attend the meetings and represent the desired core team. A wide-ranging set of significant hazardous conditions was formulated. The sessions started with development of preparatory information: 1) hazard and energy source identification, 2) definition of the MAR, and 3) decomposition of process into nodes. Based on this information, brainstorming of hazardous conditions follows. To assist the brainstorming in the HAZOP sessions, a process deviation guide is used. Each process parameter and the possible deviations are evaluated at each of the system nodes to help identify the potential release conditions. The HAZOP deviation guide is shown in Appendix C. During the PHA, all tasks related to each activity, as well as the failure of associated personnel equipment and systems, are considered while identifying potential release conditions.

From these activities, a wide-ranging set of hazardous conditions that could lead to release of hazardous materials from the PEP process was formulated. Events were identified during the course of the HAZOP that were considered operational upsets because they did not result in a risk to facility workers. These events were captured in the hazard analysis results for completeness, however, not all information was populated because there is no risk associated with these events.

\subsubsection{Hazards Analysis Worksheets}

The worksheets that were used to capture the information resulting from the hazard analysis sessions contained a series of columns where information was entered for each identified hazardous condition.

- Scenario number-The identifier is a unique code for each hazardous condition. It contains an indication of the project (PEP), the percent design of the session that the item is postulated $(30,90, \mathrm{FN})$, and node/activity related to the entry.

- Node-The decomposition node assessed for hazardous conditions.

- Hazardous condition-A brief description of the event that is postulated.

- Cause-A brief description of the cause leading to the hazardous condition, generally an identification of the initiating event. 
- Likelihood-Categorization used in estimating the frequency of the hazardous condition (Table 3.1).

- Consequence-Categorization used in estimating the consequence of the hazardous condition (Table 3.2).

- Environmental (E)-Categorization used in estimating the environmental consequence of the hazardous condition (Table 3.3).

- Financial (\$)-Categorization used in estimating the financial consequence of the hazardous condition (Table 3.4).

- Risk score-Categorization used in estimating the risk (based on the likelihood and consequence assigned) associated with the hazardous condition (Table 3.5).

- Potential safeguards-Engineering features or administrative controls that are currently planned to be designed/implemented as preventive or mitigative features. NOTE: No safeguards are identified for those items that are considered to have no risk.

- Credited safeguards - These are the safeguards identified at the final hazard analysis session that will be credited for minimizing the risk associated with the hazardous condition. These are usually, but not always, a subset of the potential safeguard list.

- Recommendations-Recommendations by the analysis team for additional safeguards that should be considered to prevent or mitigate the potential risk. This column was used during the $30 \%$ and $90 \%$, but was cleared at the final $100 \%$ design session due to all recommendations being reviewed/resolved.

- Comments/assumptions-Notes about assumptions used or ideas that were important to the event being identified.

\subsubsection{Likelihood Category Definitions}

The likelihood of each hazardous condition occurring was estimated by assigning one of the categories defined in Table 3.1.

Table 3.1. Likelihood Category Definitions

\begin{tabular}{||c|l|l||}
\hline Score & Definition of Likelihood & \multicolumn{1}{c||}{ Description } \\
\hline \hline 1 & Regular or periodic & > one event per year \\
\hline 0 & Occasionally & $\leq$ one 1 event per year > one event every 10 years \\
\hline-1 & Infrequently & $\leq$ one 1 event every 10 years > one event every 100 years \\
\hline-2 & Rarely & $\leq$ one event every 100 years > one event every 1,000 years \\
\hline-3 & Extremely rare & $\leq$ one event every 1,000 years > one event every 10,000 years \\
\hline-4 & Negligible & $\leq$ one event every 10,000 years \\
\hline
\end{tabular}




\subsubsection{Consequence Category Definitions}

The health and safety consequence of each hazardous condition was estimated by assigning one of the categories defined in Table 3.2.

Table 3.2. Health and Safety Consequence

\begin{tabular}{|c|l|l|l|l||}
\hline Score & $\begin{array}{l}\text { Qualitative } \\
\text { Descriptor }\end{array}$ & \multicolumn{1}{|c|}{ One Person Onsite } & Two Persons Onsite & $\begin{array}{l}\text { Impact Site-Wide, Affects } \\
\text { Three or More Persons }\end{array}$ \\
\hline \hline 6 & Catastrophic & -- & -- & Multiple fatalities \\
\hline 5 & Extreme & -- & Multiple fatalities & $\begin{array}{l}\text { Immediate impairment and } \\
\text { permanent health effects }\end{array}$ \\
\hline 4 & Severe & Fatality & $\begin{array}{l}\text { Multiple injuries with } \\
\text { immediate impairment and } \\
\text { permanent health effects }\end{array}$ & $\begin{array}{l}\text { Lost time accidents/injuries } \\
\text { requiring hospitalization }\end{array}$ \\
\hline 3 & Moderate & $\begin{array}{l}\text { Immediate impairment and } \\
\text { permanent health effects }\end{array}$ & $\begin{array}{l}\text { Lost time accidents/injuries } \\
\text { requiring hospitalization }\end{array}$ & $\begin{array}{l}\text { Multiple medical treatment } \\
\text { injuries }\end{array}$ \\
\hline 2 & Low & $\begin{array}{l}\text { Lost time accident/injury } \\
\text { requiring hospitalization }\end{array}$ & $\begin{array}{l}\text { Multiple medical treatment } \\
\text { injuries }\end{array}$ & $\begin{array}{l}\text { Multiple minor injuries, } \\
\text { first-aid cases and/or up to }\end{array}$ \\
\hline 1 & Minimal & Medical treatment injury & Minor injury/first-aid case & None \\
\hline 0 & Negligible & Minor injury/first-aid case & None & None \\
\hline \hline
\end{tabular}

In addition to consequences to the health and safety of personnel, potential financial impacts were also estimated, as shown in Table 3.3. The health and safety of the workers was the primary focus of the analysis. If the safety and health consequence score was obviously greater than the expected financial consequence score, only the health and safety score was ranked and recorded. An "SD" was then entered into the financial column to represent the assessment that the safety and health consequences would dominate the risk result. If it was not clear, both consequences were assessed and the risk value assigned based on the higher value.

Table 3.3. Financial Consequence

\begin{tabular}{|c|l||}
\hline \hline Score & \multicolumn{1}{|c||}{ Definition $^{(\mathbf{a})}$} \\
\hline \hline 6 & More than $\$ 1,000,000$ of production loss/facility damage \\
\hline 5 & $\$ 500,000-\$ 1,000,000$ of production loss/facility damage \\
\hline 4 & $\$ 250,000-\$ 500,000$ of production loss/facility damage \\
\hline 3 & $\$ 100,000-\$ 250,000$ of production loss/facility damage \\
\hline 2 & $\$ 50,000-\$ 100,000$ of production loss/facility damage \\
\hline 1 & $\$ 25,000-\$ 50,000$ of production loss/facility damage \\
\hline 0 & Up to $\$ 25,000$ of production loss /facility damage \\
\hline $\begin{array}{l}\text { (a) Financial consequences assessed in the PEP hazard analysis did not } \\
\text { include cost of lost test time. }\end{array}$ \\
\hline
\end{tabular}




\subsubsection{Environmental Category}

To track the potentially impacts of the potential environmental assault associated with the operation of the PEP, an environmental category was assigned to each postulated hazardous condition. The environmental categories are shown in Table 3.4. These categories are used to verify that safeguards are in place, where needed, to protect the environment as well as the health and safety of personnel.

Table 3.4. Environmental Consequence

\begin{tabular}{||l|l||}
\hline $\begin{array}{c}\text { Environmental } \\
\text { Consequence Category }\end{array}$ & \multicolumn{1}{c|}{ Definition } \\
\hline \hline E0 & No reporting required \\
\hline E1 & Minor remedial actions, but reporting required \\
\hline E2 & Major remedial actions, reporting required \\
\hline
\end{tabular}

\subsubsection{Risk Score}

Once the hazardous conditions are postulated and frequency and consequence categories assigned, risk scored are applied. The Risk Score is the sum of the likelihood and consequence scores and provides a measure of the significance of the event, as presented in Table 3.5.

Table 3.5. Risk Score

\begin{tabular}{|c|c||}
\hline Score & Score Description \\
\hline \hline 0 or less & Minimal Hazard \\
\hline 1 & Minor Hazard \\
\hline 2 & Moderate Hazard \\
\hline 3 & Serious Hazard \\
\hline 4 & Severe Hazard \\
\hline 5 or above & Critical Hazard \\
\hline
\end{tabular}

The degree of safeguard needed to protect safety must be commensurate with the risk; therefore, the risk scores are used to determine which events should be considered for additional safeguards as the design progresses. The goal is to assign a level of safeguards that will lower the risk score to as close to zero as practical (controlled risk $=$ safeguard score + likelihood score + consequences score). An example of the safeguard score that is applied is shown in Table 3.6. The safeguard score in Table 3.6 was used as guidance to select the types of controls to be considered to manage risk. However, passive design features (e.g., vessel design) were also considered as controls to reduce risk. In the case of industrial hazards (e.g. electrocution), standard industrial safety practice was generally considered adequate to manage risk. 
Table 3.6. Safeguard Score

\begin{tabular}{|c|l||}
\hline Score & \multicolumn{1}{c|}{ Score Description } \\
\hline \hline 0 & No safeguard of any source \\
\hline 1 & Operator round or procedural check \\
\hline 2 & Operator round or procedural check plus hardware alarm \\
\hline 4 & $\begin{array}{l}\text { Operator round or procedural check plus two } \\
\text { independent alarms/interlocks }\end{array}$ \\
\hline 6 & $\begin{array}{l}\text { Operator round or procedural check plus three } \\
\text { independent alarms/interlocks }\end{array}$ \\
\hline
\end{tabular}

The risk score presented in the hazard analysis worksheet is assigned based on the highest consequence score applied to a postulated hazardous condition. If the safety and health consequence is dominating then the risk is applied using the health and safety consequence score; if financial is higher then that score is used. This method verifies that the greatest level of safeguard is applied to each condition. 


\subsection{Hazard Analysis Results}

As discussed in Section 3, the hazard analysis activities were conducted at the 30\% design (based on the Revision M P\&IDs) (TKS 2007b.), the 90\% design (based on the Revision 3 P\&IDs) (TKS 2007c.), and the final 100\% design (based on Revision 7 PEP P\&IDs)(TKS 2007X). The 30\% design hazard analysis effort was divided into three parts: hazard identification for the entire PEP (both the skid system and the PDL-W); HAZOP of the skid-mounted units at 30\% design, and; PHA of the integration of the skidmounted units into the existing PNNL facility, PDL-W. The $90 \%$ review consisted of a gap analysis, which identified changes made to the design since the $30 \%$ review. Then the $90 \%$ review evaluated these design changes. The final $100 \%$ review consisted of a gap analysis, which identified changes made to the design since the $90 \%$ hazard analysis session and the closure of the recommendations. Then the 100\% hazard analysis evaluated these design changes and finalized the safeguard selection.

\subsection{Hazard Analysis Sessions}

The hazard identification session was held on May 29, 2007, in Richland, Washington. This meeting was combined with the system nodalization to support the HAZOP and included:

- Discussion of the PEP design at 30\%.

- Completion of the hazard identification checklist for hazards present in the PEP skid system and in the existing PDL-W facility.

- Identification of the MAR expected to be in the PEP system and at/nearby the PDL-W facility.

- Definition of the PEP skid system nodalization.

On June 4, 5, and 6, 2007, HAZOP sessions were held in Carlsbad, New Mexico, to postulate the potential hazardous conditions associated with operation of the skid-mounted PEP system. The following activities occurred during these HAZOP meetings.

- Discussion of the system design at $30 \%$.

- Review/update of the hazard identification check list and MAR list.

- Review of the nodes defined.

- Discussion of assumptions to be followed for performing the HAZOP.

- Completion of the worksheets using PHA protocol.

On June 19, 20, and 21, 2007, PHA sessions were held in Richland, Washington, to postulate the potential hazardous conditions associated with the integration of the skid-mounted PEP system into the existing PDL-W facility. The following activities occurred during the PHA meetings.

- Discussion of the PDL-W facility and how the PEP system will be housed.

- Review/update of the hazard identification check list and MAR list.

- Definition of the nodes based on the activities that will required at PDL-W once the PEP is integrated. 
- Discussion of assumptions to be followed for performing the PHA.

- Completion of the worksheets using PHA protocol.

On September 19, 2007, a gap analysis session was held in Richland, Washington, to review the design and identify design changes between the $30 \%$ and the $90 \%$ designs. These changes were subsequently evaluated on October 11, 2007, in a hazard evaluation session that was held in Richland. The following activities occurred during the meetings.

- Review the $30 \%$ hazard identification checklist results to identify applicability to the new design and make modifications as required.

- Review the $30 \%$ hazard evaluation results to identify applicability to the new design and make modifications as required.

- Evaluate, using the HAZOP methodology, any additional design information that was not available at the $30 \%$ design stage.

On March 10, 2008, a gap analysis was held in Richland, Washington, to review the design and identify design changes between the $30 \%$ and the $90 \%$ designs.

On April 1, 2008, a session was held in Richland, Washington, to review the operations procedures and identify any areas that may require any additional PHA review.

On April 15 and 17, the final gap was evaluated. The following activities occurred during the meetings.

- Review the $90 \%$ hazard identification checklist results to identify applicability to the new design and make modifications as required.

- Review the $90 \%$ hazard evaluation results to identify applicability to the new design and make modifications as required.

- Evaluate, using the HAZOP methodology, the gaps that were identified from the gap analysis between the $90 \%$ design and the final 100\% design.

- Evaluate, using the PHA methodology, the gaps in new information from the procedure review that were not available during the $90 \%$ design session.

- Once all hazardous condition postulation was complete, each condition was assessed again, and the credited safeguards were identified. This list is considered the final safeguard list for the PEP (see Section 5.0, Table 5.1).

The lists of attendees that supported each of the above meetings are included in Appendix A. The results of these activities are discussed in the following sections.

\subsection{Hazard Identification and MAR Definition}

The completion of the hazard identification checklist was performed as described in Section 3.1. The resulting checklists are included in Appendix D. During the identification and evaluation of process potential, MARs were discussed that could potentially be a hazard during an event. These MARs are defined as follows: 
- Simulant. The system receives simulant feed for demonstration of solids treatment and filtration. The feed is processed through the entire system. Simulant feed can be received in the following vessels: HLP-VSL-T22, FEP-VSL-T01 and FRP-VSL-T01.

- Nitric acid. $2 \mathrm{M}$ nitric acid for cleaning of the ultrafilters and $\mathrm{pH}$ adjustments in the condensate collection vessel ventilation system.

- Oxalic acid. An alternative chemical cleaning reagent, oxalic acid, may be used for acid cleaning of the filters if required. This alternative reagent would replace the use of nitric acid and will be added into the existing acid chemical storage tank (NAR-VSL-T01) if needed.

- Sodium hydroxide. $19 \mathrm{M} \mathrm{NaOH}$ addition for caustic leaching. $2 \mathrm{M} \mathrm{NaOH}$ for cleaning of the ultrafilters and $\mathrm{pH}$ adjustments in the condensate collection vessel ventilation system.

- Sodium permanganate. $1 \mathrm{M} \mathrm{NaMnO}_{4}$ for oxidative leaching.

- Inhibited water. Treated water $(0.01 \mathrm{M} \mathrm{NaOH})$ used for solids washing or dilutions.

\subsection{Nodal Decomposition}

To support the hazard evaluation process the PEP skid system was decomposed into system nodes and the PDL-W mission was decomposed into activity nodes, as discussed in Section 3.2. The decomposition used during the hazard evaluation sessions are shown in Appendix B. The nodes that were used during the $30 \%$ design evaluation are shown in B.2, and the nodes for the $90 \%$ design are in B.3.

\subsection{Assumptions}

Assumptions and ground rules were defined during the analysis process to support decisions made during the hazard analysis activities. The assumptions were reviewed during the $90 \%$ hazard analysis activities and the updated significant assumptions are listed in Table 4.1.

\subsection{Results}

The hazard analysis worksheets are included in Appendix E and show the results of the hazard analysis activities that were conducted up to and including the $100 \%$ design. Assumptions that were necessary for completing the hazard analysis activities are included in Table 4.1. 
Table 4.1. Hazard Analysis Assumptions/Ground Rules

\begin{tabular}{|c|c|}
\hline \# & Assumption/Ground Rule \\
\hline 1 & $\begin{array}{l}\text { During operation of the facility, there will only be one operator in the direct vicinity of a hazard at } \\
\text { one time who will be exposed to the direct effects }\end{array}$ \\
\hline 2 & $\begin{array}{l}\text { The health and safety of the workers was the primary focus of the analysis. If the safety and health } \\
\text { consequence score was considered greater than the expected financial consequence score, only the } \\
\text { health and safety score was ranked and recorded. Safeguards were applied based on this value. An } \\
\text { "SD" was then entered into the financial column to represent safety and health dominated. }\end{array}$ \\
\hline 3 & $\begin{array}{l}\text { When determining the financial consequence score, the monetary contribution of process "down- } \\
\text { time" was not included in the associated costs. The consequence score includes the immediate } \\
\text { financial impact of any damage and the associated repair. }\end{array}$ \\
\hline 4 & $\begin{array}{l}\text { If the consequences were considered negligible for both the safety and health and the financial } \\
\text { scores, the event was considered operational; upset only. No likelihood or risk was assigned. }\end{array}$ \\
\hline 5 & $\begin{array}{l}\text { In the steam system, the boiler design was considered a black box because this is yet to be provided } \\
\text { by the vendor. }\end{array}$ \\
\hline 6 & $\begin{array}{l}\text { No mapping between nodes was included in the worksheet. If an event was already discussed in a } \\
\text { previous node, it was not reentered in another (items discussed in a process system node was not } \\
\text { repeated when discussed in a utility node.) }\end{array}$ \\
\hline 7 & $\begin{array}{l}\text { Quick fitting disconnects on Node } 9 \text { have redundant closure so no potential discharges were } \\
\text { postulated. }\end{array}$ \\
\hline 8 & The deadhead of the ultrafiltration pumps is 400 psi. \\
\hline 9 & $\begin{array}{l}\text { There are no physical connections between the chemical addition tanks that supply the additional } \\
\text { acid and base. }\end{array}$ \\
\hline 10 & $\begin{array}{l}\text { Chemicals will be received in liquid form (no dry mixing required). However, the concentrations of } \\
\text { these chemicals as received are currently not identified. }\end{array}$ \\
\hline 11 & The acid tank is vented into the process ventilation system. \\
\hline 12 & flows on the chemical addition tanks are designed to go over to a small catch basin. \\
\hline 13 & $\begin{array}{l}\text { Analytical capabilities within PDL are yet to be determined. The activities that will occur in the lab } \\
\text { will be performed per PNNL procedures as defined by SBMS and IOPS. }\end{array}$ \\
\hline 14 & $\begin{array}{l}\text { The hazard analysis covers operation and maintenance activities after construction is complete. } \\
\text { Construction includes the hydro testing of the system. The scope of this HA starts at acceptance } \\
\text { testing of the system by PNNL. }\end{array}$ \\
\hline
\end{tabular}


Table 4.1 (contd)

\begin{tabular}{|c|l||}
\hline \hline$\#$ & \multicolumn{1}{|c|}{ Assumption/Ground Rule } \\
\hline 15 & $\begin{array}{l}\text { The shake-down test plan (Node E1) will adequately address the safety hazards associated with the } \\
\text { plan. The plan should be reviewed when developed for consistency with the developed hazard } \\
\text { analysis. }\end{array}$ \\
\hline 16 & $\begin{array}{l}\text { The spill response plan will address the recovery actions from a major spill. The plan should be } \\
\text { reviewed, when developed, for its consistency with the developed hazards. }\end{array}$ \\
\hline 17 & $\begin{array}{l}\text { Major recovery actions will require analysis as part of a recovery plan and JHA. These are not } \\
\text { covered in detail in this HA. }\end{array}$ \\
\hline 18 & $\begin{array}{l}\text { During equipment repair, a crane will be used to remove and move ultrafilters to the north end of the } \\
\text { building. }\end{array}$ \\
\hline 19 & The ultrafilters are not now able to be isolated individually from the system during repair activities. \\
\hline 20 & External events will be analyzed at the next iteration of the hazard analysis. \\
\hline 21 & $\begin{array}{l}\text { Information on the steam trap was not available at this time; it will be analyzed in detail when } \\
\text { available. }\end{array}$ \\
\hline 22 & $\begin{array}{l}\text { Facility secondary containment provides protection against environmental release in the case of spills } \\
\text { from process vessels. It is considered to be present when postulating environmental impacts in the } \\
\text { hazard analysis. }\end{array}$ \\
\hline 23 & $\begin{array}{l}\text { Titration testing performed by F\&O not covered by this HA. Those activities are covered by PNNL } \\
\text { SBMS. }\end{array}$ \\
\hline 24 & $\begin{array}{l}\text { Transfer of samples to the testing facility is covered by standard PNNL procedures. Not analyzed } \\
\text { during the HA. }\end{array}$ \\
\hline 25 & Sample cabinet can hold up to 50 liters of samples. \\
\hline 26 & $\begin{array}{l}\text { Packaging of samples by FSR within PDL-W will be covered by their standard procedures and are } \\
\text { not specifically analyzed in this HA. }\end{array}$ \\
\hline 27 & Evaluation of materials of construction was considered to be outside the scope of the hazard analysis. \\
\hline
\end{tabular}




\subsection{Safeguards}

To support the development of the PEP design and identify the final set of credited safeguards, recommendations were generated during the hazard analysis activities. These recommendations fall into three categories:

- Develop additional information on the design, operation, or maintenance of PEP to support completion of the hazard analysis.

- Perform engineering analyses to confirm design assumptions or bases.

- Provide additional safeguards not identified in the 30\% design package or intended operating scheme.

Appendix F contains an historical record of the hazard analysis-related recommendations generated during the design development process. It includes the types of conditions addressed by the recommendations and associated nodes from the hazard analysis results (Appendix E). The "\%" column specifies at which hazard analysis session the recommendation was made and provides a unique identifier. All of the recommendations have been reviewed by the Project and resolved.

During the final hazard analysis (100\% design), the credited safeguards were selected for each hazardous condition. The safeguards applied to each hazardous condition are included in the results in Appendix E. Table 5.1 summarizes the credited safeguards and identifies the highest condition risk level to which it is applied.

The degree of safeguard selected is commensurate with the level of risk for the specific condition; therefore, the risk score associated with each condition was used to determine the level of safeguards needing to be identified. The goal is to assign a level of safeguards that will lower the risk score to as close to zero as practical (controlled risk $=$ safeguard score + likelihood score + consequences score). The criteria identified in Section 3.2.5 were used as guidelines for selecting safeguards. In addition, the following rules where also used to aid decisions on hierarchy of safeguard preference when multiple safeguards where available:

- Preventive safeguards over mitigative safeguards

- Passive safeguards over active safeguards

- Engineering safeguards over administrative safeguards

- Safeguards with the highest reliability

- Safeguards closest to the hazard.

In the case of industrial hazards (e.g., electrocution), the standard industrial safety practice was generally considered adequate to manage risk. Conditions that are already at a risk of 0 or lower were not assessed for safeguards. 
Table 5.1. PEP Credited Safeguards

\begin{tabular}{|c|c|c|}
\hline $\begin{array}{l}\text { Safeguards Selected for the PEP at Final } \\
\text { Hazard Analysis }\end{array}$ & Type(s) of Hazardous Condition Addressed & $\begin{array}{l}\text { Highest } \\
\text { Risk } \\
\text { Score } \\
\text { Applied }\end{array}$ \\
\hline 90-day storage pad secondary containment & $\begin{array}{l}\text { Personnel injury due to exposure to process } \\
\text { waste }\end{array}$ & 3 \\
\hline Accumulator designed to code & Personnel injury due to debris & 1 \\
\hline Administrative controls for stop work & Personnel heat stress & 3 \\
\hline Bollards around gas connection & Natural gas fire outside facility & 2 \\
\hline $\begin{array}{l}\text { Building HVAC with temperature } \\
\text { monitoring }\end{array}$ & Personnel heat stress & 3 \\
\hline Chemical tank level detection and alarm & Spill of chemicals & 4 \\
\hline Design boiler to code & Large steam release & 3 \\
\hline Design of chemical tanks & Spill of chemicals & 1 \\
\hline Design of feed preparation tanks to code & Spill of tank contents & 1 \\
\hline Design of filter assembly & $\begin{array}{l}\text { Spill of simulant, sodium hydroxide, sodium } \\
\text { permanganate, nitric acid, or inhibited water }\end{array}$ & 1 \\
\hline Design of permeate receipt tanks to code & Tanks failure and spill & 1 \\
\hline $\begin{array}{l}\text { Design of platform, stairs (grating, railings, } \\
\text { toe boards) }\end{array}$ & Personnel injury due to fall, impact & 5 \\
\hline Design of pulse pot to code & Pressurized leak of permeate & 3 \\
\hline Design of receipt tanks to code & $\begin{array}{l}\text { Spills of simulant, sodium hydroxide, water, } \\
\text { materials from other tanks }\end{array}$ & 1 \\
\hline Design of sample cabinet (Conex) & Release of material outside facility & 0 \\
\hline Design of sample container & $\begin{array}{l}\text { Release of material outside facility; exposure to } \\
\text { hot chemicals }\end{array}$ & 3 \\
\hline Design of Slurry Concentrate tank to code & Tank failure and spill & 1 \\
\hline Design of steam jacket & & 2 \\
\hline Design of steam shell to code & Large steam release & 2 \\
\hline Design of steam system to code & Small steam release; large steam release & 6 \\
\hline Design of ultrafiltration feed tank to code & Tank failure and spill & 1 \\
\hline DOT containers for Nitric & Personnel injury due to exposure of fumes & 4 \\
\hline Facility secondary containment & $\begin{array}{l}\text { Spills of simulant, sodium hydroxide, water, } \\
\text { materials from other tanks }\end{array}$ & 5 \\
\hline Feed Preparation tank level detection & $\begin{array}{l}\text { Spills of simulant, sodium hydroxide, water, } \\
\text { materials from other tanks; carryover of material } \\
\text { into ventilation system; injury due to debris }\end{array}$ & 4 \\
\hline $\begin{array}{l}\text { Feed Preparation tank level detection and } \\
\text { alarms }\end{array}$ & Spill of tank contents; out of spec batch & 3 \\
\hline Fire alarm & Facility fire; personnel injury due to fire & 3 \\
\hline $\begin{array}{l}\text { Hoisting, Rigging, Forklifts, and Aerial } \\
\text { Lifts Requirements }\end{array}$ & $\begin{array}{l}\text { Personnel injury due to exposure to process } \\
\text { waste, impact; damage to facility structure }\end{array}$ & 4 \\
\hline
\end{tabular}


Table 5.1 (Contd)

\begin{tabular}{|c|c|c|}
\hline $\begin{array}{l}\text { Safeguards Selected for the PEP at Final } \\
\text { Hazard Analysis }\end{array}$ & Type(s) of Hazardous Condition Addressed & $\begin{array}{l}\text { Highest } \\
\text { Risk } \\
\text { Score } \\
\text { Applied }\end{array}$ \\
\hline $\begin{array}{l}\begin{array}{l}\text { Incompatible fitting } \\
\text { chemical types }\end{array} \\
\end{array}$ & $\begin{array}{l}\text { Personnel injury due to exposure to chemicals; } \\
\text { spill of material inside facility }\end{array}$ & 5 \\
\hline Insulation on piping & Personnel injury due to burn & 2 \\
\hline Insulation on piping and tank sides & Personnel injury due to burn & 4 \\
\hline JHA and work plan & $\begin{array}{l}\text { Personnel injury due to impact; electrical shock; } \\
\text { steam burn; missile impact, or exposure to } \\
\text { process material; equipment damage }\end{array}$ & 5 \\
\hline Lighting (facility emergency) & Personnel injury due to tripping, falling & 5 \\
\hline Lighting (normal and facility emergency) & Personnel injury due to fall, impact & 5 \\
\hline Machine guards on agitators and pumps & Personnel injury due to rotating equipment & 3 \\
\hline Natural gas system is designed to code & Natural gas fire outside facility & 2 \\
\hline Operating procedures & $\begin{array}{l}\text { Spills of simulant, sodium hydroxide, sodium } \\
\text { permanganate, nitric acid, inhibited water, or } \\
\text { materials from other tanks; out of spec batch. } \\
\text { Contaminate chilled water/steam; release of } \\
\text { material from Hx heated loop; collapse of steam } \\
\text { shell; small-steam release; large steam release; } \\
\text { hot water release; aerosol release from Nitric } \\
\text { Acid tank; over flow of tank into ventilation } \\
\text { system; damage equipment; personnel injury due } \\
\text { to burn, release of process materials, exposure to } \\
\text { chemicals; exposure to hot chemicals; small } \\
\text { release of material }\end{array}$ & 6 \\
\hline Operator rounds & Carryover of material into ventilation system & 3 \\
\hline Operator rounds (visual identification) & Spray of $19 \mathrm{M}$ caustic & 1 \\
\hline Permeate evaporation tank level detection & Damage equipment & 5 \\
\hline $\begin{array}{l}\text { Permeate evaporation tank temperature } \\
\text { indication }\end{array}$ & Damage equipment & 5 \\
\hline Permeate receipt tank level detection & $\begin{array}{l}\text { Spill of permeate, concentrated slurry, simulant, } \\
\text { sodium hydroxide, sodium permanganate, nitric } \\
\text { acid, or inhibited water }\end{array}$ & 5 \\
\hline $\begin{array}{l}\text { Permeate receipt tank level detection and } \\
\text { alarm }\end{array}$ & $\begin{array}{l}\text { Overflow of tanks into ventilation system; spill } \\
\text { of permeate, concentrated slurry, simulant, } \\
\text { sodium hydroxide, sodium permanganate, nitric } \\
\text { acid, or inhibited water }\end{array}$ & 3 \\
\hline PJM Level Detector & $\begin{array}{l}\text { Carryover of material into ventilation system; } \\
\text { injury due to debris }\end{array}$ & 4 \\
\hline PJM Pressure Detector & $\begin{array}{l}\text { Carryover of material into ventilation system; } \\
\text { injury due to debris }\end{array}$ & 4 \\
\hline
\end{tabular}


Table 5.1 (Contd)

\begin{tabular}{|c|c|c|}
\hline $\begin{array}{c}\text { Safeguards Selected for the PEP at Final } \\
\text { Hazard Analysis }\end{array}$ & Type(s) of Hazardous Condition Addressed & $\begin{array}{l}\text { Highest } \\
\text { Risk } \\
\text { Score } \\
\text { Applied } \\
\end{array}$ \\
\hline PJM Rack Air Supply Valve & $\begin{array}{l}\text { Carryover of material into ventilation system; } \\
\text { injury due to debris }\end{array}$ & 4 \\
\hline PLC & $\begin{array}{l}\text { Carryover of material into ventilation system; } \\
\text { injury due to debris }\end{array}$ & 4 \\
\hline PPE & $\begin{array}{l}\text { Spills of simulant, sodium hydroxide, water, } \\
\text { materials from tanks; personnel injury due to } \\
\text { exposure to process waste, chemicals; release of } \\
\text { material outside facility; exposure to hot } \\
\text { chemicals; small release of material }\end{array}$ & 5 \\
\hline PPE (gloves) & Personnel injury due to burn & 4 \\
\hline PPE (gloves, safety glasses) & $\begin{array}{l}\text { Personnel injury due to burn; release of process } \\
\text { materials }\end{array}$ & 3 \\
\hline PPE (hard hats) & Personnel injury due to impact & 5 \\
\hline PPE (hearing protection) - if needed & Personnel injury due to high noise levels & 3 \\
\hline PSV on boiler & Large steam release & 4 \\
\hline PSV on chilled water jacket & Hot water release & 5 \\
\hline PSV on compressed air system & Personnel injury due to debris & 3 \\
\hline PSV on Feed Preparation tank & Tank failure and spill & 4 \\
\hline PSV on heat exchanger & Release of material from Hx heated loop & 6 \\
\hline PSV on permeate receipt tank & Tank failure and spill & 6 \\
\hline PSV on pulse pot & $\begin{array}{l}\text { Pressurized leak of permeate; personnel injury } \\
\text { due to debris }\end{array}$ & 3 \\
\hline PSV on receipt tank & Tank failure and spill & 6 \\
\hline PSV on Slurry Concentrate tank & Tank failure and spill & 6 \\
\hline PSV on the steam jacket & Steam release to facility & 2 \\
\hline PSV on Ultrafiltration Feed tank & Tank failure and spill & 4 \\
\hline Receipt tank level detection & $\begin{array}{l}\text { Spills of simulant, sodium hydroxide, water, } \\
\text { materials from other tanks; out of spec batch }\end{array}$ & 5 \\
\hline Receipt tank level detection and alarms & $\begin{array}{l}\text { Overflow of tanks into ventilation system; out of } \\
\text { spec batch }\end{array}$ & 3 \\
\hline Restricted Access & Personnel injury due to exposure to fumes & 4 \\
\hline Safety showers/eye wash & $\begin{array}{l}\text { Personnel injury due to exposure to material } \\
\text { (large release, splash) }\end{array}$ & 4 \\
\hline $\begin{array}{l}\text { Secondary confinement on sample archive } \\
\text { cabinet }\end{array}$ & Release of material outside facility & 1 \\
\hline Slurry Concentrate tank level detection & Spill of concentrated slurry, inhibited water & 5 \\
\hline $\begin{array}{l}\text { Slurry concentrate tank level detection and } \\
\text { alarm }\end{array}$ & $\begin{array}{l}\text { Overflow of tanks into ventilation system; spill } \\
\text { of concentrated slurry, inhibited water, nitric } \\
\text { acid, or simulant }\end{array}$ & 3 \\
\hline
\end{tabular}


Table 5.1 (Contd)

\begin{tabular}{|c|c|c|}
\hline $\begin{array}{l}\text { Safeguards Selected for the PEP at Final } \\
\text { Hazard Analysis }\end{array}$ & Type(s) of Hazardous Condition Addressed & $\begin{array}{l}\text { Highest } \\
\text { Risk } \\
\text { Score } \\
\text { Applied }\end{array}$ \\
\hline Speed limits (PNNL) & $\begin{array}{l}\text { Personnel injury due to exposure to fumes, } \\
\text { vehicle impact; release of material outside } \\
\text { facility }\end{array}$ & 4 \\
\hline Sprinkler system & Facility fire; personnel injury due to fire & 3 \\
\hline $\begin{array}{l}\text { Stop or bore through on the damper to } \\
\text { prevent full closure }\end{array}$ & Tank failure and spill & 6 \\
\hline UL listed heat trace & & 5 \\
\hline Ultrafiltration feed tank level detection & $\begin{array}{l}\text { Spills of simulant, sodium hydroxide, water, } \\
\text { materials from other tanks; carryover of material } \\
\text { into ventilation system }\end{array}$ & 4 \\
\hline $\begin{array}{l}\text { Ultrafiltration feed tank level detection and } \\
\text { alarms }\end{array}$ & $\begin{array}{l}\text { Spills of simulant, sodium hydroxide, water, } \\
\text { materials from other tanks; out of spec batch }\end{array}$ & 3 \\
\hline Vacuum breaker on steam jacket & Collapse of steam jacket & 2 \\
\hline
\end{tabular}




\subsection{References}

AIChE. 1992. Guidelines for Hazard Evaluation Procedures, Vol. 2. American Institute of Chemical Engineers, New York.

Pacific Northwest National Laboratory (PNNL). September 24, 2007. Sampler for T01A/B Drawing Worksheet. PNNL, Richland, Washington.

Bechtel National, Inc. 2002. Performance Requirements for Engineering Scale Pretreatment System. 24590-PTF-3YD-UFP-00002, Rev. 1, Bechtel National Inc., Richland Washington.

TKS. May 17, 2007a. Process Flow Diagram. Drawing No. 007L0100-106, Tessenderlo Kerley Services, ESPS, Richland, Washington.

TKS. June 01, 2007b. Piping and Instrumentation Diagram. Drawing No. 007Z001-22, Tessenderlo Kerley Services, ESPS, Richland, Washington, Revision M.

TKS. September 28, 2007c. Piping and Instrumentation Diagram. Drawing No. 007Z001-25, Tessenderlo Kerley Services, PEP, Richland, Washington, Revision 3.

TKS. September 28, 2007c. Piping and Instrumentation Diagram. Drawing No. 007Z001-35, Tessenderlo Kerley Services, PEP, Richland, Washington, Revision 7.

TKS. June 04, 2007d. ESPS General Arrangement. Drawing No. 007L001-5, Tessenderlo Kerley Services, ESPS, Richland, Washington. 
Appendix A

Meeting Attendees 


\section{PEP 30\% Hazard Identification and System Nodalization}

5/29, 2007

Richland, WA

Nick Barilo
John Geeting
Bob Gough
Gary Harvey
Dwight Hughes
Gary Josephson
Peter Keegan
Wes Lawrence
Chris Musick
Don Quilici
Randy Richardson
Bob Smoter
Brad Stiver
Robin Sullivan
Steve Wright
Jonathan Young

\section{Name}

$\quad$ Company
PNNL
PNNL
PNNL
WGI
PNNL
PNNL
EDD
PNNL
BNI
PNNL
WGI
PNNL
BNI
PNNL
Energy Solutions
PNNL

PEP 30\% HAZOP

6/04-6/06, 2007

Carlsbad, NM

Scott Bierle

Name

Company

John Geeting

Gary Harvey

Gary Josephson

Peter Keegan

Robert Kelly

Loren Kirkes

Wes Lawrence

Chris Musick

Randy Richardson

Brad Stiver

Robin Sullivan

Jonathan Young

TKS

PNNL

WGI

PNNL

EDD

TKS

TKS

PNNL

BNI

WGI

BNI

PNNL

PNNL 
PEP 30\% PHA

6/19-6/21, 2007

Richland, WA

Tom Davis

Bob Gough

Gary Harvey

Dwight Hughes

Gary Josephson

Darrell LaMastus

Wes Lawrence

Don Quilici

Craig Smith

Brad Stiver

Robin Sullivan

Jonathan Young

John Geeting

Gary Harvey

Dwight Hughes

Joel Kirkes

Don Quilici

Steve Smith

Brad Stiver

Robin Sullivan

Jonathan Young
Name

Company
PNNL
PNNL
WGI
PNNL
PNNL
WGI
PNNL
PNNL
TFE
BNI
PNNL
PNNL

PEP 90\% HA

10/11, 2007

Richland, WA

Name

Company
PNNL
WGI
PNNL
TKS
PNNL
PNNL
BNI
PNNL
PNNL

PEP 100\% Gap Analysis

3/10, 2008

Richland, WA

\begin{tabular}{lc}
\multicolumn{1}{c}{ Name } & Company \\
John Geeting & PNNL \\
Robin Sullivan & PNNL \\
Jonathan Young & PNNL
\end{tabular}




\begin{tabular}{lc}
\multicolumn{2}{c}{ PEP 100\% Procedure Review } \\
4/01, 2007 \\
Richland, WA \\
Name & Company \\
John Geeting & PNNL \\
Gary Harvey & WGI \\
Gary Josephson & PNNL \\
Robin Sullivan & PNNL \\
Jonathan Young & PNNL
\end{tabular}

PEP 100\% HA

4/15 \& 4/17, 2007

Richland, WA

\begin{tabular}{lc}
\multicolumn{1}{c}{ Name } & Company \\
Francis Buck & PNNL \\
John Geeting & PNNL \\
Gary Harvey & WGI \\
MD Hughes & PNNL \\
Gary Josephson & PNNL \\
Joel Kirkes & TKS \\
Scott Lehrman & WTP \\
Don Quilici & PNNL \\
Gary Sevigny & PNNL \\
Steve Smith & PNNL \\
Brad Stiver & BNI \\
Robin Sullivan & PNNL \\
John Truax & WGI \\
Steve Wright & BNI \\
Jonathan Young & PNNL
\end{tabular}


Appendix B

Hazard Analysis Nodalization 


\section{B.1 Final Nodalization Completed for Hazard Analysis}

\begin{tabular}{|c|c|}
\hline Node & Description \\
\hline \multirow[t]{4}{*}{01} & $\begin{array}{l}\text { Process Feed/Receipt Vessels. Tanks including transfer pumps up to tank UFP-VSL-T01 A/B } \\
\text { (includes material addition lines/connections) (P\&ID drawing 1,2, and 9) }\end{array}$ \\
\hline & HLP-VSL-T22 with steam (representative of the other node 1 tanks) (P\&ID drawing 1) \\
\hline & FEP-VSL-T01 (P\&ID drawing 9) \\
\hline & FRP-VSL-T01 (P\&ID drawing 2) \\
\hline \multirow[t]{3}{*}{02} & Feed Preparation. UFP-VSL-T01 A/B tanks through heat exchangers (P\&ID drawing 3, 4, 5) \\
\hline & Pulse jet mixers (P\&ID drawing 16,17) \\
\hline & Steam injection \\
\hline \multirow[t]{3}{*}{03} & $\begin{array}{l}\text { Ultrafiltration Feed Vessel. UFP-VSL-T02A up to suction side of solids transfer pump and } \\
\text { suction side of UFP-PMP-T42A, including ejector. (P\&ID drawing 6) }\end{array}$ \\
\hline & Pulse jet mixers (P\&ID drawing 18) \\
\hline & Steam injection \\
\hline \multirow[t]{3}{*}{04} & $\begin{array}{l}\text { Ultrafiltration Loop. Ultrafiltration system from UFP-PMP-T42A back to tank UFP-VSL- } \\
\text { T02A and up to the permeate evaporator tanks. }\end{array}$ \\
\hline & Pumps, pulse pots/ultrafilter (P\&ID drawings 6, 7) \\
\hline & Heat exchanger and lines ((P\&ID drawing 5) \\
\hline 05 & $\begin{array}{l}\text { Slurry Concentrate Waste. Solids transfer pump into HLP-VSL-T27 and portable pump for } \\
\text { emptying into drums. (P\&ID drawing 10) }\end{array}$ \\
\hline \multirow[t]{3}{*}{06} & $\begin{array}{l}\text { Permeate Collection Tanks. Tanks and pump between T62A and T62B. (Note: Previously } \\
\text { referred to as Permeate Evaporator Tanks for 30\% analysis) (P\&ID drawing 8) }\end{array}$ \\
\hline & UFP-VSL-T62A with steam (representative of the other node 6 tank) \\
\hline & UFP-VSL-T62B \\
\hline 07 & Off-Gas System. (P\&ID drawing 11) \\
\hline 08 & Steam System and Condensate Drains. (P\&ID drawing 13) \\
\hline 09 & Chemical Addition and RO System. (P\&ID drawings 14 and 15) \\
\hline 10 & Package Chiller. Including accumulator tank (P\&ID drawing 25) \\
\hline 11 & $\begin{array}{l}\text { Compressed/High Pressure Air. Including amplifier (P\&ID drawing 12). NOTE: There is } \\
\text { also an accumulator tank on this system not shown on the drawing }\end{array}$ \\
\hline 12 & Vacuum System. Including the cyclone separator (P\&ID drawing 12) \\
\hline 13 & Sampling System. (P\&ID drawings 20, 21 and 22) \\
\hline A & $\begin{array}{l}\text { Operations } \\
\text { 1. Tank filling (manual, locally monitored) } \\
\text { 2. Transfers (valve alignment, pump hookup) } \\
\text { 3. Process monitoring } \\
\text { 4. Process utility operations (HVAC, steam, chilled water, vacuum system) } \\
\text { 5. Facility building operations (HVAC) }\end{array}$ \\
\hline
\end{tabular}




\begin{tabular}{|c|c|}
\hline Node & Description \\
\hline B & 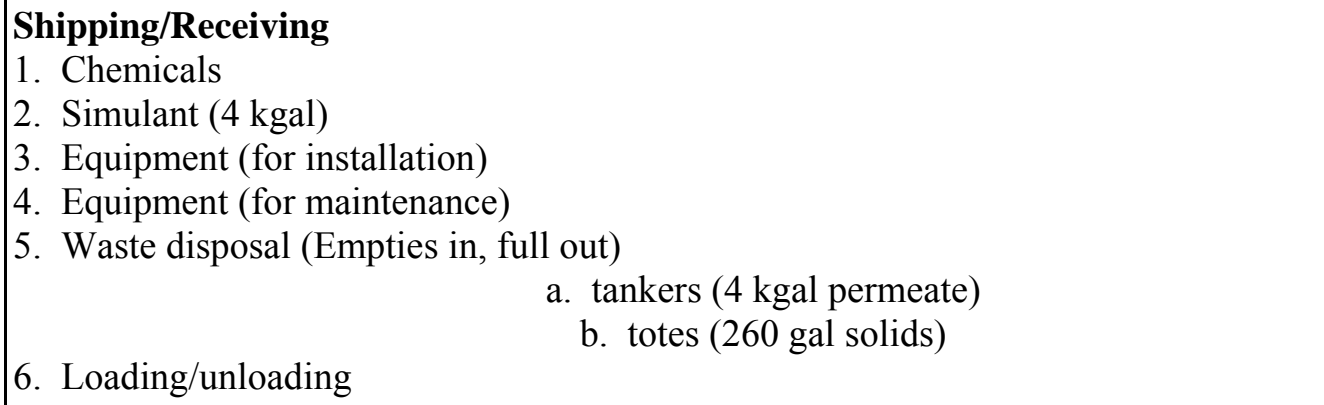 \\
\hline C & $\begin{array}{l}\text { Chemical Addition } \\
\text { 1. Local, manual operation } \\
\text { 2. Addition at tank } \\
\text { 3. Sampling at addition tank (acquire sample and transfer to testing location) } \\
\text { Note: Need to address assumption about concentration of chemicals received at PDL) }\end{array}$ \\
\hline $\mathbf{D}$ & $\begin{array}{l}\text { Sampling } \\
\text { 1. Sample acquisition } \\
\text { 2. Sample analysis (locally in facility-centrifuging, solids testing, } \mathrm{pH} \text { test) } \\
\text { 3. Sample storage/archive in cabinets }\end{array}$ \\
\hline $\mathbf{E}$ & $\begin{array}{l}\text { Start-up/Maintenance } \\
\text { 1. Shake-down testing } \\
\text { a. process tests } \\
\text { b. utility tests (electrical, air, water, HVAC, steam, vacuum) } \\
\text { 2. Upset response } \\
\text { a. recovery (e.g., overfill, plugged line) } \\
\text { b. spill recovery } \\
\text { c. off-normal process clean-up } \\
\text { d. off-normal HVAC clean-up } \\
\text { 3. Routine maintenance } \\
\text { a. process vent cleaning } \\
\text { b. HVAC cleaning } \\
\text { 4. Repair } \\
\text { a. ultrafilter tube replacement } \\
\text { b. equipment repair (e.g., Agitator remove and replace) } \\
\text { c. facility equipment repair } \\
\text { d. steam trap repair }\end{array}$ \\
\hline $\mathbf{F}$ & $\begin{array}{l}\text { Temporary Storage } \\
\text { 1. Transfer process waste from tanks into containers } \\
\text { 2. Load containers into } 90 \text { day storage (new and spent materials) } \\
\text { 3. Store containers }\end{array}$ \\
\hline
\end{tabular}


B.2 PEP 100\% Design Drawings with Final System Nodes Identified

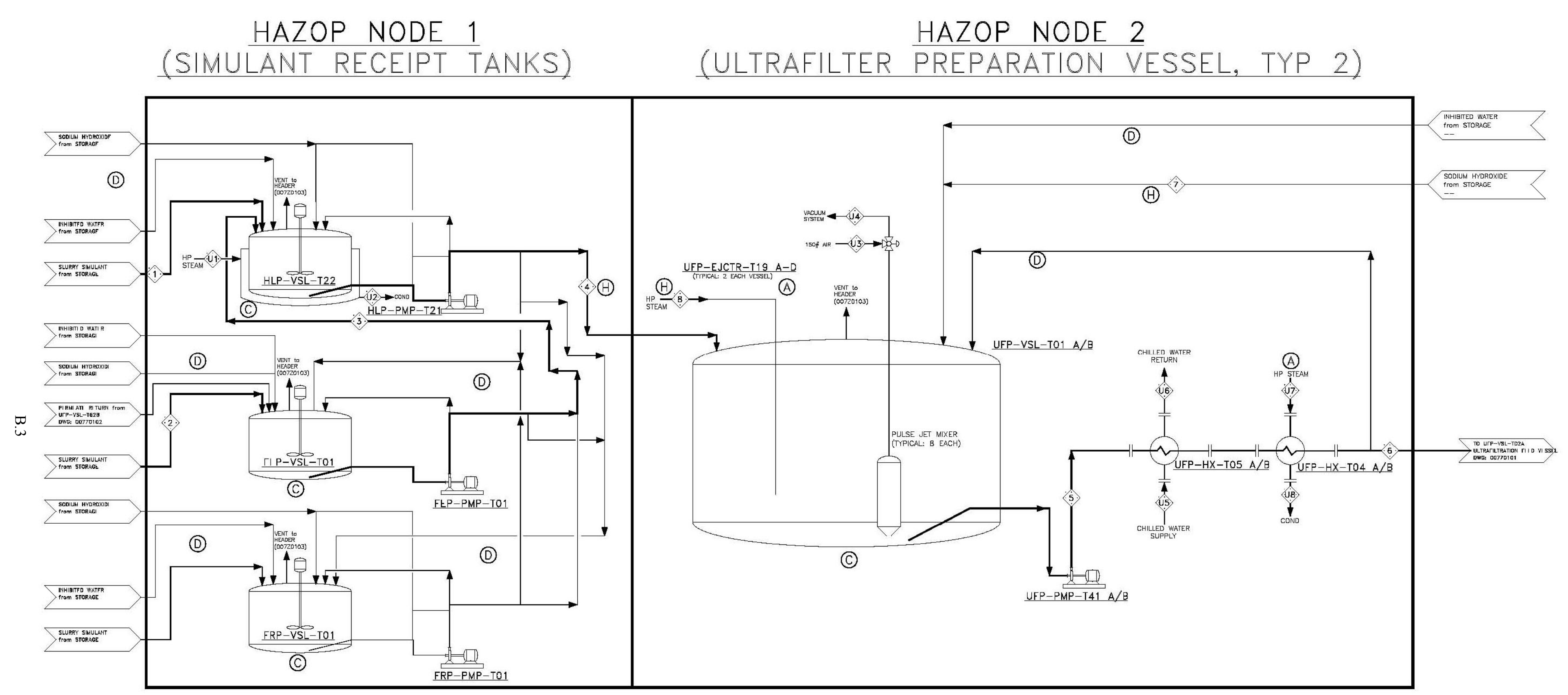


HAZOP NODE 3

\section{(ULTRAFILTER FEED VESSEL)}

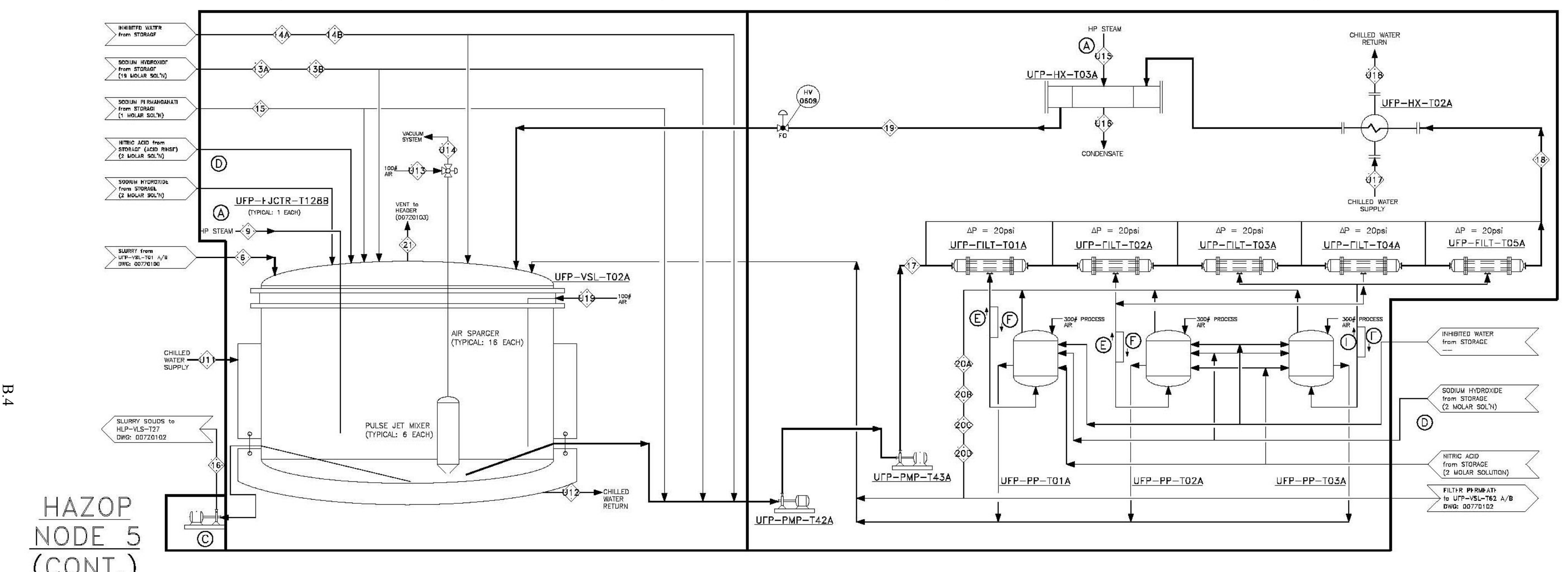

HAZOP NODE 4

(ULTRAFILTRATION SYSTEM)
(CONT.) 
HAZOP NODE 6

(PERMEATE RECEIPT TANKS)

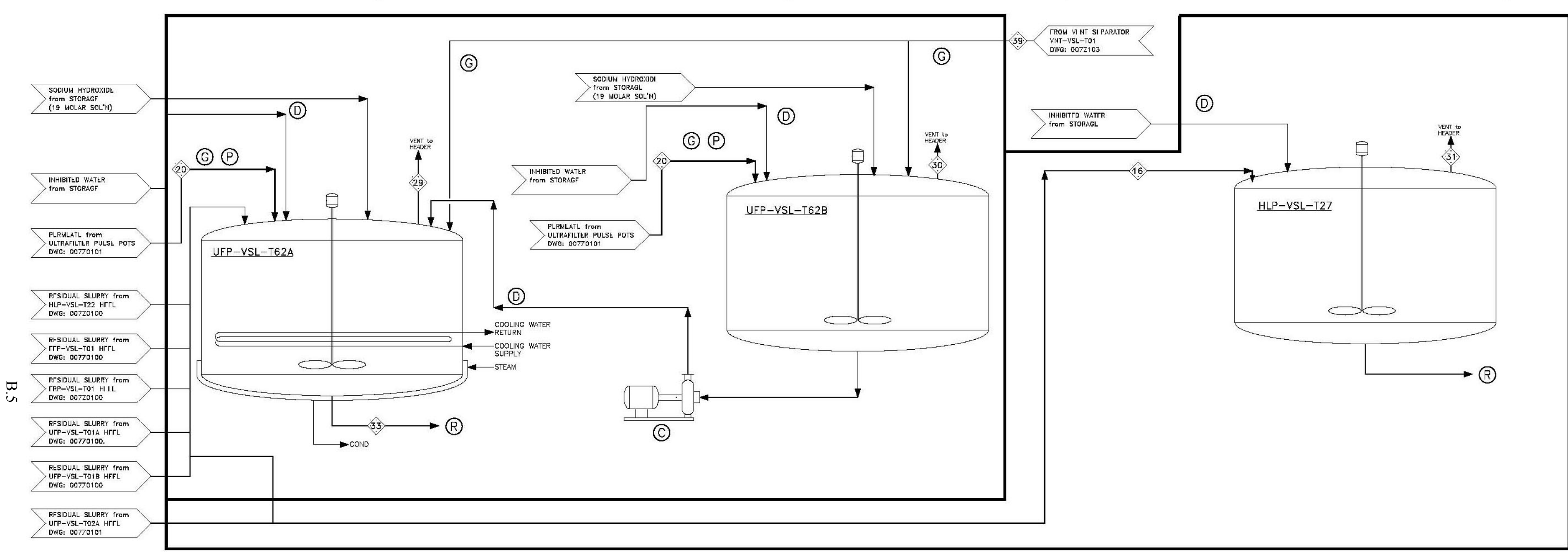


HAZOP NODE 7

(VENTILATION SYSTEM)

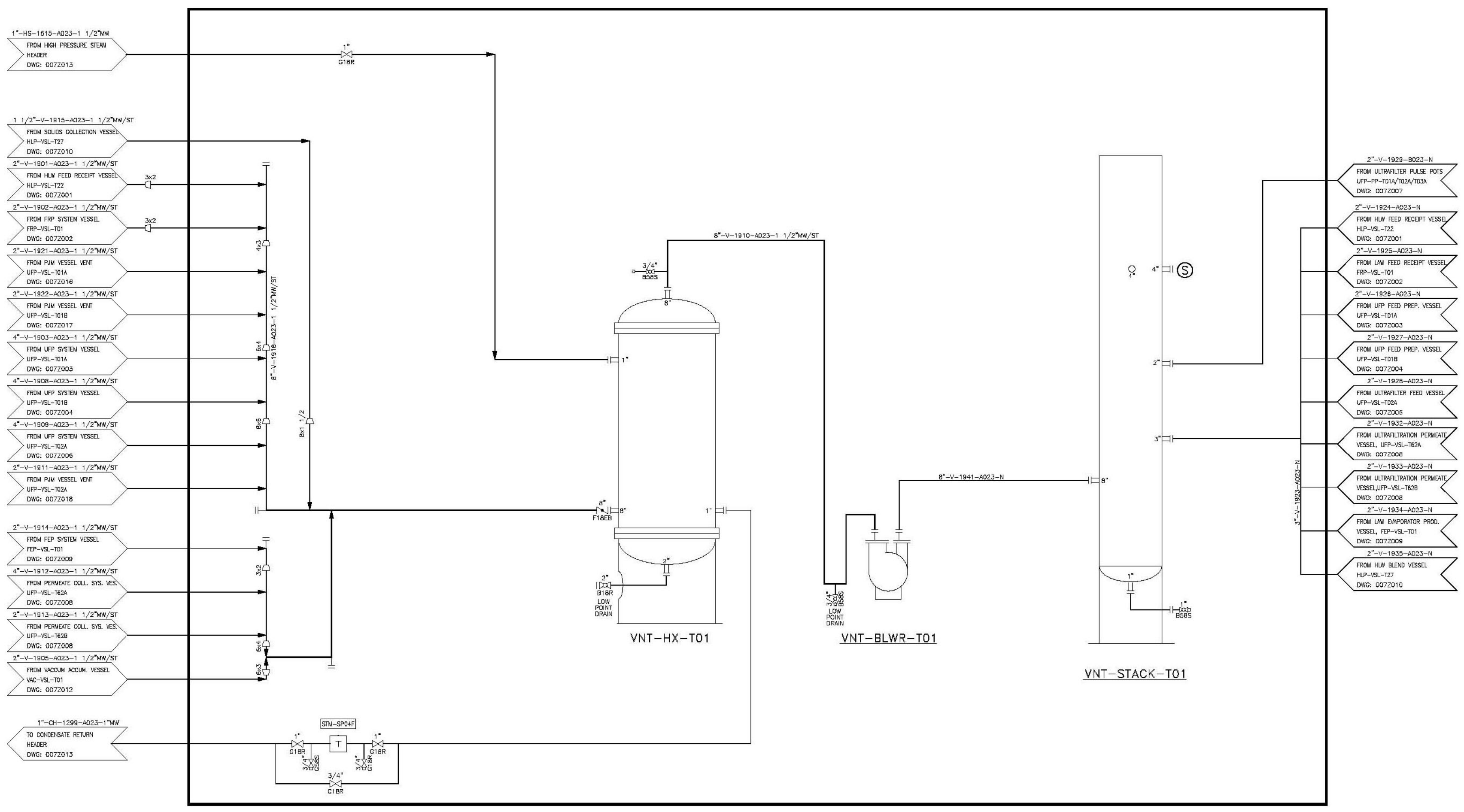



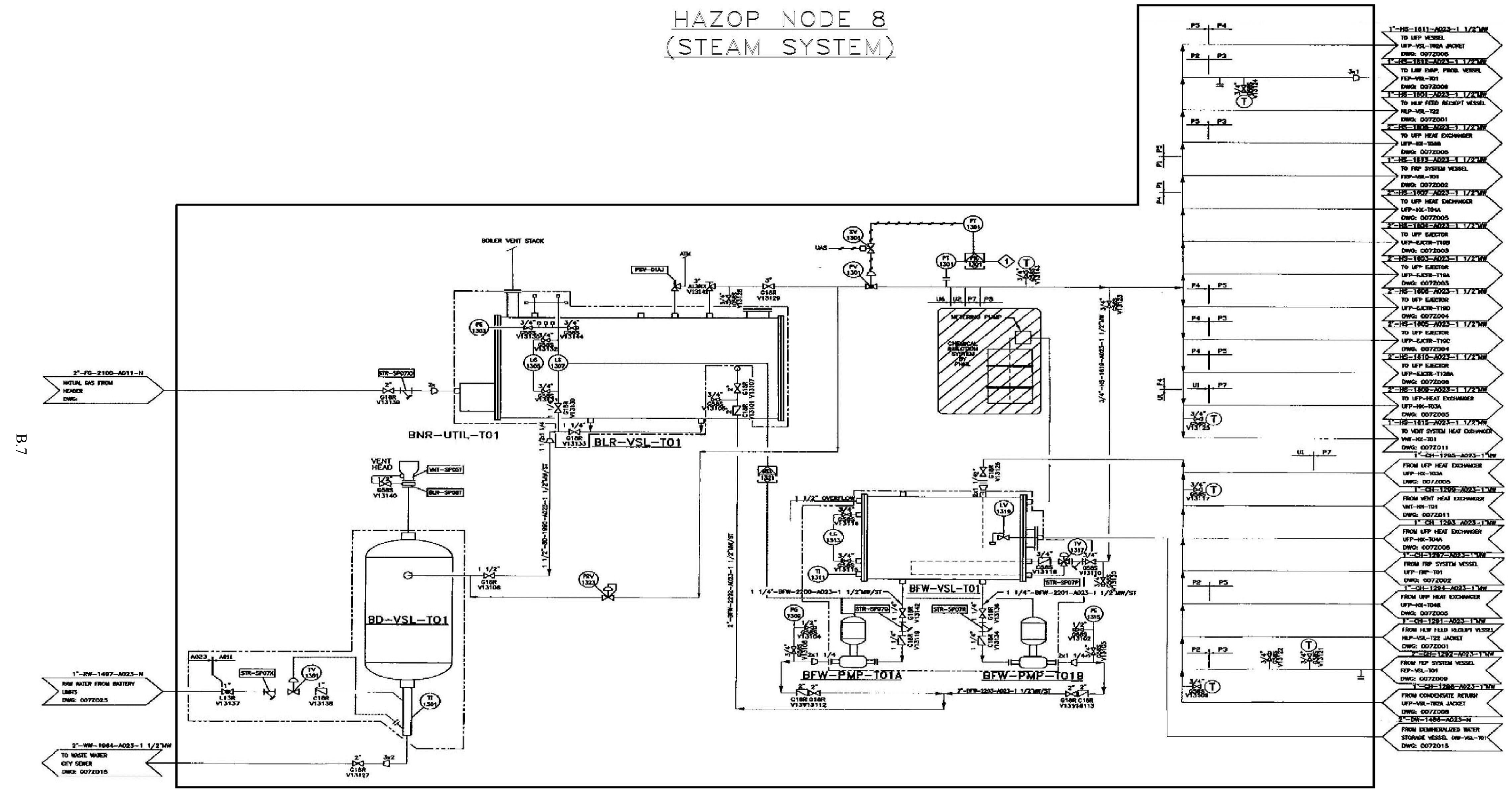


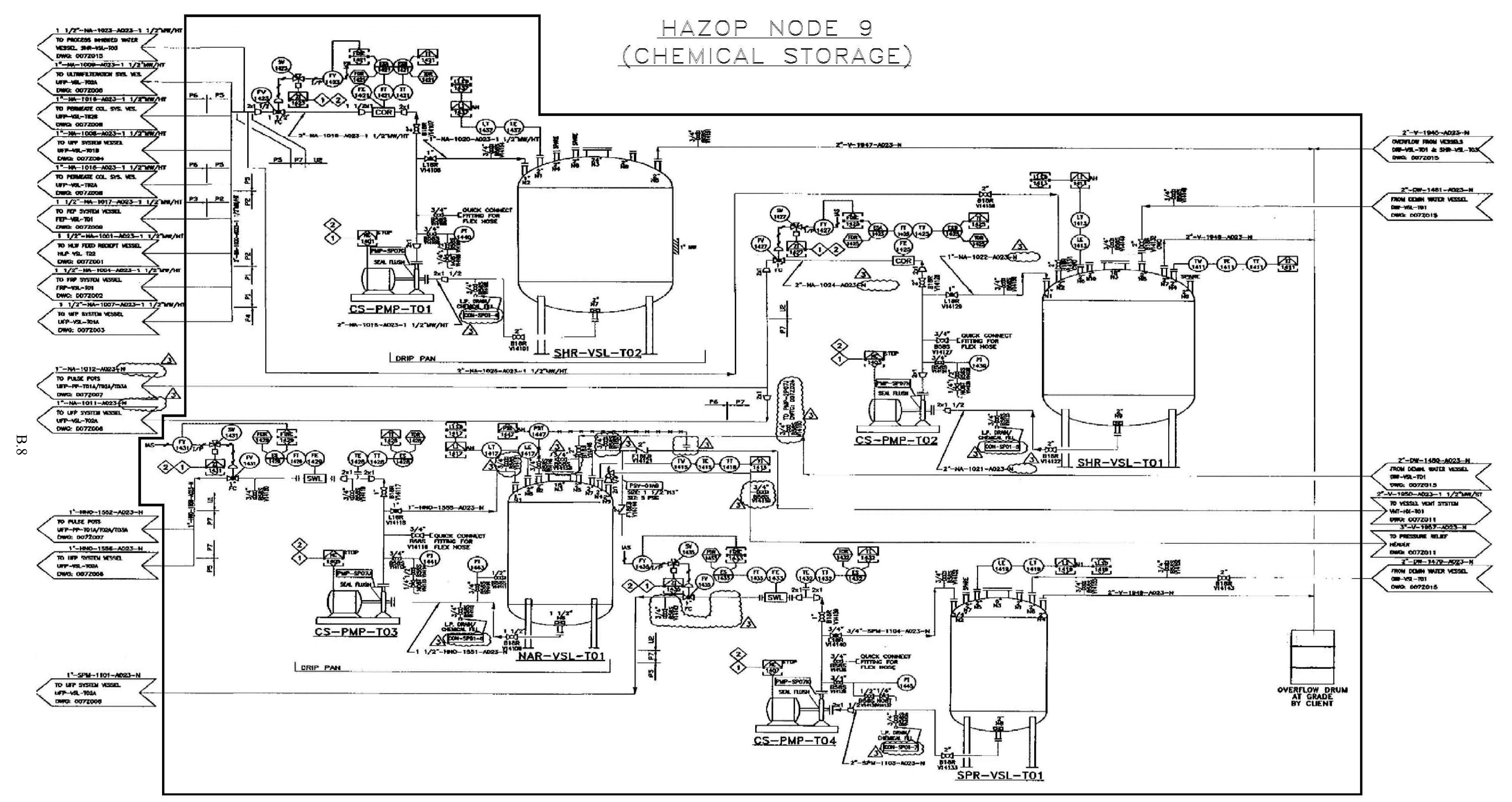









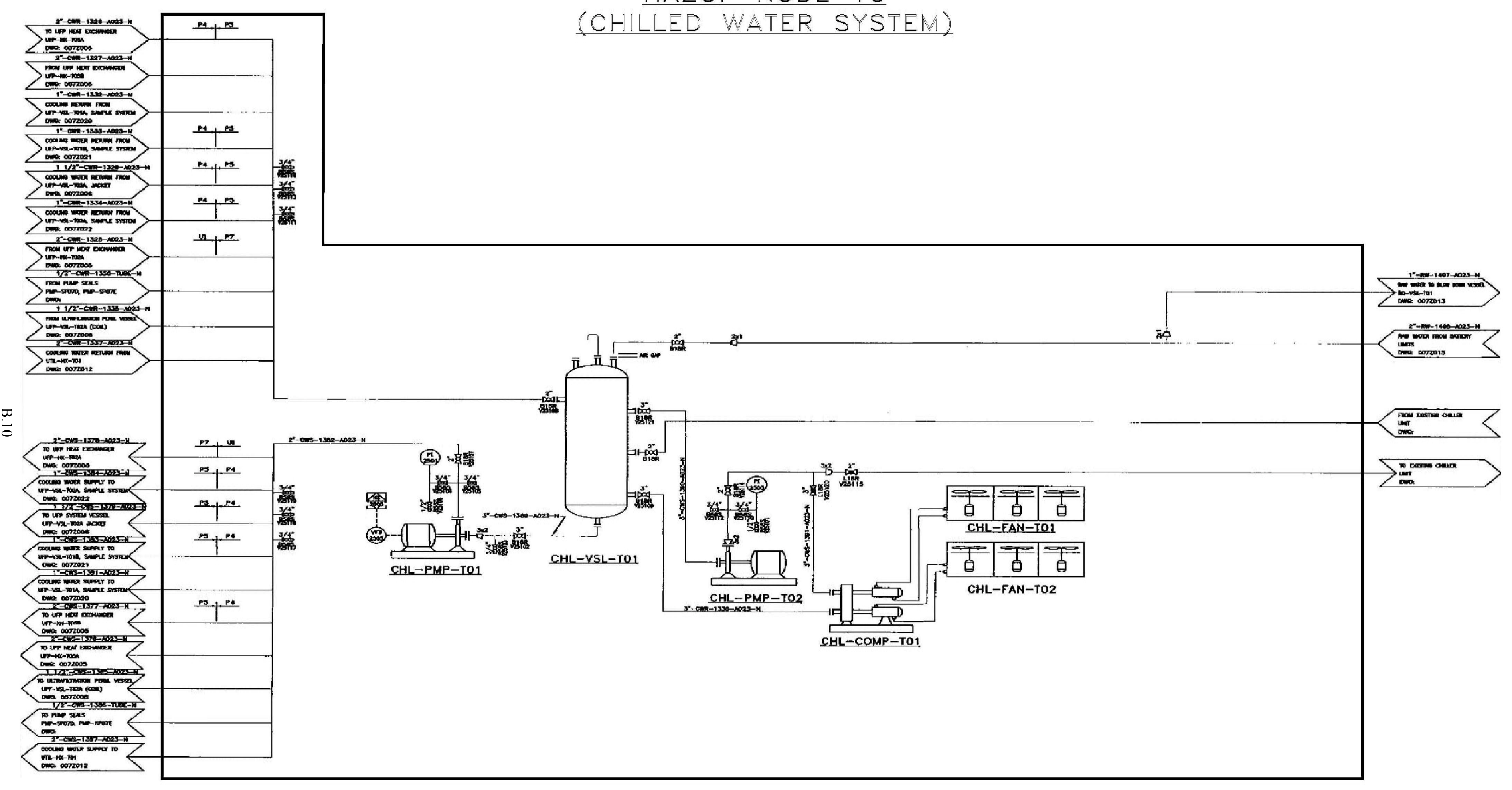




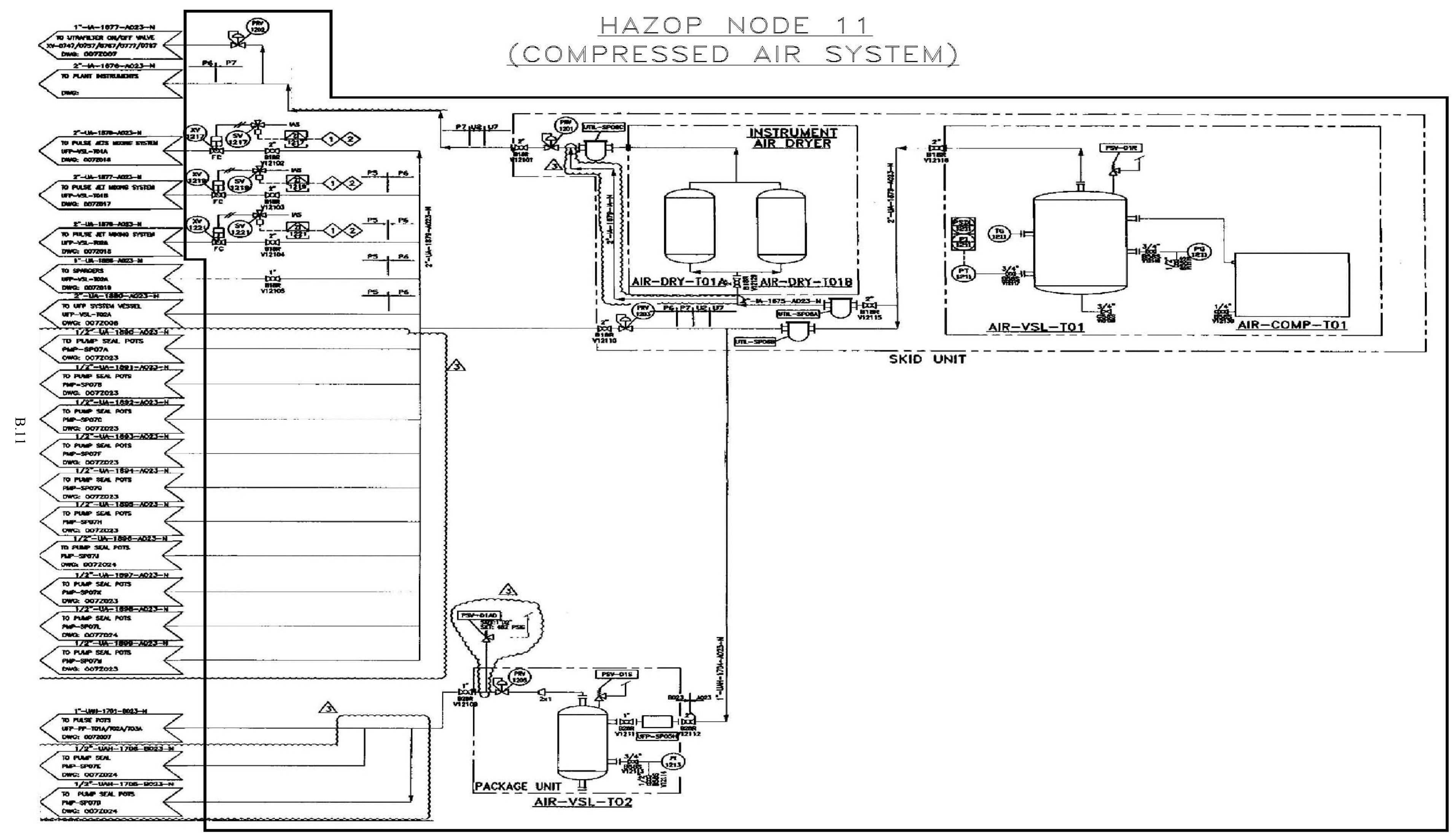




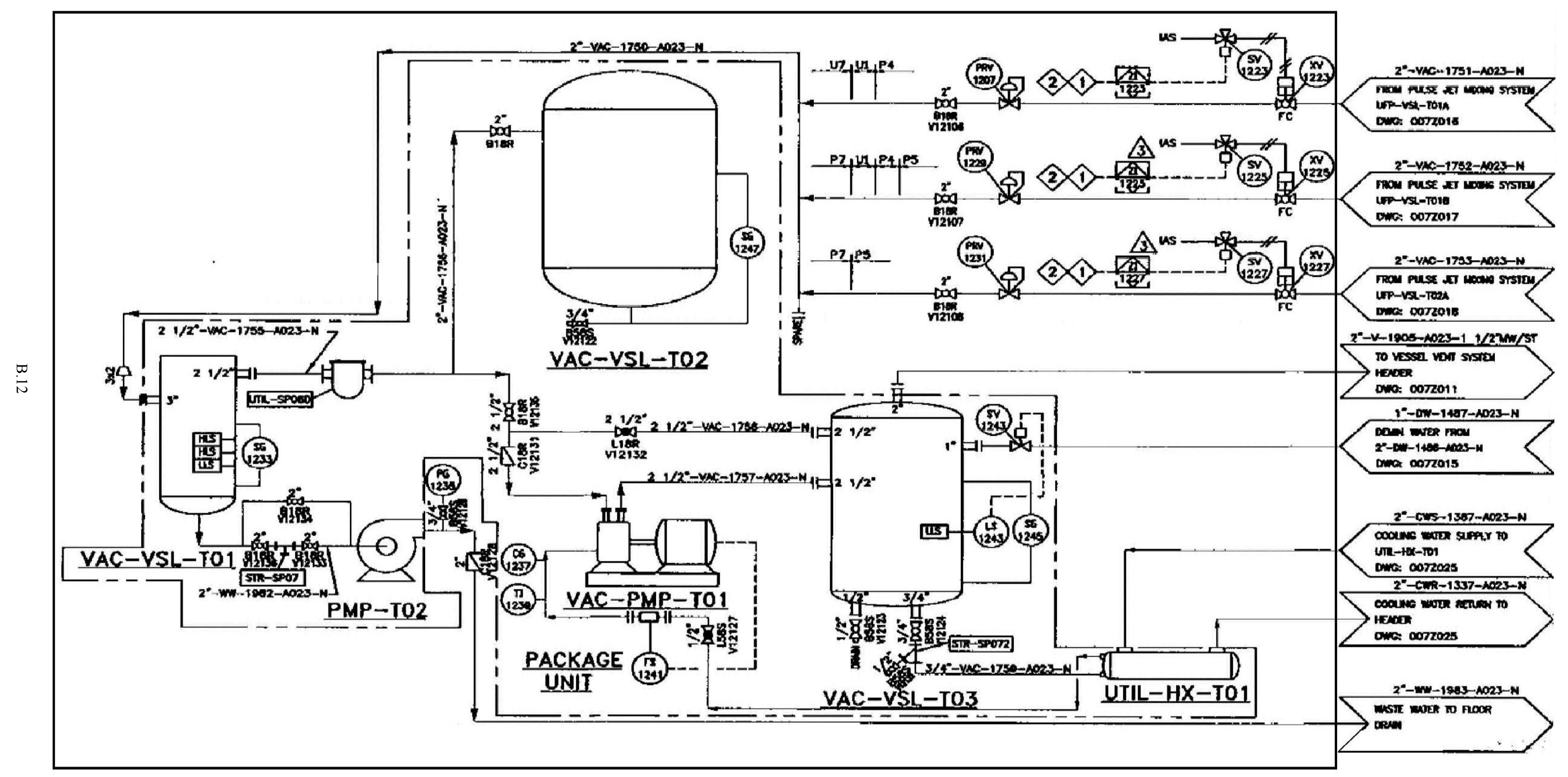




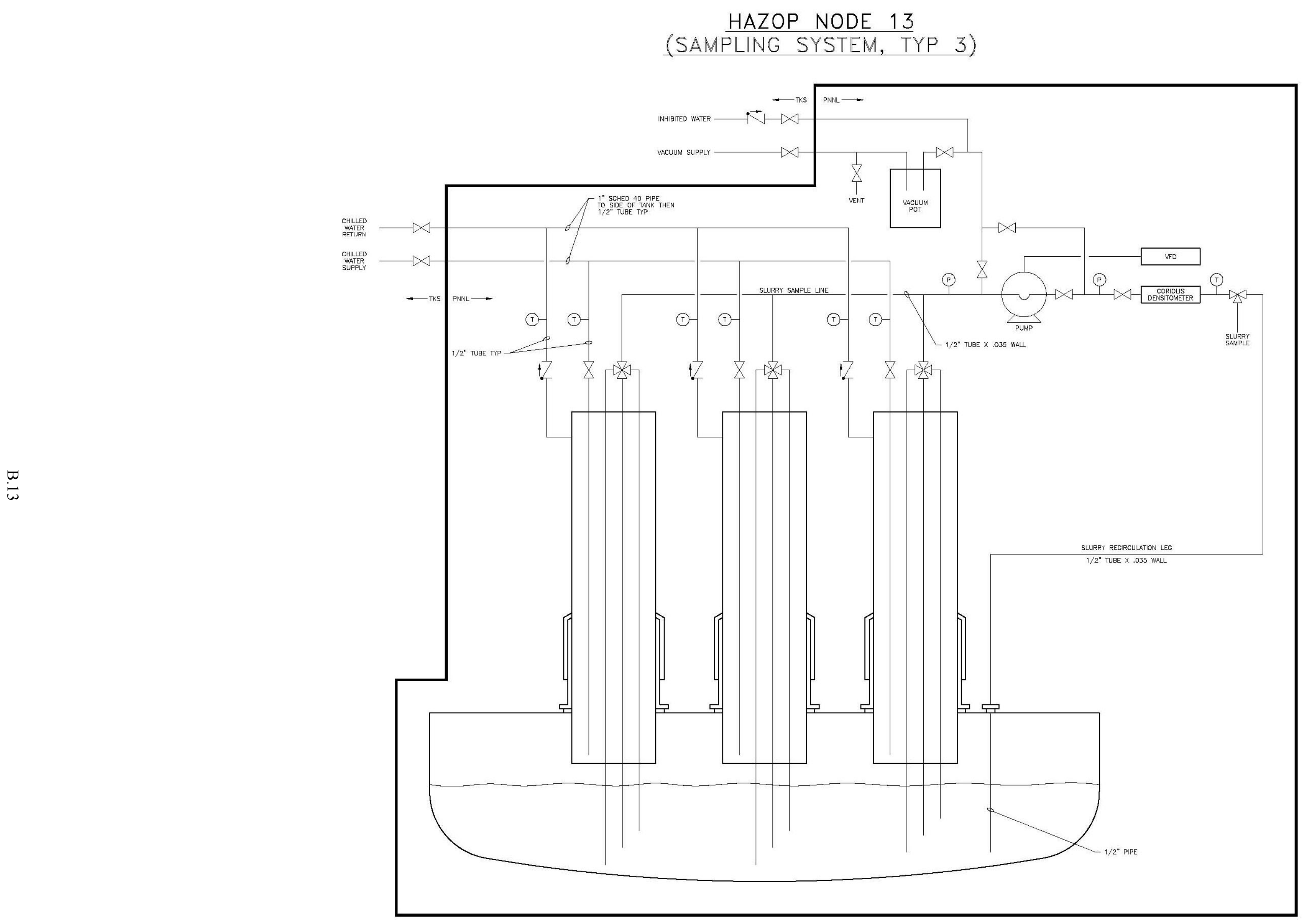


Appendix C

HAZOP Deviation Guide 
Table C.1. HAZOP Deviation Guide

\begin{tabular}{|c|c|c|c|c|c|c|c|}
\hline \multirow{2}{*}{$\begin{array}{c}\text { Process } \\
\text { Variables }\end{array}$} & \multicolumn{7}{|c|}{ Guide Words } \\
\hline & $\begin{array}{l}\text { No, Not, } \\
\text { None }\end{array}$ & $\begin{array}{l}\text { Less, Low, } \\
\text { Short }\end{array}$ & More, High, & Part of & $\begin{array}{c}\text { As Well as, } \\
\text { Also }\end{array}$ & Other Than & Reverse \\
\hline Flow & No flow & $\begin{array}{l}\text { Low rate, } \\
\text { Low total }\end{array}$ & $\begin{array}{l}\text { High rate, } \\
\text { High total }\end{array}$ & $\begin{array}{l}\text { Missing } \\
\text { ingredient }\end{array}$ & $\begin{array}{l}\text { Misdirection, } \\
\text { contamination, } \\
\text { impurities }\end{array}$ & $\begin{array}{l}\text { Wrong } \\
\text { material }\end{array}$ & Backflow \\
\hline Pressure & $\begin{array}{l}\text { Open to } \\
\text { atmosphere }\end{array}$ & Low pressure & $\begin{array}{l}\text { High } \\
\text { pressure }\end{array}$ & & & & Vacuum \\
\hline Temperature & Freezing & $\begin{array}{l}\text { Low } \\
\text { temperature }\end{array}$ & $\begin{array}{l}\text { High } \\
\text { temperature }\end{array}$ & & & & $\begin{array}{l}\text { Auto- } \\
\text { refrigeration }\end{array}$ \\
\hline Level & Empty & Low level & High level & $\begin{array}{l}\text { Low } \\
\text { interface }\end{array}$ & High interface & & \\
\hline Agitation & No mixing & Poor mixing & $\begin{array}{l}\text { Excessive } \\
\text { mixing }\end{array}$ & \begin{tabular}{|l|} 
Mixing \\
interruption
\end{tabular} & Foaming & & $\begin{array}{l}\text { Phase } \\
\text { separation }\end{array}$ \\
\hline Reaction & No reaction & $\begin{array}{l}\text { Slow } \\
\text { reaction }\end{array}$ & $\begin{array}{l}\text { Runaway } \\
\text { reaction }\end{array}$ & $\begin{array}{l}\text { Partial } \\
\text { reaction }\end{array}$ & Side reaction & $\begin{array}{l}\text { Wrong } \\
\text { reaction }\end{array}$ & Decomposition \\
\hline Time procedure & $\begin{array}{l}\text { Skipped or } \\
\text { missing step }\end{array}$ & $\begin{array}{l}\text { Too short, } \\
\text { Too little }\end{array}$ & $\begin{array}{l}\text { Too long, } \\
\text { Too much }\end{array}$ & $\begin{array}{l}\text { Action(s) } \\
\text { skipped }\end{array}$ & $\begin{array}{l}\text { Extra action(s) } \\
\text { (shortcuts) }\end{array}$ & $\begin{array}{l}\text { Wrong } \\
\text { action }\end{array}$ & $\begin{array}{l}\text { Out of order, } \\
\text { opposite }\end{array}$ \\
\hline Speed & Stopped & Too slow & Too fast & $\begin{array}{l}\text { Out of } \\
\text { synch }\end{array}$ & & \begin{tabular}{|l|} 
Web or belt \\
break
\end{tabular} & Backward \\
\hline $\begin{array}{l}\text { Composition/ } \\
\text { Concentration }\end{array}$ & $\begin{array}{l}\text { Missing } \\
\text { ingredient }\end{array}$ & \begin{tabular}{|l|} 
Less \\
ingredient/ \\
low \\
concentration
\end{tabular} & $\begin{array}{l}\text { More } \\
\text { ingredient/ } \\
\text { high } \\
\text { concentration }\end{array}$ & $\begin{array}{l}\text { Missing } \\
\text { ingredient }\end{array}$ & $\begin{array}{l}\text { Contaminant/ } \\
\text { additional } \\
\text { ingredient }\end{array}$ & $\begin{array}{l}\text { Wrong } \\
\text { ingredient }\end{array}$ & \\
\hline $\mathrm{Ph}$ & & Low ph & High ph & & & & \\
\hline Viscosity & & $\begin{array}{l}\text { Low } \\
\text { viscosity }\end{array}$ & $\begin{array}{l}\text { High } \\
\text { viscosity }\end{array}$ & & & & \\
\hline Voltage & No voltage & Low voltage & High voltage & $\begin{array}{l}\text { Wrong } \\
\text { waveform }\end{array}$ & $\begin{array}{l}\text { Interference } \\
\text { voltage, } \\
\text { induced } \\
\text { voltage }\end{array}$ & \begin{tabular}{|l|} 
Wrong \\
frequency, \\
AC instead \\
of DC \\
DC instead \\
of AC
\end{tabular} & Wrong polarity \\
\hline Current & No current & Low current & High current & & & $\begin{array}{l}\text { Current } \\
\text { fluctuating }\end{array}$ & Wrong polarity \\
\hline Static charge & & & $\begin{array}{l}\text { Static charge } \\
\text { present }\end{array}$ & & & & \\
\hline $\begin{array}{l}\text { Structural } \\
\text { integrity }\end{array}$ & $\begin{array}{l}\text { Structural } \\
\text { failure }\end{array}$ & $\begin{array}{l}\text { Less } \\
\text { integrity }\end{array}$ & $\begin{array}{l}\text { More } \\
\text { integrity }\end{array}$ & & & & \\
\hline Shielding & & $\begin{array}{l}\text { Less } \\
\text { Shielding }\end{array}$ & $\begin{array}{l}\text { More } \\
\text { Shielding }\end{array}$ & Streaming & Bremsstrahlung & $\begin{array}{l}\text { Wrong type } \\
\text { of shielding }\end{array}$ & \\
\hline Confinement & $\begin{array}{l}\text { No } \\
\text { confinement }\end{array}$ & $\begin{array}{l}\text { Degraded } \\
\text { confinement }\end{array}$ & \begin{tabular}{|l|} 
Excessive \\
confinement
\end{tabular} & & & $\begin{array}{l}\text { Bypass } \\
\text { pathway }\end{array}$ & \\
\hline Special & $\begin{array}{l}\text { Utility } \\
\text { failure }\end{array}$ & External leak & $\begin{array}{l}\text { External } \\
\text { rupture }\end{array}$ & Tube leak & Tube rupture & \begin{tabular}{|l|} 
Startup, \\
shutdown, \\
maintenance
\end{tabular} & \\
\hline
\end{tabular}


Appendix D

Hazard Identification Checklist 
Table D.1. 90\% PEP Hazard Identification Checklist

\begin{tabular}{|c|c|c|c|}
\hline Type of Hazard & Form of Hazard & $\mathbf{X}$ & Remarks \\
\hline \multirow{15}{*}{$\begin{array}{l}\text { A. Electrical } \\
\text { Note: All electrical } \\
\text { hazards are standard } \\
\text { industrial hazards with the } \\
\text { exception of loss of } \\
\text { power, which is covered } \\
\text { by external events (see } \\
\text { hazard P.4) }\end{array}$} & 1. Battery banks & & \\
\hline & 2. Diesel units & & The design at this time does not include any backup power \\
\hline & 3. High voltage lines & $\mathrm{X}$ & Underground 480 supply \\
\hline & 4. Transformers & $\mathrm{X}$ & High Bay \\
\hline & 5. Wiring & $\mathrm{X}$ & \\
\hline & 6. Switchgear & $\mathrm{X}$ & \\
\hline & $\begin{array}{l}\text { 7. Underground } \\
\text { wiring }\end{array}$ & $\mathrm{X}$ & \\
\hline & 8. Cable runs & $\mathrm{X}$ & \\
\hline & $\begin{array}{l}\text { 9. Service outlets } \\
\text { and fittings }\end{array}$ & $\mathrm{X}$ & \\
\hline & $\begin{array}{l}\text { 10. Electric motor } \\
\text { driven pumps }\end{array}$ & $\mathrm{X}$ & \\
\hline & 11. Other motors & $\mathrm{X}$ & Agitators, HVAC fans, HP compressor \\
\hline & 12. Heaters & $\mathrm{X}$ & $\begin{array}{l}\text { Building heaters are natural gas, Heat tracing on the caustic } \\
\text { addition lines (both systems) }\end{array}$ \\
\hline & 13. Power tools & $\mathrm{X}$ & Maintenance and installation \\
\hline & 14. Hoists & $\mathrm{X}$ & 20 ton bridge crane \\
\hline & 15. Other & $\mathrm{X}$ & Static discharge \\
\hline \multirow[t]{15}{*}{ B. Nuclear Criticality } & 1. Vaults & & N/A \\
\hline & $\begin{array}{l}\text { 2. Temporary } \\
\text { storage areas }\end{array}$ & & $\mathrm{N} / \mathrm{A}$ \\
\hline & 3. Receiving areas & & $\mathrm{N} / \mathrm{A}$ \\
\hline & 4. Casks & & $\mathrm{N} / \mathrm{A}$ \\
\hline & 5. Burial grounds & & N/A \\
\hline & 6. Storage tanks & & $\mathrm{N} / \mathrm{A}$ \\
\hline & 7. Storage racks & & $\mathrm{N} / \mathrm{A}$ \\
\hline & 8. Canals and basins & & N/A \\
\hline & 9. Decon solution & & N/A \\
\hline & $\begin{array}{l}\text { 10. Trucks, forklifts, } \\
\text { dollies }\end{array}$ & & N/A \\
\hline & 11. Hand carry & & $\mathrm{N} / \mathrm{A}$ \\
\hline & 12. Cranes/lifts & & N/A \\
\hline & $\begin{array}{l}\text { 13. Hot cells, } \\
\text { assembly }\end{array}$ & & $\mathrm{N} / \mathrm{A}$ \\
\hline & 14. Inspection areas & & N/A \\
\hline & 15. Other & & $\mathrm{N} / \mathrm{A}$ \\
\hline \multirow[t]{3}{*}{ C. Kinetic/ Linear } & 1. Cars/trucks/buses & $\mathrm{X}$ & Traffic next to building (e.g., material delivery, waste disposition) \\
\hline & $\begin{array}{l}\text { 2. Forklifts/dollies/ } \\
\text { carts }\end{array}$ & $\mathrm{X}$ & Forklifts (gas and electric) \\
\hline & 3. Railroad & & \\
\hline
\end{tabular}




\begin{tabular}{|c|c|c|c|}
\hline Type of Hazard & Form of Hazard & $\mathbf{X}$ & Remarks \\
\hline & $\begin{array}{l}\text { 4. Obstructions } \\
\text { (collision with) }\end{array}$ & $\mathrm{X}$ & Normal facility obstructions and internal systems \\
\hline & $\begin{array}{l}\text { 5. Crane loads in } \\
\text { motion }\end{array}$ & $\mathrm{X}$ & 20 ton bridge crane \\
\hline & 6. PV blowdown & $\mathrm{X}$ & 109 psig steam system blowdown, ultrafilter back pulse \\
\hline & 7. Other & & \\
\hline \multirow[t]{6}{*}{ D. Kinetic/Rotational } & 1. Centrifuges & & \\
\hline & 2. Motors & $\mathrm{X}$ & \\
\hline & 3. Pumps & $\mathrm{X}$ & \\
\hline & $\begin{array}{l}\text { 4. Cooling tower } \\
\text { fans }\end{array}$ & & \\
\hline & 5. Shop equipment & & \\
\hline & 6. Other & $\mathrm{X}$ & Agitators, HVAC fans, HP air compressor, chiller fans \\
\hline \multirow[t]{10}{*}{ E. Pressure - Volume } & 1. Boilers & $\mathrm{X}$ & Steam boiler - delivers at 109 psig \\
\hline & $\begin{array}{l}\text { 2. Heated surge } \\
\text { tanks }\end{array}$ & $\mathrm{X}$ & HLP22 jacket, UFP 62A heating coil, UFP 2A jacket \\
\hline & 3. Autoclaves & & \\
\hline & $\begin{array}{l}\text { 4. Test loops and } \\
\text { facilities }\end{array}$ & $\mathrm{X}$ & Sampling system \\
\hline & 5. Gas bottles & $\mathrm{X}$ & Propane on forklift \\
\hline & 6. Pressure vessels & & \\
\hline & 7. Stressed members & & \\
\hline & 8. Gas receivers & & \\
\hline & $\begin{array}{l}\text { 9. Negative pressure } \\
\text { collapse }\end{array}$ & $\mathrm{X}$ & Vacuum on pulse jet mixers \\
\hline & 10. Other & $\mathrm{X}$ & $\begin{array}{l}\text { Steam accumulator tanks, compressed air accumulator (100 psi } \\
\text { plant air and booster pump } 500 \mathrm{psi})\end{array}$ \\
\hline \multirow{14}{*}{$\begin{array}{l}\text { F. Mass, Gravity, } \\
\text { Height }\end{array}$} & 1. Human effort & $\mathrm{X}$ & \\
\hline & 2. Stairs & $\mathrm{X}$ & \\
\hline & 3. Lifts and cranes & $\mathrm{X}$ & \\
\hline & 4. Bucket and ladder & $\mathrm{X}$ & \\
\hline & 5. Trucks & $\mathrm{X}$ & Hand trucks \\
\hline & 6. Slings & $\mathrm{X}$ & \\
\hline & 7. Hoists & $\mathrm{X}$ & \\
\hline & 8. Elevators & & \\
\hline & 9. Jacks & $\mathrm{X}$ & \\
\hline & $\begin{array}{l}\text { 10. Scaffolds and } \\
\text { ladders }\end{array}$ & $\mathrm{X}$ & \\
\hline & $\begin{array}{l}\text { 11. Pits and } \\
\text { excavations }\end{array}$ & & \\
\hline & 12. Elevated doors & & \\
\hline & 13. Vessels & $\mathrm{X}$ & \\
\hline & 14. Other & $\mathrm{X}$ & Elevated platform, roll up doors, containment curbs and barriers \\
\hline
\end{tabular}




\begin{tabular}{|c|c|c|c|}
\hline Type of Hazard & Form of Hazard & $\mathbf{X}$ & Remarks \\
\hline \multirow{16}{*}{$\begin{array}{l}\text { G. Flammable } \\
\text { Materials }\end{array}$} & 1. Packing materials & $\mathrm{X}$ & \\
\hline & 2. Rags & $\mathrm{X}$ & \\
\hline & 3. Gasoline & & \\
\hline & 4. Oil & $\mathrm{X}$ & Gear boxes, etc. \\
\hline & 5. Coolant oil & $\mathrm{X}$ & Air compressor, exterior transformer \\
\hline & 6. Paint solvent & & \\
\hline & 7. Diesel fuel & $\mathrm{X}$ & Standard trucks \\
\hline & $\begin{array}{l}\text { 8. Buildings and } \\
\text { contents }\end{array}$ & & \\
\hline & $\begin{array}{l}\text { 9. Trailers and } \\
\text { contents }\end{array}$ & & \\
\hline & 10. Grease & $\mathrm{X}$ & \\
\hline & $\begin{array}{l}\text { 11. Hydrogen } \\
\text { (including battery } \\
\text { banks) }\end{array}$ & & \\
\hline & 12. Nitric acid & $\mathrm{X}$ & 100 gal 2 molar, 50 - 100 gallons outside \\
\hline & 13. Organics & $\mathrm{X}$ & Antifoam agent? \\
\hline & 14. Gases - other & $\mathrm{X}$ & $\begin{array}{l}\text { Natural gas supply, propane tanks on forklifts, maintenance may } \\
\text { bring in acetylene }\end{array}$ \\
\hline & 15. Spray paint & & \\
\hline & 16. Other & $\mathrm{X}$ & $\begin{array}{l}\text { Solvents (acetones, etc.) to clean equipment, exposed insulation on } \\
\text { building/system, cables, plastic piping on pulse jetting, misc paper, } \\
\text { glycol in chillers }\end{array}$ \\
\hline \multirow[t]{6}{*}{ H. Corrosives } & 1. Acids & $\mathrm{X}$ & Nitric, Oxalic (potential replacement for nitric) \\
\hline & 2. Caustics & $\mathrm{X}$ & Sodium hydroxide, Simulant \\
\hline & $\begin{array}{l}\text { 3. "Natural" } \\
\text { chemicals (soil, } \\
\text { air, water) }\end{array}$ & $\mathrm{X}$ & Utilities outside facility \\
\hline & 4. Decon solutions & & \\
\hline & $\begin{array}{l}\text { 5. High temperature } \\
\text { waste }\end{array}$ & $\mathrm{X}$ & $\begin{array}{l}\text { Concentrated solution is heated in tanks }(220 \mathrm{~F}) \text {, Tank UFP T62A, } \\
\mathrm{B} \text { and A during leach }\end{array}$ \\
\hline & 6. Other & $\mathrm{X}$ & Chemical reaction between acids and bases \\
\hline \multirow[t]{9}{*}{ J. Radiation } & 1. Canals & & \\
\hline & 2. Plug storage & & \\
\hline & 3. Storage areas & & \\
\hline & 4. Storage buildings & & \\
\hline & $\begin{array}{l}\text { 5. Radioactive } \\
\text { sources }\end{array}$ & & \\
\hline & 6. Waste and scrap & & \\
\hline & 7. Contamination & & \\
\hline & $\begin{array}{l}\text { 8. Irradiated } \\
\text { experimental and } \\
\text { reactor equipment }\end{array}$ & & \\
\hline & 9. Electric furnace & & \\
\hline
\end{tabular}




\begin{tabular}{|c|c|c|c|}
\hline Type of Hazard & Form of Hazard & $\mathbf{X}$ & Remarks \\
\hline & $\begin{array}{l}\text { 10. Blacklight (e.g., } \\
\text { magniflux) }\end{array}$ & & \\
\hline & 11. Laser & $\mathrm{X}$ & \\
\hline & 12. Medical x-ray & & \\
\hline & $\begin{array}{l}\text { 13. Radiography } \\
\text { equipment and } \\
\text { sources }\end{array}$ & & \\
\hline & 14. Welding & $\mathrm{X}$ & \\
\hline & $\begin{array}{l}\text { 15. Electric arc, other } \\
\text { (high current } \\
\text { circuits) }\end{array}$ & & \\
\hline & 16. Electron beam & & \\
\hline & 17. Equipment noise & $\mathrm{X}$ & Potential for equipment noise \\
\hline & $\begin{array}{l}\text { 18. Ultrasonic } \\
\text { cleaners }\end{array}$ & & \\
\hline & 19. Other & $\mathrm{X}$ & Radar on level indication \\
\hline \multirow[t]{13}{*}{ K. Thermal } & $\begin{array}{l}\text { 1. Bunsen burner/hot } \\
\text { plates }\end{array}$ & $\mathrm{X}$ & Potential for hot plates \\
\hline & $\begin{array}{ll}\text { 2. Electrical } \\
\text { equipment }\end{array}$ & $\mathrm{X}$ & Heat trace \\
\hline & $\begin{array}{l}\text { 3. Furnaces/boilers/ } \\
\text { heater }\end{array}$ & $\mathrm{X}$ & \\
\hline & 4. Steam lines & $\mathrm{X}$ & Steam tracing \\
\hline & 5. Welding torch/arc & $\mathrm{X}$ & \\
\hline & $\begin{array}{l}\text { 6. Diesel units/fire } \\
\text { box/exhaust line }\end{array}$ & & \\
\hline & $\begin{array}{l}\text { 7. Radioactive decay } \\
\text { heat }\end{array}$ & & \\
\hline & $\begin{array}{ll}\text { 8. } & \text { Exposed } \\
\text { components }\end{array}$ & $\mathrm{X}$ & Tops of tanks are not insulated \\
\hline & 9. Power tools & & \\
\hline & 10. Convective & $\mathrm{X}$ & High temperatures in system, condensate lines \\
\hline & 11. Solar & $\mathrm{X}$ & Utilities outside facility \\
\hline & 12. Cryogenic & & \\
\hline & 13. Other & & \\
\hline \multirow{8}{*}{$\begin{array}{l}\text { L. Explosive } \\
\text { Pyrophoric }\end{array}$} & 1. Caps & & \\
\hline & 2. Primer cord & & \\
\hline & 3. Dynamite & & \\
\hline & 4. Scrub chemicals & & \\
\hline & 5. Dusts & & \\
\hline & $\begin{array}{ll}6 . & \text { Hydrogen } \\
& \text { (including battery } \\
\text { banks and water } \\
\text { decomposition) } \\
\end{array}$ & & \\
\hline & 7. Gases, other & & \\
\hline & 8. Nitrates & $\mathrm{X}$ & Sodium nitrate (issue if the material dries out) \\
\hline
\end{tabular}




\begin{tabular}{|c|c|c|c|}
\hline Type of Hazard & Form of Hazard & $\mathbf{X}$ & Remarks \\
\hline & 9. Peroxides & & \\
\hline & 10. Pu and $\mathrm{U}$ metals & & \\
\hline & 11. Sodium & & \\
\hline & 12. Other & & \\
\hline \multirow[t]{12}{*}{ M. Hazardous Material } & 1. Alkali metals & & \\
\hline & 2. Asphyxiants & & \\
\hline & 3. Biologicals & & \\
\hline & 4. Carcinogens & $\mathrm{X}$ & $\begin{array}{l}\text { Potential for nickel contamination in reagents (problem if dried } \\
\text { out) }\end{array}$ \\
\hline & 5. Corrosives & $\mathrm{X}$ & \\
\hline & 6. Asbestos & & \\
\hline & 7. Oxidizers & $\mathrm{X}$ & Sodium permanganate \\
\hline & $\begin{array}{l}\text { 8. Dusts and } \\
\text { particulates }\end{array}$ & $\mathrm{X}$ & $\begin{array}{l}\text { Sodium permanganate is mixed from a dry powder into solution, } \\
\text { dry simulant material (comes in a solution but could dry out) } \\
\text { contains chromium }\end{array}$ \\
\hline & $\begin{array}{l}\text { 9. Beryllium and } \\
\text { compounds }\end{array}$ & & \\
\hline & $\begin{array}{l}\text { 10. Chlorine and } \\
\text { compounds }\end{array}$ & & \\
\hline & 11. Heavy metal & $\mathrm{X}$ & Chromium in simulant \\
\hline & 12. Other & $\mathrm{X}$ & Boiler feed water treatment chemicals (TBD) \\
\hline \multirow[t]{10}{*}{ N. Natural Phenomena } & 1. Earthquake & $\mathrm{X}$ & \\
\hline & 2. Flood & & \\
\hline & 3. Lightning & $\mathrm{X}$ & Utilities outside facility \\
\hline & 4. Rain & $\mathrm{X}$ & Utilities outside facility \\
\hline & $\begin{array}{l}\text { 5. Snow, freezing } \\
\text { weather }\end{array}$ & $\mathrm{X}$ & Outside supply lines \\
\hline & 6. Straight wind & $\mathrm{X}$ & \\
\hline & 7. Dust devil & $\mathrm{X}$ & \\
\hline & 8. Tornado & & \\
\hline & 9. Ashfall & $\mathrm{X}$ & Utilities outside facility \\
\hline & 10. High temperatures & $\mathrm{X}$ & Results in high temperatures in facility \\
\hline \multirow[t]{5}{*}{ O. External Events } & 1. Explosion & & \\
\hline & 2. Fire & $\mathrm{X}$ & Range fire \\
\hline & $\begin{array}{l}\text { 3. Events at other } \\
\text { sites }\end{array}$ & $\mathrm{X}$ & PDL East facility event from hydrogen, etc. \\
\hline & 4. Loss of power & $\mathrm{X}$ & \\
\hline & 5. Other & $\mathrm{X}$ & Loss of outside utilities (natural gas, water, sewer) \\
\hline \multirow{5}{*}{$\begin{array}{l}\text { P. Vehicles in Motion } \\
\text { (external to facility) }\end{array}$} & 1. Airplane & & \\
\hline & 2. Helicopter & & \\
\hline & 3. Train & & \\
\hline & 4. Truck/bus/car & $\mathrm{X}$ & Traffic, truck deliveries \\
\hline & 5. Other & & \\
\hline
\end{tabular}


Appendix E

Hazard Analysis Worksheets 
Table E.1. Hazard Analysis Worksheets

\begin{tabular}{|c|c|c|c|c|c|c|c|c|c|c|c|}
\hline $\begin{array}{l}\text { Scenario } \\
\text { Number }\end{array}$ & Node & $\begin{array}{l}\text { Hazardous } \\
\text { Condition }\end{array}$ & Cause & $\mathbf{L}$ & C & $\begin{array}{c}\text { Risk } \\
\text { Score }\end{array}$ & Potential Safeguard & $\begin{array}{c}\text { Credited } \\
\text { Safeguards }\end{array}$ & $\begin{array}{l}\text { Comments/ } \\
\text { Assumptions }\end{array}$ & E & $\$$ \\
\hline PEP-30-1-01 & $1 \mathrm{a}$ & Operational upset. & $\begin{array}{l}\text { No flow into the receipt } \\
\text { tank due to closed } \\
\text { isolation valve. Potential } \\
\text { to damage pump. }\end{array}$ & & 0 & $\mathrm{n} / \mathrm{a}$ & & $\mathrm{n} / \mathrm{a}$ & $\begin{array}{l}\text { The pump is being } \\
\text { supplied by PNNL. } \\
\text { Look at during } \\
\text { integration. This is } \\
\text { not a prototypic } \\
\text { pump. }\end{array}$ & E0 & 0 \\
\hline PEP-30-1-02 & 1a & $\begin{array}{l}\text { Spill of simulant } \\
\text { (inside or outside } \\
\text { facility). }\end{array}$ & $\begin{array}{l}\text { No flow into the receipt } \\
\text { tank due to leak at the } \\
\text { connection to the simulant } \\
\text { addition flex hose. }\end{array}$ & 1 & 4 & 5 & $\begin{array}{l}\text { Facility secondary containment } \\
\text { Operating procedures (valve lineup) } \\
\text { PPE } \\
\text { Personnel training } \\
\text { Barrier around delivery truck } \\
\text { Receipt tank level detection }\end{array}$ & $\begin{array}{l}\text { PPE } \\
\text { Operating procedures } \\
\text { Facility secondary } \\
\text { containment } \\
\text { Receipt tank level } \\
\text { detection }\end{array}$ & $\begin{array}{l}\text { Potential for caustic } \\
\text { burn/eye damage } \\
\mathrm{pH} \sim 12 \text {. }\end{array}$ & E2 & 1 \\
\hline PEP-30-1-03 & $1 \mathrm{a}$ & $\begin{array}{l}\text { Spill of simulant, } \\
\text { sodium hydroxide, } \\
\text { material from } \\
\text { other tanks or } \\
\text { water. }\end{array}$ & $\begin{array}{l}\text { No flow into the receipt } \\
\text { tank due to leak in } \\
\text { simulant addition or } \\
\text { transfer piping (flanges, } \\
\text { etc.). }\end{array}$ & 0 & 4 & 4 & \begin{tabular}{|l|} 
Leak test at installation \\
Facility secondary containment \\
Operating procedures (valve lineup) \\
PPE \\
Personnel training \\
Receipt tank level detection \\
\end{tabular} & $\begin{array}{l}\text { PPE } \\
\text { Operating procedures } \\
\text { Facility secondary } \\
\text { containment } \\
\text { Receipt tank level } \\
\text { detection } \\
\end{array}$ & $\begin{array}{l}\text { Potential for caustic } \\
\text { burn/eye damage } \\
\mathrm{pH} 14 . \\
\text { Leak is inside facility. }\end{array}$ & E0 & 1 \\
\hline PEP-30-1-04 & $1 \mathrm{a}$ & Operational upset. & $\begin{array}{l}\text { No flow out of receipt } \\
\text { tank due to pump not } \\
\text { operating. }\end{array}$ & & 0 & $\mathrm{n} / \mathrm{a}$ & & $\mathrm{n} / \mathrm{a}$ & & E0 & 0 \\
\hline PEP-30-1-05 & $1 \mathrm{a}$ & Operational upset. & $\begin{array}{l}\text { No flow out of receipt } \\
\text { tank due to line plugging. }\end{array}$ & & 0 & $\mathrm{n} / \mathrm{a}$ & & $\mathrm{n} / \mathrm{a}$ & & E0 & 0 \\
\hline PEP-30-1-06 & 1a & $\begin{array}{l}\text { Spill of simulant, } \\
\text { sodium hydroxide, } \\
\text { material from } \\
\text { other tanks or } \\
\text { water. }\end{array}$ & $\begin{array}{l}\text { Structural integrity of } \\
\text { receipt tank degraded } \\
\text { resulting in leak. }\end{array}$ & -3 & 4 & 1 & Design of tanks to code & $\begin{array}{l}\text { Design of receipt } \\
\text { tanks to code }\end{array}$ & $\begin{array}{l}\text { Spill to floor of } \\
\text { facility. } \\
\text { Potential for caustic } \\
\text { burn/eye damage } \\
\text { pH 14. } \\
\text { Likelihood assigned } \\
\text { based on tank to code. } \\
304 \text { SST is listed as } \\
\text { having excellent } \\
\text { chemical } \\
\text { compatibility to nitric } \\
\text { acid, and good } \\
\text { compatibility with } \\
\text { NaOH, and oxalic } \\
\text { acid. }\end{array}$ & E0 & SD \\
\hline
\end{tabular}


Table E.1. Hazard Analysis Worksheets

\begin{tabular}{|c|c|c|c|c|c|c|c|c|c|c|c|}
\hline $\begin{array}{l}\text { Scenario } \\
\text { Number } \\
\end{array}$ & Node & $\begin{array}{c}\text { Hazardous } \\
\text { Condition }\end{array}$ & Cause & $\mathbf{L}$ & C & $\begin{array}{l}\text { Risk } \\
\text { Score }\end{array}$ & Potential Safeguard & $\begin{array}{l}\text { Credited } \\
\text { Safeguards }\end{array}$ & $\begin{array}{c}\text { Comments/ } \\
\text { Assumptions }\end{array}$ & $\mathbf{E}$ & $\$$ \\
\hline PEP-30-1-07 & $1 \mathrm{a}$ & \begin{tabular}{|l|} 
Spill of simulant, \\
sodium hydroxide, \\
material, or water \\
from other tanks.
\end{tabular} & $\begin{array}{l}\text { No flow out of the } \\
\text { Receipt tank due to leak } \\
\text { in transfer piping/pumps. }\end{array}$ & 0 & 4 & 4 & $\begin{array}{l}\text { Leak test at installation } \\
\text { Facility secondary containment } \\
\text { Operating procedures (valve lineup) } \\
\text { PPE } \\
\text { Personnel training } \\
\text { Receipt tank level detection } \\
\text { Pump design features }\end{array}$ & \begin{tabular}{|l|} 
PPE \\
Operating procedures \\
Facility secondary \\
containment \\
Receipt tank level \\
detection
\end{tabular} & $\begin{array}{l}\text { Spill to floor of } \\
\text { facility. } \\
\text { Potential for caustic } \\
\text { burn/eye damage } \\
\text { pH 14. }\end{array}$ & E0 & $\mathrm{SD}$ \\
\hline PEP-30-1-08 & $1 \mathrm{a}$ & Operational upset. & $\begin{array}{l}\text { Lower-than-intended } \\
\text { receipt tank volume. }\end{array}$ & & 0 & $\mathrm{n} / \mathrm{a}$ & & $\mathrm{n} / \mathrm{a}$ & & E0 & 0 \\
\hline PEP-30-1-09 & $1 \mathrm{a}$ & $\begin{array}{l}\text { No issue } \\
\text { identified. }\end{array}$ & $\begin{array}{l}\text { Filling receipt tank too } \\
\text { fast. }\end{array}$ & & 0 & $\mathrm{n} / \mathrm{a}$ & & $\mathrm{n} / \mathrm{a}$ & $\begin{array}{l}\text { Ventilation system is } \\
\text { designed to } \\
\text { accommodate for any } \\
\text { excess aerosols } \\
\text { generated during this } \\
\text { event. }\end{array}$ & E0 & 0 \\
\hline PEP-30-1-10 & $1 \mathrm{a}$ & Operational upset. & $\begin{array}{l}\text { Too much material added } \\
\text { to the receipt tank } \\
\text { resulting in boiling in } \\
\text { T22. Excess aerosols } \\
\text { enter ventilation system. }\end{array}$ & & 0 & $\mathrm{n} / \mathrm{a}$ & & $\mathrm{n} / \mathrm{a}$ & $\begin{array}{l}\text { Aerosols generated } \\
\text { during boiling will } \\
\text { not exceed } \\
\text { environment permit } \\
\text { limits. }\end{array}$ & E0 & 0 \\
\hline PEP-30-1-11 & $1 \mathrm{a}$ & Operation upset. & $\begin{array}{l}\text { Too much material added } \\
\text { to the receipt tank } \\
\text { resulting in overflow of } \\
\text { tank with valves open } \\
\text { results in overflow of tank } \\
\text { cascading to other vessels. }\end{array}$ & & 0 & $\mathrm{n} / \mathrm{a}$ & & $\mathrm{n} / \mathrm{a}$ & & E0 & 0 \\
\hline PEP-30-1-12 & $1 \mathrm{a}$ & \begin{tabular}{|l|} 
Overflow of tank \\
contents to \\
ventilation system, \\
damaging fans, \\
exchanger and \\
blower. Boil off \\
liquids forming \\
solids in vent \\
lines. \\
\end{tabular} & $\begin{array}{l}\text { Too much material added } \\
\text { to the receipt tank } \\
\text { resulting in overflow of } \\
\text { tank with valves closed. }\end{array}$ & 0 & 0 & 2 & $\begin{array}{l}\text { Level detection, alarms and automatic } \\
\text { closure of inlet valves } \\
\text { Flow totalizer with automatic closure } \\
\text { of inlet valve } \\
\text { Operating procedures }\end{array}$ & $\begin{array}{l}\text { Receipt tank level } \\
\text { detection and alarms }\end{array}$ & & E2 & 2 \\
\hline PEP-30-1-13 & $1 \mathrm{a}$ & \begin{tabular}{|l|} 
Incomplete/out of \\
spec batch of \\
material. Lost \\
time for test.
\end{tabular} & $\begin{array}{l}\text { Too much material added } \\
\text { to the receipt tank. High } \\
\text { level in tank. }\end{array}$ & 1 & 0 & 3 & $\begin{array}{l}\text { Level detection } \\
\text { Flow totalizer } \\
\text { Operating procedures }\end{array}$ & $\begin{array}{l}\text { Receipt tank level } \\
\text { detection and alarms }\end{array}$ & & E0 & 2 \\
\hline PEP-30-1-14 & $1 \mathrm{a}$ & Operational upset. & $\begin{array}{l}\text { Too much caustic added; } \\
\text { causes formation of } \\
\text { oxalates in receipt tank. }\end{array}$ & & 0 & $\mathrm{n} / \mathrm{a}$ & & $\mathrm{n} / \mathrm{a}$ & & E0 & 0 \\
\hline
\end{tabular}


Table E.1. Hazard Analysis Worksheets

\begin{tabular}{|c|c|c|c|c|c|c|c|c|c|c|c|}
\hline $\begin{array}{l}\text { Scenario } \\
\text { Number } \\
\end{array}$ & Node & $\begin{array}{c}\text { Hazardous } \\
\text { Condition }\end{array}$ & Cause & $\mathbf{L}$ & C & $\begin{array}{l}\text { Risk } \\
\text { Score } \\
\end{array}$ & Potential Safeguard & $\begin{array}{l}\text { Credited } \\
\text { Safeguards }\end{array}$ & $\begin{array}{c}\text { Comments/ } \\
\text { Assumptions }\end{array}$ & $\mathbf{E}$ & $\$$ \\
\hline PEP-30-1-15 & $1 \mathrm{a}$ & $\begin{array}{l}\text { Incomplete/out of } \\
\text { spec batch of } \\
\text { material. Lost } \\
\text { time for test. }\end{array}$ & $\begin{array}{l}\text { Too little caustic added to } \\
\text { receipt tank resulting in } \\
\text { Tank UFP-VSL-T01A/B } \\
\text { contents too low in } \\
\text { caustic. }\end{array}$ & 1 & 0 & 3 & $\begin{array}{l}\text { Level detection } \\
\text { Flow totalizer } \\
\text { Operating procedures }\end{array}$ & $\begin{array}{l}\text { Receipt tank level } \\
\text { detection }\end{array}$ & & E0 & 2 \\
\hline PEP-30-1-16 & $1 \mathrm{a}$ & $\begin{array}{l}\text { Incomplete/out of } \\
\text { spec batch of } \\
\text { material. Lost } \\
\text { time for test. }\end{array}$ & $\begin{array}{l}\text { Delivery of "out of spec" } \\
\text { caustic or simulant results } \\
\text { in wrong batch specs. }\end{array}$ & 1 & 0 & 3 & $\begin{array}{l}\text { Require certification from vendor with } \\
\text { each batch. }\end{array}$ & Operating procedures & & E0 & 2 \\
\hline PEP-30-1-17 & $1 \mathrm{a}$ & $\begin{array}{l}\text { Incomplete/out of } \\
\text { spec batch of } \\
\text { material. Lost } \\
\text { time for test. }\end{array}$ & $\begin{array}{l}\text { Incorrect valve lineup } \\
\text { between receipt tanks } \\
\text { creates a misbatching. }\end{array}$ & 1 & 0 & 3 & \begin{tabular}{|l|} 
Flow totalizer \\
Operating procedures
\end{tabular} & Operating procedures & & E0 & 2 \\
\hline PEP-30-1-18 & $1 \mathrm{a}$ & \begin{tabular}{|l|} 
Overflow of \\
receipt tank \\
contents to \\
ventilation system, \\
damaging fans, \\
exchanger, and \\
blower. Boil off \\
liquids forming \\
solids in vent \\
lines.
\end{tabular} & $\begin{array}{l}\text { Backflow of material } \\
\text { from UFP-VSL-Y01A/B } \\
\text { to receipt tank caused by } \\
\text { transfer of material into } \\
\text { A/B overflowing back. }\end{array}$ & 1 & 0 & 3 & $\begin{array}{l}\text { Level detection, alarms and automatic } \\
\text { closure of inlet valves } \\
\text { Flow totalizer with automatic closure } \\
\text { of inlet valve } \\
\text { Operating procedures }\end{array}$ & $\begin{array}{l}\text { Receipt tank level } \\
\text { detection and alarms }\end{array}$ & & E2 & 2 \\
\hline PEP-30-1-19 & 1a & Operational upset. & $\begin{array}{l}\text { Low pressure in receipt } \\
\text { tank due to plugging of } \\
\text { ventilation (closure of } \\
\text { damper) during transfer } \\
\text { out. }\end{array}$ & & 0 & $\mathrm{n} / \mathrm{a}$ & Design of tanks to 7.5 psid external & $\mathrm{n} / \mathrm{a}$ & $\begin{array}{l}\text { System is designed } \\
\text { such that the pumps } \\
\text { cannot draw suction } \\
\text { enough to damage } \\
\text { tank. }\end{array}$ & E0 & 0 \\
\hline PEP-30-1-21 & $1 \mathrm{a}$ & $\begin{array}{l}\text { Failure of tank and } \\
\text { steam jacket and } \\
\text { spill of tank } \\
\text { contents. }\end{array}$ & $\begin{array}{l}\text { High pressure in receipt } \\
\text { tank due to ventilation } \\
\text { blocked (closure of } \\
\text { damper) and overfilling of } \\
\text { tank. }\end{array}$ & 1 & 5 & 6 & $\begin{array}{l}\text { Pressure relief valve } \\
\text { Local pressure indicator for tank } \\
\text { Operating procedures } \\
\text { Pressure transmitter with } \mathrm{AH} \& \mathrm{AHH} \\
\text { on tanks }\end{array}$ & $\begin{array}{l}\text { PSV on receipt tank } \\
\text { Stop or bore through } \\
\text { on the damper to } \\
\text { prevent full closure }\end{array}$ & $\begin{array}{l}\text { Spill to floor of } \\
\text { facility. }\end{array}$ & E0 & SD \\
\hline PEP-30-1-22 & $1 \mathrm{a}$ & \begin{tabular}{|l|} 
Failure to tank and \\
steam jacket and \\
spill of tank \\
contents.
\end{tabular} & $\begin{array}{l}\text { High pressure in receipt } \\
\text { tank due to ventilation } \\
\text { blocked (closure of } \\
\text { damper) and overheat the } \\
\text { tank due to temperature } \\
\text { control system failure. }\end{array}$ & -1 & 5 & 4 & $\begin{array}{l}\text { Pressure relief valve on receipt tank } \\
\text { Local pressure indicator for tank } \\
\text { Temperature controls } \\
\text { Operating procedures } \\
\text { Pressure transmitter with AH \& AHH } \\
\text { on tanks } \\
\text { Stop or bore through on the damper to } \\
\text { prevent full closure. }\end{array}$ & $\begin{array}{l}\text { PSV on receipt tank; } \\
\text { Stop or bore through } \\
\text { on the damper to } \\
\text { prevent full closure. }\end{array}$ & $\begin{array}{l}\text { Spill to floor of } \\
\text { facility. Steam } \\
\text { release is potential } \\
\text { when heating. }\end{array}$ & E0 & SD \\
\hline
\end{tabular}


Table E.1. Hazard Analysis Worksheets

\begin{tabular}{|c|c|c|c|c|c|c|c|c|c|c|c|}
\hline $\begin{array}{l}\text { Scenario } \\
\text { Number }\end{array}$ & Node & $\begin{array}{c}\text { Hazardous } \\
\text { Condition }\end{array}$ & Cause & $\mathbf{L}$ & C & $\begin{array}{c}\text { Risk } \\
\text { Score }\end{array}$ & Potential Safeguard & $\begin{array}{l}\text { Credited } \\
\text { Safeguards }\end{array}$ & $\begin{array}{l}\text { Comments/ } \\
\text { Assumptions }\end{array}$ & E & $\$$ \\
\hline PEP-30-1-23 & $1 \mathrm{a}$ & Operational upset. & $\begin{array}{l}\text { Lost heat trace on caustic } \\
\text { addition line. Plug line } \\
\text { with material - lose } \\
\text { caustic to receipt tanks. }\end{array}$ & & 0 & $\mathrm{n} / \mathrm{a}$ & & $\mathrm{n} / \mathrm{a}$ & & E0 & 0 \\
\hline PEP-30-1-24 & 1a & Operational upset. & $\begin{array}{l}\text { High temperature in } \\
\text { receipt tank due to } \\
\text { overheating. Boil } \\
\text { contents of tank, creates } \\
\text { excess aerosols. Excess } \\
\text { aerosols enter ventilation } \\
\text { system. }\end{array}$ & & 0 & $\mathrm{n} / \mathrm{a}$ & Temperature indicators & $\mathrm{n} / \mathrm{a}$ & $\begin{array}{l}\text { The aerosols } \\
\text { generated during } \\
\text { boiling will not } \\
\text { exceed environmental } \\
\text { permit limits. }\end{array}$ & E0 & 0 \\
\hline PEP-30-1-25 & 1a & Operational upset. & $\begin{array}{l}\text { High temperature in } \\
\text { receipt tank dries out } \\
\text { material. }\end{array}$ & & 0 & $\mathrm{n} / \mathrm{a}$ & Temperature indicators & $\mathrm{n} / \mathrm{a}$ & & E0 & 0 \\
\hline PEP-30-1-26 & $1 \mathrm{a}$ & Operational upset. & $\begin{array}{l}\text { Agitator in receipt tank } \\
\text { does not operate or poor } \\
\text { mixing due to VFD too } \\
\text { slow. }\end{array}$ & & 0 & $\mathrm{n} / \mathrm{a}$ & & $\mathrm{n} / \mathrm{a}$ & & E0 & 0 \\
\hline PEP-30-1-27 & $1 \mathrm{a}$ & $\begin{array}{l}\text { Carryover of } \\
\text { material into the } \\
\text { ventilation system. }\end{array}$ & $\begin{array}{l}\text { Agitation causes foaming } \\
\text { in the receipt tank. }\end{array}$ & 1 & 0 & 3 & $\begin{array}{l}\text { Sight glass } \\
\text { Operating procedures } \\
\text { Anti-foaming agent }\end{array}$ & None identified & & E1 & 2 \\
\hline PEP-30-1-28 & $1 \mathrm{a}$ & Operational upset. & $\begin{array}{l}\text { Prolonged settling in } \\
\text { receipt tank causes } \\
\text { accumulation of solids in } \\
\text { tank results in difficulty } \\
\text { moving/mixing of } \\
\text { material. }\end{array}$ & & 0 & $\mathrm{n} / \mathrm{a}$ & Operating procedures & $\mathrm{n} / \mathrm{a}$ & & E0 & 0 \\
\hline PEP-30-1-29 & $1 \mathrm{a}$ & Operational upset. & $\begin{array}{l}\text { Low temperature in the } \\
\text { steam jacket. Cannot heat } \\
\text { receipt tank. }\end{array}$ & & 0 & $\mathrm{n} / \mathrm{a}$ & & $\mathrm{n} / \mathrm{a}$ & & E0 & 0 \\
\hline PEP-30-1-30 & $1 \mathrm{a}$ & $\begin{array}{l}\text { Small release of } \\
\text { steam to the } \\
\text { facility. }\end{array}$ & $\begin{array}{l}\text { Structural failure of the } \\
\text { steam jacket on receipt } \\
\text { tank. Small leak of steam. }\end{array}$ & -1 & 2 & 1 & Pressure relief on the steam jacket & $\begin{array}{l}\text { PSV on the steam } \\
\text { jacket }\end{array}$ & & E0 & 1 \\
\hline PEP-30-1-31 & $1 \mathrm{a}$ & $\begin{array}{l}\text { Large release of } \\
\text { steam to the } \\
\text { facility. }\end{array}$ & $\begin{array}{l}\text { Catastrophic failure of the } \\
\text { steam jacket on receipt } \\
\text { tank results in large } \\
\text { release of steam. }\end{array}$ & -3 & 5 & 2 & $\begin{array}{l}\text { Design of steam jacket to code } \\
\text { Pressure relief on the steam jacket }\end{array}$ & \begin{tabular}{|l} 
PSV on the steam \\
jacket
\end{tabular} & & E0 & 3 \\
\hline
\end{tabular}


Table E.1. Hazard Analysis Worksheets

\begin{tabular}{|c|c|c|c|c|c|c|c|c|c|c|c|}
\hline $\begin{array}{l}\text { Scenario } \\
\text { Number }\end{array}$ & Node & $\begin{array}{c}\text { Hazardous } \\
\text { Condition }\end{array}$ & Cause & $\mathbf{L}$ & C & $\begin{array}{l}\text { Risk } \\
\text { Score }\end{array}$ & Potential Safeguard & $\begin{array}{c}\text { Credited } \\
\text { Safeguards }\end{array}$ & $\begin{array}{c}\text { Comments/ } \\
\text { Assumptions }\end{array}$ & $\mathbf{E}$ & $\$$ \\
\hline PEP-30-1-32 & $1 \mathrm{a}$ & $\begin{array}{l}\text { Collapse of steam } \\
\text { jacket. }\end{array}$ & $\begin{array}{l}\text { Steam present in the } \\
\text { steam jacket on the receipt } \\
\text { tank. Block off jacket, } \\
\text { add cold water to refill. } \\
\text { Vacuum pulled collapsing } \\
\text { jacket. }\end{array}$ & -1 & 0 & 2 & $\begin{array}{l}\text { Vacuum breakers on jacket } \\
\text { Operating procedures }\end{array}$ & $\begin{array}{l}\text { Vacuum breaker on } \\
\text { steam jacket }\end{array}$ & & E0 & 3 \\
\hline PEP-90-1-01 & 1a & $\begin{array}{l}\text { Small release of } \\
\text { steam to the } \\
\text { facility. }\end{array}$ & $\begin{array}{l}\text { Failure of flex hose } \\
\text { connection from steam } \\
\text { jacket to steam supply } \\
\text { system (steam line or } \\
\text { traps). Release steam to } \\
\text { the facility. }\end{array}$ & -1 & 2 & 1 & & None identified & & E0 & 1 \\
\hline PEP-30-2-01 & 2 & Operational upset. & $\begin{array}{l}\text { No flow into the feed } \\
\text { preparation tank due to } \\
\text { closed isolation valve. }\end{array}$ & & 0 & $\mathrm{n} / \mathrm{a}$ & & $\mathrm{n} / \mathrm{a}$ & & E0 & 0 \\
\hline PEP-30-2-02 & 2 & $\begin{array}{l}\text { Spill of simulant, } \\
\text { sodium hydroxide, } \\
\text { material, or } \\
\text { inhibited water } \\
\text { from other tanks. }\end{array}$ & $\begin{array}{l}\text { No flow into the feed } \\
\text { preparation tank due to } \\
\text { leak in chemical addition } \\
\text { or transfer piping (flanges, } \\
\text { etc.). }\end{array}$ & 0 & 4 & 4 & $\begin{array}{l}\text { Leak test at installation } \\
\text { Facility secondary containment } \\
\text { Operating procedures } \\
\text { PPE } \\
\text { Personnel training } \\
\text { Feed preparation tank level detection } \\
\text { (thermocouples, bubblers, and laser) } \\
\text { Flow totalizers on major chemical } \\
\text { additions } \\
\text { Flow meter on transfer line }\end{array}$ & $\begin{array}{l}\text { PPE } \\
\text { Operating procedures } \\
\text { Facility secondary } \\
\text { containment } \\
\text { Feed Preparation } \\
\text { tank level detection }\end{array}$ & $\begin{array}{l}\text { Potential for caustic } \\
\text { burn/eye damage } \\
\text { pH 14. } \\
\text { Leak is inside facility. }\end{array}$ & E0 & 1 \\
\hline $\mid \overline{\text { PEP-30-2-03 }}$ & 2 & Operational upset. & $\begin{array}{l}\text { No flow into the feed } \\
\text { preparation tank due to } \\
\text { pump failure or plugging } \\
\text { of chemical addition or } \\
\text { transfer piping (flanges, } \\
\text { etc.). }\end{array}$ & & 0 & $\mathrm{n} / \mathrm{a}$ & & $\mathrm{n} / \mathrm{a}$ & & E0 & 0 \\
\hline PEP-30-2-04 & 2 & Operational upset. & $\begin{array}{l}\text { No flow out of feed } \\
\text { preparation tank due to } \\
\text { pump not operating. }\end{array}$ & & 0 & $\mathrm{n} / \mathrm{a}$ & & $\mathrm{n} / \mathrm{a}$ & & E0 & 0 \\
\hline $\mid \overline{\text { PEP-30-2-05 }}$ & 2 & Operational upset. & $\begin{array}{l}\text { No flow out of feed } \\
\text { preparation tank due to } \\
\text { line plugging. }\end{array}$ & & 0 & $\mathrm{n} / \mathrm{a}$ & & $\mathrm{n} / \mathrm{a}$ & & E0 & 0 \\
\hline PEP-30-2-06 & 2 & $\begin{array}{l}\text { Spill of tank } \\
\text { contents. }\end{array}$ & $\begin{array}{l}\text { Structural integrity of feed } \\
\text { preparation tank degraded } \\
\text { resulting in leak. }\end{array}$ & -3 & 4 & 1 & $\begin{array}{l}\text { Design of feed preparation tanks to } \\
\text { code }\end{array}$ & $\begin{array}{l}\text { Design of feed } \\
\text { preparation tanks to } \\
\text { code }\end{array}$ & $\begin{array}{l}\text { Spill to floor of } \\
\text { facility. } \\
\text { Potential for caustic } \\
\text { burn/eye damage. } \\
\text { pH 14. }\end{array}$ & E0 & 3 \\
\hline
\end{tabular}


Table E.1. Hazard Analysis Worksheets

\begin{tabular}{|c|c|c|c|c|c|c|c|c|c|c|c|}
\hline $\begin{array}{l}\text { Scenario } \\
\text { Number }\end{array}$ & Node & $\begin{array}{l}\text { Hazardous } \\
\text { Condition }\end{array}$ & Cause & $\mathbf{L}$ & C & $\begin{array}{c}\text { Risk } \\
\text { Score }\end{array}$ & Potential Safeguard & $\begin{array}{c}\text { Credited } \\
\text { Safeguards }\end{array}$ & $\begin{array}{l}\text { Comments/ } \\
\text { Assumptions }\end{array}$ & $\mathbf{E}$ & $\$$ \\
\hline & & & & & & & & & $\begin{array}{l}\text { Likelihood assigned } \\
\text { based on tank to code. } \\
304 \text { SST is listed as } \\
\text { having excellent } \\
\text { chemical } \\
\text { compatibility to nitric } \\
\text { acid, and good } \\
\text { compatibility with } \\
\text { NaOH and oxalic } \\
\text { acid. }\end{array}$ & & \\
\hline PEP-30-2-07 & 2 & $\begin{array}{l}\text { Spill of tank } \\
\text { contents. }\end{array}$ & $\begin{array}{l}\text { No flow out of the feed } \\
\text { preparation tank due to } \\
\text { leak in transfer } \\
\text { piping/pumps. }\end{array}$ & 0 & 4 & 4 & $\begin{array}{l}\text { Leak test at installation } \\
\text { Facility secondary containment } \\
\text { Operating procedures } \\
\text { PPE } \\
\text { Personnel training } \\
\text { Feed preparation and ultrafiltration } \\
\text { feed tank level detection } \\
\text { (thermocouples, bubblers, and laser) } \\
\text { Flow meter on transfer line }\end{array}$ & \begin{tabular}{|l|} 
PPE \\
Operating procedures \\
Facility secondary \\
containment \\
Feed Preparation \\
tank level detection
\end{tabular} & $\begin{array}{l}\text { Spill to floor of } \\
\text { facility. } \\
\text { Potential for caustic } \\
\text { burn/eye damage } \\
\text { pH 14. }\end{array}$ & E0 & SD \\
\hline PEP-30-2-08 & 2 & $\begin{array}{l}\text { Carryover of } \\
\text { material to the } \\
\text { ventilation system. }\end{array}$ & $\begin{array}{l}\text { Lower-than-intended feed } \\
\text { preparation tank volume. } \\
\text { Level in tank below level } \\
\text { of the PJMs results in } \\
\text { overblow. Material in the } \\
\text { vent line. }\end{array}$ & 1 & 0 & 3 & $\begin{array}{l}\text { PLC } \\
\text { PJM Level Detector } \\
\text { PJM Pressure Detector } \\
\text { PJM Rack Air Supply Valve }\end{array}$ & $\begin{array}{l}\text { PLC } \\
\text { PJM Level Detector } \\
\text { PJM Pressure } \\
\text { Detector } \\
\text { PJM Rack Air } \\
\text { Supply Valve } \\
\text { Feed Preparation } \\
\text { tank level detection }\end{array}$ & $\begin{array}{l}\text { Revised at } 90 \% \text { to } \\
\text { reflect PJM overblow } \\
\text { controls }\end{array}$ & E1 & 2 \\
\hline PEP-30-2-09 & 2 & $\begin{array}{l}\text { No issue } \\
\text { identified. }\end{array}$ & $\begin{array}{l}\text { Filling feed preparation } \\
\text { tank too fast. }\end{array}$ & & 0 & $\mathrm{n} / \mathrm{a}$ & & $\mathrm{n} / \mathrm{a}$ & $\begin{array}{l}\text { Ventilation system is } \\
\text { designed to } \\
\text { accommodate for any } \\
\text { excess aerosols } \\
\text { generated during this } \\
\text { event. }\end{array}$ & E0 & 0 \\
\hline PEP-30-2-10 & 2 & Operational upset. & $\begin{array}{l}\text { Too much caustic material } \\
\text { added into the feed } \\
\text { preparation tank results in } \\
\text { boiling. Excess aerosols } \\
\text { enter ventilation system. }\end{array}$ & & 0 & $\mathrm{n} / \mathrm{a}$ & & $\mathrm{n} / \mathrm{a}$ & $\begin{array}{l}\text { Aerosols generated } \\
\text { during boiling will } \\
\text { not exceed } \\
\text { environment permit } \\
\text { limits. }\end{array}$ & E0 & 0 \\
\hline
\end{tabular}


Table E.1. Hazard Analysis Worksheets

\begin{tabular}{|c|c|c|c|c|c|c|c|c|c|c|c|}
\hline $\begin{array}{l}\text { Scenario } \\
\text { Number }\end{array}$ & Node & $\begin{array}{c}\text { Hazardous } \\
\text { Condition }\end{array}$ & Cause & $\mathbf{L}$ & C & $\begin{array}{l}\text { Risk } \\
\text { Score }\end{array}$ & Potential Safeguard & $\begin{array}{l}\text { Credited } \\
\text { Safeguards }\end{array}$ & $\begin{array}{c}\text { Comments/ } \\
\text { Assumptions }\end{array}$ & $\mathbf{E}$ & $\$$ \\
\hline PEP-30-2-11 & 2 & $\begin{array}{l}\text { Spill tank contents } \\
\text { to floor. }\end{array}$ & $\begin{array}{l}\text { Too much material added } \\
\text { to the feed preparation } \\
\text { tank, resulting in overflow } \\
\text { of tank to facility floor. }\end{array}$ & 0 & 4 & 4 & $\begin{array}{l}\text { Facility secondary containment } \\
\text { Operating procedures } \\
\text { PPE } \\
\text { Personnel training } \\
\text { Feed preparation tank level detection } \\
\text { (thermocouples, bubblers, and laser) } \\
\text { Flow totalizers on major chemical } \\
\text { additions } \\
\text { Flow meter on transfer line }\end{array}$ & \begin{tabular}{|l|} 
PPE \\
Operating procedures \\
Facility secondary \\
containment \\
Feed Preparation \\
tank level detection \\
and alarms
\end{tabular} & $\begin{array}{l}\text { Spill to floor of } \\
\text { facility. } \\
\text { Potential for caustic } \\
\text { burn/eye damage } \\
\text { pH 14. }\end{array}$ & E0 & $\mathrm{SD}$ \\
\hline PEP-30-2-12 & 2 & $\begin{array}{l}\text { Incomplete/out of } \\
\text { spec batch of } \\
\text { material. Lost } \\
\text { time for test. }\end{array}$ & $\begin{array}{l}\text { Too much material added } \\
\text { to the feed preparation } \\
\text { tank. High level in tank. }\end{array}$ & 1 & 0 & 3 & $\begin{array}{l}\text { Operating procedures } \\
\text { Feed preparation tank level detection } \\
\text { (thermocouples, bubblers, and laser) } \\
\text { Flow totalizers on major chemical } \\
\text { additions } \\
\text { Flow meter on transfer line }\end{array}$ & $\begin{array}{l}\text { Feed Preparation } \\
\text { tank level detection } \\
\text { and alarms }\end{array}$ & & E0 & 2 \\
\hline PEP-30-2-13 & 2 & Operational upset. & $\begin{array}{l}\text { Too much caustic added } \\
\text { to the feed preparation } \\
\text { tank causes formation of } \\
\text { oxalates in tank. } \\
\end{array}$ & & 0 & $\mathrm{n} / \mathrm{a}$ & & $\mathrm{n} / \mathrm{a}$ & & E0 & 0 \\
\hline PEP-30-2-14 & 2 & $\begin{array}{l}\text { Incomplete/“out of } \\
\text { spec" batch of } \\
\text { material. Lost } \\
\text { time for test. }\end{array}$ & $\begin{array}{l}\text { Too little caustic added to } \\
\text { the feed preparation tank } \\
\text { results in Tank UFP-VSL- } \\
\text { T02A caustic } \\
\text { concentration being too } \\
\text { low. }\end{array}$ & 1 & 0 & 3 & $\begin{array}{l}\text { Operating procedures } \\
\text { Feed preparation tank level detection } \\
\text { (thermocouples, bubblers, and laser) } \\
\text { Flow totalizers on major chemical } \\
\text { additions } \\
\text { Flow meter on transfer line } \\
\end{array}$ & Operating procedures & & E0 & 2 \\
\hline PEP-30-2-15 & 2 & $\begin{array}{l}\text { Incomplete/“"out of } \\
\text { spec" batch of } \\
\text { material. Lost } \\
\text { time for test. }\end{array}$ & $\begin{array}{l}\text { Delivery of "out of spec" } \\
\text { caustic results in wrong } \\
\text { batch specs. }\end{array}$ & 1 & 0 & 3 & $\begin{array}{l}\text { Require certification from vendor with } \\
\text { each batch }\end{array}$ & Operating procedures & & E0 & 2 \\
\hline PEP-30-2-16 & 2 & Operational upset. & $\begin{array}{l}\text { Low pressure in feed } \\
\text { preparation tank due to } \\
\text { plugging of ventilation } \\
\text { (closure of damper, } \\
\text { plugged) during transfer } \\
\text { out. }\end{array}$ & & 0 & $\mathrm{n} / \mathrm{a}$ & $\begin{array}{l}\text { Design of feed preparation tank to } \\
7.5 \text { psid external }\end{array}$ & $\mathrm{n} / \mathrm{a}$ & $\begin{array}{l}\text { System is designed } \\
\text { such that the pumps } \\
\text { cannot draw enough } \\
\text { to damage tank. }\end{array}$ & E0 & 0 \\
\hline PEP-30-2-17 & 2 & $\begin{array}{l}\text { Operational upset - } \\
\text { Damage to tank. }\end{array}$ & $\begin{array}{l}\text { Low pressure on feed } \\
\text { preparation tank due to } \\
\text { cooling with ventilation } \\
\text { blocked (closure of } \\
\text { damper, plugged). }\end{array}$ & & 0 & $\mathrm{n} / \mathrm{a}$ & $\begin{array}{l}\text { Design of feed preparation tank with } \\
\text { two vent paths } \\
\text { Stop or bore through on the damper to } \\
\text { prevent full closure }\end{array}$ & $\mathrm{n} / \mathrm{a}$ & & E0 & 0 \\
\hline
\end{tabular}


Table E.1. Hazard Analysis Worksheets

\begin{tabular}{|c|c|c|c|c|c|c|c|c|c|c|c|}
\hline $\begin{array}{l}\text { Scenario } \\
\text { Number }\end{array}$ & Node & $\begin{array}{c}\text { Hazardous } \\
\text { Condition }\end{array}$ & Cause & $\mathbf{L}$ & C & $\begin{array}{l}\text { Risk } \\
\text { Score }\end{array}$ & Potential Safeguard & $\begin{array}{c}\text { Credited } \\
\text { Safeguards }\end{array}$ & $\begin{array}{c}\text { Comments/ } \\
\text { Assumptions }\end{array}$ & $\mathbf{E}$ & $\$$ \\
\hline PEP-30-2-18 & 2 & \begin{tabular}{|l|} 
Failure to tank and \\
spill of tank \\
contents.
\end{tabular} & $\begin{array}{l}\text { High pressure in feed } \\
\text { preparation tank due to } \\
\text { ventilation blocked } \\
\text { (closure of damper, } \\
\text { plugged) and overfilling } \\
\text { of tank. }\end{array}$ & -2 & 5 & 3 & $\begin{array}{l}\text { Pressure relief valve } \\
\text { Local pressure indicator for tank } \\
\text { Operating procedures } \\
\text { Pressure transmitter with AH \& AHH } \\
\text { on tanks } \\
\text { Stop or bore through on the damper to } \\
\text { prevent full closure }\end{array}$ & $\begin{array}{l}\text { PSV on Feed } \\
\text { Preparation tank } \\
\text { Stop or bore through } \\
\text { on the damper to } \\
\text { prevent full closure }\end{array}$ & $\begin{array}{l}\text { Spill to floor of } \\
\text { facility. }\end{array}$ & E0 & $\mathrm{SD}$ \\
\hline PEP-30-2-19 & 2 & $\begin{array}{l}\text { Failure to tank } \\
\text { spill of tank } \\
\text { contents. }\end{array}$ & $\begin{array}{l}\text { High pressure in feed } \\
\text { preparation tank due to } \\
\text { ventilation blocked } \\
\text { (closure of damper, } \\
\text { plugged) and tank } \\
\text { overheating due to } \\
\text { temperature control } \\
\text { system failure. }\end{array}$ & -1 & 5 & 4 & $\begin{array}{l}\text { Pressure relief valve on feed } \\
\text { preparation tank } \\
\text { Local pressure indicator for tank } \\
\text { Temperature controls } \\
\text { Ejector steam flow indicator } \\
\text { Operating procedures } \\
\text { Pressure transmitter with AH \& AHH } \\
\text { on tanks } \\
\text { Stop or bore through on the damper to } \\
\text { prevent full closure }\end{array}$ & $\begin{array}{l}\text { PSV on Feed } \\
\text { Preparation tank } \\
\text { Stop or bore through } \\
\text { on the damper to } \\
\text { prevent full closure. }\end{array}$ & $\begin{array}{l}\text { Spill to floor of } \\
\text { facility. Steam } \\
\text { release is potential } \\
\text { when heating. }\end{array}$ & E1 & $\mathrm{SD}$ \\
\hline PEP-30-2-20 & 2 & Operational upset. & $\begin{array}{l}\text { Lost heat trace on caustic } \\
\text { addition line. Plug line } \\
\text { with material-lose } \\
\text { caustic to receipt tanks. }\end{array}$ & & 0 & $\mathrm{n} / \mathrm{a}$ & & $\mathrm{n} / \mathrm{a}$ & & E0 & 0 \\
\hline PEP-30-2-21 & 2 & Operational upset. & $\begin{array}{l}\text { High temperature in feed } \\
\text { preparation tank due to } \\
\text { overheating. Boiling } \\
\text { contents of tank creates } \\
\text { excess aerosols. Excess } \\
\text { aerosols enter ventilation } \\
\text { system. }\end{array}$ & & 0 & $\mathrm{n} / \mathrm{a}$ & Temperature indicators & $\mathrm{n} / \mathrm{a}$ & $\begin{array}{l}\text { The aerosols } \\
\text { generated during } \\
\text { boiling will not } \\
\text { exceed environmental } \\
\text { permit limits. }\end{array}$ & E0 & 0 \\
\hline PEP-30-2-23 & 2 & Operational upset. & $\begin{array}{l}\text { PJMs on feed preparation } \\
\text { tank does not operate or } \\
\text { have poor performance. }\end{array}$ & & 0 & $\mathrm{n} / \mathrm{a}$ & & $\mathrm{n} / \mathrm{a}$ & & E0 & 0 \\
\hline PEP-30-2-24 & 2 & \begin{tabular}{|l|} 
Carryover of \\
material into the \\
ventilation system \\
and on to floor. \\
\end{tabular} & $\begin{array}{l}\text { PJMs in feed preparation } \\
\text { tank causes foaming in the } \\
\text { receipt tank. }\end{array}$ & 1 & 0 & 3 & \begin{tabular}{|l|} 
Sightglass \\
Operating procedures \\
Anti-foaming agent \\
Knockout pot \\
\end{tabular} & None identified & & E1 & 2 \\
\hline PEP-30-2-25 & 2 & Operational upset. & \begin{tabular}{|l} 
Prolonged settling in feed \\
preparation tank causes \\
accumulation of solids in \\
tank and results in \\
difficulty in moving/ \\
mixing material.
\end{tabular} & & 0 & $\mathrm{n} / \mathrm{a}$ & Operating procedures & $\mathrm{n} / \mathrm{a}$ & & E0 & 0 \\
\hline
\end{tabular}


Table E.1. Hazard Analysis Worksheets

\begin{tabular}{|c|c|c|c|c|c|c|c|c|c|c|c|}
\hline $\begin{array}{l}\text { Scenario } \\
\text { Number } \\
\end{array}$ & Node & $\begin{array}{c}\text { Hazardous } \\
\text { Condition } \\
\end{array}$ & Cause & L & C & $\begin{array}{c}\text { Risk } \\
\text { Score } \\
\end{array}$ & Potential Safeguard & $\begin{array}{c}\text { Credited } \\
\text { Safeguards } \\
\end{array}$ & $\begin{array}{c}\text { Comments/ } \\
\text { Assumptions }\end{array}$ & $\mathbf{E}$ & $\$$ \\
\hline PEP-30-2-26 & 2 & $\begin{array}{l}\text { Incomplete/“out of } \\
\text { spec" batch of } \\
\text { material. Lost } \\
\text { time for test. }\end{array}$ & $\begin{array}{l}\text { Failure in heat exchanger } \\
\text { causes leak of chilled } \\
\text { water or steam into } \\
\text { process line and causes } \\
\text { dilution of simulant } \\
\text { mixture. }\end{array}$ & -2 & 0 & 0 & & $\mathrm{n} / \mathrm{a}$ & & E0 & 2 \\
\hline PEP-30-2-27 & 2 & $\begin{array}{l}\text { Contaminate/ } \\
\text { damage the chilled } \\
\text { water or steam } \\
\text { supply system. }\end{array}$ & $\begin{array}{l}\text { Failure in heat exchanger } \\
\text { causes leak of process } \\
\text { material into chilled water } \\
\text { or steam supply. } \\
\text { Simulant mixture plugs } \\
\text { system. }\end{array}$ & -2 & 0 & 1 & $\begin{array}{l}\text { Condensate collection system and } \\
\text { blowdown on steam system } \\
\text { Periodic chemistry test on chilled } \\
\text { water system } \\
\text { TDS monitoring on steam supply } \\
\text { Water chemistry maintenance }\end{array}$ & Operating procedures & $\begin{array}{l}\text { Contamination in the } \\
\text { system would take } \\
\text { significant time } \\
\text { before damage would } \\
\text { occur. }\end{array}$ & E0 & 3 \\
\hline PEP-30-2-28 & 2 & Operational upset. & $\begin{array}{l}\text { High temperature in the } \\
\text { heating heat exchanger. } \\
\text { Lose flow of simulant } \\
\text { with steam in heat } \\
\text { exchanger. Dry out } \\
\text { system depositing scale } \\
\text { from heat exchanger to } \\
\text { feed preparation tank. } \\
\text { Plug system. }\end{array}$ & & 0 & $\mathrm{n} / \mathrm{a}$ & $\begin{array}{l}\text { Flushing capability for lines } \\
\text { Temperature monitoring } \\
\text { Operating procedures }\end{array}$ & $\mathrm{n} / \mathrm{a}$ & & E0 & 0 \\
\hline PEP-30-2-29 & 2 & Operational upset. & $\begin{array}{l}\text { Low temperatures in the } \\
\text { cooling heat exchanger } \\
\text { due to stopping flow in } \\
\text { the process loop. } \\
\text { Precipitate oxalate and } \\
\text { other solids plugging } \\
\text { exchanger. }\end{array}$ & & 0 & $\mathrm{n} / \mathrm{a}$ & $\begin{array}{l}\text { Flushing capability for lines } \\
\text { Temperature monitoring } \\
\text { Operating procedures }\end{array}$ & $\mathrm{n} / \mathrm{a}$ & & E0 & 0 \\
\hline PEP-30-2-30 & 2 & $\begin{array}{l}\text { Damage the heat } \\
\text { exchanger loop } \\
\text { releasing heated } \\
\text { process material. }\end{array}$ & $\begin{array}{l}\text { Closing isolation valves to } \\
\text { heat exchanger with } \\
\text { continued heating causes } \\
\text { over pressurization. }\end{array}$ & 1 & 5 & 6 & $\begin{array}{l}\text { Operating procedures } \\
\text { Pressure relief to the heat exchanger } \\
\text { Pressure Safety Valve }\end{array}$ & $\begin{array}{l}\text { Operating procedures } \\
\text { PSV on heat } \\
\text { exchanger }\end{array}$ & $\begin{array}{l}\text { Relief valve added at } \\
\text { the } 90 \% \text { design. }\end{array}$ & E0 & SD \\
\hline PEP-30-2-31 & 2 & $\begin{array}{l}\text { Large release of } \\
\text { steam to the } \\
\text { facility. }\end{array}$ & $\begin{array}{l}\text { Catastrophic failure of the } \\
\text { steam shell on the heat } \\
\text { exchanger results in large } \\
\text { release of steam. }\end{array}$ & -3 & 5 & 2 & Design of steam shell to code & $\begin{array}{l}\text { Design of steam shell } \\
\text { to code }\end{array}$ & & E0 & 3 \\
\hline PEP-30-2-32 & 2 & $\begin{array}{l}\text { Collapse of steam } \\
\text { shell. }\end{array}$ & $\begin{array}{l}\text { Steam present in the shell; } \\
\text { blocked off. Vacuum } \\
\text { pulled collapsing shell } \\
\text { upon cooling. }\end{array}$ & -1 & 0 & 2 & Design of steam shell to code & Operating procedures & & E0 & 3 \\
\hline
\end{tabular}


Table E.1. Hazard Analysis Worksheets

\begin{tabular}{|c|c|c|c|c|c|c|c|c|c|c|c|}
\hline $\begin{array}{l}\text { Scenario } \\
\text { Number }\end{array}$ & Node & $\begin{array}{l}\text { Hazardous } \\
\text { Condition }\end{array}$ & Cause & $\mathbf{L}$ & C & $\begin{array}{l}\text { Risk } \\
\text { Score }\end{array}$ & Potential Safeguard & $\begin{array}{l}\text { Credited } \\
\text { Safeguards }\end{array}$ & $\begin{array}{l}\text { Comments/ } \\
\text { Assumptions }\end{array}$ & $\mathbf{E}$ & $\$$ \\
\hline PEP-30-2-33 & 2 & $\begin{array}{l}\text { Small release of } \\
\text { steam to the } \\
\text { facility. }\end{array}$ & $\begin{array}{l}\text { Structural failure of the } \\
\text { steam shell of exchanger. } \\
\text { Small leak of steam. }\end{array}$ & -1 & 2 & 1 & & None identified & & E0 & 1 \\
\hline PEP-90-2-01 & 2 & $\begin{array}{l}\text { Spill of simulant, } \\
\text { sodium hydroxide, } \\
\text { material, or } \\
\text { inhibited water } \\
\text { from other tanks, } \\
\text { to the facility. } \\
\end{array}$ & $\begin{array}{l}\text { Flush valves are open } \\
\text { during transfer, spilling } \\
\text { material to the facility. }\end{array}$ & 0 & 4 & 4 & $\begin{array}{l}\text { Facility secondary containment } \\
\text { Operating procedures }\end{array}$ & \begin{tabular}{|l|} 
PPE \\
Facility secondary \\
containment \\
Operating procedures \\
Feed Preparation \\
tank level detection \\
\end{tabular} & Added at $90 \%$ & E0 & 1 \\
\hline PEP-90-2-02 & 2 & Operational upset. & $\begin{array}{l}\text { Control valve is mis- } \\
\text { aligned during transfer, } \\
\text { resulting in material } \\
\text { remaining in the feed } \\
\text { preparation tank. }\end{array}$ & & 0 & $\mathrm{n} / \mathrm{a}$ & & $\mathrm{n} / \mathrm{a}$ & Added at 90\% & E0 & \\
\hline PEP-90-2-03 & 2 & \begin{tabular}{|l} 
Spill of simulant, \\
sodium hydroxide, \\
material, or \\
inhibited water \\
from other tanks, \\
to the facility.
\end{tabular} & $\begin{array}{l}\text { Control valve is mis- } \\
\text { aligned during } \\
\text { recirculation, resulting in } \\
\text { material traveling to } \\
\text { ultrafiltration feed vessel } \\
\text { (T02A). Overflow tank to } \\
\text { facility. }\end{array}$ & -1 & 4 & 3 & $\begin{array}{l}\text { Facility secondary containment } \\
\text { Operating procedures } \\
\text { PPE } \\
\text { Personnel training } \\
\text { Ultrafiltration feed tank level } \\
\text { detection (thermocouples, bubblers, } \\
\text { and laser) }\end{array}$ & \begin{tabular}{|l|} 
PPE \\
Facility secondary \\
containment \\
Operating procedures
\end{tabular} & Added at $90 \%$ & E0 & SD \\
\hline PEP-90-2-04 & $2 \mathrm{a}$ & $\begin{array}{l}\text { Personnel injury } \\
\text { due to debris } \\
\text { projectiles. }\end{array}$ & \begin{tabular}{|l|} 
Feeding PJMs with \\
100 psi air, open vacuum \\
valve, and overpressurize \\
the vacuum system. \\
Failure of vacuum system.
\end{tabular} & -1 & 5 & 4 & $\begin{array}{l}\text { PLC } \\
\text { PJM Level Detector } \\
\text { PJM Pressure Detector } \\
\text { PJM Rack Air Supply Valve }\end{array}$ & $\begin{array}{l}\text { PLC } \\
\text { PJM Level Detector } \\
\text { PJM Pressure } \\
\text { Detector } \\
\text { PJM Rack Air } \\
\text { Supply Valve } \\
\text { Feed Preparation } \\
\text { tank level detection }\end{array}$ & Added at 90\% & E0 & SD \\
\hline PEP-30-2-34 & $2 b$ & $\begin{array}{l}\text { Incomplete/“out of } \\
\text { spec" batch of } \\
\text { material. Lost } \\
\text { time for test. } \\
\end{array}$ & $\begin{array}{l}\text { Add too much water to } \\
\text { the feed preparation tank } \\
\text { (inhibited or condensate } \\
\text { from the sparger). }\end{array}$ & 1 & 0 & 3 & $\begin{array}{l}\text { Flow totalizer on inhibited water } \\
\text { Feed preparation tank level detection } \\
\text { (thermocouples, bubblers, and laser) } \\
\text { Steam flowmeter }\end{array}$ & $\begin{array}{l}\text { Operating procedures } \\
\text { Feed Preparation } \\
\text { tank level detection }\end{array}$ & $\begin{array}{l}\text { Changed ejector to } \\
\text { sparger at } 100 \%\end{array}$ & E0 & 2 \\
\hline PEP-30-2-35 & $2 b$ & Operational upset. & $\begin{array}{l}\text { No flow to the sparger } \\
\text { from plugged orifice or } \\
\text { loss of steam supply. Not } \\
\text { able to heat the feed } \\
\text { preparation tank with the } \\
\text { steam. }\end{array}$ & & 0 & $\mathrm{n} / \mathrm{a}$ & & $\mathrm{n} / \mathrm{a}$ & $\begin{array}{l}\text { Changed ejector to } \\
\text { sparger at } 100 \%\end{array}$ & E0 & 0 \\
\hline
\end{tabular}


Table E.1. Hazard Analysis Worksheets

\begin{tabular}{|c|c|c|c|c|c|c|c|c|c|c|c|}
\hline $\begin{array}{l}\text { Scenario } \\
\text { Number } \\
\end{array}$ & Node & $\begin{array}{c}\text { Hazardous } \\
\text { Condition } \\
\end{array}$ & Cause & $\mathbf{L}$ & C & $\begin{array}{c}\text { Risk } \\
\text { Score } \\
\end{array}$ & Potential Safeguard & $\begin{array}{l}\text { Credited } \\
\text { Safeguards }\end{array}$ & $\begin{array}{c}\text { Comments/ } \\
\text { Assumptions } \\
\end{array}$ & $\mathbf{E}$ & $\$$ \\
\hline PEP-30-2-36 & $2 b$ & $\begin{array}{l}\text { Large steam leak } \\
\text { outside of feed } \\
\text { preparation tank. }\end{array}$ & $\begin{array}{l}\text { Failure of the steam } \\
\text { system upstream of the } \\
\text { tank, resulting in large } \\
\text { release of steam. }\end{array}$ & -3 & 5 & 2 & $\begin{array}{l}\text { Design of steam system } \\
\text { Operating procedures }\end{array}$ & \begin{tabular}{|l|} 
Design of steam \\
system to code \\
Operating procedures
\end{tabular} & $\begin{array}{l}\text { Changed ejector to } \\
\text { sparger at } 100 \%\end{array}$ & E0 & $\mathrm{SD}$ \\
\hline PEP-30-2-37 & $2 \mathrm{~b}$ & $\begin{array}{l}\text { Small steam leak } \\
\text { outside of feed } \\
\text { preparation tank. }\end{array}$ & $\begin{array}{l}\text { Failure of the steam } \\
\text { system upstream of the } \\
\text { tank, resulting in small } \\
\text { release of steam. }\end{array}$ & 0 & 2 & 2 & $\begin{array}{l}\text { Design of steam system } \\
\text { Operating procedures }\end{array}$ & \begin{tabular}{|l|} 
Design of steam \\
system to code \\
Operating procedures
\end{tabular} & $\begin{array}{l}\text { Changed ejector to } \\
\text { sparger at } 100 \%\end{array}$ & E0 & $\mathrm{SD}$ \\
\hline PEP-FN-2-01 & $2 \mathrm{~b}$ & \begin{tabular}{|l|} 
Carryover of \\
material to the \\
ventilation system.
\end{tabular} & $\begin{array}{l}\text { Failure of steam sparger } \\
\text { in the tank. As tank } \\
\text { material is disturbed, it } \\
\text { entrains } \\
\text { simulant/chemicals into } \\
\text { steam volume. Carry } \\
\text { material up into the } \\
\text { ventilation system. }\end{array}$ & -3 & 0 & -1 & $\begin{array}{l}\text { Design of the steam system } \\
\text { Knock out pots }\end{array}$ & None identified & & E1 & 2 \\
\hline PEP-30-2-40 & $2 \mathrm{a}$ & Operational upset. & $\begin{array}{l}\text { No/low flow through } \\
\text { pulse jet mixers. }\end{array}$ & & 0 & $\mathrm{n} / \mathrm{a}$ & & $\mathrm{n} / \mathrm{a}$ & & E0 & 0 \\
\hline PEP-30-2-41 & $2 a$ & \begin{tabular}{|l|} 
Carryover of \\
material to the \\
ventilation system.
\end{tabular} & $\begin{array}{l}\text { High flow through the } \\
\text { PJMs results in overblow. }\end{array}$ & 1 & 0 & 3 & $\begin{array}{l}\text { PLC } \\
\text { PJM Level Detector } \\
\text { PJM Pressure Detector } \\
\text { PJM Rack Air Supply Valve }\end{array}$ & $\begin{array}{l}\text { PLC } \\
\text { PJM Level Detector } \\
\text { PJM Pressure } \\
\text { Detector } \\
\text { PJM Rack Air } \\
\text { Supply Valve } \\
\text { Feed Preparation } \\
\text { tank level detection } \\
\end{array}$ & $\begin{array}{l}\text { Revised at } 90 \% \text { to } \\
\text { reflect PJM overblow } \\
\text { controls }\end{array}$ & E1 & 2 \\
\hline PEP-30-2-42 & $2 \mathrm{a}$ & Operational upset. & $\begin{array}{l}\text { Pull vacuum too long on } \\
\text { PJM. Pull simulant into } \\
\text { vacuum system, damaging } \\
\text { system. }\end{array}$ & & 0 & $\mathrm{n} / \mathrm{a}$ & Design of barometric leg & $\mathrm{n} / \mathrm{a}$ & & E0 & 0 \\
\hline PEP-30-2-43 & $2 a$ & \begin{tabular}{|l|} 
Carryover of \\
material to the \\
ventilation system.
\end{tabular} & $\begin{array}{l}\text { High pressure on the } \\
\text { vacuum side or high } \\
\text { pressure on the air side } \\
\text { results in overblow. }\end{array}$ & 1 & 0 & 3 & $\begin{array}{l}\text { PLC } \\
\text { PJM Level Detector } \\
\text { PJM Pressure Detector } \\
\text { PJM Rack Air Supply Valve }\end{array}$ & $\begin{array}{l}\text { PLC } \\
\text { PJM Level Detector } \\
\text { PJM Pressure } \\
\text { Detector } \\
\text { PJM Rack Air } \\
\text { Supply Valve } \\
\text { Feed Preparation } \\
\text { tank level detection }\end{array}$ & $\begin{array}{l}\text { Revised at } 90 \% \text { to } \\
\text { reflect PJM overblow } \\
\text { controls }\end{array}$ & E1 & 2 \\
\hline PEP-30-2-44 & $2 a$ & Operational upset. & $\begin{array}{l}\text { Vacuum results in boiling } \\
\text { at the PJMs due to high } \\
\text { temperature of material, } \\
\text { resulting in vapors and }\end{array}$ & & 0 & $\mathrm{n} / \mathrm{a}$ & $\begin{array}{l}\text { Design feature in the vacuum system } \\
\text { to handle condensables. }\end{array}$ & $\mathrm{n} / \mathrm{a}$ & & E0 & 0 \\
\hline
\end{tabular}


Table E.1. Hazard Analysis Worksheets

\begin{tabular}{|c|c|c|c|c|c|c|c|c|c|c|c|}
\hline $\begin{array}{l}\text { Scenario } \\
\text { Number } \\
\end{array}$ & Node & $\begin{array}{c}\text { Hazardous } \\
\text { Condition }\end{array}$ & Cause & $\mathbf{L}$ & C & $\begin{array}{c}\text { Risk } \\
\text { Score } \\
\end{array}$ & Potential Safeguard & $\begin{array}{c}\text { Credited } \\
\text { Safeguards } \\
\end{array}$ & $\begin{array}{c}\text { Comments/ } \\
\text { Assumptions }\end{array}$ & E & $\$$ \\
\hline & & & $\begin{array}{l}\text { condensate accumulating } \\
\text { in vacuum system. }\end{array}$ & & & & & & & & \\
\hline PEP-30-2-45 & $2 a$ & Operational upset. & $\begin{array}{l}\text { Lose structural integrity } \\
\text { of the air supply to the } \\
\text { PJMs. Blow air out into } \\
\text { tank at } 100 \mathrm{psi}(24 \mathrm{cfm}) .\end{array}$ & & 0 & $\mathrm{n} / \mathrm{a}$ & & $\mathrm{n} / \mathrm{a}$ & & E0 & 0 \\
\hline PEP-30-3-01 & 3 & Operational upset. & $\begin{array}{l}\text { No flow into the } \\
\text { ultrafiltration feed tank } \\
\text { due to closed isolation } \\
\text { valve. }\end{array}$ & & 0 & $\mathrm{n} / \mathrm{a}$ & & $n / a$ & & E0 & 0 \\
\hline PEP-30-3-02 & 3 & $\begin{array}{l}\text { Spill of simulant, } \\
\text { sodium hydroxide, } \\
\text { nitric acid/oxalic } \\
\text { acid, material, or } \\
\text { inhibited water } \\
\text { from other tanks. }\end{array}$ & $\begin{array}{l}\text { No flow into the tank due } \\
\text { to leak in chemical } \\
\text { addition or transfer piping } \\
\text { (flanges, etc.). }\end{array}$ & 0 & 4 & 4 & $\begin{array}{l}\text { Leak test at installation } \\
\text { Facility secondary containment } \\
\text { Operating procedures } \\
\text { PPE } \\
\text { Personnel training } \\
\text { Ultrafiltration feed tank level } \\
\text { detection (thermocouples, bubblers, } \\
\text { and laser) } \\
\text { Flow totalizers on major chemical } \\
\text { additions } \\
\text { Flow meter on transfer line } \\
\end{array}$ & \begin{tabular}{|l|} 
PPE \\
Operating procedures \\
Facility secondary \\
containment \\
Ultrafiltration feed \\
tank level detection
\end{tabular} & $\begin{array}{l}\text { Potential for } \\
\text { caustic/acidic } \\
\text { burn/eye damage. } \\
\text { Leak is inside facility. }\end{array}$ & E0 & 1 \\
\hline PEP-30-3-03 & 3 & Operational upset. & $\begin{array}{l}\text { Add acid to tank when } \\
\text { simulant is present, } \\
\text { causing tank to boil due to } \\
\text { reaction. Release aerosols } \\
\text { to ventilation system. }\end{array}$ & & 0 & $\mathrm{n} / \mathrm{a}$ & & $\mathrm{n} / \mathrm{a}$ & $\begin{array}{l}\text { This is } 2 \text { Molar nitric } \\
\text { acid. } \\
\text { The aerosols } \\
\text { generated during boil } \\
\text { will not exceed } \\
\text { environmental permit } \\
\text { limits. }\end{array}$ & E0 & 0 \\
\hline PEP-30-3-04 & 3 & Operational upset. & \begin{tabular}{|l|} 
No flow into the \\
ultrafiltration feed tank \\
due to pump failure or \\
plugging of chemical \\
addition or transfer piping \\
(flanges, etc.).
\end{tabular} & & 0 & $\mathrm{n} / \mathrm{a}$ & & $\mathrm{n} / \mathrm{a}$ & & E0 & 0 \\
\hline PEP-30-3-05 & 3 & Operational upset. & $\begin{array}{l}\text { No flow out of } \\
\text { ultrafiltration feed tank } \\
\text { due to pump not } \\
\text { operating. }\end{array}$ & & 0 & $\mathrm{n} / \mathrm{a}$ & & $\mathrm{n} / \mathrm{a}$ & & E0 & 0 \\
\hline PEP-30-3-06 & 3 & Operational upset. & $\begin{array}{l}\text { No flow out of } \\
\text { ultrafiltration feed tank } \\
\text { due to line plugging. }\end{array}$ & & 0 & $\mathrm{n} / \mathrm{a}$ & & $\mathrm{n} / \mathrm{a}$ & & E0 & 0 \\
\hline
\end{tabular}


Table E.1. Hazard Analysis Worksheets

\begin{tabular}{|c|c|c|c|c|c|c|c|c|c|c|c|}
\hline $\begin{array}{l}\text { Scenario } \\
\text { Number }\end{array}$ & Node & $\begin{array}{c}\text { Hazardous } \\
\text { Condition } \\
\end{array}$ & Cause & L & C & $\begin{array}{c}\text { Risk } \\
\text { Score } \\
\end{array}$ & Potential Safeguard & $\begin{array}{c}\text { Credited } \\
\text { Safeguards } \\
\end{array}$ & $\begin{array}{c}\text { Comments/ } \\
\text { Assumptions }\end{array}$ & $\mathbf{E}$ & $\$$ \\
\hline PEP-30-3-07 & 3 & $\begin{array}{l}\text { Spill of } \\
\text { ultrafiltration tank } \\
\text { contents (simulant, } \\
\text { sodium hydroxide, } \\
\text { nitric acid/oxalic } \\
\text { acid, material, or } \\
\text { inhibited water } \\
\text { from other tanks). }\end{array}$ & $\begin{array}{l}\text { Structural integrity of } \\
\text { ultrafiltration feed tank } \\
\text { degraded, resulting in } \\
\text { leak. }\end{array}$ & -3 & 4 & 1 & Design of tanks to code & $\begin{array}{l}\text { Design of } \\
\text { ultrafiltration feed } \\
\text { tank to code }\end{array}$ & $\begin{array}{l}\text { Spill to floor of } \\
\text { facility. } \\
\text { Potential for caustic } \\
\text { burn/eye damage } \\
\text { pH 14. } \\
\text { Likelihood assigned } \\
\text { based on tank to code. } \\
304 \text { SST is listed as } \\
\text { having excellent } \\
\text { chemical } \\
\text { compatibility to nitric } \\
\text { acid and good } \\
\text { compatibility with } \\
\text { NaOH and oxalic } \\
\text { acid. }\end{array}$ & E0 & 3 \\
\hline PEP-30-3-08 & 3 & $\begin{array}{l}\text { Spill of } \\
\text { ultrafiltration tank } \\
\text { contents (simulant, } \\
\text { sodium hydroxide, } \\
\text { nitric acid/oxalic } \\
\text { acid, material, or } \\
\text { inhibited water } \\
\text { from other tanks). }\end{array}$ & $\begin{array}{l}\text { No flow out of the } \\
\text { ultrafiltration feed tank } \\
\text { due to leak in transfer } \\
\text { piping/pumps. }\end{array}$ & 0 & 4 & 4 & \begin{tabular}{|l|} 
Leak test at installation \\
Facility secondary containment \\
Operating procedures \\
PPE \\
Personnel training \\
Ultrafiltration feed tank level \\
detection (thermocouple, bubblers and \\
laser) \\
Flow meter on transfer line \\
\end{tabular} & $\begin{array}{l}\text { PPE } \\
\text { Operating procedures } \\
\text { Facility secondary } \\
\text { containment } \\
\text { Ultrafiltration feed } \\
\text { tank level detection }\end{array}$ & $\begin{array}{l}\text { Spill to floor of } \\
\text { facility. } \\
\text { Potential for } \\
\text { caustic/acidic } \\
\text { burn/eye damage. } \\
\text { Likelihood assigned } \\
\text { based on tank to code. }\end{array}$ & E0 & SD \\
\hline PEP-30-3-09 & 3 & \begin{tabular}{|l} 
Carryover of \\
material to the \\
ventilation system.
\end{tabular} & $\begin{array}{l}\text { Lower-than-intended } \\
\text { ultrafiltration feed tank } \\
\text { volume. Tank level } \\
\text { below level of the PJMs } \\
\text { results in overblow. } \\
\text { Material in the vent line. }\end{array}$ & 1 & 0 & 3 & $\begin{array}{l}\text { PLC } \\
\text { PJM Level Detector } \\
\text { PJM Pressure Detector } \\
\text { PJM Rack Air Supply Valve }\end{array}$ & $\begin{array}{l}\text { PLC } \\
\text { PJM Level Detector } \\
\text { PJM Pressure } \\
\text { Detector } \\
\text { PJM Rack Air } \\
\text { Supply Valve } \\
\text { Ultrafiltration feed } \\
\text { tank level detection }\end{array}$ & $\begin{array}{l}\text { Revised at } 90 \% \text { to } \\
\text { reflect PJM overblow } \\
\text { controls. }\end{array}$ & E1 & 2 \\
\hline PEP-30-3-10 & 3 & \begin{tabular}{|l|} 
No issue \\
identified.
\end{tabular} & Filling tank too fast. & & 0 & $\mathrm{n} / \mathrm{a}$ & & $\mathrm{n} / \mathrm{a}$ & $\begin{array}{l}\text { Ventilation system is } \\
\text { designed to } \\
\text { accommodate for any } \\
\text { excess aerosols } \\
\text { generated during this } \\
\text { event. }\end{array}$ & E0 & 0 \\
\hline
\end{tabular}


Table E.1. Hazard Analysis Worksheets

\begin{tabular}{|c|c|c|c|c|c|c|c|c|c|c|c|}
\hline $\begin{array}{l}\text { Scenario } \\
\text { Number }\end{array}$ & Node & $\begin{array}{c}\text { Hazardous } \\
\text { Condition }\end{array}$ & Cause & $\mathbf{L}$ & $\mathrm{C}$ & $\begin{array}{l}\text { Risk } \\
\text { Score }\end{array}$ & Potential Safeguard & $\begin{array}{c}\text { Credited } \\
\text { Safeguards }\end{array}$ & $\begin{array}{c}\text { Comments/ } \\
\text { Assumptions }\end{array}$ & $\mathbf{E}$ & $\$$ \\
\hline PEP-30-3-11 & 3 & Operational upset. & \begin{tabular}{|l|} 
Too much material added, \\
resulting in boiling in \\
ultrafiltration feed tank. \\
Excess aerosols enter \\
ventilation system. \\
\end{tabular} & & 0 & $\mathrm{n} / \mathrm{a}$ & & $\mathrm{n} / \mathrm{a}$ & $\begin{array}{l}\text { Aerosols generated } \\
\text { during boiling will } \\
\text { not exceed } \\
\text { environment permit } \\
\text { limits. } \\
\end{array}$ & E0 & 0 \\
\hline PEP-30-3-12 & 3 & $\begin{array}{l}\text { Spill of } \\
\text { ultrafiltration tank } \\
\text { contents (simulant, } \\
\text { sodium hydroxide, } \\
\text { nitric acid/oxalic } \\
\text { acid, material, or } \\
\text { inhibited water } \\
\text { from other tanks). }\end{array}$ & $\begin{array}{l}\text { Too much material added } \\
\text { to the ultrafiltration tank } \\
\text { resulting in overflow of } \\
\text { tank to facility floor. }\end{array}$ & 0 & 4 & 4 & $\begin{array}{l}\text { Facility secondary containment } \\
\text { Operating procedures } \\
\text { PPE } \\
\text { Personnel training } \\
\text { Ultrafiltration feed tank level } \\
\text { detection (thermocouples, bubblers, } \\
\text { and laser) } \\
\text { Flow totalizers on major chemical } \\
\text { additions } \\
\text { Flow meter on transfer line }\end{array}$ & \begin{tabular}{|l|} 
PPE \\
Operating procedures \\
Facility secondary \\
containment \\
Ultrafiltration feed \\
tank level detection \\
and alarms
\end{tabular} & $\begin{array}{l}\text { Spill to floor of } \\
\text { facility. } \\
\text { Potential for } \\
\text { caustic/acidic } \\
\text { burn/eye damage. }\end{array}$ & E0 & $\mathrm{SD}$ \\
\hline PEP-30-3-13 & 3 & $\begin{array}{l}\text { Incomplete/“out of } \\
\text { spec" batch of } \\
\text { material. Lost } \\
\text { time for test. }\end{array}$ & $\begin{array}{l}\text { Too much material added } \\
\text { to the tank. High level in } \\
\text { tank. }\end{array}$ & 1 & 0 & 3 & $\begin{array}{l}\text { Operating procedures } \\
\text { Ultrafiltration feed tank level } \\
\text { detection (thermocouples, bubblers, } \\
\text { and laser) } \\
\text { Flow totalizers on major chemical } \\
\text { additions } \\
\text { Flow meter on transfer line }\end{array}$ & $\begin{array}{l}\text { Ultrafiltration feed } \\
\text { tank level detection } \\
\text { and alarms }\end{array}$ & & E0 & 2 \\
\hline PEP-30-3-14 & 3 & Operational upset. & $\begin{array}{l}\text { Too much caustic added } \\
\text { causes formation of } \\
\text { oxalates in tank. }\end{array}$ & & 0 & $\mathrm{n} / \mathrm{a}$ & & $\mathrm{n} / \mathrm{a}$ & & E0 & 0 \\
\hline PEP-90-3-01 & 3 & $\begin{array}{l}\text { Spill of } \\
\text { ultrafiltration tank } \\
\text { contents (simulant, } \\
\text { sodium hydroxide, } \\
\text { nitric acid/oxalic } \\
\text { acid, material, or } \\
\text { inhibited water } \\
\text { from other tanks). }\end{array}$ & $\begin{array}{l}\text { Ultafiltration pump flush } \\
\text { valves left open during } \\
\text { pump operation. Pump } \\
\text { ultrafiltration tank } \\
\text { contents into drain pan, } \\
\text { overflowing pan into } \\
\text { facility. }\end{array}$ & 0 & 4 & 4 & $\begin{array}{l}\text { Facility secondary containment } \\
\text { Operating procedures } \\
\text { PPE } \\
\text { Personnel training } \\
\text { Ultrafiltration feed tank level } \\
\text { detection (thermocouples, bubblers, } \\
\text { and laser) }\end{array}$ & \begin{tabular}{|l|} 
PPE \\
Facility secondary \\
containment \\
Operating procedures \\
Ultrafiltration feed \\
tank level detection
\end{tabular} & Added at $90 \%$ & E0 & $\mathrm{SD}$ \\
\hline PEP-30-3-15 & 3 & $\begin{array}{l}\text { Incomplete/“out of } \\
\text { spec” batch of } \\
\text { material. Lost } \\
\text { time for test. }\end{array}$ & $\begin{array}{l}\text { Too little caustic added, } \\
\text { resulting in contents too } \\
\text { low in caustic through } \\
\text { ultrafiltration system. }\end{array}$ & 1 & 0 & 3 & $\begin{array}{l}\text { Operating procedures } \\
\text { Ultrafiltration feed tank level } \\
\text { detection (thermocouples, bubblers, } \\
\text { and laser) } \\
\text { Flow totalizers on major chemical } \\
\text { additions }\end{array}$ & Operating procedures & & E0 & 2 \\
\hline
\end{tabular}


Table E.1. Hazard Analysis Worksheets

\begin{tabular}{|c|c|c|c|c|c|c|c|c|c|c|c|}
\hline $\begin{array}{l}\text { Scenario } \\
\text { Number }\end{array}$ & Node & $\begin{array}{c}\text { Hazardous } \\
\text { Condition }\end{array}$ & Cause & $\mathbf{L}$ & C & $\begin{array}{l}\text { Risk } \\
\text { Score }\end{array}$ & Potential Safeguard & $\begin{array}{l}\text { Credited } \\
\text { Safeguards }\end{array}$ & $\begin{array}{l}\text { Comments/ } \\
\text { Assumptions }\end{array}$ & $\mathbf{E}$ & $\$$ \\
\hline PEP-30-3-16 & 3 & $\begin{array}{l}\text { Incomplete/“out of } \\
\text { spec" batch of } \\
\text { material. Lost } \\
\text { time for test. }\end{array}$ & $\begin{array}{l}\text { Delivery of "out of spec" } \\
\text { caustic results in wrong } \\
\text { batch specs. }\end{array}$ & 1 & 0 & 3 & $\begin{array}{l}\text { Require certification from vendor with } \\
\text { each batch. }\end{array}$ & Operating procedures & & E0 & 2 \\
\hline PEP-30-3-17 & 3 & Operational upset. & $\begin{array}{l}\text { Low pressure in } \\
\text { ultrafiltration feed tank } \\
\text { due to plugging of } \\
\text { ventilation (closure of } \\
\text { damper, plugged) during } \\
\text { transfer out. }\end{array}$ & & 0 & $\mathrm{n} / \mathrm{a}$ & $\begin{array}{l}\text { Design of tanks to } 7.5 \text { psid external } \\
\text { Stop or bore through on the damper to } \\
\text { prevent full closure. }\end{array}$ & $\mathrm{n} / \mathrm{a}$ & $\begin{array}{l}\text { System is designed } \\
\text { such that the pumps } \\
\text { cannot draw enough } \\
\text { suction to damage } \\
\text { tank. }\end{array}$ & E0 & 0 \\
\hline PEP-30-3-19 & 3 & \begin{tabular}{|l|} 
Failure of \\
ultrafiltration tank \\
and spill of tank \\
contents (simulant, \\
sodium hydroxide, \\
nitric acid/oxalic \\
acid, material, or \\
inhibited water \\
from other tanks). \\
\end{tabular} & $\begin{array}{l}\text { High pressure in } \\
\text { ultrafiltration feed tank } \\
\text { due to ventilation blocked } \\
\text { (closure of damper, } \\
\text { plugged) and overfilling } \\
\text { of tank. }\end{array}$ & -2 & 5 & 3 & $\begin{array}{l}\text { Pressure relief valve } \\
\text { Local pressure indicator for tank } \\
\text { Operating procedures } \\
\text { Pressure transmitter } \\
\text { Stop or bore through on the damper to } \\
\text { prevent full closure. }\end{array}$ & $\begin{array}{l}\text { PSV on } \\
\text { Ultrafiltration Feed } \\
\text { tank } \\
\text { Stop or bore through } \\
\text { on the damper to } \\
\text { prevent full closure }\end{array}$ & $\begin{array}{l}\text { Spill to floor of } \\
\text { facility. }\end{array}$ & E0 & $\mathrm{SD}$ \\
\hline PEP-30-3-20 & 3 & \begin{tabular}{|l|} 
Failure of \\
ultrafiltration tank \\
and spill of tank \\
contents (simulant, \\
sodium hydroxide, \\
nitric acid/oxalic \\
acid, material, or \\
inhibited water \\
from other tanks). \\
\end{tabular} & $\begin{array}{l}\text { High pressure in } \\
\text { ultrafiltration feed tank } \\
\text { due to ventilation blocked } \\
\text { (closure of damper, } \\
\text { plugged) and overheat the } \\
\text { tank due to temperature } \\
\text { control system failure. }\end{array}$ & -1 & 5 & 4 & $\begin{array}{l}\text { Pressure relief valve } \\
\text { Local pressure indicator for tank } \\
\text { Temperature controls } \\
\text { Ejector steam flow indicator } \\
\text { Operating procedures } \\
\text { Pressure transmitter } \\
\text { Stop or bore through on the damper to } \\
\text { prevent full closure. }\end{array}$ & $\begin{array}{l}\text { PSV on } \\
\text { Ultrafiltration Feed } \\
\text { tank } \\
\text { Stop or bore through } \\
\text { on the damper to } \\
\text { prevent full closure. }\end{array}$ & $\begin{array}{l}\text { Spill to floor of } \\
\text { facility. Steam } \\
\text { release is potential } \\
\text { when heating. }\end{array}$ & E0 & $\mathrm{SD}$ \\
\hline PEP-30-3-21 & 3 & Operational upset. & $\begin{array}{l}\text { Lost heat trace on caustic } \\
\text { addition line. Plug line } \\
\text { with material-lose } \\
\text { caustic to receipt tanks. }\end{array}$ & & 0 & $\mathrm{n} / \mathrm{a}$ & & $\mathrm{n} / \mathrm{a}$ & & E0 & 0 \\
\hline PEP-30-3-22 & 3 & Operational upset. & $\begin{array}{l}\text { High temperature in } \\
\text { ultrafiltration feed tank } \\
\text { due to overheating. } \\
\text { Boiling contents of tank, } \\
\text { creates excess aerosols. } \\
\text { Excess aerosols enter } \\
\text { ventilation system. }\end{array}$ & & 0 & $\mathrm{n} / \mathrm{a}$ & Temperature indicators & $\mathrm{n} / \mathrm{a}$ & $\begin{array}{l}\text { The aerosols } \\
\text { generated during } \\
\text { boiling will not } \\
\text { exceed environmental } \\
\text { permit limits. }\end{array}$ & E0 & 0 \\
\hline PEP-30-3-24 & 3 & Operational upset. & $\begin{array}{l}\text { PJM does not operate or } \\
\text { has poor performance. }\end{array}$ & & 0 & $\mathrm{n} / \mathrm{a}$ & & $\mathrm{n} / \mathrm{a}$ & & E0 & 0 \\
\hline
\end{tabular}


Table E.1. Hazard Analysis Worksheets

\begin{tabular}{|c|c|c|c|c|c|c|c|c|c|c|c|}
\hline $\begin{array}{l}\text { Scenario } \\
\text { Number }\end{array}$ & Node & $\begin{array}{c}\text { Hazardous } \\
\text { Condition } \\
\end{array}$ & Cause & $\mathbf{L}$ & C & $\begin{array}{l}\text { Risk } \\
\text { Score } \\
\end{array}$ & Potential Safeguard & $\begin{array}{c}\text { Credited } \\
\text { Safeguards } \\
\end{array}$ & $\begin{array}{l}\text { Comments/ } \\
\text { Assumptions }\end{array}$ & E & $\$$ \\
\hline PEP-30-3-25 & 3 & \begin{tabular}{|l|} 
Carryover of \\
material into the \\
ventilation system \\
and on to floor.
\end{tabular} & $\begin{array}{l}\text { PJM causes foaming in } \\
\text { the receipt tank. }\end{array}$ & 1 & 0 & 3 & $\begin{array}{l}\text { Operating procedures } \\
\text { Anti-foaming agent } \\
\text { Knockout pot }\end{array}$ & None identified & & E1 & 2 \\
\hline PEP-30-3-26 & 3 & Operational upset. & $\begin{array}{l}\text { Prolonged settling in tank } \\
\text { causes accumulation of } \\
\text { solids in tank and results } \\
\text { in difficulty moving/ } \\
\text { mixing of material. } \\
\end{array}$ & & 0 & $\mathrm{n} / \mathrm{a}$ & Operating procedures & $\mathrm{n} / \mathrm{a}$ & & E0 & 0 \\
\hline PEP-30-3-27 & 3 & $\begin{array}{l}\text { Incomplete/"out of } \\
\text { spec" batch of } \\
\text { material. Lost } \\
\text { time for test. }\end{array}$ & $\begin{array}{l}\text { Failure in heat exchanger } \\
\text { causes leak of chilled } \\
\text { water or steam into } \\
\text { process line and causes } \\
\text { dilution of simulant } \\
\text { mixture. }\end{array}$ & -2 & 0 & 0 & & None identified & & E0 & 2 \\
\hline PEP-30-3-28 & $3 b$ & $\begin{array}{l}\text { Incomplete/"out of } \\
\text { spec" batch of } \\
\text { material. Lost } \\
\text { time for test. }\end{array}$ & $\begin{array}{l}\text { Add too much water to } \\
\text { ultrafiltration feed tank } \\
\text { (inhibited or condensate } \\
\text { from the sparger). }\end{array}$ & 1 & 0 & 3 & $\begin{array}{l}\text { Flow totalizer on inhibited water } \\
\text { Ultrafiltration feed tank level } \\
\text { detection (thermocouples, bubblers, } \\
\text { and laser) } \\
\text { Steam Flowmeter } \\
\end{array}$ & Operating procedures & $\begin{array}{l}\text { Changed ejector to } \\
\text { sparger at } 100 \%\end{array}$ & E0 & 2 \\
\hline PEP-30-3-29 & $3 b$ & Operational upset. & $\begin{array}{l}\text { No flow to the spargers } \\
\text { from plugged orifice or } \\
\text { loss of steam supply. Not } \\
\text { able to heat the tank with } \\
\text { steam. }\end{array}$ & & 0 & $\mathrm{n} / \mathrm{a}$ & & $\mathrm{n} / \mathrm{a}$ & $\begin{array}{l}\text { Changed ejector to } \\
\text { sparger at } 100 \%\end{array}$ & E0 & 0 \\
\hline PEP-30-3-30 & $3 b$ & $\begin{array}{l}\text { Large steam leak } \\
\text { outside of tank. }\end{array}$ & $\begin{array}{l}\text { Failure of the steam } \\
\text { system upstream of the } \\
\text { tank, resulting in large } \\
\text { release of steam. }\end{array}$ & -3 & 5 & 2 & $\begin{array}{l}\text { Design of steam system } \\
\text { Operating procedures }\end{array}$ & \begin{tabular}{|l|} 
Design of steam \\
system to code \\
Operating procedures
\end{tabular} & $\begin{array}{l}\text { Changed ejector to } \\
\text { sparger at } 100 \%\end{array}$ & E0 & SD \\
\hline PEP-30-3-31 & $3 b$ & $\begin{array}{l}\text { Small steam leak } \\
\text { outside of tank. }\end{array}$ & $\begin{array}{l}\text { Failure of the steam } \\
\text { system upstream of the } \\
\text { tank, resulting in small } \\
\text { release of steam. }\end{array}$ & 0 & 2 & 2 & $\begin{array}{l}\text { Design of steam system } \\
\text { Operating procedures }\end{array}$ & \begin{tabular}{|l|} 
Design of steam \\
system to code \\
Operating procedures
\end{tabular} & $\begin{array}{l}\text { Changed ejector to } \\
\text { sparger at } 100 \%\end{array}$ & E0 & SD \\
\hline PEP-FN-3-01 & $3 b$ & \begin{tabular}{|l|} 
Carryover of \\
material to the \\
ventilation system.
\end{tabular} & $\begin{array}{l}\text { Failure of steam sparger } \\
\text { in the tank. As tank } \\
\text { material is disturbed in } \\
\text { entrains simulant/ } \\
\text { chemicals into steam } \\
\text { volume. Carry material } \\
\text { up into the ventilation } \\
\text { system. }\end{array}$ & -3 & 0 & -3 & $\begin{array}{l}\text { Design of the steam system } \\
\text { Knock out pots }\end{array}$ & None identified & & E1 & 2 \\
\hline
\end{tabular}


Table E.1. Hazard Analysis Worksheets

\begin{tabular}{|c|c|c|c|c|c|c|c|c|c|c|c|}
\hline $\begin{array}{l}\text { Scenario } \\
\text { Number }\end{array}$ & Node & $\begin{array}{l}\text { Hazardous } \\
\text { Condition }\end{array}$ & Cause & $\mathbf{L}$ & C & $\begin{array}{l}\text { Risk } \\
\text { Score }\end{array}$ & Potential Safeguard & $\begin{array}{c}\text { Credited } \\
\text { Safeguards }\end{array}$ & $\begin{array}{l}\text { Comments/ } \\
\text { Assumptions }\end{array}$ & $\mathbf{E}$ & $\$$ \\
\hline PEP-30-3-34 & $3 a$ & Operational upset. & $\begin{array}{l}\text { No/low flow through } \\
\text { pulse jet mixers. }\end{array}$ & & 0 & $\mathrm{n} / \mathrm{a}$ & & $\mathrm{n} / \mathrm{a}$ & & E0 & 0 \\
\hline PEP-30-3-35 & $3 a$ & \begin{tabular}{|l|} 
Carryover of \\
material to the \\
ventilation system.
\end{tabular} & $\begin{array}{l}\text { High flow through the } \\
\text { PJMs results in overblow. }\end{array}$ & 1 & 0 & 3 & $\begin{array}{l}\text { PLC } \\
\text { PJM Level Detector } \\
\text { PJM Pressure Detector } \\
\text { PJM Rack Air Supply Valve }\end{array}$ & $\begin{array}{l}\text { PLC } \\
\text { PJM Level Detector } \\
\text { PJM Pressure } \\
\text { Detector } \\
\text { PJM Rack Air } \\
\text { Supply Valve } \\
\text { Ultrafiltration feed } \\
\text { tank level detection }\end{array}$ & $\begin{array}{l}\text { Revised at } 90 \% \text { to } \\
\text { reflect PJM overblow } \\
\text { controls }\end{array}$ & E1 & 2 \\
\hline PEP-30-3-36 & $3 a$ & Operational upset. & $\begin{array}{l}\text { Pull vacuum too long on } \\
\text { PJM. Pull simulant into } \\
\text { vacuum system damaging } \\
\text { system. }\end{array}$ & & 0 & $\mathrm{n} / \mathrm{a}$ & Design of barometric leg & $\mathrm{n} / \mathrm{a}$ & & E0 & 0 \\
\hline PEP-30-3-37 & $3 a$ & \begin{tabular}{|l|} 
Carryover of \\
material to the \\
ventilation system.
\end{tabular} & $\begin{array}{l}\text { High pressure on the } \\
\text { vacuum side or high } \\
\text { pressure on the air side } \\
\text { results in overblow. }\end{array}$ & 1 & 0 & 3 & $\begin{array}{l}\text { PLC } \\
\text { PJM Level Detector } \\
\text { PJM Pressure Detector } \\
\text { PJM Rack Air Supply Valve }\end{array}$ & $\begin{array}{l}\text { PLC } \\
\text { PJM Level Detector } \\
\text { PJM Pressure } \\
\text { Detector } \\
\text { PJM Rack Air } \\
\text { Supply Valve } \\
\text { Ultrafiltration feed } \\
\text { tank level detection }\end{array}$ & $\begin{array}{l}\text { Revised at } 90 \% \text { to } \\
\text { reflect PJM overblow } \\
\text { controls }\end{array}$ & E1 & 2 \\
\hline PEP-30-3-38 & $3 a$ & Operational upset. & $\begin{array}{l}\text { Vacuum results in boiling } \\
\text { due to high temperature of } \\
\text { material, resulting in } \\
\text { vapors and condensate } \\
\text { accumulating in vacuum } \\
\text { system. }\end{array}$ & & 0 & $\mathrm{n} / \mathrm{a}$ & $\begin{array}{l}\text { Design feature in the vacuum system } \\
\text { to handle condensables. }\end{array}$ & $\mathrm{n} / \mathrm{a}$ & & E0 & 0 \\
\hline PEP-30-3-39 & $3 a$ & Operational upset. & $\begin{array}{l}\text { Lose structural integrity } \\
\text { of the air supply to the } \\
\text { PJMs. Blow air out into } \\
\text { tank at } 100 \text { psi }(24 \mathrm{cfm}) .\end{array}$ & & 0 & $\mathrm{n} / \mathrm{a}$ & & $\mathrm{n} / \mathrm{a}$ & & E0 & 0 \\
\hline PEP-30-3-40 & 3 & $\begin{array}{l}\text { Internal vessel and } \\
\text { jacket is damaged, } \\
\text { releasing hot } \\
\text { water. }\end{array}$ & $\begin{array}{l}\text { Block flow (close valves) } \\
\text { to the chilled water jacket } \\
\text { resulting in over } \\
\text { pressurization of jacket. }\end{array}$ & 1 & 3 & 5 & $\begin{array}{l}\text { Operating procedures } \\
\text { Flow meter on chilled water system } \\
\text { Pressure relief valve }\end{array}$ & $\begin{array}{l}\text { Operating procedures } \\
\text { PSV on chilled water } \\
\text { jacket }\end{array}$ & & E0 & 4 \\
\hline PEP-30-3-41 & 3 & \begin{tabular}{|l|} 
Spill ultrafiltration \\
tank contents to \\
the floor \\
(simulant, sodium \\
hydroxide, nitric \\
acid/oxalic acid, \\
\end{tabular} & $\begin{array}{l}\text { Failure of the chilled } \\
\text { water system and chilled } \\
\text { water leaking into the } \\
\text { ultrafiltration tank, } \\
\text { resulting in overflowing } \\
\text { the tank. }\end{array}$ & -2 & 4 & 2 & $\begin{array}{l}\text { Facility secondary containment } \\
\text { Operating procedures } \\
\text { PPE } \\
\text { Personnel training } \\
\text { Ultrafiltration feed tank level } \\
\text { detection (thermocouples, bubblers, }\end{array}$ & \begin{tabular}{|l} 
PPE \\
Facility secondary \\
containment \\
Ultrafiltration feed \\
tank level detection \\
and alarms
\end{tabular} & $\begin{array}{l}\text { Spill to floor of } \\
\text { facility. } \\
\text { Potential for } \\
\text { caustic/acid burn/eye } \\
\text { damage. }\end{array}$ & E0 & SD \\
\hline
\end{tabular}


Table E.1. Hazard Analysis Worksheets

\begin{tabular}{|c|c|c|c|c|c|c|c|c|c|c|c|}
\hline $\begin{array}{l}\text { Scenario } \\
\text { Number }\end{array}$ & Node & $\begin{array}{l}\text { Hazardous } \\
\text { Condition }\end{array}$ & Cause & $\mathbf{L}$ & C & $\begin{array}{l}\text { Risk } \\
\text { Score }\end{array}$ & Potential Safeguard & $\begin{array}{c}\text { Credited } \\
\text { Safeguards }\end{array}$ & $\begin{array}{c}\text { Comments/ } \\
\text { Assumptions }\end{array}$ & $\mathbf{E}$ & $\$$ \\
\hline & & \begin{tabular}{|l|} 
material, or \\
inhibited water \\
from other tanks).
\end{tabular} & & & & & $\begin{array}{l}\text { and laser) } \\
\text { Flow meter on chilled water }\end{array}$ & & & & \\
\hline PEP-30-3-42 & 3 & Operational upset. & $\begin{array}{l}\text { No air flow in the sparer } \\
\text { results in plugging of } \\
\text { sparger system. }\end{array}$ & & 0 & $\mathrm{n} / \mathrm{a}$ & $\begin{array}{l}\text { Flow totalizer on sparger } \\
\text { Local flowmeter }\end{array}$ & $\mathrm{n} / \mathrm{a}$ & & E0 & 0 \\
\hline PEP-30-3-43 & 3 & \begin{tabular}{|l|} 
Carryover of \\
material into the \\
ventilation system \\
and on to floor. \\
\end{tabular} & $\begin{array}{l}\text { Flow or air through } \\
\text { sparger to high. Results } \\
\text { in foaming and aerosol } \\
\text { generation. }\end{array}$ & 1 & 0 & 3 & $\begin{array}{l}\text { Operating procedures } \\
\text { Anti-foaming agent } \\
\text { Knockout pot } \\
\text { Flow totalizer on sparger }\end{array}$ & None identified & & E1 & 2 \\
\hline PEP-30-3-44 & 3 & \begin{tabular}{|l|} 
Carryover of \\
material into the \\
ventilation system \\
and on to floor.
\end{tabular} & $\begin{array}{l}\text { Regulator failure results } \\
\text { in high pressure flow } \\
\text { through sparger. }\end{array}$ & 1 & 0 & 3 & $\begin{array}{l}\text { Operating procedures } \\
\text { Anti-foaming agent } \\
\text { Knockout pot } \\
\text { Flow totalizer on sparger } \\
\text { Pressure indicator on the header }\end{array}$ & Operating procedures & & E1 & 2 \\
\hline PEP-30-3-45 & 3 & Operational upset. & $\begin{array}{l}\text { Lose structural integrity } \\
\text { of the air supply to the } \\
\text { sparger. Blow air out into } \\
\text { tank at } 15 \text { psi }(4 \mathrm{cfm}) .\end{array}$ & & 0 & $\mathrm{n} / \mathrm{a}$ & & $\mathrm{n} / \mathrm{a}$ & & E0 & 0 \\
\hline PEP-30-4-01 & $4 \mathrm{~b}$ & \begin{tabular}{|l|} 
Contaminate/ \\
damage the chilled \\
water or steam \\
supply system.
\end{tabular} & $\begin{array}{l}\text { Failure in heat exchanger } \\
\text { causes leak of process } \\
\text { material into chilled water } \\
\text { or steam supply. } \\
\text { Simulant mixture plugs } \\
\text { system. }\end{array}$ & -2 & 0 & 1 & $\begin{array}{l}\text { Condensate collection system and } \\
\text { blowdown on steam system } \\
\text { Periodic chemistry test on chilled } \\
\text { water system } \\
\text { Total Dissolved Solids (TDS) } \\
\text { monitoring on steam supply } \\
\text { Water chemistry maintenance }\end{array}$ & Operating procedures & $\begin{array}{l}\text { Contamination in the } \\
\text { system would take } \\
\text { significant time } \\
\text { before damage would } \\
\text { occur. }\end{array}$ & E0 & 3 \\
\hline PEP-30-4-02 & $4 \mathrm{~b}$ & $\begin{array}{l}\text { Contaminate/ } \\
\text { damage the chilled } \\
\text { water or steam } \\
\text { supply system. }\end{array}$ & $\begin{array}{l}\text { Failure of heat exchanger } \\
\text { causes leak of nitric acid } \\
\text { into chilled water supply } \\
\text { or steam supply. }\end{array}$ & -2 & 0 & 1 & $\begin{array}{l}\text { Condensate collection system and } \\
\text { blowdown on steam system } \\
\text { Periodic chemistry test on chilled } \\
\text { water system } \\
\text { TDS monitoring on steam supply } \\
\text { Water chemistry maintenance } \\
\end{array}$ & Operating procedures & $\begin{array}{l}\text { Contamination in the } \\
\text { system would take } \\
\text { significant time } \\
\text { before damage would } \\
\text { occur. }\end{array}$ & E0 & 3 \\
\hline PEP-30-4-03 & $4 \mathrm{~b}$ & Operational upset. & $\begin{array}{l}\text { High temperature in the } \\
\text { heating heat exchanger. } \\
\text { Lose flow of simulant } \\
\text { with steam in heat } \\
\text { exchanger. Dry out } \\
\text { system, depositing scale, } \\
\text { plugging ultrafilters. }\end{array}$ & & 0 & $\mathrm{n} / \mathrm{a}$ & \begin{tabular}{|l|} 
Flushing capability for system \\
Temperature monitoring \\
Pulse pots designed to clear ultrafilters \\
Operating procedures
\end{tabular} & $\mathrm{n} / \mathrm{a}$ & & E0 & 0 \\
\hline
\end{tabular}


Table E.1. Hazard Analysis Worksheets

\begin{tabular}{|c|c|c|c|c|c|c|c|c|c|c|c|}
\hline $\begin{array}{l}\text { Scenario } \\
\text { Number } \\
\end{array}$ & Node & $\begin{array}{c}\text { Hazardous } \\
\text { Condition } \\
\end{array}$ & Cause & $\mathbf{L}$ & C & $\begin{array}{c}\text { Risk } \\
\text { Score } \\
\end{array}$ & Potential Safeguard & $\begin{array}{l}\text { Credited } \\
\text { Safeguards }\end{array}$ & $\begin{array}{c}\text { Comments/ } \\
\text { Assumptions }\end{array}$ & $\mathbf{E}$ & $\$$ \\
\hline PEP-30-4-04 & $4 \mathrm{~b}$ & Operational upset. & $\begin{array}{l}\text { Low temperatures in the } \\
\text { cooling heat exchanger } \\
\text { due to stopping flow in } \\
\text { the process loop. } \\
\text { Precipitate oxalate and } \\
\text { other solids, plugging } \\
\text { ultrafilters. }\end{array}$ & & 0 & $\mathrm{n} / \mathrm{a}$ & \begin{tabular}{|l|} 
Flushing capability for system \\
Temperature monitoring \\
Pulse pots designed to clear ultrafilters \\
Operating procedures
\end{tabular} & $\mathrm{n} / \mathrm{a}$ & & E0 & 0 \\
\hline PEP-30-4-05 & $4 \mathrm{~b}$ & $\begin{array}{l}\text { Damage the heat } \\
\text { exchanger loop, } \\
\text { releasing heated } \\
\text { process material. }\end{array}$ & $\begin{array}{l}\text { Close isolation valves to } \\
\text { heat exchanger with } \\
\text { continued heating causes } \\
\text { over pressurization. }\end{array}$ & 1 & 5 & 6 & $\begin{array}{l}\text { Operating procedures } \\
\text { Pressure relief to heat exchanger } \\
\text { Pressure relief valve }\end{array}$ & $\begin{array}{l}\text { Operating procedures } \\
\text { PSV on heat } \\
\text { exchanger }\end{array}$ & $\begin{array}{l}\text { Relief valve added at } \\
\text { the } 90 \% \text { design. }\end{array}$ & E0 & $\mathrm{SD}$ \\
\hline PEP-30-4-06 & $4 \mathrm{~b}$ & $\begin{array}{l}\text { Large release of } \\
\text { steam to the } \\
\text { facility. }\end{array}$ & $\begin{array}{l}\text { Catastrophic failure of the } \\
\text { steam shell on the heat } \\
\text { exchanger results in large } \\
\text { release of steam. }\end{array}$ & -3 & 5 & 2 & Design of steam shell to code & $\begin{array}{l}\text { Design of steam shell } \\
\text { to code }\end{array}$ & & E0 & 3 \\
\hline PEP-30-4-07 & $4 \mathrm{~b}$ & $\begin{array}{l}\text { Collapse of steam } \\
\text { shell. }\end{array}$ & $\begin{array}{l}\text { Steam present in the shell, } \\
\text { blocked off. Vacuum } \\
\text { pulled collapsing shell on } \\
\text { cooling. }\end{array}$ & -1 & 0 & 2 & $\begin{array}{l}\text { Vacuum breakers on jacket } \\
\text { Operating procedures }\end{array}$ & Operating procedures & & E0 & 3 \\
\hline PEP-30-4-08 & $4 \mathrm{~b}$ & $\begin{array}{l}\text { Small release of } \\
\text { steam to the } \\
\text { facility. }\end{array}$ & \begin{tabular}{|l|} 
Structural failure of the \\
steam shell of exchanger. \\
Small leak to steam.
\end{tabular} & -1 & 2 & 1 & & $\begin{array}{l}\text { Design of steam shell } \\
\text { to code }\end{array}$ & & E0 & 1 \\
\hline PEP-30-4-09 & 4 & \begin{tabular}{|l|} 
Spill of simulant, \\
sodium hydroxide, \\
sodium \\
permanganate, \\
nitric acid, or \\
inhibited water.
\end{tabular} & $\begin{array}{l}\text { No flow in the } \\
\text { ultrafiltration loop due to } \\
\text { leak in chemical addition } \\
\text { or transfer piping (flanges, } \\
\text { etc.). }\end{array}$ & 0 & 4 & 4 & $\begin{array}{l}\text { Leak test at installation } \\
\text { Facility secondary containment } \\
\text { Operating procedures } \\
\text { PPE } \\
\text { Personnel training } \\
\text { Ultrafiltration feed tank level } \\
\text { detection (thermocouples, bubblers, } \\
\text { and laser) } \\
\text { Flow totalizers on major chemical } \\
\text { additions } \\
\text { Flow meter on transfer line }\end{array}$ & \begin{tabular}{|l} 
PPE \\
Facility secondary \\
containment \\
Operating procedures
\end{tabular} & $\begin{array}{l}\text { Potential for } \\
\text { caustic/acidic } \\
\text { burn/eye damage. } \\
\text { Leak is inside facility. }\end{array}$ & E0 & $\mathrm{SD}$ \\
\hline PEP-30-4-10 & 4 & Operational upset. & $\begin{array}{l}\text { Low pressure in system, } \\
\text { reducing filtration } \\
\text { capability of system. }\end{array}$ & & 0 & $\mathrm{n} / \mathrm{a}$ & & $\mathrm{n} / \mathrm{a}$ & & E0 & 0 \\
\hline PEP-30-4-11 & 4 & Operational upset. & \begin{tabular}{|l|} 
Backpulse filters when \\
pumps shut down. \\
Pushing $\sim 400$ psi air back \\
through system to UFP- \\
VSL-T02A. Air \\
generated aerosols that are
\end{tabular} & & 0 & $\mathrm{n} / \mathrm{a}$ & & $\mathrm{n} / \mathrm{a}$ & \begin{tabular}{|l|} 
Aerosols generated \\
will not exceed \\
environment permit \\
limits. \\
The pressure available \\
in air system changes \\
\end{tabular} & E0 & 0 \\
\hline
\end{tabular}


Table E.1. Hazard Analysis Worksheets

\begin{tabular}{|c|c|c|c|c|c|c|c|c|c|c|c|}
\hline $\begin{array}{l}\text { Scenario } \\
\text { Number }\end{array}$ & Node & $\begin{array}{c}\text { Hazardous } \\
\text { Condition }\end{array}$ & Cause & $\mathbf{L}$ & C & $\begin{array}{c}\text { Risk } \\
\text { Score }\end{array}$ & Potential Safeguard & $\begin{array}{c}\text { Credited } \\
\text { Safeguards }\end{array}$ & $\begin{array}{l}\text { Comments/ } \\
\text { Assumptions }\end{array}$ & $\mathbf{E}$ & $\$$ \\
\hline & & & $\begin{array}{l}\text { released through } \\
\text { ventilation. }\end{array}$ & & & & & & $\begin{array}{l}\text { from } 300 \text { psi to } 500 \\
\text { psi (controlled @ } \\
\text { 400psi) at } 90 \% \\
\text { design. }\end{array}$ & & \\
\hline PEP-30-4-12 & 4 & Operational upset. & $\begin{array}{l}\text { Low flow through ultra } \\
\text { filters plugs up filters, } \\
\text { reducing filtration } \\
\text { capabilities. }\end{array}$ & & 0 & $\mathrm{n} / \mathrm{a}$ & & $\mathrm{n} / \mathrm{a}$ & & E0 & 0 \\
\hline PEP-30-4-13 & 4 & $\begin{array}{l}\text { Loss of filtration } \\
\text { capability and } \\
\text { material in UFP- } \\
\text { VSL-62A/B. }\end{array}$ & $\begin{array}{l}\text { Failure in the ultrafilter } \\
\text { tube bundle results in } \\
\text { material backing up into } \\
\text { the pulse pots and } \\
\text { eventually to UFP-VSL- } \\
62 \mathrm{~A} / \mathrm{B} \text {. }\end{array}$ & 0 & 0 & 1 & Spare tubes on hand & None identified & & E0 & 1 \\
\hline PEP-30-4-14 & 4 & Operational upset. & $\begin{array}{l}\text { Temperature lowered } \\
\text { below leaching } \\
\text { temperature. Slows down } \\
\text { process. }\end{array}$ & & 0 & $\mathrm{n} / \mathrm{a}$ & & $\mathrm{n} / \mathrm{a}$ & & E0 & 0 \\
\hline PEP-30-4-15 & 4 & Operational upset. & $\begin{array}{l}\text { High temperature in } \\
\text { system results in loss of } \\
\text { suction and potential } \\
\text { pump damage. }\end{array}$ & & 0 & $\mathrm{n} / \mathrm{a}$ & & $\mathrm{n} / \mathrm{a}$ & & E0 & 0 \\
\hline PEP-30-4-17 & $4 \mathrm{a}$ & Operational upset. & $\begin{array}{l}\text { No flow through one } \\
\text { pulse pot due to plugging } \\
\text { of line or valve closure. } \\
\text { Eliminates capability to } \\
\text { pulse filter. }\end{array}$ & & 0 & $\mathrm{n} / \mathrm{a}$ & & $\mathrm{n} / \mathrm{a}$ & & E0 & 0 \\
\hline PEP-30-4-18 & $4 \mathrm{a}$ & Operational upset. & $\begin{array}{l}\text { Failure to shut off } \\
\sim 400 \text { psi air and do not } \\
\text { shut off valve at pulse pot. } \\
\text { Continue air flow across } \\
\text { filters, dry out utrafilters. } \\
\text { Potential to damage filter. }\end{array}$ & & 0 & $\mathrm{n} / \mathrm{a}$ & & $\mathrm{n} / \mathrm{a}$ & \begin{tabular}{|l|} 
The pressure available \\
in air system changes \\
from 300 psi to 500 \\
psi (controlled @ \\
400psi) at $90 \%$ \\
design. Need to \\
verify filter assembly \\
design pressure to \\
understand this event.
\end{tabular} & E0 & 0 \\
\hline PEP-30-4-19 & $4 \mathrm{a}$ & $\begin{array}{l}\text { Leak of permeate } \\
\text { and pressurized air } \\
\text { into facility. }\end{array}$ & $\begin{array}{l}\text { Catastrophic failure of } \\
\text { pulse pot structure. Event } \\
\text { happens when pulsing. }\end{array}$ & -3 & 5 & 2 & $\begin{array}{l}\text { Design of pulse pot to code } \\
\text { Pressure relief }\end{array}$ & $\begin{array}{l}\text { Design of pulse pot } \\
\text { to code } \\
\text { PSV on pulse pot }\end{array}$ & & E0 & SD \\
\hline
\end{tabular}


Table E.1. Hazard Analysis Worksheets

\begin{tabular}{|c|c|c|c|c|c|c|c|c|c|c|c|}
\hline $\begin{array}{l}\text { Scenario } \\
\text { Number }\end{array}$ & Node & $\begin{array}{l}\text { Hazardous } \\
\text { Condition }\end{array}$ & Cause & $\mathbf{L}$ & C & $\begin{array}{c}\text { Risk } \\
\text { Score }\end{array}$ & Potential Safeguard & $\begin{array}{c}\text { Credited } \\
\text { Safeguards }\end{array}$ & $\begin{array}{c}\text { Comments/ } \\
\text { Assumptions }\end{array}$ & $\mathbf{E}$ & $\$$ \\
\hline PEP-30-4-20 & $4 a$ & $\begin{array}{l}\text { Small pressurized } \\
\text { leak of permeate } \\
\text { to facility. }\end{array}$ & $\begin{array}{l}\text { Failure of pulse pot } \\
\text { structure, resulting in } \\
\text { small leak. }\end{array}$ & 0 & 3 & 3 & $\begin{array}{l}\text { Design of pulse pot to code } \\
\text { Pressure relief } \\
\text { When system is pressurized, the time } \\
\text { frame is short }\end{array}$ & $\begin{array}{l}\text { Design of pulse pot } \\
\text { to code }\end{array}$ & & E0 & SD \\
\hline PEP-30-4-21 & $4 a$ & $\begin{array}{l}\text { Release of reaction } \\
\text { aerosols out of } \\
\text { nitric acid tank to } \\
\text { facility. }\end{array}$ & $\begin{array}{l}\text { Backflow of permeate } \\
\text { back into the nitric acid } \\
\text { addition system. Reaction } \\
\text { in nitric acid tank } \\
\text { releasing aerosols out of } \\
\text { vent. }\end{array}$ & 0 & 3 & 3 & $\begin{array}{l}\text { Check valve on nitric system } \\
\text { Automatically controlled addition } \\
\text { valve } \\
\text { Automated addition sequence } \\
\text { Relief valve on pulse pot }\end{array}$ & Operating procedures & & E0 & SD \\
\hline PEP-90-4-01 & $4 \mathrm{a}$ & $\begin{array}{l}\text { Small leak of } \\
\text { simulant to } \\
\text { facility. }\end{array}$ & $\begin{array}{l}\text { No/low water in or no } \\
\text { flow from seal pots causes } \\
\text { loss of pump sealing. } \\
\text { Simulant leaks out of seal } \\
\text { into the facility. }\end{array}$ & 0 & 0 & 0 & & None identified & Added at $90 \%$ & E0 & 0 \\
\hline PEP-90-4-02 & $4 a$ & $\begin{array}{l}\text { Small leak of } \\
\text { simulant to } \\
\text { facility. }\end{array}$ & $\begin{array}{l}\text { High temperature in seal } \\
\text { pots causes damage to } \\
\text { pump seals. Simulant } \\
\text { leaks out of seal into the } \\
\text { facility. }\end{array}$ & 0 & 0 & 0 & & None identified & Added at $90 \%$ & E0 & 0 \\
\hline PEP-90-4-03 & $4 a$ & Operational upset. & $\begin{array}{l}\text { Overfill the seal pots } \\
\text { when adding water } \\
\text { (ventilation system off } \\
\text { and valve open). Spill } \\
\text { water to facility floor. } \\
\end{array}$ & & 0 & $\mathrm{n} / \mathrm{a}$ & & $\mathrm{n} / \mathrm{a}$ & Added at $90 \%$ & E0 & 0 \\
\hline PEP-90-4-04 & $4 a$ & $\begin{array}{l}\text { Small leak of } \\
\text { simulant to } \\
\text { facility. }\end{array}$ & $\begin{array}{l}\text { Low/no pressure from air } \\
\text { supply to seal pot system } \\
\text { causes loss of pump } \\
\text { sealing. Simulant leaks } \\
\text { out of seal into the } \\
\text { facility. }\end{array}$ & 0 & 0 & 0 & & None identified & Added at $90 \%$ & E0 & 0 \\
\hline PEP-90-4-05 & $4 \mathrm{a}$ & $\begin{array}{l}\text { Spill of simulant, } \\
\text { sodium hydroxide, } \\
\text { sodium } \\
\text { permanganate, } \\
\text { nitric acid, or } \\
\text { inhibited water. }\end{array}$ & $\begin{array}{l}\text { High pressure air causes } \\
\text { failure of filter assembly, } \\
\text { releasing material to the } \\
\text { facility. }\end{array}$ & -3 & 4 & 1 & TBD & $\begin{array}{l}\text { Design of filter } \\
\text { assembly }\end{array}$ & \begin{tabular}{|l|} 
Pumps on the tube \\
side can increase \\
pressure across filters
\end{tabular} & E0 & SD \\
\hline PEP-30-5-01 & 5 & Operational upset. & $\begin{array}{l}\text { No flow into the tank due } \\
\text { to closed isolation valve } \\
\text { or pump not operating. }\end{array}$ & & 0 & $\mathrm{n} / \mathrm{a}$ & & $\mathrm{n} / \mathrm{a}$ & & E0 & 0 \\
\hline
\end{tabular}


Table E.1. Hazard Analysis Worksheets

\begin{tabular}{|c|c|c|c|c|c|c|c|c|c|c|c|}
\hline $\begin{array}{l}\text { Scenario } \\
\text { Number }\end{array}$ & Node & $\begin{array}{l}\text { Hazardous } \\
\text { Condition }\end{array}$ & Cause & $\mathbf{L}$ & $\mathrm{C}$ & $\begin{array}{l}\text { Risk } \\
\text { Score }\end{array}$ & Potential Safeguard & $\begin{array}{l}\text { Credited } \\
\text { Safeguards }\end{array}$ & $\begin{array}{c}\text { Comments/ } \\
\text { Assumptions }\end{array}$ & $\mathbf{E}$ & $\$$ \\
\hline PEP-30-5-02 & 5 & $\begin{array}{l}\text { Spill of } \\
\text { concentrated } \\
\text { slurry, inhibited } \\
\text { water to the } \\
\text { facility floor. }\end{array}$ & $\begin{array}{l}\text { No flow into the tank due } \\
\text { to leak at the connection } \\
\text { to the flex hose } \\
\text { connection to the portable } \\
\text { pump. }\end{array}$ & 1 & 4 & 5 & $\begin{array}{l}\text { Facility secondary containment } \\
\text { Operating procedures } \\
\text { PPE } \\
\text { Personnel training } \\
\text { Tank level detection (bubbler) } \\
\text { Flow totalizer on inhibited water } \\
\end{array}$ & \begin{tabular}{|l|} 
PPE \\
Facility secondary \\
containment \\
Operating procedures \\
Slurry Concentrate \\
tank level detection \\
\end{tabular} & $\begin{array}{l}\text { Potential for caustic } \\
\text { burn/eye damage } \\
\mathrm{pH} \sim 12 .\end{array}$ & E1 & 1 \\
\hline PEP-30-5-03 & 5 & $\begin{array}{l}\text { Spill of } \\
\text { concentrated } \\
\text { slurry, inhibited } \\
\text { water. }\end{array}$ & $\begin{array}{l}\text { No flow into the tank due } \\
\text { to leak in transfer piping } \\
\text { (flanges, etc.). }\end{array}$ & 0 & 4 & 4 & $\begin{array}{l}\text { Leak test at installation } \\
\text { Facility secondary containment } \\
\text { Operating procedures } \\
\text { PPE } \\
\text { Personnel training } \\
\text { Tank level detection (bubbler) } \\
\text { Flow totalizer on inhibited water }\end{array}$ & \begin{tabular}{|l|} 
PPE \\
Facility secondary \\
containment \\
Operating procedures \\
Slurry Concentrate \\
tank level detection
\end{tabular} & $\begin{array}{l}\text { Potential for caustic } \\
\text { burn/eye damage } \\
\mathrm{pH} \sim 12 \text {. } \\
\text { Leak is inside facility. }\end{array}$ & E0 & 1 \\
\hline PEP-30-5-04 & 5 & Operational upset. & $\begin{array}{l}\text { No flow out of tank due to } \\
\text { portable pump not } \\
\text { operating. }\end{array}$ & & 0 & $\mathrm{n} / \mathrm{a}$ & & $\mathrm{n} / \mathrm{a}$ & & E0 & 0 \\
\hline PEP-30-5-05 & 5 & Operational upset. & $\begin{array}{l}\text { No flow out of tank due to } \\
\text { line plugging. }\end{array}$ & & 0 & $\mathrm{n} / \mathrm{a}$ & & $\mathrm{n} / \mathrm{a}$ & & E0 & 0 \\
\hline PEP-30-5-06 & 5 & $\begin{array}{l}\text { Spill of } \\
\text { concentrated } \\
\text { slurry, inhibited } \\
\text { water. }\end{array}$ & $\begin{array}{l}\text { Structural integrity of tank } \\
\text { degraded, resulting in } \\
\text { leak. }\end{array}$ & -3 & 4 & 1 & Design of tanks to code & $\begin{array}{l}\text { Design of Slurry } \\
\text { Concentrate tank to } \\
\text { code }\end{array}$ & $\begin{array}{l}\text { Spill to floor of } \\
\text { facility. } \\
\text { Potential for caustic } \\
\text { burn/eye damage } \\
\text { pH 14. } \\
\text { Likelihood assigned } \\
\text { based on tank to code. } \\
304 \text { SST is listed as } \\
\text { having excellent } \\
\text { chemical } \\
\text { compatibility to nitric } \\
\text { acid, and good } \\
\text { compatibility with } \\
\text { NaOH, and oxalic } \\
\text { acid. }\end{array}$ & E0 & $\mathrm{SD}$ \\
\hline PEP-30-5-07 & 5 & $\begin{array}{l}\text { Spill of } \\
\text { concentrated } \\
\text { slurry, inhibited } \\
\text { water. }\end{array}$ & $\begin{array}{l}\text { No flow to the receiving } \\
\text { end due to leak in transfer } \\
\text { piping/pumps. }\end{array}$ & 0 & 4 & 4 & $\begin{array}{l}\text { Leak test at installation } \\
\text { Facility secondary containment } \\
\text { Operating procedures } \\
\text { PPE } \\
\text { Personnel training } \\
\text { Tank level detection (bubbler) }\end{array}$ & \begin{tabular}{|l|} 
PPE \\
Facility secondary \\
containment \\
Operating procedures \\
Slurry Concentrate \\
tank level detection \\
\end{tabular} & $\begin{array}{l}\text { Spill to floor of } \\
\text { facility. } \\
\text { Potential for caustic } \\
\text { burn/eye damage } \\
\text { pH 12. }\end{array}$ & E0 & $\mathrm{SD}$ \\
\hline
\end{tabular}


Table E.1. Hazard Analysis Worksheets

\begin{tabular}{|c|c|c|c|c|c|c|c|c|c|c|c|}
\hline $\begin{array}{l}\text { Scenario } \\
\text { Number }\end{array}$ & Node & $\begin{array}{l}\text { Hazardous } \\
\text { Condition }\end{array}$ & Cause & $\mathbf{L}$ & C & $\begin{array}{l}\text { Risk } \\
\text { Score } \\
\end{array}$ & Potential Safeguard & $\begin{array}{l}\text { Credited } \\
\text { Safeguards }\end{array}$ & $\begin{array}{c}\text { Comments/ } \\
\text { Assumptions }\end{array}$ & $\mathbf{E}$ & $\$$ \\
\hline PEP-30-5-08 & 5 & $\begin{array}{l}\text { Spill of } \\
\text { concentrated } \\
\text { slurry, inhibited } \\
\text { water. }\end{array}$ & $\begin{array}{l}\text { No flow to receiving end } \\
\text { due to leak at the } \\
\text { connection to the simulant } \\
\text { addition flex hose. }\end{array}$ & 1 & 4 & 5 & $\begin{array}{l}\text { Facility secondary containment } \\
\text { Operating procedures } \\
\text { PPE } \\
\text { Personnel training } \\
\text { Tank level detection (bubbler) }\end{array}$ & \begin{tabular}{|l|} 
PPE \\
Facility secondary \\
containment \\
Operating procedures \\
Slurry Concentrate \\
tank level detection \\
\end{tabular} & $\begin{array}{l}\text { Spill to floor of } \\
\text { facility. } \\
\text { Potential for caustic } \\
\text { burn/eye damage } \\
\text { pH 12. }\end{array}$ & E0 & 1 \\
\hline PEP-30-5-09 & 5 & $\begin{array}{l}\text { No issue } \\
\text { identified. }\end{array}$ & Filling tank too fast. & & 0 & $\mathrm{n} / \mathrm{a}$ & & $\mathrm{n} / \mathrm{a}$ & $\begin{array}{l}\text { Ventilation system is } \\
\text { designed to } \\
\text { accommodate for any } \\
\text { excess aerosols } \\
\text { generated during this } \\
\text { event. }\end{array}$ & E0 & 0 \\
\hline PEP-30-5-10 & 5 & $\begin{array}{l}\text { Overflow of tank } \\
\text { contents to } \\
\text { ventilation system, } \\
\text { damaging fans, } \\
\text { exchanger, and } \\
\text { blower. Boil off } \\
\text { of liquids forming } \\
\text { solids in vent } \\
\text { lines. }\end{array}$ & $\begin{array}{l}\text { Too much material added } \\
\text { to the tank, resulting } \\
\text { overflow of tank to the } \\
\text { ventilation system. }\end{array}$ & 1 & 0 & 3 & $\begin{array}{l}\text { Facility secondary containment } \\
\text { Operational procedures } \\
\text { Tank level detection (bubbler) } \\
\text { High level alarm } \\
\text { Flow totalizer on inhibited water } \\
\text { system } \\
\text { Interlock to shutdown inhibited water }\end{array}$ & $\begin{array}{l}\text { Slurry concentrate } \\
\text { tank level detection } \\
\text { and alarm }\end{array}$ & & E2 & 2 \\
\hline PEP-30-5-11 & 5 & \begin{tabular}{|l|} 
Spill of \\
concentrated \\
slurry, inhibited \\
water on to facility \\
floor.
\end{tabular} & $\begin{array}{l}\text { Too much material added } \\
\text { to the tank with valves } \\
\text { open, resulting in spill out } \\
\text { of transfer line onto floor. }\end{array}$ & 0 & 4 & 4 & $\begin{array}{l}\text { Facility secondary containment } \\
\text { Operational procedures } \\
\text { Tank level detection (bubbler) } \\
\text { High level alarm } \\
\text { Flow totalizer on inhibited water } \\
\text { system } \\
\text { Interlock to shutdown inhibited water }\end{array}$ & \begin{tabular}{|l|} 
PPE \\
Facility secondary \\
containment \\
Operating procedures \\
Slurry concentrate \\
tank level detection \\
and alarm \\
\end{tabular} & & E0 & SD \\
\hline PEP-30-5-12 & 5 & Operational upset. & $\begin{array}{l}\text { Too much material added } \\
\text { to the tank with valves } \\
\text { open, resulting in } \\
\text { backflow to UFB-VSL- } \\
62 \mathrm{~A} . \\
\end{array}$ & & 0 & $\mathrm{n} / \mathrm{a}$ & & $\mathrm{n} / \mathrm{a}$ & & E0 & 0 \\
\hline PEP-30-5-13 & 5 & \begin{tabular}{|l} 
Spill of \\
concentrated \\
slurry, inhibited \\
water, nitric acid, \\
or simulant on to \\
facility floor.
\end{tabular} & \begin{tabular}{|l|} 
No flow to tank due to \\
portable pump discharge \\
line open, transferring \\
material directly out open \\
valve.
\end{tabular} & 1 & 4 & 5 & $\begin{array}{l}\text { Facility secondary containment } \\
\text { Operating procedures } \\
\text { Level detection on intended source } \\
\text { tank } \\
\text { Level detection on intended receiving } \\
\text { tank }\end{array}$ & \begin{tabular}{|l|} 
PPE \\
Facility secondary \\
containment \\
Operating procedures \\
Slurry Concentrate \\
tank level detection \\
\end{tabular} & & E0 & SD \\
\hline PEP-30-5-14 & 5 & Operational upset. & $\begin{array}{l}\text { Too much material added } \\
\text { to the tank. High level in } \\
\text { tank. }\end{array}$ & 1 & 0 & 3 & $\begin{array}{l}\text { Tank Level detection (bubbler) } \\
\text { Flow totalizer } \\
\text { Operating procedures }\end{array}$ & $\begin{array}{l}\text { Slurry Concentrate } \\
\text { tank level detection } \\
\text { and alarm } \\
\end{array}$ & & E0 & 2 \\
\hline
\end{tabular}


Table E.1. Hazard Analysis Worksheets

\begin{tabular}{|c|c|c|c|c|c|c|c|c|c|c|c|}
\hline $\begin{array}{l}\text { Scenario } \\
\text { Number }\end{array}$ & Node & $\begin{array}{l}\text { Hazardous } \\
\text { Condition }\end{array}$ & Cause & $\mathbf{L}$ & C & $\begin{array}{l}\text { Risk } \\
\text { Score }\end{array}$ & Potential Safeguard & $\begin{array}{l}\text { Credited } \\
\text { Safeguards }\end{array}$ & $\begin{array}{l}\text { Comments/ } \\
\text { Assumptions }\end{array}$ & $\mathbf{E}$ & $\$$ \\
\hline PEP-30-5-15 & 5 & $\begin{array}{l}\text { Overflow of } \\
\text { receipt tank } \\
\text { contents to } \\
\text { ventilation system } \\
\text { damaging fans, } \\
\text { exchanger, and } \\
\text { blower. Boil off } \\
\text { of liquids forming } \\
\text { solids in vent } \\
\text { lines. }\end{array}$ & \begin{tabular}{|l|} 
Backflow of material \\
from UFP-VSL-T62A to \\
tank T27 caused by valves \\
open and over fill T62A.
\end{tabular} & 0 & 0 & 2 & $\begin{array}{l}\text { Level detection in T27 and alarms } \\
\text { Operating procedures }\end{array}$ & Operating procedures & & E2 & 2 \\
\hline PEP-30-5-16 & 5 & Operational upset. & $\begin{array}{l}\text { Low pressure in receipt } \\
\text { tank due to plugging of } \\
\text { ventilation (closure of } \\
\text { damper) during transfer } \\
\text { out. }\end{array}$ & & 0 & $\mathrm{n} / \mathrm{a}$ & $\begin{array}{l}\text { Design of tanks to } 7.5 \text { psid external } \\
\text { Stop or bore through on the damper to } \\
\text { prevent full closure. }\end{array}$ & $\mathrm{n} / \mathrm{a}$ & $\begin{array}{l}\text { System is designed } \\
\text { such that the pumps } \\
\text { cannot draw enough } \\
\text { to damage tank. }\end{array}$ & E0 & 0 \\
\hline PEP-30-5-18 & 5 & $\begin{array}{l}\text { Failure of tank and } \\
\text { spill tank contents. }\end{array}$ & $\begin{array}{l}\text { High pressure in receipt } \\
\text { tank due to ventilation } \\
\text { blocked (closure of } \\
\text { damper) and overfilling of } \\
\text { tank. }\end{array}$ & 1 & 5 & 6 & $\begin{array}{l}\text { Pressure relief valve } \\
\text { Local pressure indicator for tank } \\
\text { Operating procedures } \\
\text { Pressure Transmitter } \\
\text { Stops or bore through on the damper } \\
\text { to prevent full closure. }\end{array}$ & \begin{tabular}{|l|} 
PSV on Slurry \\
Concentrate tank \\
Stop or bore through \\
on the damper to \\
prevent full closure.
\end{tabular} & $\begin{array}{l}\text { Spill to floor of } \\
\text { facility. }\end{array}$ & E0 & SD \\
\hline PEP-30-5-19 & 5 & Operational upset. & $\begin{array}{l}\text { Agitator in receipt tank } \\
\text { does not operate or poor } \\
\text { mixing due to VFD too } \\
\text { slow. Solids settle out. } \\
\end{array}$ & & 0 & $\mathrm{n} / \mathrm{a}$ & & $\mathrm{n} / \mathrm{a}$ & & E0 & 0 \\
\hline PEP-30-5-20 & 5 & Operational upset. & $\begin{array}{l}\text { Prolonged settling in tank } \\
\text { causes accumulation of } \\
\text { solids in tank and results } \\
\text { in difficulty moving/ } \\
\text { mixing of material. }\end{array}$ & & 0 & $\mathrm{n} / \mathrm{a}$ & Operating procedures & $\mathrm{n} / \mathrm{a}$ & & E0 & 0 \\
\hline PEP-30-6-01 & $6 a$ & Operational upset. & $\begin{array}{l}\text { High temperature in the } \\
\text { tank prevents emptying } \\
\text { the tank. Affects } \\
\text { operation time. }\end{array}$ & & 0 & $\mathrm{n} / \mathrm{a}$ & & $\mathrm{n} / \mathrm{a}$ & & E0 & 0 \\
\hline PEP-30-6-02 & $6 a$ & Operational upset. & \begin{tabular}{|l} 
No flow into the tank due \\
to closed isolation valve \\
or pump not operating.
\end{tabular} & & 0 & $\mathrm{n} / \mathrm{a}$ & & $\mathrm{n} / \mathrm{a}$ & & E0 & 0 \\
\hline
\end{tabular}


Table E.1. Hazard Analysis Worksheets

\begin{tabular}{|c|c|c|c|c|c|c|c|c|c|c|c|}
\hline $\begin{array}{l}\text { Scenario } \\
\text { Number } \\
\end{array}$ & Node & $\begin{array}{c}\text { Hazardous } \\
\text { Condition }\end{array}$ & Cause & $\mathbf{L}$ & C & $\begin{array}{l}\text { Risk } \\
\text { Score } \\
\end{array}$ & Potential Safeguard & $\begin{array}{l}\text { Credited } \\
\text { Safeguards }\end{array}$ & $\begin{array}{c}\text { Comments/ } \\
\text { Assumptions }\end{array}$ & $\mathbf{E}$ & $\$$ \\
\hline PEP-30-6-03 & $6 a$ & $\begin{array}{l}\text { Spill of permeate, } \\
\text { concentrated } \\
\text { slurry, simulant, } \\
\text { sodium hydroxide, } \\
\text { sodium } \\
\text { permanganate, } \\
\text { nitric acid, or } \\
\text { inhibited water to } \\
\text { the facility floor. }\end{array}$ & $\begin{array}{l}\text { No flow into the tank due } \\
\text { to leak at the connection } \\
\text { to the flex hose } \\
\text { connection to the portable } \\
\text { pump. }\end{array}$ & 1 & 4 & 5 & $\begin{array}{l}\text { Facility secondary containment } \\
\text { Operating procedures } \\
\text { PPE } \\
\text { Personnel training } \\
\text { Evaporator tank level detection } \\
\text { (bubbler) } \\
\text { Flow totalizer on inhibited water } \\
\text { Flow totalizer on sodium hydroxide }\end{array}$ & \begin{tabular}{|l|} 
PPE \\
Facility secondary \\
containment \\
Operating procedures \\
Permeate receipt tank \\
level detection
\end{tabular} & $\begin{array}{l}\text { Potential for } \\
\text { caustic/acidic } \\
\text { burn/eye damage. }\end{array}$ & E0 & 1 \\
\hline PEP-30-6-04 & $6 a$ & $\begin{array}{l}\text { Spill of permeate, } \\
\text { concentrated } \\
\text { slurry, simulant, } \\
\text { sodium hydroxide, } \\
\text { sodium } \\
\text { permanganate, } \\
\text { nitric acid, or } \\
\text { inhibited water to } \\
\text { the facility floor. } \\
\end{array}$ & $\begin{array}{l}\text { No flow into the tank due } \\
\text { to leak in transfer piping } \\
\text { (flanges, etc.). }\end{array}$ & 0 & 4 & 4 & $\begin{array}{l}\text { Leak test at installation } \\
\text { Facility secondary containment } \\
\text { Operating procedures } \\
\text { PPE } \\
\text { Personnel training } \\
\text { Evaporator tank level detection } \\
\text { (bubbler) } \\
\text { Flow totalizer on inhibited water } \\
\text { Flow totalizer on sodium hydroxide } \\
\end{array}$ & \begin{tabular}{|l|} 
PPE \\
Facility secondary \\
containment \\
Operating procedures \\
Permeate receipt tank \\
level detection
\end{tabular} & $\begin{array}{l}\text { Potential for caustic } \\
\text { burn/eye damage } \\
\mathrm{pH} \sim 12 \text {. } \\
\text { Leak is inside facility. }\end{array}$ & E0 & 1 \\
\hline PEP-30-6-05 & $6 a$ & Operational upset. & $\begin{array}{l}\text { No flow out of tank due to } \\
\text { portable pump not } \\
\text { operating. }\end{array}$ & & 0 & $\mathrm{n} / \mathrm{a}$ & & $\mathrm{n} / \mathrm{a}$ & & E0 & 0 \\
\hline PEP-30-6-06 & $6 a$ & Operational upset. & $\begin{array}{l}\text { No flow out of tank due to } \\
\text { line plugging. }\end{array}$ & & 0 & $\mathrm{n} / \mathrm{a}$ & & $\mathrm{n} / \mathrm{a}$ & & E0 & 0 \\
\hline PEP-30-6-07 & $6 a$ & $\begin{array}{l}\text { Spill of permeate, } \\
\text { concentrated } \\
\text { slurry, simulant, } \\
\text { sodium hydroxide, } \\
\text { sodium } \\
\text { permanganate, } \\
\text { nitric acid, or } \\
\text { inhibited water to } \\
\text { the facility floor. }\end{array}$ & $\begin{array}{l}\text { Structural integrity of tank } \\
\text { degraded resulting in leak. }\end{array}$ & -3 & 4 & 1 & Design of tanks to code & $\begin{array}{l}\text { Design of permeate } \\
\text { receipt tanks to code }\end{array}$ & $\begin{array}{l}\text { Spill to floor of } \\
\text { facility. } \\
\text { Potential for caustic } \\
\text { burn/eye damage } \\
\text { pH 14. } \\
\text { Likelihood assigned } \\
\text { based on tank to code. } \\
304 \text { SST is listed as } \\
\text { having excellent } \\
\text { chemical } \\
\text { compatibility to nitric } \\
\text { acid, and good } \\
\text { compatibility with } \\
\text { NaOH, and oxalic } \\
\text { acid. } \\
\end{array}$ & E0 & SD \\
\hline PEP-30-6-08 & $6 a$ & $\begin{array}{l}\text { Spill of permeate, } \\
\text { concentrated } \\
\text { slurry, simulant, } \\
\text { sodium hydroxide, }\end{array}$ & $\begin{array}{l}\text { No flow to the receiving } \\
\text { tank due to leak in } \\
\text { transfer piping/pumps. }\end{array}$ & 0 & 4 & 4 & $\begin{array}{l}\text { Leak test at installation } \\
\text { Facility secondary containment } \\
\text { Operating procedures } \\
\text { PPE }\end{array}$ & \begin{tabular}{|l|} 
PPE \\
Facility secondary \\
containment \\
Operating procedures
\end{tabular} & $\begin{array}{l}\text { Spill to floor of } \\
\text { facility. } \\
\text { Potential for caustic }\end{array}$ & E0 & $\mathrm{SD}$ \\
\hline
\end{tabular}


Table E.1. Hazard Analysis Worksheets

\begin{tabular}{|c|c|c|c|c|c|c|c|c|c|c|c|}
\hline $\begin{array}{l}\text { Scenario } \\
\text { Number }\end{array}$ & Node & $\begin{array}{c}\text { Hazardous } \\
\text { Condition }\end{array}$ & Cause & $\mathbf{L}$ & $\mathrm{C}$ & $\begin{array}{l}\text { Risk } \\
\text { Score }\end{array}$ & Potential Safeguard & $\begin{array}{c}\text { Credited } \\
\text { Safeguards }\end{array}$ & $\begin{array}{c}\text { Comments/ } \\
\text { Assumptions }\end{array}$ & $\mathbf{E}$ & $\$$ \\
\hline & & $\begin{array}{l}\text { sodium } \\
\text { permanganate, } \\
\text { nitric acid, or } \\
\text { inhibited water to } \\
\text { the facility floor. }\end{array}$ & & & & & $\begin{array}{l}\text { Personnel training } \\
\text { Evaporator tank and receiving tank } \\
\text { level detection (bubbler) }\end{array}$ & $\begin{array}{l}\text { Permeate receipt tank } \\
\text { level detection }\end{array}$ & $\begin{array}{l}\text { burn/eye damage } \\
\mathrm{pH} \sim 12 .\end{array}$ & & \\
\hline PEP-30-6-09 & $6 a$ & \begin{tabular}{|l|} 
Spill of permeate, \\
concentrated \\
slurry, simulant, \\
sodium hydroxide, \\
sodium \\
permanganate, \\
nitric acid, or \\
inhibited water to \\
the facility floor. \\
\end{tabular} & $\begin{array}{l}\text { No flow to receiving end } \\
\text { due to leak at the } \\
\text { connection to the simulant } \\
\text { addition flex hose. }\end{array}$ & 1 & 4 & 5 & $\begin{array}{l}\text { Facility secondary containment } \\
\text { Operating procedures } \\
\text { PPE } \\
\text { Personnel training } \\
\text { Evaporator tank level detection } \\
\text { (bubbler) }\end{array}$ & \begin{tabular}{|l|} 
PPE \\
Facility secondary \\
containment \\
Operating procedures \\
Permeate receipt tank \\
level detection
\end{tabular} & $\begin{array}{l}\text { Spill to floor of } \\
\text { facility. } \\
\text { Potential for caustic } \\
\text { burn/eye damage } \\
\text { pH 12. }\end{array}$ & E0 & 1 \\
\hline PEP-30-6-10 & $6 a$ & $\begin{array}{l}\text { No issue } \\
\text { identified. }\end{array}$ & Filling tank too fast. & & 0 & $\mathrm{n} / \mathrm{a}$ & & $\mathrm{n} / \mathrm{a}$ & $\begin{array}{l}\text { Ventilation system is } \\
\text { designed to } \\
\text { accommodate for any } \\
\text { excess aerosols } \\
\text { generated during this } \\
\text { event. }\end{array}$ & E0 & 0 \\
\hline PEP-30-6-11 & $6 \mathrm{a}$ & $\begin{array}{l}\text { Overflow of tank } \\
\text { contents to } \\
\text { ventilation system, } \\
\text { damaging fans, } \\
\text { exchanger and } \\
\text { blower. Boiling } \\
\text { off liquids forming } \\
\text { solids in vent } \\
\text { lines. }\end{array}$ & $\begin{array}{l}\text { Too much material added } \\
\text { to the tank resulting } \\
\text { overflow of tank to the } \\
\text { ventilation system. }\end{array}$ & 1 & 0 & 3 & $\begin{array}{l}\text { Facility secondary containment } \\
\text { Operating procedures } \\
\text { Tank level detection (bubbler) } \\
\text { High level alarm } \\
\text { Flow totalizer on inhibited water } \\
\text { system } \\
\text { Interlock to shutdown inhibited water }\end{array}$ & $\begin{array}{l}\text { Permeate receipt tank } \\
\text { level detection and } \\
\text { alarm }\end{array}$ & & E2 & 2 \\
\hline PEP-30-6-12 & $6 a$ & \begin{tabular}{|l|} 
Spill of permeate, \\
concentrated \\
slurry, simulant, \\
sodium hydroxide, \\
sodium \\
permanganate, \\
nitric acid, or \\
inhibited water to \\
the facility floor. \\
\end{tabular} & $\begin{array}{l}\text { Too much material added } \\
\text { to the tank with valves } \\
\text { open, resulting in spill out } \\
\text { of transfer line onto floor. }\end{array}$ & 0 & 4 & 4 & $\begin{array}{l}\text { Facility secondary containment } \\
\text { Operating procedures } \\
\text { Evaporator tank level detection } \\
\text { (bubbler) } \\
\text { High level alarm } \\
\text { Flow totalizer on inhibited water } \\
\text { system } \\
\text { Interlock to shutdown inhibited water }\end{array}$ & \begin{tabular}{|l} 
PPE \\
Facility secondary \\
containment \\
Operating procedures \\
Permeate receipt tank \\
level detection and \\
alarm
\end{tabular} & & E0 & $\mathrm{SD}$ \\
\hline
\end{tabular}


Table E.1. Hazard Analysis Worksheets

\begin{tabular}{|c|c|c|c|c|c|c|c|c|c|c|c|}
\hline $\begin{array}{l}\text { Scenario } \\
\text { Number }\end{array}$ & Node & $\begin{array}{l}\text { Hazardous } \\
\text { Condition }\end{array}$ & Cause & $\mathbf{L}$ & C & $\begin{array}{l}\text { Risk } \\
\text { Score }\end{array}$ & Potential Safeguard & $\begin{array}{l}\text { Credited } \\
\text { Safeguards }\end{array}$ & $\begin{array}{c}\text { Comments/ } \\
\text { Assumptions }\end{array}$ & $\mathbf{E}$ & $\$$ \\
\hline PEP-30-6-13 & $6 \mathrm{a}$ & Operational upset. & $\begin{array}{l}\text { Too much material added } \\
\text { to the tank with valves } \\
\text { open, resulting in } \\
\text { backflow to other process } \\
\text { tanks. }\end{array}$ & & 0 & $\mathrm{n} / \mathrm{a}$ & & $\mathrm{n} / \mathrm{a}$ & & E0 & 0 \\
\hline PEP-30-6-14 & $6 a$ & $\begin{array}{l}\text { Spill of permeate, } \\
\text { concentrated } \\
\text { slurry, simulant, } \\
\text { sodium hydroxide, } \\
\text { sodium } \\
\text { permanganate, } \\
\text { nitric acid, or } \\
\text { inhibited water to } \\
\text { the facility floor } \\
\text { from the chemical } \\
\text { addition tank. }\end{array}$ & $\begin{array}{l}\text { Too much material added } \\
\text { to the tank with valves } \\
\text { open, resulting in } \\
\text { backflow to chemical } \\
\text { addition tank (sodium } \\
\text { hydroxide, inhibited } \\
\text { water). }\end{array}$ & -1 & 4 & 3 & $\begin{array}{l}\text { Facility secondary containment } \\
\text { Operating procedures } \\
\text { Evaporator tank level detection } \\
\text { (bubbler) } \\
\text { High level alarm } \\
\text { Automatic block valve on sodium } \\
\text { hydroxide line } \\
\text { Check valves }\end{array}$ & Operating procedures & & E0 & SD \\
\hline PEP-30-6-15 & $6 a$ & $\begin{array}{l}\text { Spill of permeate, } \\
\text { concentrated } \\
\text { slurry, simulant, } \\
\text { sodium hydroxide, } \\
\text { sodium } \\
\text { permanganate, } \\
\text { nitric acid, or } \\
\text { inhibited water to } \\
\text { the facility floor. }\end{array}$ & \begin{tabular}{|l|} 
No flow to receiving tank \\
due to portable pump \\
discharge line open \\
transferring material \\
directly out open valve.
\end{tabular} & 1 & 4 & 5 & $\begin{array}{l}\text { Facility secondary containment } \\
\text { Operating procedures } \\
\text { Level detection on intended source } \\
\text { tank } \\
\text { Level detection on intended receiving } \\
\text { tank }\end{array}$ & \begin{tabular}{|l|} 
PPE \\
Facility secondary \\
containment \\
Operating procedures \\
Permeate receipt tank \\
level detection
\end{tabular} & & E0 & SD \\
\hline PEP-30-6-16 & $6 a$ & Operational upset. & $\begin{array}{l}\text { Too much material added } \\
\text { to the tank. High level in } \\
\text { tank. }\end{array}$ & & 0 & $\mathrm{n} / \mathrm{a}$ & $\begin{array}{l}\text { Tank Level detection (bubbler) } \\
\text { Flow totalizer } \\
\text { Operating procedures }\end{array}$ & $\mathrm{n} / \mathrm{a}$ & & E0 & SD \\
\hline PEP-30-6-17 & $6 a$ & $\begin{array}{l}\text { Overflow of } \\
\text { receipt tank } \\
\text { contents to } \\
\text { ventilation system, } \\
\text { damaging fans, } \\
\text { exchanger and } \\
\text { blower. Boiling } \\
\text { off liquids and } \\
\text { forming solids in } \\
\text { vent lines. }\end{array}$ & $\begin{array}{l}\text { Misroute of material from } \\
\text { source vessel to tank } \\
\text { T62A caused by valve } \\
\text { misalignment (intended to } \\
\text { transfer to tank other than } \\
\text { T62A). Overflow T62A. }\end{array}$ & 1 & 0 & 3 & $\begin{array}{l}\text { Level detection in } \mathrm{T} 62 \mathrm{~A} \text { and alarms } \\
\text { Operating procedures }\end{array}$ & Operating procedures & & E2 & 2 \\
\hline
\end{tabular}


Table E.1. Hazard Analysis Worksheets

\begin{tabular}{|c|c|c|c|c|c|c|c|c|c|c|c|}
\hline $\begin{array}{l}\text { Scenario } \\
\text { Number }\end{array}$ & Node & $\begin{array}{l}\text { Hazardous } \\
\text { Condition }\end{array}$ & Cause & $\mathbf{L}$ & C & $\begin{array}{l}\text { Risk } \\
\text { Score } \\
\end{array}$ & Potential Safeguard & $\begin{array}{l}\text { Credited } \\
\text { Safeguards }\end{array}$ & $\begin{array}{c}\text { Comments/ } \\
\text { Assumptions }\end{array}$ & $\mathbf{E}$ & $\$$ \\
\hline PEP-30-6-18 & $6 a$ & Operational upset. & $\begin{array}{l}\text { Low pressure in receipt } \\
\text { tank due to plugging of } \\
\text { ventilation (closure of } \\
\text { damper) during transfer } \\
\text { out. }\end{array}$ & & 0 & $\mathrm{n} / \mathrm{a}$ & $\begin{array}{l}\text { Design of tanks to } 7.5 \text { psid external } \\
\text { Stop or bore through on the damper to } \\
\text { prevent full closure. }\end{array}$ & $\mathrm{n} / \mathrm{a}$ & & E0 & 0 \\
\hline PEP-30-6-20 & $6 a$ & \begin{tabular}{|l|} 
Spill of permeate, \\
concentrated \\
slurry, simulant, \\
sodium hydroxide, \\
sodium \\
permanganate, \\
nitric acid, or \\
inhibited water to \\
the facility floor. \\
\end{tabular} & $\begin{array}{l}\text { High pressure in receipt } \\
\text { tank due to ventilation } \\
\text { blocked (closure of } \\
\text { damper) and overfilling of } \\
\text { tank. }\end{array}$ & 1 & 5 & 6 & $\begin{array}{l}\text { Pressure relief valve } \\
\text { Local pressure indicator for } \\
\text { evaporator tank } \\
\text { High level alarm in evaporator tank } \\
\text { Operating procedures } \\
\text { Pressure Transmitter } \\
\text { Stops or bore through on the damper } \\
\text { to prevent full closure. }\end{array}$ & $\begin{array}{l}\text { PSV on permeate } \\
\text { receipt tank } \\
\text { Stop or bore through } \\
\text { on the damper to } \\
\text { prevent full closure. }\end{array}$ & $\begin{array}{l}\text { Spill to floor of } \\
\text { facility. }\end{array}$ & E0 & SD \\
\hline PEP-30-6-21 & $6 a$ & Operational upset. & $\begin{array}{l}\text { Agitator in receipt tank } \\
\text { does not operate or poor } \\
\text { mixing due to VFD too } \\
\text { slow. Solids settle out. } \\
\end{array}$ & & 0 & $\mathrm{n} / \mathrm{a}$ & & $\mathrm{n} / \mathrm{a}$ & & E0 & 0 \\
\hline PEP-30-6-22 & $6 a$ & Operational upset. & \begin{tabular}{|l|} 
Prolonged settling in tank \\
causes accumulation of \\
solids in tank and results \\
in difficulty moving/ \\
mixing of material. \\
\end{tabular} & & 0 & $\mathrm{n} / \mathrm{a}$ & Operating procedures & $\mathrm{n} / \mathrm{a}$ & & E0 & 0 \\
\hline PEP-30-6-23 & $6 a$ & $\begin{array}{l}\text { Damage } \\
\text { equipment and } \\
\text { holdup of } \\
\text { operation. }\end{array}$ & \begin{tabular}{|l|} 
Evaporate material to \\
higher concentration than \\
wanted. Let tank cool; \\
material solidifies in tank. \\
Potential to damage \\
agitator.
\end{tabular} & 1 & 0 & 5 & $\begin{array}{l}\text { Evaporator tank level detection } \\
\text { (bubbler) } \\
\text { Sampling } \\
\text { Operating procedures }\end{array}$ & \begin{tabular}{|l|} 
Operating procedures \\
Permeate evaporation \\
tank level detection \\
Permeate evaporation \\
tank temperature \\
indication
\end{tabular} & & E0 & 4 \\
\hline PEP-30-6-24 & $6 a$ & \begin{tabular}{|l|} 
Failure of tank \\
releasing \\
permeate, \\
concentrated \\
slurry, simulant, \\
sodium hydroxide, \\
sodium \\
permanganate, \\
nitric acid, or \\
inhibited water to \\
the facility.
\end{tabular} & $\begin{array}{l}\text { Over heat evaporator tank } \\
\text { with ventilation blocked } \\
\text { (closure of damper, } \\
\text { plugging) causes over } \\
\text { pressurization and damage } \\
\text { to tank. }\end{array}$ & 1 & 5 & 6 & $\begin{array}{l}\text { Pressure relief valve } \\
\text { Local pressure indicator for tank } \\
\text { Temperature indication } \\
\text { High-high temperature shutdown } \\
\text { Operating procedures } \\
\text { Pressure Transmitter } \\
\text { Stops or bore through on the damper } \\
\text { to prevent full closure. }\end{array}$ & $\begin{array}{l}\text { PSV on permeate } \\
\text { receipt tank } \\
\text { Stop or bore through } \\
\text { on the damper to } \\
\text { prevent full closure. }\end{array}$ & & E0 & SD \\
\hline
\end{tabular}


Table E.1. Hazard Analysis Worksheets

\begin{tabular}{|c|c|c|c|c|c|c|c|c|c|c|c|}
\hline $\begin{array}{l}\text { Scenario } \\
\text { Number }\end{array}$ & Node & $\begin{array}{c}\text { Hazardous } \\
\text { Condition }\end{array}$ & Cause & $\mathbf{L}$ & C & $\begin{array}{c}\text { Risk } \\
\text { Score } \\
\end{array}$ & Potential Safeguard & $\begin{array}{c}\text { Credited } \\
\text { Safeguards } \\
\end{array}$ & $\begin{array}{c}\text { Comments/ } \\
\text { Assumptions }\end{array}$ & E & $\$$ \\
\hline PEP-90-6-02 & $6 \mathrm{a}$ & Operational upset. & $\begin{array}{l}\text { Low temperature in the } \\
\text { steam jacket. Cannot heat } \\
\text { collection tank. }\end{array}$ & & 0 & $\mathrm{n} / \mathrm{a}$ & & $\mathrm{n} / \mathrm{a}$ & & E0 & 0 \\
\hline PEP-90-6-03 & $6 \mathrm{a}$ & $\begin{array}{l}\text { Small release of } \\
\text { steam to the } \\
\text { facility. }\end{array}$ & \begin{tabular}{|l|} 
Structural failure of the \\
steam jacket on collection \\
tank. Small leak of steam.
\end{tabular} & -1 & 2 & 1 & Pressure relief valve & $\begin{array}{l}\text { Design of steam } \\
\text { jacket }\end{array}$ & & E0 & 1 \\
\hline PEP-90-6-04 & $6 \mathrm{a}$ & $\begin{array}{l}\text { Large release of } \\
\text { steam to the } \\
\text { facility. }\end{array}$ & $\begin{array}{l}\text { Catastrophic failure of the } \\
\text { steam jacket on collection } \\
\text { tank results in large } \\
\text { release of steam. }\end{array}$ & -3 & 5 & 2 & $\begin{array}{l}\text { Design of steam jacket to code } \\
\text { Pressure relief valve }\end{array}$ & $\begin{array}{l}\text { Design of steam } \\
\text { jacket }\end{array}$ & & E0 & 3 \\
\hline PEP-90-6-05 & $6 \mathrm{a}$ & $\begin{array}{l}\text { Collapse of steam } \\
\text { jacket. }\end{array}$ & \begin{tabular}{|l|} 
Steam present in the \\
steam jacket on the \\
collection tank. Block off \\
jacket; add cold water to \\
refill. Vacuum pulled \\
collapsing jacket.
\end{tabular} & -1 & 0 & 2 & $\begin{array}{l}\text { Vacuum breakers on jacket } \\
\text { Operating procedures }\end{array}$ & $\begin{array}{l}\text { Vacuum breaker on } \\
\text { steam jacket }\end{array}$ & & E0 & 3 \\
\hline PEP-90-6-06 & $6 \mathrm{a}$ & $\begin{array}{l}\text { Small release of } \\
\text { steam to the } \\
\text { facility. }\end{array}$ & $\begin{array}{l}\text { Failure of flex hose } \\
\text { connection from steam } \\
\text { jacket to steam supply } \\
\text { system (steam line or } \\
\text { traps). Release steam to } \\
\text { the facility. }\end{array}$ & -1 & 2 & 1 & & None identified & & E0 & 1 \\
\hline PEP-90-6-01 & $6 \mathrm{a}$ & \begin{tabular}{|l|} 
Overflow of tank \\
contents to \\
ventilation system, \\
damaging fans, \\
exchanger, and \\
blower. Boiling \\
off liquids forming \\
solids in vent \\
lines. \\
\end{tabular} & \begin{tabular}{|l|} 
Leak or failure of cooling \\
line inside permeate \\
receipt tank resulting in \\
tank overflow into the \\
vessel ventilation system. \\
\end{tabular} & 1 & 0 & 3 & $\begin{array}{l}\text { Permeate collection tank level } \\
\text { detection (bubbler) } \\
\text { High level alarm } \\
\text { Chiller tank low level alarm }\end{array}$ & $\begin{array}{l}\text { Permeate receipt tank } \\
\text { level detection and } \\
\text { alarm }\end{array}$ & Added at $90 \%$ & E2 & 2 \\
\hline PEP-90-7-01 & 7 & \begin{tabular}{|l|} 
Process \\
aerosols/vapors in \\
manned space \\
atmosphere \\
(caustic, aluminum \\
salts, and \\
chromium).
\end{tabular} & $\begin{array}{l}\text { Blower failed (loss of } \\
\text { power) or breaks in } \\
\text { system upstream of } \\
\text { blower. Any process } \\
\text { vessel (T01A/B and } \\
\text { T02A) in operation vents } \\
\text { to the manned facility } \\
\text { spaces. }\end{array}$ & 0 & 2 & 2 & Pressure transmitters on tank systems & None identified & & E0 & 0 \\
\hline PEP-90-7-02 & 7 & \begin{tabular}{|l|} 
Process \\
aerosols/vapors in \\
manned space
\end{tabular} & $\begin{array}{l}\text { No flow in the system } \\
\text { (damper closed). Any } \\
\text { process vessel (T01A/B }\end{array}$ & 0 & 2 & 2 & $\begin{array}{l}\text { Design feature - dampers have holes } \\
\text { in them so they cannot completely } \\
\text { stop airflow }\end{array}$ & None identified & & E0 & 0 \\
\hline
\end{tabular}


Table E.1. Hazard Analysis Worksheets

\begin{tabular}{|c|c|c|c|c|c|c|c|c|c|c|c|}
\hline $\begin{array}{l}\text { Scenario } \\
\text { Number }\end{array}$ & Node & $\begin{array}{c}\text { Hazardous } \\
\text { Condition }\end{array}$ & Cause & $\mathbf{L}$ & C & $\begin{array}{l}\text { Risk } \\
\text { Score }\end{array}$ & Potential Safeguard & $\begin{array}{l}\text { Credited } \\
\text { Safeguards }\end{array}$ & $\begin{array}{c}\text { Comments/ } \\
\text { Assumptions }\end{array}$ & $\mathbf{E}$ & $\$$ \\
\hline & & \begin{tabular}{|l|} 
atmosphere \\
(caustic, aluminum \\
salts, and \\
chromium).
\end{tabular} & $\begin{array}{l}\text { and T02A) in operation } \\
\text { vents to the manned } \\
\text { facility spaces. }\end{array}$ & & & & & & & & \\
\hline PEP-30-7-03 & 7 & \begin{tabular}{|l|} 
Process \\
aerosols/vapors in \\
manned space \\
atmosphere \\
(caustic, aluminum \\
salts, and \\
chromium).
\end{tabular} & $\begin{array}{l}\text { Low temperature due to } \\
\text { failure in heat exchange } \\
\text { system or low/no steam } \\
\text { flow. Cold ambient } \\
\text { outside temperatures. } \\
\text { Condensate collects in the } \\
\text { suction and discharge of } \\
\text { blower. Causes blower to } \\
\text { shut down. Any process } \\
\text { vessel (T01A/B and } \\
\text { T02A) in operation vents } \\
\text { to the manned facility } \\
\text { spaces. }\end{array}$ & -2 & 2 & 0 & $\begin{array}{l}\text { Temperature indicator on heat } \\
\text { exchanger air discharge } \\
\text { Pressure transmitters on tank systems } \\
\text { Steam trace on vent line }\end{array}$ & None identified & \begin{tabular}{|l|} 
This event was re- \\
evaluated at $90 \%$ and \\
likelihood and \\
consequences applied.
\end{tabular} & E0 & 0 \\
\hline PEP-90-7-03 & 7 & \begin{tabular}{|l|} 
Process \\
aerosols/vapors in \\
manned space \\
atmosphere \\
(caustic, aluminum \\
salts, and \\
chromium).
\end{tabular} & $\begin{array}{l}\text { Low temperature due to } \\
\text { failure in heat exchange } \\
\text { system or low/no steam } \\
\text { flow. Cold ambient } \\
\text { outside temperatures. } \\
\text { Condensate collects in the } \\
\text { knock-out pot and blocks } \\
\text { the vent inlet. Shuts down } \\
\text { ventilation flow. Any } \\
\text { process vessel (T01A/B } \\
\text { and T02A) in operation } \\
\text { vents to the manned } \\
\text { facility spaces. }\end{array}$ & -1 & 2 & 1 & $\begin{array}{l}\text { Level indication on knock out pot } \\
\text { Steam trace on lines } \\
\text { Temperature indicator on knock out } \\
\text { pot } \\
\text { Pressure transmitters on tanks }\end{array}$ & None identified & & E0 & 0 \\
\hline PEP-30-7-05 & 7 & \begin{tabular}{|l|} 
Process \\
aerosols/vapors in \\
manned space \\
atmosphere \\
(caustic, aluminum \\
salts, and \\
chromium).
\end{tabular} & $\begin{array}{l}\text { Failure of steam coil in } \\
\text { knock-out pot results in } \\
\text { direct release of steam to } \\
\text { blower. Affects the } \\
\text { blower's capability to } \\
\text { provide adequate } \\
\text { ventilation to process } \\
\text { systems. Any process } \\
\text { vessel (T01A/B and } \\
\text { T02A) in operation vents } \\
\text { to the manned facility } \\
\text { spaces. }\end{array}$ & -2 & 2 & 0 & $\begin{array}{l}\text { Temperature indicator on knock out } \\
\text { pot } \\
\text { Pressure transmitters on tank systems }\end{array}$ & None identified & \begin{tabular}{|l|} 
These event was \\
evaluated at $90 \%$ and \\
likelihood and \\
consequences applied. \\
Modifications were \\
made to reflect \\
wording more specific \\
to $90 \%$ design.
\end{tabular} & E0 & 0 \\
\hline
\end{tabular}


Table E.1. Hazard Analysis Worksheets

\begin{tabular}{|c|c|c|c|c|c|c|c|c|c|c|c|}
\hline $\begin{array}{l}\text { Scenario } \\
\text { Number }\end{array}$ & Node & $\begin{array}{l}\text { Hazardous } \\
\text { Condition }\end{array}$ & Cause & $\mathbf{L}$ & C & $\begin{array}{l}\text { Risk } \\
\text { Score }\end{array}$ & Potential Safeguard & $\begin{array}{l}\text { Credited } \\
\text { Safeguards }\end{array}$ & $\begin{array}{c}\text { Comments/ } \\
\text { Assumptions }\end{array}$ & $\mathbf{E}$ & $\$$ \\
\hline PEP-30-8-01 & 8 & Operational upset. & $\begin{array}{l}\text { DI system not operating; } \\
\text { add contamination into } \\
\text { steam system. Damage to } \\
\text { boiler. }\end{array}$ & & 0 & $\mathrm{n} / \mathrm{a}$ & & $\mathrm{n} / \mathrm{a}$ & & E0 & 0 \\
\hline PEP-30-8-02 & 8 & Operational upset. & \begin{tabular}{|l|} 
Shutdown of process cuts \\
steam supply to process. \\
High pressure left in \\
system. Steam released \\
through pressure relief \\
valves.
\end{tabular} & & 0 & $\mathrm{n} / \mathrm{a}$ & & $\mathrm{n} / \mathrm{a}$ & & E0 & 0 \\
\hline PEP-90-8-01 & 8 & $\begin{array}{l}\text { Large release of } \\
\text { steam outside and } \\
\text { possible natural } \\
\text { gas fire. }\end{array}$ & $\begin{array}{l}\text { Structural failure of the } \\
\text { boiler. Release steam and } \\
\text { supply gas to outside. }\end{array}$ & -2 & 5 & 3 & Design of boiler to code - ASME 1 & Design boiler to code & Added at $90 \%$ & E0 & 4 \\
\hline PEP-90-8-02 & 8 & $\begin{array}{l}\text { Large release of } \\
\text { steam outside and } \\
\text { possible natural } \\
\text { gas fire. }\end{array}$ & \begin{tabular}{|l|} 
Overpressure in boiler \\
results in structural \\
failure. Release steam \\
and supply gas to outside.
\end{tabular} & -1 & 5 & 4 & $\begin{array}{l}\text { Pressure safety valve } \\
\text { Pressure regulating valve }\end{array}$ & PSV on boiler & Added at $90 \%$ & E0 & 4 \\
\hline PEP-30-8-04 & 8 & $\begin{array}{l}\text { Large release of } \\
\text { steam in manned } \\
\text { area. }\end{array}$ & $\begin{array}{l}\text { Catastrophic failure of } \\
\text { system within the facility. }\end{array}$ & -3 & 5 & 2 & $\begin{array}{l}\text { Operating procedures } \\
\text { Pressure relief }\end{array}$ & $\begin{array}{l}\text { Design of steam } \\
\text { system to code } \\
\text { Operating procedures }\end{array}$ & $\begin{array}{l}\text { The likelihood of the } \\
\text { event considers that } \\
\text { the system is designed } \\
\text { to code. }\end{array}$ & E0 & SD \\
\hline PEP-30-8-05 & 8 & $\begin{array}{l}\text { Small release of } \\
\text { steam in manned } \\
\text { area. }\end{array}$ & $\begin{array}{l}\text { Minor failure of system } \\
\text { within the facility. }\end{array}$ & 0 & 3 & 3 & $\begin{array}{l}\text { Operating procedures } \\
\text { Pressure relief }\end{array}$ & $\begin{array}{l}\text { Design of steam } \\
\text { system to code }\end{array}$ & & E0 & SD \\
\hline PEP-90-8-03 & 8 & $\begin{array}{l}\text { Release of boiling } \\
\text { water or steam } \\
\text { outside. }\end{array}$ & $\begin{array}{l}\text { Feed water temperature } \\
\text { control failure results in } \\
\text { high temperature in feed } \\
\text { water tank. Water boils } \\
\text { and releases out of tank } \\
\text { overflow. } \\
\end{array}$ & -1 & 1 & 0 & & None identified & & E0 & 0 \\
\hline PEP-90-8-04 & 8 & $\begin{array}{l}\text { Natural gas fire } \\
\text { outside facility. }\end{array}$ & $\begin{array}{l}\text { Failure of boiler natural } \\
\text { gas supply results in fire. } \\
\text { Damage to facility } \\
\text { structure. }\end{array}$ & -3 & 3 & 0 & $\begin{array}{l}\text { System is designed to code } \\
\text { Shutoff valve in gas supply and at } \\
\text { boiler }\end{array}$ & $\begin{array}{l}\text { Natural gas system is } \\
\text { designed to code }\end{array}$ & & E0 & 2 \\
\hline PEP-FN-8-01 & 8 & $\begin{array}{l}\text { Natural gas fire } \\
\text { outside facility. }\end{array}$ & $\begin{array}{l}\text { Natural gas supply line } \\
\text { damaged by impact with } \\
\text { load/vehicle resulting in } \\
\text { fire. }\end{array}$ & -1 & 3 & 2 & $\begin{array}{l}\text { Bollards around gas connection } \\
\text { Line is attached to building } \\
\text { Raised pad }\end{array}$ & $\begin{array}{l}\text { Bollards around gas } \\
\text { connection }\end{array}$ & & E0 & 2 \\
\hline
\end{tabular}


Table E.1. Hazard Analysis Worksheets

\begin{tabular}{|c|c|c|c|c|c|c|c|c|c|c|c|}
\hline $\begin{array}{l}\text { Scenario } \\
\text { Number }\end{array}$ & Node & $\begin{array}{l}\text { Hazardous } \\
\text { Condition }\end{array}$ & Cause & $\mathbf{L}$ & C & $\begin{array}{c}\text { Risk } \\
\text { Score }\end{array}$ & Potential Safeguard & $\begin{array}{c}\text { Credited } \\
\text { Safeguards }\end{array}$ & $\begin{array}{l}\text { Comments/ } \\
\text { Assumptions }\end{array}$ & $\mathbf{E}$ & $\$$ \\
\hline PEP-30-8-06 & 8 & Operational upset. & $\begin{array}{l}\text { Condensate trap blow } \\
\text { through, condensate not } \\
\text { captured in trap. } \\
\text { Condensate returns to } \\
\text { steam system. } \\
\text { Condensate tank heats up. } \\
\text { System becomes less } \\
\text { efficient. }\end{array}$ & & 0 & $\mathrm{n} / \mathrm{a}$ & & $\mathrm{n} / \mathrm{a}$ & & E0 & 0 \\
\hline PEP-30-9-01 & 9 & $\begin{array}{l}\text { Incomplete/“out of } \\
\text { spec” batch of } \\
\text { material. Lost } \\
\text { time for test. }\end{array}$ & $\begin{array}{l}\text { No flow, low total, or } \\
\text { high total, from SHR- } \\
\text { VSL-T02 results in out of } \\
\text { specification caustic. }\end{array}$ & 1 & 0 & 3 & \begin{tabular}{|l|} 
Operating procedures \\
Level detection on SHR--VSL-T01 \\
Flow totalizer from SHR-VSL-T02
\end{tabular} & None identified & & E0 & 2 \\
\hline PEP-30-9-02 & 9 & $\begin{array}{l}\text { Incomplete/“out of } \\
\text { spec” batch of } \\
\text { material. Lost } \\
\text { time for test. }\end{array}$ & $\begin{array}{l}\text { Wrong material brought } \\
\text { in to SHR-VSL-T02 } \\
\text { results in out of } \\
\text { specification caustic. }\end{array}$ & 1 & 0 & 3 & Vendor certification of deliveries & Operating procedures & & E0 & 2 \\
\hline PEP-30-9-03 & 9 & $\begin{array}{l}\text { Incomplete/“out of } \\
\text { spec” batch of } \\
\text { material. Lost } \\
\text { time for test. }\end{array}$ & $\begin{array}{l}\text { Wrong volume of DI } \\
\text { water results in out of } \\
\text { specification of caustic. }\end{array}$ & 1 & 0 & 3 & \begin{tabular}{|l|} 
Operating procedures \\
Level detection on SHR-VSL-T01 \\
Flow totalizer from SHR-VSL-T02
\end{tabular} & None identified & & E0 & 2 \\
\hline PEP-30-9-04 & 9 & $\begin{array}{l}\text { Spill of chemical } \\
\text { to facility. }\end{array}$ & $\begin{array}{l}\text { Plug vent on chemical } \\
\text { addition tank while } \\
\text { transferring material in or } \\
\text { out. Create vacuum or } \\
\text { overpressurization on } \\
\text { tank, damaging tank. }\end{array}$ & -1 & 3 & 2 & $\begin{array}{l}\text { Design of tanks to } 7.5 \text { psid external } \\
\text { Cleaning ports on knock out pots }\end{array}$ & None identified & & E0 & SD \\
\hline PEP-30-9-05 & 9 & $\begin{array}{l}\text { Spill of chemical } \\
\text { to facility. }\end{array}$ & Overfill the tank. & 1 & 3 & 4 & $\begin{array}{l}\text { Operating procedures } \\
\text { Level detection on SHR--VSL-T01 } \\
\text { High level alarm } \\
\text { Drip pan } \\
\text { Overflow drum }\end{array}$ & $\begin{array}{l}\text { Chemical tank level } \\
\text { detection and alarm }\end{array}$ & \begin{tabular}{|l|} 
Added drip pan under \\
acid tank and the 19M \\
NaOH tank at $90 \%$ \\
design. \\
Overflow drum added \\
to catch overflow on \\
Final HA
\end{tabular} & E0 & SD \\
\hline PEP-30-9-06 & 9 & Operational upset. & $\begin{array}{l}\text { Low temperature due to } \\
\text { low ambient temperatures } \\
\text { outside, no heating in the } \\
\text { facility. Change viscosity } \\
\text { of caustic (solidifies). }\end{array}$ & & 0 & $\mathrm{n} / \mathrm{a}$ & Heat trace on 19 Molar & $\mathrm{n} / \mathrm{a}$ & & E0 & 0 \\
\hline
\end{tabular}


Table E.1. Hazard Analysis Worksheets

\begin{tabular}{|c|c|c|c|c|c|c|c|c|c|c|c|}
\hline $\begin{array}{l}\text { Scenario } \\
\text { Number }\end{array}$ & Node & $\begin{array}{l}\text { Hazardous } \\
\text { Condition }\end{array}$ & Cause & $\mathbf{L}$ & C & $\begin{array}{l}\text { Risk } \\
\text { Score }\end{array}$ & Potential Safeguard & $\begin{array}{l}\text { Credited } \\
\text { Safeguards }\end{array}$ & $\begin{array}{l}\text { Comments/ } \\
\text { Assumptions }\end{array}$ & $\mathbf{E}$ & $\$$ \\
\hline PEP-30-9-07 & 9 & $\begin{array}{l}\text { Spill of chemical } \\
\text { to the facility. }\end{array}$ & $\begin{array}{l}\text { Failure of SHR-VSL-T01 } \\
\text { (2 M caustic tank). }\end{array}$ & -3 & 4 & 1 & & $\begin{array}{l}\text { Design of chemical } \\
\text { tanks }\end{array}$ & $\begin{array}{l}\text { Tanks are designed to } \\
\text { meet environment but } \\
\text { they are not code- } \\
\text { stamped. } \\
\text { The likelihood of the } \\
\text { event considers that } \\
\text { the system is designed } \\
\text { to code. }\end{array}$ & E0 & SD \\
\hline PEP-FN-9-01 & 9 & $\begin{array}{l}\text { Spray of } 19 \mathrm{M} \\
\text { caustic to the } \\
\text { facility }\end{array}$ & \begin{tabular}{|l|} 
Small line failure \\
downstream of caustic \\
metering pump in the RO \\
system.
\end{tabular} & -1 & 2 & 1 & Operator rounds (visual identification) & $\begin{array}{l}\begin{array}{l}\text { Operator rounds } \\
\text { (visual identification) }\end{array} \\
\end{array}$ & & E0 & SD \\
\hline PEP-FN-9-02 & 9 & Operational upset & $\begin{array}{l}\text { RO pump provides higher } \\
\text { than expected pressure to } \\
\text { system; ruptures line } \\
\text { between pump and } \\
\text { membrane. Leak water to } \\
\text { facility. }\end{array}$ & & 0 & $\mathrm{n} / \mathrm{a}$ & & $\mathrm{n} / \mathrm{a}$ & $\begin{array}{l}\text { Pumps run } 500 \text { psi } \\
\text { high flow/900 psi low } \\
\text { flow }\end{array}$ & E0 & SD \\
\hline PEP-90-10-01 & 10 & Operational upset. & \begin{tabular}{|l|} 
Temperature of chilled \\
water too high from \\
chilled water tank. \\
Degrades heat exchanger \\
performance.
\end{tabular} & & 0 & $\mathrm{n} / \mathrm{a}$ & & $\mathrm{n} / \mathrm{a}$ & & E0 & 0 \\
\hline PEP-90-10-02 & 10 & Operational upset. & $\begin{array}{l}\text { Temperatures of chilled } \\
\text { water too low in chilled } \\
\text { water tank due to low } \\
\text { ambient temperatures. } \\
\text { Chiller pump shuts down } \\
\text { (T02). }\end{array}$ & & 0 & $\mathrm{n} / \mathrm{a}$ & $\begin{array}{l}\text { Tank Insulation } \\
\text { Continual recirculation of system } \\
\text { through pumps } \\
\text { Glycol in water }\end{array}$ & $\mathrm{n} / \mathrm{a}$ & & E0 & 0 \\
\hline PEP-90-10-03 & 10 & $\begin{array}{l}\text { Release of } \\
\text { water/glycol to the } \\
\text { environment. }\end{array}$ & $\begin{array}{l}\text { Structural failure in chiller } \\
\text { water system dumps water } \\
\text { and glycol onto ground, } \\
\text { outside facility. }\end{array}$ & -1 & 0 & -1 & & None identified & & E1 & 0 \\
\hline PEP-90-10-04 & 10 & $\begin{array}{l}\text { Release of } \\
\text { water/glycol to the } \\
\text { environment. }\end{array}$ & $\begin{array}{l}\text { Overfill the chilled water } \\
\text { tank during water addition } \\
\text { (manual addition). } \\
\text { Overflow to the ground, } \\
\text { outside the facility. }\end{array}$ & 0 & 0 & 0 & $\begin{array}{l}\text { Level transmitter and local level } \\
\text { indicator }\end{array}$ & None identified & & E1 & 0 \\
\hline PEP-90-10-05 & 10 & Operational upset. & $\begin{array}{l}\text { Low/no water in chilled } \\
\text { water tank. Lose chilling } \\
\text { capability. }\end{array}$ & & 0 & $\mathrm{n} / \mathrm{a}$ & & $\mathrm{n} / \mathrm{a}$ & & E0 & \\
\hline
\end{tabular}


Table E.1. Hazard Analysis Worksheets

\begin{tabular}{|c|c|c|c|c|c|c|c|c|c|c|c|}
\hline $\begin{array}{l}\text { Scenario } \\
\text { Number }\end{array}$ & Node & $\begin{array}{c}\text { Hazardous } \\
\text { Condition }\end{array}$ & Cause & $\mathbf{L}$ & C & $\begin{array}{l}\text { Risk } \\
\text { Score }\end{array}$ & Potential Safeguard & $\begin{array}{c}\text { Credited } \\
\text { Safeguards } \\
\end{array}$ & $\begin{array}{c}\text { Comments/ } \\
\text { Assumptions }\end{array}$ & $\mathbf{E}$ & $\$$ \\
\hline PEP-90-10-06 & 10 & Operational upset. & $\begin{array}{l}\text { Do not replenish glycol } \\
\text { into chilled water tank } \\
\text { when refilling water. } \\
\text { Low ambient } \\
\text { temperatures in system } \\
\text { causes freezing in system. } \\
\text { Lose chilling capabilities } \\
\text { and damage system. }\end{array}$ & & 0 & $\mathrm{n} / \mathrm{a}$ & & $\mathrm{n} / \mathrm{a}$ & & E0 & 0 \\
\hline $\begin{array}{l}\text { PEP-FN-10- } \\
01\end{array}$ & 10 & Operational upset. & $\begin{array}{l}\text { Bypass on chilled water } \\
\text { system loop valved to } \\
\text { recirculate when } \\
\text { unintended. Degrades } \\
\text { heat exchanger function } \\
\text { or loose cooling } \\
\text { capabilities. Potential to } \\
\text { damage system. }\end{array}$ & & 0 & $\mathrm{n} / \mathrm{a}$ & & $\mathrm{n} / \mathrm{a}$ & & E0 & 0 \\
\hline PEP-30-11-01 & 11 & Operational upset. & $\begin{array}{l}\text { No/low flow in } \\
\text { compressed air system } \\
\text { due to loss of instrument } \\
\text { air. Lose operation of } \\
\text { control valves, PJMs, etc. } \\
\text { Shutdown operation in } \\
\text { fail safe configuration. }\end{array}$ & & 0 & $\mathrm{n} / \mathrm{a}$ & & $\mathrm{n} / \mathrm{a}$ & & E0 & 0 \\
\hline PEP-30-11-02 & 11 & Operational upset. & $\begin{array}{l}\text { Contamination in the } \\
\text { instrument air (excess } \\
\text { moisture). Affects } \\
\text { operation of air operated } \\
\text { equipment. Degraded } \\
\text { equipment reliability. }\end{array}$ & & 0 & $\mathrm{n} / \mathrm{a}$ & Drier system instrumentation & $\mathrm{n} / \mathrm{a}$ & & E0 & 0 \\
\hline PEP-30-11-03 & 11 & Operational upset. & $\begin{array}{l}\text { Fail to close the manual } \\
\text { valve during operation of } \\
\text { the pulse pot. } \\
\text { Accumulator has failed, } \\
\text { so there is not the } \\
\text { necessary pressure in the } \\
\text { pulse pot. When system } \\
\text { is pulsed (quick open } \\
\text { valve), the permeate } \\
\text { backflows into the } \\
\text { instrument air system } \\
\text { back to the accumulator. }\end{array}$ & & 0 & $\mathrm{n} / \mathrm{a}$ & Pressure indicator on the pulse pot & $\mathrm{n} / \mathrm{a}$ & & E0 & 0 \\
\hline
\end{tabular}


Table E.1. Hazard Analysis Worksheets

\begin{tabular}{|c|c|c|c|c|c|c|c|c|c|c|c|}
\hline $\begin{array}{l}\text { Scenario } \\
\text { Number }\end{array}$ & Node & $\begin{array}{l}\text { Hazardous } \\
\text { Condition }\end{array}$ & Cause & $\mathbf{L}$ & C & $\begin{array}{c}\text { Risk } \\
\text { Score } \\
\end{array}$ & Potential Safeguard & $\begin{array}{l}\text { Credited } \\
\text { Safeguards }\end{array}$ & $\begin{array}{l}\text { Comments/ } \\
\text { Assumptions }\end{array}$ & $\mathbf{E}$ & $\$$ \\
\hline PEP-30-11-04 & 11 & $\begin{array}{l}\text { Personnel injury } \\
\text { due to debris } \\
\text { projectiles. }\end{array}$ & $\begin{array}{l}\text { Failure of the air } \\
\text { compression system } \\
\text { outside of facility. }\end{array}$ & -3 & 4 & 1 & $\begin{array}{l}\text { Design of relief valve on accumulator } \\
\text { to code }\end{array}$ & $\begin{array}{l}\text { Accumulator } \\
\text { designed to code }\end{array}$ & $\begin{array}{l}\text { The compressor, } \\
\text { amplifier and } \\
\text { accumulator are } \\
\text { located in the yard } \\
\text { between the East and } \\
\text { West facilities. }\end{array}$ & E0 & $\mathrm{SD}$ \\
\hline PEP-90-11-01 & 11 & $\begin{array}{l}\text { Personnel injury } \\
\text { due to debris } \\
\text { projectiles. }\end{array}$ & $\begin{array}{l}\text { Overpressure in pulse pot } \\
\text { compressed air system } \\
\text { due to amplifier pressures } \\
\text { exceeding design pressure } \\
(482 \text { psi). }\end{array}$ & -2 & 5 & 3 & $\begin{array}{l}\text { Pressure safety valve downstream of } \\
\text { receiver } \\
\text { Pressure regulating valve downstream } \\
\text { of receiver }\end{array}$ & $\begin{array}{l}\text { PSV on pulse pots } \\
\text { PSV on compressed } \\
\text { air system }\end{array}$ & $\begin{array}{l}\text { The system is } \\
\text { expected to operate at } \\
400 \text { psi but the } \\
\text { amplifier can generate } \\
\text { up to } 500 \text { psi. }\end{array}$ & E0 & SD \\
\hline PEP-30-12-01 & 12 & $\begin{array}{l}\text { Carryover of } \\
\text { material to the } \\
\text { ventilation system. }\end{array}$ & $\begin{array}{l}\text { No/low flow (failure) in } \\
\text { vacuum system. PJMs are } \\
\text { less effective; results in } \\
\text { overblow. }\end{array}$ & 1 & 0 & 3 & $\begin{array}{l}\text { PLC } \\
\text { PJM Level Detector } \\
\text { PJM Pressure Detector } \\
\text { PJM Rack Air Supply Valve }\end{array}$ & \begin{tabular}{|l|} 
PLC \\
PJM Level Detector \\
PJM Pressure \\
Detector \\
PJM Rack Air \\
Supply Valve \\
\end{tabular} & $\begin{array}{l}\text { Revised at } 90 \% \text { to } \\
\text { reflect PJM overblow } \\
\text { controls }\end{array}$ & E1 & 2 \\
\hline PEP-30-12-02 & 12 & Operational upset. & $\begin{array}{l}\text { Use of vacuum system on } \\
\text { PJMs in heated tanks. } \\
\text { Elevated temperatures at } \\
\text { PJM causes flashing of } \\
\text { material when vacuum } \\
\text { initiated. Vapors entering } \\
\text { into the vacuum system } \\
\text { go over the barometric leg } \\
\text { and into the system, } \\
\text { eventually reaching } \\
\text { vacuum pump. Damage } \\
\text { pump. }\end{array}$ & & 0 & $\mathrm{n} / \mathrm{a}$ & Cyclone separator & $\mathrm{n} / \mathrm{a}$ & & E0 & 0 \\
\hline PEP-30-12-03 & 12 & $\begin{array}{l}\text { Carryover of } \\
\text { material to the } \\
\text { ventilation system. }\end{array}$ & $\begin{array}{l}\text { Contamination/ } \\
\text { particulates in the vacuum } \\
\text { air system due to the } \\
\text { cyclone separator not } \\
\text { operating as expected. } \\
\text { Plug valves causing } \\
\text { system to be less } \\
\text { effective. Affects } \\
\text { operation of PJMs, } \\
\text { resulting in overblow. }\end{array}$ & 1 & 0 & 3 & $\begin{array}{l}\text { PLC } \\
\text { PJM Level Detector } \\
\text { PJM Pressure Detector } \\
\text { PJM Rack Air Supply Valve }\end{array}$ & $\begin{array}{l}\text { PLC } \\
\text { PJM Level Detector } \\
\text { PJM Pressure } \\
\text { Detector } \\
\text { PJM Rack Air } \\
\text { Supply Valve }\end{array}$ & $\begin{array}{l}\text { Revised at } 90 \% \text { to } \\
\text { reflect PJM overblow } \\
\text { controls }\end{array}$ & E1 & 2 \\
\hline
\end{tabular}


Table E.1. Hazard Analysis Worksheets

\begin{tabular}{|c|c|c|c|c|c|c|c|c|c|c|c|}
\hline $\begin{array}{l}\text { Scenario } \\
\text { Number }\end{array}$ & Node & $\begin{array}{c}\text { Hazardous } \\
\text { Condition } \\
\end{array}$ & Cause & $\mathbf{L}$ & C & $\begin{array}{l}\text { Risk } \\
\text { Score } \\
\end{array}$ & Potential Safeguard & $\begin{array}{c}\text { Credited } \\
\text { Safeguards } \\
\end{array}$ & $\begin{array}{c}\text { Comments/ } \\
\text { Assumptions }\end{array}$ & E & $\$$ \\
\hline PEP-90-12-01 & 12 & Operational upset. & $\begin{array}{l}\text { Vacuum is higher than } \\
\text { expected, pulling material } \\
\text { from the PJMs over the } \\
\text { barometric leg and into } \\
\text { system, eventually } \\
\text { reaching vacuum pump. } \\
\text { Damage pump. }\end{array}$ & & 0 & $\mathrm{n} / \mathrm{a}$ & & $\mathrm{n} / \mathrm{a}$ & & E0 & 0 \\
\hline PEP-90-12-02 & 12 & Operational upset. & $\begin{array}{l}\text { Loss of cooling to the heat } \\
\text { exchanger. Lose vacuum } \\
\text { capabilities. }\end{array}$ & & 0 & $\mathrm{n} / \mathrm{a}$ & & $\mathrm{n} / \mathrm{a}$ & & E0 & 0 \\
\hline PEP-90-12-03 & 12 & \begin{tabular}{|l|} 
Carryover of \\
material to the \\
ventilation system \\
and ultimately \\
vessel ventilation \\
system failure.
\end{tabular} & \begin{tabular}{|l|} 
High water level in \\
separator (Vac vessel \\
T03) results in overflow \\
to vessel vent header and \\
into blower. Lose \\
ventilation capability and \\
water in system.
\end{tabular} & 1 & 0 & 3 & $\begin{array}{l}\text { Separator level control (valve and } \\
\text { level switch) }\end{array}$ & Operator rounds & & E0 & 2 \\
\hline PEP-90-13-01 & 13 & $\begin{array}{l}\text { Personnel injury } \\
\text { due to burn. }\end{array}$ & $\begin{array}{l}\text { Material temperature too } \\
\text { high. Sample taken burns } \\
\text { personnel. }\end{array}$ & 1 & 1 & 2 & $\begin{array}{l}\text { Design of the sample vessel } \\
\text { PPE } \\
\text { Operating procedures }\end{array}$ & \begin{tabular}{|l|} 
PPE \\
Operating procedures
\end{tabular} & $\begin{array}{l}\text { There is a smaller } \\
\text { surface area potential } \\
\text { with the sample } \\
\text { container therefore } \\
\text { the consequence is a } \\
1 .\end{array}$ & E0 & SD \\
\hline PEP-90-13-02 & 13 & $\begin{array}{l}\text { Small release of } \\
\text { material to the } \\
\text { facility. }\end{array}$ & $\begin{array}{l}\text { Sample valve } \\
\text { inadvertently left open. } \\
\text { Leak of material to the } \\
\text { facility. }\end{array}$ & 1 & 2 & 3 & $\begin{array}{l}\text { PPE } \\
\text { Operating procedures } \\
\text { Design incorporates a sample } \\
\text { containment area }\end{array}$ & \begin{tabular}{|l|} 
PPE \\
Operating procedures
\end{tabular} & $\begin{array}{l}\text { Limited volume } \\
\text { available for } \\
\text { sampling. } \\
\text { Consequence not } \\
\text { increased for second } \\
\text { person. }\end{array}$ & E0 & SD \\
\hline PEP-90-13-03 & 13 & Operational upset. & $\begin{array}{l}\text { Open up vacuum for } \\
\text { priming. Pull material } \\
\text { from slurry recirculation } \\
\text { system up to vacuum pot. } \\
\text { Overflow into vacuum } \\
\text { system, failing system. } \\
\end{array}$ & & 0 & $\mathrm{n} / \mathrm{a}$ & & $\mathrm{n} / \mathrm{a}$ & & E0 & \\
\hline PEP-90-13-04 & 13 & $\begin{array}{l}\text { Small release of } \\
\text { material to the } \\
\text { facility. }\end{array}$ & $\begin{array}{l}\text { When charging, vacuum } \\
\text { system liquid accumulates } \\
\text { in vacuum pot. Prior to } \\
\text { sampling, pot is dropped } \\
\text { and emptied, spilling } \\
\text { accumulated material } \\
\text { (max } 2 \text { liters). }\end{array}$ & 1 & 2 & 3 & \begin{tabular}{|l|} 
PPE \\
Operating procedures
\end{tabular} & PPE & $\begin{array}{l}\text { Limited volume } \\
\text { available for } \\
\text { sampling. } \\
\text { Consequence not } \\
\text { increased for second } \\
\text { person. }\end{array}$ & E0 & SD \\
\hline
\end{tabular}


Table E.1. Hazard Analysis Worksheets

\begin{tabular}{|c|c|c|c|c|c|c|c|c|c|c|c|}
\hline $\begin{array}{l}\text { Scenario } \\
\text { Number }\end{array}$ & Node & $\begin{array}{l}\text { Hazardous } \\
\text { Condition }\end{array}$ & Cause & L & C & $\begin{array}{l}\text { Risk } \\
\text { Score }\end{array}$ & Potential Safeguard & $\begin{array}{l}\text { Credited } \\
\text { Safeguards }\end{array}$ & $\begin{array}{l}\text { Comments/ } \\
\text { Assumptions }\end{array}$ & E & $\$$ \\
\hline PEP-30-A-01 & A1 & $\begin{array}{l}\text { Personnel injury } \\
\text { due to fall. }\end{array}$ & $\begin{array}{l}\text { Operator falls when } \\
\text { climbing stairs/ladder to } \\
\text { get to upper platform or } \\
\text { when performing } \\
\text { operations on platform. } \\
\end{array}$ & 1 & 4 & 5 & $\begin{array}{l}\text { Design of platform, stairs, ladder } \\
\text { (grating, railings) } \\
\text { Operating procedures } \\
\text { Lighting (normal and facility } \\
\text { emergency) }\end{array}$ & \begin{tabular}{|l|} 
Design of platform, \\
stairs, ladder \\
(grating, railings) \\
Lighting (normal and \\
facility emergency) \\
\end{tabular} & & E0 & $\mathrm{SD}$ \\
\hline PEP-30-A-02 & A1 & $\begin{array}{l}\text { Personnel injury } \\
\text { due to impact. }\end{array}$ & $\begin{array}{l}\text { Operator working on } \\
\text { lower level runs into low } \\
\text { hanging pipe or other } \\
\text { equipment. }\end{array}$ & 1 & 2 & 3 & $\begin{array}{l}\text { PPE (hard hats) } \\
\text { Lighting (normal and facility } \\
\text { emergency) }\end{array}$ & \begin{tabular}{|l|} 
PPE (hard hats) \\
Lighting (normal and \\
facility emergency)
\end{tabular} & & E0 & $\mathrm{SD}$ \\
\hline PEP-30-A-03 & A1 & $\begin{array}{l}\text { Personnel injury } \\
\text { due to burn. }\end{array}$ & $\begin{array}{l}\text { Exposure of Operator to } \\
\text { heated surfaces (greater } \\
\text { than } 212 \text { F) i.e., piping, } \\
\text { tanks, etc. }\end{array}$ & 1 & 3 & 4 & $\begin{array}{l}\text { Insulation on piping and tank sides } \\
\text { PPE (gloves) }\end{array}$ & $\begin{array}{l}\text { Insulation on piping } \\
\text { and tank sides } \\
\text { PPE (gloves) }\end{array}$ & $\begin{array}{l}\text { There are still some } \\
\text { equipment design } \\
\text { issues that need to be } \\
\text { resolved for the } \\
\text { protection and access } \\
\text { to the tank tops. This } \\
\text { relates to sampling or } \\
\text { normal operation. }\end{array}$ & E0 & $\mathrm{SD}$ \\
\hline PEP-30-A-04 & A2 & $\begin{array}{l}\text { Personnel injury } \\
\text { due to impact. }\end{array}$ & \begin{tabular}{|l|} 
Operator drops portable \\
pump (or other tool ) \\
when moving on to upper \\
platform. Falls to lower \\
level.
\end{tabular} & 1 & 4 & 5 & $\begin{array}{l}\text { Design of platform, stairs (grating, } \\
\text { railings, toe boards) } \\
\text { PPE (hard hats) }\end{array}$ & $\begin{array}{l}\text { Design of platform, } \\
\text { stairs (grating, } \\
\text { railings, toe boards) } \\
\text { PPE (hard hats) }\end{array}$ & & E0 & $\mathrm{SD}$ \\
\hline PEP-30-A-05 & $\mathrm{A} 2$ & Personnel injury. & $\begin{array}{l}\text { Operator strains muscle or } \\
\text { damages foot etc. when } \\
\text { lifting/dropping portable } \\
\text { pump or other equipment. }\end{array}$ & 1 & 2 & 3 & $\begin{array}{l}\text { PPE (protective footwear) } \\
\text { Personnel training }\end{array}$ & None identified & & E0 & $\mathrm{SD}$ \\
\hline $\mathrm{n} / \mathrm{a}$ & $\mathrm{A} 3$ & & & & & & & & $\begin{array}{l}\text { Same issues as } \\
\text { identified above in A1 } \\
\text { and A2. }\end{array}$ & & \\
\hline PEP-30-A-06 & A4 & $\begin{array}{l}\text { Personnel injury } \\
\text { due to rotating } \\
\text { equipment. }\end{array}$ & \begin{tabular}{|l|} 
During activities on \\
platform with agitator \\
operating, Operator \\
injured by rotating \\
equipment (at lower level \\
interaction with pumps.) \\
\end{tabular} & 1 & 2 & 3 & $\begin{array}{l}\text { Machine guards on agitators and } \\
\text { pumps } \\
\text { Lockout/Tagout Requirements }\end{array}$ & $\begin{array}{l}\text { Machine guards on } \\
\text { agitators and pumps }\end{array}$ & & E0 & $\mathrm{SD}$ \\
\hline PEP-30-A-07 & A4 & $\begin{array}{l}\text { Personnel injury } \\
\text { due to steam burn. }\end{array}$ & $\begin{array}{l}\text { Steam system temporarily } \\
\text { down (or upon start-up). } \\
\text { When restarting system, } \\
\text { condensate is present in } \\
\text { lines, resulting in water } \\
\text { hammer. System fails, } \\
\text { releasing steam. }\end{array}$ & 1 & 5 & 6 & $\begin{array}{l}\text { Design of steam system } \\
\text { Operating procedures }\end{array}$ & $\begin{array}{l}\text { Operating procedures } \\
\text { Design of steam } \\
\text { system to code }\end{array}$ & & E0 & $\mathrm{SD}$ \\
\hline
\end{tabular}


Table E.1. Hazard Analysis Worksheets

\begin{tabular}{|c|c|c|c|c|c|c|c|c|c|c|c|}
\hline $\begin{array}{l}\text { Scenario } \\
\text { Number }\end{array}$ & Node & $\begin{array}{c}\text { Hazardous } \\
\text { Condition }\end{array}$ & Cause & $\mathbf{L}$ & C & $\begin{array}{l}\text { Risk } \\
\text { Score }\end{array}$ & Potential Safeguard & $\begin{array}{c}\text { Credited } \\
\text { Safeguards }\end{array}$ & $\begin{array}{c}\text { Comments/ } \\
\text { Assumptions }\end{array}$ & $\mathbf{E}$ & $\$$ \\
\hline PEP-30-A-08 & A5 & $\begin{array}{l}\text { Personnel heat } \\
\text { stress. }\end{array}$ & $\begin{array}{l}\text { High temperatures in } \\
\text { building environment } \\
\text { (high ambient } \\
\text { temperatures, etc.). }\end{array}$ & 1 & 2 & 3 & $\begin{array}{l}\text { Building HVAC with temperature } \\
\text { monitoring } \\
\text { Administrative controls for stop work } \\
\text { JHA and work plan (PPE etc) }\end{array}$ & \begin{tabular}{|l|} 
Building HVAC with \\
temperature \\
monitoring \\
Administrative \\
controls for stop \\
work
\end{tabular} & & E0 & $\mathrm{SD}$ \\
\hline PEP-30-A-09 & A5 & $\begin{array}{l}\text { Personnel injury } \\
\text { due to tripping, } \\
\text { falling. }\end{array}$ & \begin{tabular}{|l|} 
Loss of facility power \\
when facility manned. \\
Hazard for tripping, \\
falling etc. due to \\
darkness. Potential to fall \\
from elevated structures.
\end{tabular} & 1 & 4 & 5 & $\begin{array}{l}\text { Design of platform, stairs, ladder } \\
\text { (railings) } \\
\text { Lighting (facility emergency) }\end{array}$ & $\begin{array}{l}\text { Lighting (facility } \\
\text { emergency) }\end{array}$ & & E0 & $\mathrm{SD}$ \\
\hline PEP-30-A-10 & A5 & $\begin{array}{l}\text { Personnel injury } \\
\text { due to high noise } \\
\text { levels. }\end{array}$ & $\begin{array}{l}\text { High noise levels from } \\
\text { equipment operation. }\end{array}$ & 1 & 2 & 3 & $\begin{array}{l}\text { All high-noise equipment is located } \\
\text { outside the facility }\end{array}$ & $\begin{array}{l}\text { PPE (hearing } \\
\text { protection) - if } \\
\text { needed }\end{array}$ & $\begin{array}{l}\text { Sound level survey } \\
\text { needs to be performed } \\
\text { upon startup to } \\
\text { determine the noise } \\
\text { level in the facility. }\end{array}$ & E0 & $\mathrm{SD}$ \\
\hline PEP-30-B-01 & B1 & $\begin{array}{l}\text { Facility fire. } \\
\text { Personnel injury } \\
\text { due to vehicle } \\
\text { impact and/or fire. } \\
\text { Damage to facility } \\
\text { due to impact and } \\
\text { fire. }\end{array}$ & \begin{tabular}{|l|} 
Vehicle collision impacts \\
facility and ruptures fuel \\
tank, resulting in fire \\
adjacent to facility. \\
Interact with chemicals \\
being received outside \\
(maximum of 2 totes.)
\end{tabular} & -1 & 4 & 3 & \begin{tabular}{|l} 
Fire alarm \\
Sprinkler system \\
Emergency preparedness \\
Speed limits (PNNL)
\end{tabular} & $\begin{array}{l}\text { Fire alarm } \\
\text { Sprinkler system }\end{array}$ & $\begin{array}{l}\text { Chemicals will be } \\
\text { received in the liquid } \\
\text { form (no dry mixing } \\
\text { required). } \\
\text { Maximum of } 2 \text { totes } \\
\text { will be received at } \\
\text { one time. }\end{array}$ & E0 & $\mathrm{SD}$ \\
\hline PEP-30-B-02 & B1 & $\begin{array}{l}\text { Personnel injury } \\
\text { due to impact. } \\
\text { Damage to facility } \\
\text { structure. }\end{array}$ & $\begin{array}{l}\text { Vehicle collision impacts } \\
\text { facility and injures facility } \\
\text { personnel. }\end{array}$ & 0 & 4 & 4 & \begin{tabular}{|l|} 
Speed limits (PNNL) \\
Emergency preparedness
\end{tabular} & Speed limits (PNNL) & & E0 & SD \\
\hline PEP-30-B-03 & B1 & $\begin{array}{l}\text { Personnel injury } \\
\text { due to impact. } \\
\text { Damage to facility } \\
\text { structure. }\end{array}$ & $\begin{array}{l}\text { Drop/swing crane load } \\
\text { during chemical/ } \\
\text { equipment off-loading or } \\
\text { moving (replacement). } \\
\text { Impact facility and/or } \\
\text { personnel. }\end{array}$ & 0 & 4 & 4 & $\begin{array}{l}\text { Hoisting, Rigging, Forklifts, and } \\
\text { Aerial Lifts Requirements } \\
\text { Critical lift plan (for initial installation } \\
\text { of equipment) } \\
\text { Operating procedures } \\
\text { Personnel training/qualification }\end{array}$ & $\begin{array}{l}\text { Hoisting, Rigging, } \\
\text { Forklifts, and Aerial } \\
\text { Lifts Requirements }\end{array}$ & & E0 & $\mathrm{SD}$ \\
\hline PEP-30-B-04 & B1 & $\begin{array}{l}\text { Personnel injury } \\
\text { due to exposure to } \\
\text { chemicals. } \\
\text { Release of } \\
\text { chemicals to the } \\
\text { environment. }\end{array}$ & $\begin{array}{l}\text { Drop forklift load, or hit } \\
\text { load with forklift, during } \\
\text { chemical off- } \\
\text { loading/moving. Failure } \\
\text { of tote/drum releasing } \\
\text { chemicals. }\end{array}$ & 0 & 4 & 4 & $\begin{array}{l}\text { Hoisting, Rigging, Forklifts, and } \\
\text { Aerial Lifts Requirements } \\
\text { Operating procedures } \\
\text { Personnel training/qualification } \\
\text { Design of containers (DOT) } \\
\text { Spill response } \\
\text { Safety shower/eyewash }\end{array}$ & $\begin{array}{l}\text { Hoisting, Rigging, } \\
\text { Forklifts, and Aerial } \\
\text { Lifts Requirements }\end{array}$ & & E2 & SD \\
\hline
\end{tabular}


Table E.1. Hazard Analysis Worksheets

\begin{tabular}{|c|c|c|c|c|c|c|c|c|c|c|c|}
\hline $\begin{array}{l}\text { Scenario } \\
\text { Number }\end{array}$ & Node & $\begin{array}{c}\text { Hazardous } \\
\text { Condition }\end{array}$ & Cause & $\mathbf{L}$ & C & $\begin{array}{l}\text { Risk } \\
\text { Score }\end{array}$ & Potential Safeguard & $\begin{array}{c}\text { Credited } \\
\text { Safeguards }\end{array}$ & $\begin{array}{c}\text { Comments/ } \\
\text { Assumptions }\end{array}$ & $\mathbf{E}$ & $\$$ \\
\hline PEP-30-B-05 & B1 & $\begin{array}{l}\text { Personnel injury } \\
\text { due to impact. } \\
\text { Damage to facility } \\
\text { structure. }\end{array}$ & $\begin{array}{l}\text { Drop forklift load onto } \\
\text { operator or hit with } \\
\text { forklift when off-loading } \\
\text { or moving (replacement) } \\
\text { of chemicals/equipment. } \\
\text { Potential for impact to } \\
\text { facility structure. }\end{array}$ & 0 & 4 & 4 & $\begin{array}{l}\text { Hoisting, Rigging, Forklifts, and } \\
\text { Aerial Lifts Requirements } \\
\text { Operating procedures } \\
\text { Personnel training/qualification }\end{array}$ & $\begin{array}{l}\text { Hoisting, Rigging, } \\
\text { Forklifts, and Aerial } \\
\text { Lifts Requirements }\end{array}$ & & E0 & $\mathrm{SD}$ \\
\hline PEP-30-B-06 & B2 & $\begin{array}{l}\text { Personnel injury } \\
\text { due to exposure to } \\
\text { material. Large } \\
\text { release of } \\
\text { permeate or } \\
\text { simulant to } \\
\text { ground. }\end{array}$ & $\begin{array}{l}\text { Failure to connect the flex } \\
\text { hose correctly to the } \\
\text { tanker truck (or failure of } \\
\text { system) during material } \\
\text { transfer (simulant in or } \\
\text { permeate out). Valves } \\
\text { opened and truck contents } \\
\text { spilled to ground. }\end{array}$ & 0 & 4 & 4 & $\begin{array}{l}\text { Operating procedures } \\
\text { Procurement specifications (fitting } \\
\text { type) } \\
\text { PPE } \\
\text { Safety showers/eye wash }\end{array}$ & \begin{tabular}{|l|} 
Operating procedures \\
PPE \\
Safety showers/eye \\
wash
\end{tabular} & $\begin{array}{l}\text { The consequence is } \\
\text { rated on the } \\
\text { possibility of multiple } \\
\text { injuries (driver and } \\
\text { operator.) }\end{array}$ & E2 & $\mathrm{SD}$ \\
\hline PEP-30-B-07 & $\mathrm{B} 2$ & $\begin{array}{l}\text { Personnel injury } \\
\text { due to splash of } \\
\text { material. Small } \\
\text { spill of permeate } \\
\text { or simulant to } \\
\text { ground. }\end{array}$ & $\begin{array}{l}\text { Failure to connect the flex } \\
\text { hose correctly to the } \\
\text { tanker truck (or failure of } \\
\text { system) during material } \\
\text { transfer (simulant in or } \\
\text { permeate out). Spill of } \\
\text { material outside. }\end{array}$ & 1 & 1 & 2 & $\begin{array}{l}\text { Operating procedures } \\
\text { Procurement specifications (fitting } \\
\text { type) } \\
\text { PPE } \\
\text { Safety showers/eye wash }\end{array}$ & $\begin{array}{l}\text { Operating procedures } \\
\text { PPE } \\
\text { Safety showers/eye } \\
\text { wash }\end{array}$ & $\begin{array}{l}\text { Small spill to the } \\
\text { driveway is not } \\
\text { reportable. }\end{array}$ & E0 & $\mathrm{SD}$ \\
\hline PEP-30-B-08 & B3 & $\begin{array}{l}\text { Personnel injury } \\
\text { due to } \\
\text { explosion/fire. } \\
\text { Damage to facility } \\
\text { from fire. }\end{array}$ & $\begin{array}{l}\text { Forklift propane tank fire } \\
\text { inside facility. }\end{array}$ & -2 & 4 & 2 & $\begin{array}{l}\text { Design of vendor supplied propane } \\
\text { tanks } \\
\text { Personnel training/qualification } \\
\text { Fire alarm } \\
\text { Sprinkler system } \\
\text { Emergency preparedness }\end{array}$ & $\begin{array}{l}\text { Fire alarm } \\
\text { Sprinkler system }\end{array}$ & & E0 & $\mathrm{SD}$ \\
\hline $\mathrm{n} / \mathrm{a}$ & B4 & & & & & & & & $\begin{array}{l}\text { Same issues as } \\
\text { identified above in } \\
\text { B1, B2 and B3. }\end{array}$ & & \\
\hline $\mathrm{n} / \mathrm{a}$ & B5 & & & & & & & & $\begin{array}{l}\text { Same issues as } \\
\text { identified above in } \\
\mathrm{B} 1, \mathrm{~B} 2 \text { and B3. } \\
\end{array}$ & & \\
\hline $\mathrm{n} / \mathrm{a}$ & B6 & & & & & & & & $\begin{array}{l}\text { Same issues as } \\
\text { identified above in } \\
\mathrm{B} 1, \mathrm{~B} 2 \text { and B3. }\end{array}$ & & \\
\hline
\end{tabular}


Table E.1. Hazard Analysis Worksheets

\begin{tabular}{|c|c|c|c|c|c|c|c|c|c|c|c|}
\hline $\begin{array}{l}\text { Scenario } \\
\text { Number }\end{array}$ & Node & $\begin{array}{l}\text { Hazardous } \\
\text { Condition }\end{array}$ & Cause & $\mathbf{L}$ & C & $\begin{array}{l}\text { Risk } \\
\text { Score }\end{array}$ & Potential Safeguard & $\begin{array}{l}\text { Credited } \\
\text { Safeguards }\end{array}$ & $\begin{array}{l}\text { Comments/ } \\
\text { Assumptions }\end{array}$ & $\mathbf{E}$ & $\$$ \\
\hline PEP-30-C-01 & $\mathrm{C} 1$ & $\begin{array}{l}\text { Personnel injury } \\
\text { due to exposure to } \\
\text { chemicals. Spill } \\
\text { of material inside } \\
\text { facility. }\end{array}$ & $\begin{array}{l}\text { During chemical receipt, } \\
\text { add wrong chemical into } \\
\text { wrong receipt tank (acid } \\
\text { into caustic or reverse). } \\
\text { Chemical reaction causes } \\
\text { high heat, tank failure, } \\
\text { reaction aerosol release. }\end{array}$ & 1 & 4 & 5 & $\begin{array}{l}\text { Facility secondary containment } \\
\text { Incompatible fitting design between } \\
\text { chemical types } \\
\text { Operating procedures } \\
\text { Chemical pipe labeling/color coding } \\
\text { Receipt tank pressure relief } \\
\text { Receipt tank ventilation system }\end{array}$ & \begin{tabular}{|l|} 
Incompatible fitting \\
design between \\
chemical types \\
Operating procedures
\end{tabular} & & E0 & $\mathrm{SD}$ \\
\hline PEP-30-C-02 & $\mathrm{C} 1$ & $\begin{array}{l}\text { Personnel injury } \\
\text { due to exposure to } \\
\text { chemicals. Spill } \\
\text { of material inside } \\
\text { facility. }\end{array}$ & $\begin{array}{l}\text { Misvalving in chemical } \\
\text { addition system causes } \\
\text { misroute to open path. } \\
\text { Spill to the facility. }\end{array}$ & 1 & 3 & 4 & $\begin{array}{l}\text { PPE } \\
\text { Operating procedures (valve lineup) } \\
\text { Facility secondary containment }\end{array}$ & $\begin{array}{l}\text { Operating procedures } \\
\text { PPE }\end{array}$ & & E0 & $\mathrm{SD}$ \\
\hline PEP-30-C-04 & $\mathrm{C} 1$ & $\begin{array}{l}\text { Personnel injury } \\
\text { due to electrical } \\
\text { shock. }\end{array}$ & $\begin{array}{l}\text { Personnel in contact with } \\
\text { heat trace system results } \\
\text { in electrical shock. }\end{array}$ & 1 & 4 & 5 & $\begin{array}{l}\text { UL listing } \\
\text { National Electric Code compliance }\end{array}$ & UL listed heat trace & & E0 & $\mathrm{SD}$ \\
\hline $\mathrm{n} / \mathrm{a}$ & $\mathrm{C} 2$ & & & & & & & & $\begin{array}{l}\text { Same issues as } \\
\text { identified above in } \\
\text { C1. }\end{array}$ & & \\
\hline PEP-30-C-05 & $\mathrm{C} 3$ & $\begin{array}{l}\text { Personnel injury } \\
\text { due to exposure to } \\
\text { chemicals. Small } \\
\text { spill of material } \\
\text { inside facility. }\end{array}$ & $\begin{array}{l}\text { Overfill sample container, } \\
\text { open valve with container } \\
\text { mis-positioned or missing, } \\
\text { or drop sample container. } \\
\text { Splash operator and spill } \\
\text { to facility. }\end{array}$ & 1 & 2 & 3 & $\begin{array}{l}\text { Control volume and flow rate of } \\
\text { sample location } \\
\text { Operating procedures } \\
\text { PPE (gloves, safety glasses) } \\
\text { Facility secondary containment }\end{array}$ & \begin{tabular}{|l|} 
PPE \\
Operating procedures
\end{tabular} & $\begin{array}{l}\text { All samples ports for } \\
\text { the chemical addition } \\
\text { system are on low } \\
\text { pressure points of } \\
\text { system. } \\
\text { Limited volume } \\
\text { available for } \\
\text { sampling. } \\
\text { Consequence not } \\
\text { increased for second } \\
\text { person. }\end{array}$ & E0 & $\mathrm{SD}$ \\
\hline PEP-30-C-06 & $\mathrm{C} 3$ & $\begin{array}{l}\text { Personnel injury } \\
\text { due to exposure to } \\
\text { chemicals. Small } \\
\text { spill of material } \\
\text { inside facility. }\end{array}$ & $\begin{array}{l}\text { Impact or damage to } \\
\text { sample carrier during } \\
\text { transport through the } \\
\text { facility. Spill of } \\
\text { approximately } \\
10 \text { samples. }\end{array}$ & 1 & 2 & 3 & $\begin{array}{l}\text { Unbreakable, closed sample } \\
\text { containers } \\
\text { Facility secondary containment } \\
\text { PPE (gloves, safety glasses) }\end{array}$ & \begin{tabular}{|l|} 
Operating procedures \\
PPE \\
Design of sample \\
container
\end{tabular} & & E0 & $\mathrm{SD}$ \\
\hline PEP-30-D-01 & D1 & $\begin{array}{l}\text { Personnel injury } \\
\text { due to burn. }\end{array}$ & $\begin{array}{l}\text { Contact with hot surfaces } \\
\text { (piping) during sample } \\
\text { acquisition. }\end{array}$ & 1 & 1 & 2 & $\begin{array}{l}\text { Insulation on piping } \\
\text { PPE (gloves) }\end{array}$ & $\begin{array}{l}\text { Insulation on piping } \\
\text { PPE (gloves) }\end{array}$ & $\begin{array}{l}\text { The consequences are } \\
\text { limited due to the } \\
\text { smaller surface area. }\end{array}$ & E0 & SD \\
\hline PEP-30-D-02 & D1 & $\begin{array}{l}\text { Personnel injury } \\
\text { due to exposure to } \\
\text { process material. } \\
\text { Release of }\end{array}$ & $\begin{array}{l}\text { Open a sample port when } \\
\text { the line is pressurized; } \\
\text { results in pressurized } \\
\text { release of process }\end{array}$ & 1 & 2 & 3 & $\begin{array}{l}\text { Operating procedures } \\
\text { PPE (gloves, safety glasses) } \\
\text { Design location of sample ports } \\
\text { Pressure indication on sample line }\end{array}$ & $\begin{array}{l}\text { Operating procedures } \\
\text { PPE (gloves, safety } \\
\text { glasses) }\end{array}$ & $\begin{array}{l}\text { Design places the } \\
\text { sample ports on the } \\
\text { low pressure points of } \\
\text { the system. }\end{array}$ & E0 & SD \\
\hline
\end{tabular}


Table E.1. Hazard Analysis Worksheets

\begin{tabular}{|c|c|c|c|c|c|c|c|c|c|c|c|}
\hline $\begin{array}{l}\text { Scenario } \\
\text { Number }\end{array}$ & Node & $\begin{array}{c}\text { Hazardous } \\
\text { Condition }\end{array}$ & Cause & $\mathbf{L}$ & C & $\begin{array}{c}\text { Risk } \\
\text { Score } \\
\end{array}$ & Potential Safeguard & $\begin{array}{c}\text { Credited } \\
\text { Safeguards } \\
\end{array}$ & $\begin{array}{c}\text { Comments/ } \\
\text { Assumptions }\end{array}$ & E & $\$$ \\
\hline & & $\begin{array}{l}\text { material to the } \\
\text { facility. }\end{array}$ & materials. & & & & & & & & \\
\hline PEP-30-D-03 & D1 & $\begin{array}{l}\text { Personnel injury } \\
\text { due to exposure to } \\
\text { high-temperature } \\
\text { process material. }\end{array}$ & $\begin{array}{l}\text { Pull a sample of high } \\
\text { temperature material. } \\
\text { Sample container breaks } \\
\text { from the heat. }\end{array}$ & 1 & 1 & 2 & $\begin{array}{l}\text { Operating procedures } \\
\text { PPE (gloves, safety glasses) }\end{array}$ & $\begin{array}{l}\text { Design of sample } \\
\text { container } \\
\text { PPE }\end{array}$ & $\begin{array}{l}\text { All samples ports for } \\
\text { the chemical addition } \\
\text { system are on low } \\
\text { pressure points of } \\
\text { system. } \\
\text { Limited volume } \\
\text { available for } \\
\text { sampling. } \\
\text { Consequence not } \\
\text { increased for second } \\
\text { person. }\end{array}$ & E0 & SD \\
\hline PEP-30-D-04 & D1 & $\begin{array}{l}\text { Personnel injury } \\
\text { due to burn. }\end{array}$ & $\begin{array}{l}\text { Pull a sample of high- } \\
\text { temperature material. } \\
\text { Operator is burned by hot } \\
\text { temperature surface of } \\
\text { sample container. }\end{array}$ & 1 & 1 & 2 & $\begin{array}{l}\text { Operating procedures } \\
\text { PPE (gloves, safety glasses) }\end{array}$ & $\begin{array}{l}\text { PPE (gloves, safety } \\
\text { glasses) }\end{array}$ & & E0 & SD \\
\hline PEP-FN-D-01 & D2 & \begin{tabular}{|l|} 
Personnel \\
exposure to hot \\
chemicals. Small \\
release to facility.
\end{tabular} & $\begin{array}{l}\text { Spill of sample when } \\
\text { transferring within } \\
\text { facility. }\end{array}$ & 1 & 1 & 2 & $\begin{array}{l}\text { Operating procedures } \\
\text { PPE } \\
\text { Design of sample vessel }\end{array}$ & $\begin{array}{l}\text { Operating procedures } \\
\text { PPE } \\
\text { Design of sample } \\
\text { container }\end{array}$ & & E0 & $\mathrm{SD}$ \\
\hline PEP-FN-D-02 & $\mathrm{D} 2$ & $\begin{array}{l}\text { Personnel } \\
\text { exposure to hot } \\
\text { chemicals. Small } \\
\text { release to facility. }\end{array}$ & $\begin{array}{l}\text { Mishandling of sample } \\
\text { during analysis activities } \\
\text { exposes personnel to } \\
\text { chemicals. }\end{array}$ & 1 & 1 & 2 & $\begin{array}{l}\text { Operating procedures } \\
\text { PPE }\end{array}$ & $\begin{array}{l}\text { Operating procedures } \\
\text { PPE }\end{array}$ & & E0 & SD \\
\hline PEP-FN-D-03 & D3 & $\begin{array}{l}\text { Small release of } \\
\text { material outside of } \\
\text { facility. }\end{array}$ & $\begin{array}{l}\text { Spill of sample tray when } \\
\text { transferring to archive } \\
\text { area. }\end{array}$ & 1 & 1 & 2 & $\begin{array}{l}\text { Operating procedures } \\
\text { PPE } \\
\text { Design of sample container }\end{array}$ & $\begin{array}{l}\text { Operating procedures } \\
\text { PPE } \\
\text { Design of sample } \\
\text { container }\end{array}$ & $\begin{array}{l}\text { A tray could hold a } \\
\text { dozen sample bottles }\end{array}$ & E1 & SD \\
\hline PEP-FN-D-04 & D3 & $\begin{array}{l}\text { Release of } \\
\text { material outside } \\
\text { facility. }\end{array}$ & $\begin{array}{l}\text { Vehicle collision with } \\
\text { sample archive area. }\end{array}$ & -1 & 1 & 0 & \begin{tabular}{|l|} 
PNNL speed limits \\
Emergency preparedness \\
Personnel training and qualifications \\
Design of sample cabinet (Conex) \\
Secondary confinement on sample \\
archive cabinet.
\end{tabular} & $\begin{array}{l}\text { Speed limits (PNNL) } \\
\text { Design of sample } \\
\text { cabinet (Conex) } \\
\text { Secondary } \\
\text { confinement on } \\
\text { sample archive } \\
\text { cabinet. }\end{array}$ & & E1 & SD \\
\hline
\end{tabular}


Table E.1. Hazard Analysis Worksheets

\begin{tabular}{|c|c|c|c|c|c|c|c|c|c|c|c|}
\hline $\begin{array}{l}\text { Scenario } \\
\text { Number }\end{array}$ & Node & $\begin{array}{l}\text { Hazardous } \\
\text { Condition }\end{array}$ & Cause & $\mathbf{L}$ & C & $\begin{array}{l}\text { Risk } \\
\text { Score }\end{array}$ & Potential Safeguard & $\begin{array}{c}\text { Credited } \\
\text { Safeguards }\end{array}$ & $\begin{array}{l}\text { Comments/ } \\
\text { Assumptions }\end{array}$ & $\mathbf{E}$ & $\$$ \\
\hline PEP-FN-D-05 & D3 & \begin{tabular}{|l} 
Release of \\
material outside \\
facility.
\end{tabular} & $\begin{array}{l}\text { Extreme environmental } \\
\text { conditions. Sample } \\
\text { containers in cabinet fail. }\end{array}$ & 0 & 1 & 1 & $\begin{array}{l}\text { Design of sample container } \\
\text { Secondary confinement on sample } \\
\text { archive cabinet. }\end{array}$ & $\begin{array}{l}\text { Design of sample } \\
\text { container } \\
\text { Secondary } \\
\text { confinement on } \\
\text { sample archive } \\
\text { cabinet. }\end{array}$ & & E1 & SD \\
\hline PEP-30-E-01 & E2 & $\begin{array}{l}\text { Personnel injury } \\
\text { due to exposure to } \\
\text { process material. } \\
\text { Release of } \\
\text { material to the } \\
\text { facility. }\end{array}$ & $\begin{array}{l}\text { Recovery actions to a } \\
\text { plugged line in system. } \\
\text { Disconnect flanges, } \\
\text { removing valve bodies, } \\
\text { etc. Spill material to the } \\
\text { facility. }\end{array}$ & 1 & 3 & 4 & JHA and work plan (PPE etc) & JHA and work plan & & E0 & SD \\
\hline PEP-30-E-02 & E2 & $\begin{array}{l}\text { Personnel injury } \\
\text { due to exposure to } \\
\text { pressurized } \\
\text { process material. } \\
\text { Release of } \\
\text { material to the } \\
\text { facility. }\end{array}$ & $\begin{array}{l}\text { Recovery actions to a } \\
\text { plugged line in system. } \\
\text { Disconnect flanges, } \\
\text { removing valve bodies, } \\
\text { etc. When open system, } \\
\text { back pressure releases } \\
\text { material to the facility. }\end{array}$ & 1 & 3 & 4 & \begin{tabular}{|l} 
JHA and work plan (PPE etc) \\
Facility secondary containment
\end{tabular} & JHA and work plan & & E0 & SD \\
\hline PEP-30-E-03 & E2 & $\begin{array}{l}\text { Personnel injury } \\
\text { due to impact. }\end{array}$ & $\begin{array}{l}\text { Recovery actions to a } \\
\text { plugged line in system. } \\
\text { Disconnect flanges, } \\
\text { removing valve bodies, } \\
\text { etc. Drop equipment } \\
\text { when moving (manually } \\
\text { or with crane). }\end{array}$ & 1 & 4 & 5 & $\begin{array}{l}\text { JHA and work plan (PPE etc) } \\
\text { Design of platform, stairs (grating, } \\
\text { railings, toe boards) } \\
\text { Hard hats } \\
\text { Protective footwear } \\
\text { Personnel training and qualifications } \\
\text { Hoisting, Rigging, Forklifts, and } \\
\text { Aerial Lifts Requirements }\end{array}$ & JHA and work plan & & E0 & SD \\
\hline PEP-30-E-04 & E2 & $\begin{array}{l}\text { Personnel injury } \\
\text { due to steam burn. }\end{array}$ & $\begin{array}{l}\text { Recovery/repair actions } \\
\text { (plugged process line, } \\
\text { replacing ultrafilter, etc.). } \\
\text { Failure to de-energize the } \\
\text { steam system. When } \\
\text { system opened, release } \\
\text { steam. }\end{array}$ & 1 & 4 & 5 & $\begin{array}{l}\text { Lockout/Tagout Requirements } \\
\text { JHA and work plan (PPE etc) } \\
\text { Double isolation valves }\end{array}$ & JHA and work plan & & E0 & SD \\
\hline PEP-30-E-05 & E2 & $\begin{array}{l}\text { Personnel injury } \\
\text { due to electrical } \\
\text { shock. }\end{array}$ & $\begin{array}{l}\text { Recovery/repair actions } \\
\text { (plugged line in system, } \\
\text { replacing ultrafilter, etc.). } \\
\text { Failure to de-energize the } \\
\text { electrical system (heat } \\
\text { trace). Operator interacts } \\
\text { with system. }\end{array}$ & 1 & 4 & 5 & $\begin{array}{l}\text { UL listing } \\
\text { National Electric Code compliance } \\
\text { Lockout/Tagout Requirements } \\
\text { JHA and work plan (PPE etc) }\end{array}$ & JHA and work plan & & E0 & SD \\
\hline
\end{tabular}


Table E.1. Hazard Analysis Worksheets

\begin{tabular}{|c|c|c|c|c|c|c|c|c|c|c|c|}
\hline $\begin{array}{l}\text { Scenario } \\
\text { Number }\end{array}$ & Node & $\begin{array}{l}\text { Hazardous } \\
\text { Condition }\end{array}$ & Cause & $\mathbf{L}$ & C & $\begin{array}{l}\text { Risk } \\
\text { Score }\end{array}$ & Potential Safeguard & $\begin{array}{l}\text { Credited } \\
\text { Safeguards }\end{array}$ & $\begin{array}{l}\text { Comments/ } \\
\text { Assumptions }\end{array}$ & $\mathbf{E}$ & $\$$ \\
\hline PEP-30-E-06 & E2 & $\begin{array}{l}\text { Personnel injury } \\
\text { due to impact with } \\
\text { missile. }\end{array}$ & $\begin{array}{l}\text { Recovery/repair actions } \\
\text { (plugged line in system, } \\
\text { replacing ultrafilter, etc.). } \\
\text { Failure to de-energize the } \\
\text { compressed air. Open } \\
\text { system resulting in high } \\
\text { pressure release of air. } \\
\text { Air stream (300 psi) lifts } \\
\text { debris. }\end{array}$ & 1 & 4 & 5 & $\begin{array}{l}\text { Lockout/Tagout Requirements } \\
\text { Isolation valves on compressed air } \\
\text { system JHA and work plan (PPE etc) }\end{array}$ & JHA and work plan & & E0 & SD \\
\hline $\mathrm{n} / \mathrm{a}$ & E2 & & & & & & & & $\begin{array}{l}\text { The spill response } \\
\text { plan will address the } \\
\text { recovery actions from } \\
\text { a major spill. The } \\
\text { plan should be } \\
\text { reviewed, when } \\
\text { developed, for its } \\
\text { consistency with the } \\
\text { developed hazards. }\end{array}$ & & \\
\hline PEP-30-E-07 & E2 & $\begin{array}{l}\text { Personnel injury } \\
\text { due to steam burn. }\end{array}$ & $\begin{array}{l}\text { Recovery actions to a } \\
\text { contaminated ventilation } \\
\text { system (carry over of } \\
\text { material). Failure to de- } \\
\text { energize the steam } \\
\text { system. When system } \\
\text { opened, release steam. }\end{array}$ & 1 & 4 & 5 & $\begin{array}{l}\text { Lockout/Tagout Requirements } \\
\text { JHA and work plan (PPE etc) } \\
\text { Double isolation valves }\end{array}$ & JHA and work plan & & E0 & SD \\
\hline PEP-30-E-08 & E2 & $\begin{array}{l}\text { Personnel injury } \\
\text { due to electrical } \\
\text { shock. }\end{array}$ & $\begin{array}{l}\text { Recovery actions to a } \\
\text { contaminated ventilation } \\
\text { system (carry over of } \\
\text { material). Failure to de- } \\
\text { energize the electrical } \\
\text { system. Operator } \\
\text { interacts with electrical } \\
\text { system. }\end{array}$ & 1 & 4 & 5 & $\begin{array}{l}\text { UL listing } \\
\text { National Electric Code compliance } \\
\text { Lockout/Tagout Requirements } \\
\text { JHA and work plan (PPE etc) }\end{array}$ & JHA and work plan & & E0 & SD \\
\hline PEP-30-E-09 & E2 & $\begin{array}{l}\text { Personnel injury } \\
\text { due to exposure to } \\
\text { carry-over } \\
\text { material. Release } \\
\text { of material to the } \\
\text { facility. }\end{array}$ & $\begin{array}{l}\text { Recovery actions to a } \\
\text { contaminated ventilation } \\
\text { system (carry-over of } \\
\text { material). Spill carryover } \\
\text { material when breaking } \\
\text { open the system. } \\
\end{array}$ & 1 & 3 & 4 & \begin{tabular}{|l|} 
JHA and work plan (PPE etc) \\
Facility secondary containment
\end{tabular} & JHA and work plan & & E0 & SD \\
\hline $\mathrm{n} / \mathrm{a}$ & E3 & & & & & & & & & & \\
\hline PEP-30-E-10 & E4 & $\begin{array}{l}\text { Personnel injury } \\
\text { due to exposure to }\end{array}$ & $\begin{array}{l}\text { Failure to drain system } \\
\text { prior to removing a failed }\end{array}$ & 1 & 3 & 4 & $\begin{array}{l}\text { Operating/maintenance procedures } \\
\text { JHA and work plan }\end{array}$ & JHA and work plan & $\begin{array}{l}\text { If we suspect the tube } \\
\text { is failed the system }\end{array}$ & E0 & SD \\
\hline
\end{tabular}


Table E.1. Hazard Analysis Worksheets

\begin{tabular}{|c|c|c|c|c|c|c|c|c|c|c|c|}
\hline $\begin{array}{l}\text { Scenario } \\
\text { Number }\end{array}$ & Node & $\begin{array}{l}\text { Hazardous } \\
\text { Condition }\end{array}$ & Cause & $\mathbf{L}$ & C & $\begin{array}{l}\text { Risk } \\
\text { Score }\end{array}$ & Potential Safeguard & $\begin{array}{l}\text { Credited } \\
\text { Safeguards }\end{array}$ & $\begin{array}{l}\text { Comments/ } \\
\text { Assumptions }\end{array}$ & $\mathbf{E}$ & $\$$ \\
\hline & & $\begin{array}{l}\text { process material. } \\
\text { Release of } \\
\text { material to the } \\
\text { facility. }\end{array}$ & \begin{tabular}{|l} 
ultrafilter or other failed \\
equipment. Process \\
material left in lines spills \\
to facility floor.
\end{tabular} & & & & $\begin{array}{l}\text { Lockout/Tagout Requirements } \\
\text { PPE }\end{array}$ & & $\begin{array}{l}\text { will be flushed with } \\
\text { inhibited water before } \\
\text { the system is opened. } \\
\text { Volume is } \\
\text { approximately } 50-60 \\
\text { gal per ultrafilter. }\end{array}$ & & \\
\hline PEP-30-E-11 & E4 & $\begin{array}{l}\text { Personnel injury } \\
\text { due to impact. }\end{array}$ & $\begin{array}{l}\text { Load drop from crane } \\
\text { when lifting failed } \\
\text { filter/equipment or } \\
\text { loading replacement filter } \\
\text { into system. }\end{array}$ & 0 & 4 & 4 & $\begin{array}{l}\text { Hoisting, Rigging, Forklifts, and } \\
\text { Aerial Lifts Requirements } \\
\text { Personnel training and qualifications }\end{array}$ & JHA and work plan & & E0 & $\mathrm{SD}$ \\
\hline PEP-30-E-12 & E4 & $\begin{array}{l}\text { Personnel injury } \\
\text { due to impact. }\end{array}$ & $\begin{array}{l}\text { Swing crane load when } \\
\text { lifting failed filter or } \\
\text { loading replacement filter } \\
\text { into system. Impact with } \\
\text { personnel. }\end{array}$ & 0 & 4 & 4 & $\begin{array}{l}\text { Hoisting, Rigging, Forklifts, and } \\
\text { Aerial Lifts Requirements } \\
\text { Personnel training and qualifications }\end{array}$ & JHA and work plan & & E0 & $\mathrm{SD}$ \\
\hline PEP-30-E-13 & E4 & $\begin{array}{l}\text { Damage to nearby } \\
\text { equipment. }\end{array}$ & $\begin{array}{l}\text { Drop or swing crane load } \\
\text { when lifting failed } \\
\text { filter/equipment or } \\
\text { loading replacement filter } \\
\text { into system. Impact with } \\
\text { nearby equipment (steam } \\
\text { line, HVAC, heat } \\
\text { exchanger, tank, etc.) }\end{array}$ & 0 & 0 & 2 & $\begin{array}{l}\text { Hoisting, Rigging, Forklifts, and } \\
\text { Aerial Lifts Requirements } \\
\text { Personnel training and qualifications }\end{array}$ & JHA and work plan & & E0 & 2 \\
\hline PEP-30-E-14 & E4 & $\begin{array}{l}\text { Personnel injury } \\
\text { due to tool } \\
\text { operation. }\end{array}$ & $\begin{array}{l}\text { Operator error using } \\
\text { power tools causes } \\
\text { personnel injury. }\end{array}$ & 1 & 2 & 3 & $\begin{array}{l}\text { Maintenance procedures } \\
\text { Personnel training and qualifications } \\
\text { (skill/craft) }\end{array}$ & JHA and work plan & & E0 & SD \\
\hline PEP-30-F-01 & F1 & $\begin{array}{l}\text { Personnel injury } \\
\text { due to exposure to } \\
\text { process waste. }\end{array}$ & $\begin{array}{l}\text { Overfill drum/tote with } \\
\text { process waste. Spill } \\
\text { material inside facility. }\end{array}$ & 1 & 3 & 4 & $\begin{array}{l}\text { Facility secondary containment } \\
\text { Operating procedures }\end{array}$ & $\begin{array}{l}\text { Operating procedures } \\
\text { PPE }\end{array}$ & & E0 & $\mathrm{SD}$ \\
\hline PEP-30-F-02 & F2 & $\begin{array}{l}\text { Personnel injury } \\
\text { due to exposure to } \\
\text { process waste. }\end{array}$ & $\begin{array}{l}\text { Drop drum/tote filled with } \\
\text { process waste when } \\
\text { moving with forklift. } \\
\text { Spill contents inside } \\
\text { facility. }\end{array}$ & 1 & 3 & 4 & $\begin{array}{l}\text { Facility secondary containment } \\
\text { Personnel training and qualifications } \\
\text { Drum handling equipment on forklift } \\
\text { DOT containers }\end{array}$ & $\begin{array}{l}\text { Hoisting, Rigging, } \\
\text { Forklifts, and Aerial } \\
\text { Lifts Requirements } \\
\text { PPE }\end{array}$ & & E0 & $\mathrm{SD}$ \\
\hline PEP-30-F-03 & F2 & $\begin{array}{l}\text { Personnel injury } \\
\text { due to exposure to } \\
\text { process waste. }\end{array}$ & $\begin{array}{l}\text { Damage drum/tote filled } \\
\text { with process waste with } \\
\text { forklift. Spill contents } \\
\text { inside facility. }\end{array}$ & 1 & 3 & 4 & $\begin{array}{l}\text { Facility secondary containment } \\
\text { Personnel training and qualifications } \\
\text { Drum handling equipment on forklift } \\
\text { DOT containers }\end{array}$ & $\begin{array}{l}\text { Hoisting, Rigging, } \\
\text { Forklifts, and Aerial } \\
\text { Lifts Requirements } \\
\text { PPE }\end{array}$ & & E0 & $\mathrm{SD}$ \\
\hline PEP-30-F-04 & F2 & $\begin{array}{l}\text { Personnel injury } \\
\text { due to burn. }\end{array}$ & $\begin{array}{l}\text { Personnel exposure to } \\
\text { high temperature surface }\end{array}$ & 1 & 1 & 2 & $\begin{array}{l}\text { PPE (gloves, safety glasses) } \\
\text { Operating procedures }\end{array}$ & $\begin{array}{l}\text { PPE (gloves, safety } \\
\text { glasses) }\end{array}$ & & E0 & $\mathrm{SD}$ \\
\hline
\end{tabular}


Table E.1. Hazard Analysis Worksheets

\begin{tabular}{|c|c|c|c|c|c|c|c|c|c|c|c|}
\hline $\begin{array}{l}\text { Scenario } \\
\text { Number }\end{array}$ & Node & $\begin{array}{c}\text { Hazardous } \\
\text { Condition }\end{array}$ & Cause & $\mathbf{L}$ & C & $\begin{array}{c}\text { Risk } \\
\text { Score } \\
\end{array}$ & Potential Safeguard & $\begin{array}{c}\text { Credited } \\
\text { Safeguards } \\
\end{array}$ & $\begin{array}{c}\text { Comments/ } \\
\text { Assumptions }\end{array}$ & $\mathbf{E}$ & $\$$ \\
\hline & & & $\begin{array}{l}\text { due to drum/tote filled } \\
\text { with high-temperature } \\
\text { process waste. }\end{array}$ & & & & & Operating procedures & & & \\
\hline $\mathrm{n} / \mathrm{a}$ & F2 & & & & & & & & $\begin{array}{l}\text { Personnel injury due } \\
\text { to moving items with } \\
\text { forklift covered in } \\
\text { Node B. }\end{array}$ & & \\
\hline PEP-30-F-05 & $\mathrm{F} 2$ & $\begin{array}{l}\text { Personnel injury } \\
\text { due to exposure to } \\
\text { process waste. }\end{array}$ & $\begin{array}{l}\text { Drop drum/tote filled with } \\
\text { process waste when } \\
\text { moving over to the } \\
\text { storage pad. Spill } \\
\text { material outside of the } \\
\text { facility. }\end{array}$ & 1 & 2 & 3 & $\begin{array}{l}\text { Personnel training and qualifications } \\
\text { Drum handling equipment on forklift } \\
\text { DOT containers }\end{array}$ & $\begin{array}{l}\text { Hoisting, Rigging, } \\
\text { Forklifts, and Aerial } \\
\text { Lifts Requirements } \\
\text { PPE }\end{array}$ & & E1 & SD \\
\hline PEP-30-F-06 & F3 & $\begin{array}{l}\text { Personnel injury } \\
\text { due to exposure to } \\
\text { process waste. } \\
\text { Spill of drum/tote } \\
\text { contents to the } \\
\text { environment. }\end{array}$ & $\begin{array}{l}\text { Vehicle collision with } \\
90 \text {-day storage pad loaded } \\
\text { with drums/totes. }\end{array}$ & 0 & 4 & 4 & $\begin{array}{l}\text { PNNL speed limits } \\
\text { Emergency preparedness } \\
\text { Pad design for separation of chemical } \\
\text { types }\end{array}$ & \begin{tabular}{|l|} 
Speed limits (PNNL) \\
Restricted Access
\end{tabular} & & E2 & SD \\
\hline PEP-30-F-07 & F3 & $\begin{array}{l}\text { Personnel injury } \\
\text { due to exposure to } \\
\text { process waste. } \\
\text { Fire on pad. Spill } \\
\text { of drum/tote } \\
\text { contents to the } \\
\text { environment. } \\
\end{array}$ & $\begin{array}{l}\text { Vehicle collision with } \\
90 \text {-day storage pad loaded } \\
\text { with drums/totes. Fire } \\
\text { results from vehicle } \\
\text { accident. }\end{array}$ & -1 & 4 & 3 & $\begin{array}{l}\text { PNNL speed limits } \\
\text { Emergency preparedness } \\
\text { Pad design for separation of chemical } \\
\text { types }\end{array}$ & \begin{tabular}{|l} 
Speed limits (PNNL) \\
Restricted Access
\end{tabular} & & E2 & SD \\
\hline PEP-30-F-08 & F3 & $\begin{array}{l}\text { Personnel injury } \\
\text { due to exposure to } \\
\text { process waste. } \\
\text { Spill of drum/tote } \\
\text { contents on to the } \\
\text { pad. }\end{array}$ & $\begin{array}{l}\text { Low ambient } \\
\text { temperatures. Contents of } \\
\text { drum/totes freeze, } \\
\text { damaging container. Spill } \\
\text { contents when thaw. }\end{array}$ & 1 & 2 & 3 & $\begin{array}{l}\text { Pad secondary confinement } \\
\text { DOT containers } \\
\text { Pad design for separation of chemical } \\
\text { types }\end{array}$ & $\begin{array}{l}\text { 90-day storage pad } \\
\text { secondary } \\
\text { containment }\end{array}$ & & E0 & SD \\
\hline PEP-30-F-09 & F3 & $\begin{array}{l}\text { Personnel injury } \\
\text { due to exposure to } \\
\text { fumes. }\end{array}$ & $\begin{array}{l}\text { Additional nitric acid is } \\
\text { stored temporarily } \\
\text { outside. Spill of nitric } \\
\text { acid due to various } \\
\text { causes. Nitric pool } \\
\text { creates fumes. }\end{array}$ & 0 & 4 & 4 & \begin{tabular}{|l|} 
PNNL speed limits \\
Emergency preparedness \\
DOT containers \\
Personnel training and qualifications \\
Drum handling equipment on forklift
\end{tabular} & $\begin{array}{l}\text { Speed limits (PNNL) } \\
\text { Restricted Access } \\
\text { DOT containers for } \\
\text { Nitric }\end{array}$ & \begin{tabular}{|l} 
Transfer of addition \\
chemical inside \\
facility when needed \\
is covered under Node \\
B.
\end{tabular} & E0 & SD \\
\hline
\end{tabular}


Appendix F

Recommendations 
Table F.1. PEP Design Hazard Analysis Recommendations

\begin{tabular}{|c|c|c|c|c|c|}
\hline$\%$ & Recommendation $^{(a)}$ & Node & Issue(s) Addressed & Status & Comments \\
\hline $30-1$ & $\begin{array}{l}\text { Action: Assess tank systems } \\
\text { to determine whether a } \\
\text { damper in the ventilation } \\
\text { system is necessary. If yes, } \\
\text { consider safeguards: } \\
\text { - Change pressure indicator } \\
\text { to transmitting system } \\
\text { with alarm/interlock } \\
\text { Put a stop on the damper } \\
\text { to prevent full closure. }\end{array}$ & $\begin{array}{l}2,3 \\
5,6 \mathrm{a}\end{array}$ & $\begin{array}{l}\text { High pressure in } \\
\text { process tanks due } \\
\text { to blocked } \\
\text { ventilation (e.g., } \\
\text { damper closed) and } \\
\text { overfilling, } \\
\text { overheating, etc., } \\
\text { result in tank } \\
\text { failure. }\end{array}$ & $\begin{array}{l}\text { Closed. Damper in the } \\
\text { ventilation system is } \\
\text { necessary. Pressure } \\
\text { transmitter with AH \& } \\
\text { AHH added to tanks. } \\
\text { Tanks have vent for } \\
\text { passive air inlet, which } \\
\text { would prevent blocking in } \\
\text { tank. Also, ventilation } \\
\text { damper valves, modified } \\
\text { to prevent full closure, } \\
\text { were added to tanks } \\
\text { planned for heating to } \\
100^{\circ} \mathrm{C} \text {. }\end{array}$ & \\
\hline $30-2$ & $\begin{array}{l}\text { Action: Cleaning of process } \\
\text { vent will need to be analyzed } \\
\text { when the process is defined. }\end{array}$ & E3 & $\begin{array}{l}\text { Liquid intrusion } \\
\text { into ventilation } \\
\text { system }\end{array}$ & $\begin{array}{l}\text { Closed. Liquid build up } \\
\text { in the ventilation system } \\
\text { is prevented by steam } \\
\text { tracing and insulating the } \\
\text { ventilation line. In } \\
\text { addition, VNT-HTX T01 } \\
\text { was added to the off-gas } \\
\text { system, which will also } \\
\text { prevent buildup of liquid. }\end{array}$ & \\
\hline $30-3$ & $\begin{array}{l}\text { Action: Consider adding } \\
\text { liquid detector in vacuum } \\
\text { system }\end{array}$ & $\begin{array}{l}2 \mathrm{a} \\
3 \mathrm{a}\end{array}$ & $\begin{array}{l}\text { Vapor intrusion } \\
\text { into vacuum system }\end{array}$ & $\begin{array}{l}\text { Closed. Vacuum system } \\
\text { has cyclone separator to } \\
\text { remove liquid. Vacuum is } \\
\text { a liquid ring system, } \\
\text { which can tolerate some } \\
\text { liquid. }\end{array}$ & \\
\hline
\end{tabular}


Table F.1 (contd)

\begin{tabular}{|c|c|c|c|c|c|}
\hline$\%$ & Recommendation* & Node & Issue(s) Addressed & Status & Comments \\
\hline $\mid 30-4$ & $\begin{array}{l}\text { Action: Do analysis of how } \\
\text { the different materials will } \\
\text { respond to these } \\
\text { environmental conditions. }\end{array}$ & F3 & $\begin{array}{l}\text { Failure of material } \\
\text { containers due to } \\
\text { cold ambient } \\
\text { conditions releasing } \\
\text { hazardous material. }\end{array}$ & $\begin{array}{l}\text { Closed. Outdoor utilities } \\
\text { are designed for cold } \\
\text { weather (e.g., heat tracing } \\
\text { or additives to prevent } \\
\text { freezing). Outdoor } \\
\text { utilities and outdoor } \\
\text { chemical storage are } \\
\text { under the operational } \\
\text { auspices of PNNL F\&O } \\
\text { who will provide } \\
\text { personnel to monitor } \\
\text { systems and take } \\
\text { corrective actions to } \\
\text { prevent freezing when } \\
\text { conditions warrant (e.g., } \\
\text { lay up systems for cold } \\
\text { weather when systems are } \\
\text { idle). Indoor environment } \\
\text { will be maintained above } \\
\text { freezing. } \\
\end{array}$ & \\
\hline $30-5$ & $\begin{array}{l}\text { Action: Evaluate design for } \\
\text { heating and cooling tank. }\end{array}$ & $6 a$ & $\begin{array}{l}\text { Time required for } \\
\text { tank UFP-VSL- } \\
\text { T62A contents to } \\
\text { cool to desired } \\
\text { temperature for } \\
\text { material transfer } \\
\text { out. } \\
\end{array}$ & $\begin{array}{l}\text { Closed. Cooling coil } \\
\text { added to T62A. }\end{array}$ & \\
\hline $30-6$ & $\begin{array}{l}\text { Action: Need to evaluate } \\
\text { how material will respond } \\
\text { during cooling to determine } \\
\text { disposal packaging scheme } \\
\text { Action: Determine the } \\
\text { evaporation limits. }\end{array}$ & $6 a$ & \begin{tabular}{|l|} 
Over cooling or \\
excessive \\
evaporation \\
prevents transfer of \\
material out of \\
tank.
\end{tabular} & $\begin{array}{l}\text { Closed. Different } \\
\text { simulants will respond to } \\
\text { cooling differently. The } \\
\text { tank is equipped with } \\
\text { heating jacket, agitator, } \\
\text { water, and caustic } \\
\text { addition, which can be } \\
\text { manipulated to verify that } \\
\text { material can be } \\
\text { transported out of tank. } \\
\text { In general, simulant will } \\
\text { not be evaporated. Only } \\
\text { wash water is planned for } \\
\text { evaporation, and evapora- } \\
\text { tion limits have been set } \\
\text { at } 5 \text { molar sodium. }\end{array}$ & \\
\hline
\end{tabular}


Table F.1 (contd)

\begin{tabular}{|c|c|c|c|c|c|}
\hline$\%$ & Recommendation* & Node & Issue(s) Addressed & Status & Comments \\
\hline $30-7$ & $\begin{array}{l}\text { Action: Need to evaluate } \\
\text { how to treat the release of } \\
\text { excess steam in system. }\end{array}$ & 8 & $\begin{array}{l}\text { Sudden shutdown } \\
\text { of steam system } \\
\text { results in steam } \\
\text { release. }\end{array}$ & \begin{tabular}{|l|} 
Closed. If boiler is shut \\
down suddenly, excess \\
steam is diverted to the \\
blowdown drum via \\
pressure regulating valve \\
PRV-1323. Also pressure \\
safety valve PSV-1301 is \\
provided to protect boiler \\
vessel per code.
\end{tabular} & \\
\hline $30-8$ & $\begin{array}{l}\text { Action: Need to evaluate the } \\
\text { worker safety limits to see } \\
\text { how they are affected. }\end{array}$ & 7 & $\begin{array}{l}\text { Release from } \\
\text { ventilation system } \\
\text { prior to stack. }\end{array}$ & $\begin{array}{l}\text { Closed. Per ES\&H, } \\
\text { worker safety limits are } \\
\text { not expected to be } \\
\text { exceeded. In the event of } \\
\text { ventilation failure, staff } \\
\text { can close the passive air } \\
\text { inlet to tanks and verify } \\
\text { that the building forced- } \\
\text { air HVAC ventilation } \\
\text { system ( } 40,000 \text { SCFM) is } \\
\text { operating. }\end{array}$ & \\
\hline $30-9$ & $\begin{array}{l}\text { Action: Need to re-evaluate } \\
\text { this scenario during Phase } 3 \\
\text { integration. [No flow in the } \\
\text { system (damper closed), } \\
\text { blower failed, breaks in } \\
\text { system upstream of blower. } \\
\text { Any process vessel (T01A/B } \\
\text { and T02A) in operation vents } \\
\text { to manned facility spaces.] }\end{array}$ & 7 & $\begin{array}{l}\text { Venting of process } \\
\text { vessels into } \\
\text { manned spaces. }\end{array}$ & $\begin{array}{l}\text { Closed. Design has been } \\
\text { modified to vent all tanks } \\
\text { into off-gas system. }\end{array}$ & \\
\hline $30-10$ & $\begin{array}{l}\text { Action: Need to verify what } \\
\text { the prototypic design of the } \\
\text { heat exchanger is going to be } \\
\text { to define this event fully }\end{array}$ & 2 & $\begin{array}{l}\text { Low temperature in } \\
\text { process loop results } \\
\text { in plugging due to } \\
\text { solids precipitation }\end{array}$ & $\begin{array}{l}\text { Closed. The prototypic } \\
\text { design was available } \\
\text { during the } 90 \% \text { review, } \\
\text { and the HA was } \\
\text { completed. }\end{array}$ & \\
\hline $30-11$ & $\begin{array}{l}\text { Action: Perform calculation } \\
\text { to determine if enough } \\
\text { vacuum can be created by } \\
\text { this situation to cause feed } \\
\text { preparation tank damage. }\end{array}$ & $\begin{array}{l}1 \mathrm{a} \\
2,3 \\
5,6 \mathrm{a}\end{array}$ & $\begin{array}{l}\text { Process tanks } \\
\text { failure due to } \\
\text { excessive vacuum } \\
\text { in tank (excessive } \\
\text { cooling or empty- } \\
\text { ing with ventilation } \\
\text { blocked). }\end{array}$ & $\begin{array}{l}\text { Closed. This is no longer } \\
\text { a credible hazard as all } \\
\text { tanks have passive air } \\
\text { inlets and are equipped } \\
\text { with a stop or bore } \\
\text { through to prevent full } \\
\text { closure of ventilation. }\end{array}$ & \\
\hline $30-12$ & $\begin{array}{l}\text { Action: Consider venting } \\
\text { nitric addition tank to off-gas } \\
\text { system. }\end{array}$ & $4 \mathrm{a}$ & $\begin{array}{l}\text { Venting nitric acid } \\
\text { into manned } \\
\text { spaces. }\end{array}$ & $\begin{array}{l}\text { Closed. Nitric acid is } \\
\text { vented to the off-gas } \\
\text { system. }\end{array}$ & \\
\hline $30-13$ & $\begin{array}{l}\text { Add check valve on sodium } \\
\text { hydroxide line. }\end{array}$ & $6 \mathrm{a}$ & $\begin{array}{l}\text { Process tank } \\
\text { (UFP-VSL-T62A, } \\
\text { T62B) overfill due } \\
\text { to backflow from } \\
\text { other systems. }\end{array}$ & $\begin{array}{l}\text { Closed. Check valves } \\
\text { added. }\end{array}$ & \\
\hline
\end{tabular}


Table F.1 (Contd)

\begin{tabular}{|c|c|c|c|c|c|}
\hline$\%$ & Recommendation* & Node & $\begin{array}{c}\text { Issue(s) } \\
\text { Addressed }\end{array}$ & Status & Comments \\
\hline $30-14$ & $\begin{array}{l}\text { Add feature to deal with the } \\
\text { hold-up material in hoses at } \\
\text { completion of transfer. }\end{array}$ & B2 & $\begin{array}{l}\text { Release of } \\
\text { hazardous material } \\
\text { from flexible lines } \\
\text { after fill/drain } \\
\text { operations. }\end{array}$ & $\begin{array}{l}\text { Closed. Material will be } \\
\text { blown out of hoses with } \\
\text { air after transfer. }\end{array}$ & \\
\hline $30-15$ & $\begin{array}{l}\text { Add pressure relief on the } \\
\text { cooling jacket } \\
\text { Add low flow alarm } \\
\text { Action: Access the } \\
\begin{array}{l}\text { operating scheme with using } \\
\text { the temperature indicator on } \\
\text { the outlet of the system. }\end{array}\end{array}$ & 3 & $\begin{array}{l}\text { Failure of process } \\
\text { tank cooling jacket. }\end{array}$ & $\begin{array}{l}\text { Closed. Pressure relief } \\
\text { valve added to the design. }\end{array}$ & \\
\hline $30-16$ & $\begin{array}{l}\text { Add pressure relief on the } \\
\text { steam jacket. }\end{array}$ & $1 \mathrm{a}$ & $\begin{array}{l}\text { Failure of process } \\
\text { tank steam jacket. }\end{array}$ & $\begin{array}{l}\text { Closed. Pressure relief } \\
\text { valve added to the steam } \\
\text { jacket. }\end{array}$ & \\
\hline $30-17$ & $\begin{array}{l}\text { Add pressure relief to heat } \\
\text { exchanger. } \\
\text { Action: Perform calculation } \\
\text { to analyze event and design } \\
\text { piping to meet necessary } \\
\text { pressure rating. }\end{array}$ & $2,4 \mathrm{~b}$ & $\begin{array}{l}\text { Isolation of process } \\
\text { side of heat } \\
\text { exchanger with } \\
\text { continued steam } \\
\text { flow. }\end{array}$ & $\begin{array}{l}\text { Closed. PSV added to } \\
\text { relieve process side of } \\
\text { steam heat exchangers. } \\
\text { TKS indicated that pipe } \\
\text { rating of the filter loop } \\
\text { was increased to meet } \\
\text { pump design pressure. }\end{array}$ & \\
\hline $30-18$ & $\begin{array}{l}\text { Analytical capabilities } \\
\text { within PDL are yet to be } \\
\text { determined. These need to } \\
\text { be analyzed when defined. }\end{array}$ & $\mathrm{D} 2$ & $\begin{array}{l}\text { Identification of } \\
\text { potential personnel } \\
\text { safety issues } \\
\text { associated with } \\
\text { sample analysis. }\end{array}$ & $\begin{array}{l}\text { Closed. Analytical } \\
\text { capabilities in PDLW are } \\
\text { expected to include a } \\
\text { rheometer, centrifuge, } \\
\text { balance, and moisture } \\
\text { analyzer. These activities } \\
\text { were analyzed during the } \\
\text { final HA. }\end{array}$ & \\
\hline $30-19$ & $\begin{array}{l}\text { Change the design of feed } \\
\text { preparation and ultrafil- } \\
\text { tration tank systems to verify } \\
\text { that all potential leak paths } \\
\text { will leak to the secondary } \\
\text { confinement. }\end{array}$ & 2,3 & $\begin{array}{l}\text { Overfill of process } \\
\text { tanks (UFP-VSL- } \\
\text { T01 A/B, UFP- } \\
\text { VSL-T02A). }\end{array}$ & $\begin{array}{l}\text { Closed. The entire } \\
\text { PDLW floor is to serve as } \\
\text { secondary containment. }\end{array}$ & \\
\hline $30-20$ & $\begin{array}{l}\text { Consider adding a vehicle } \\
\text { barrier. }\end{array}$ & F3 & $\begin{array}{l}\text { Prevent vehicle } \\
\text { collisions with } \\
\text { material in tanks, } \\
\text { containers, and } \\
\text { facility. }\end{array}$ & $\begin{array}{l}\text { Closed. Fencing around } \\
\text { PDLW serves as a barrier } \\
\text { to unauthorized vehicle } \\
\text { traffic. Vehicle traffic is } \\
\text { to be controlled via the } \\
\text { Shipping and Receiving } \\
\text { procedure, which calls for } \\
\text { personnel to guide } \\
\text { vehicles and limits } \\
\text { vehicle speed. }\end{array}$ & \\
\hline
\end{tabular}


Table F.1 (Contd)

\begin{tabular}{|c|c|c|c|c|c|}
\hline$\%$ & Recommendation* & Node & $\begin{array}{c}\text { Issue(s) } \\
\text { Addressed }\end{array}$ & Status & Comments \\
\hline $30-21$ & $\begin{array}{l}\text { Consider designing bypass } \\
\text { for each of the ultrafilters. }\end{array}$ & 4 & $\begin{array}{l}\text { Loss of all } \\
\text { filtration capability } \\
\text { due to plugging of } \\
\text { one filter. }\end{array}$ & $\begin{array}{l}\text { Closed. Per direction of } \\
\text { BNI and ORP, a bypass } \\
\text { for each ultra filter will } \\
\text { not be added. }\end{array}$ & \\
\hline $30-22$ & $\begin{array}{l}\text { Consider how to isolate } \\
\text { portions of the steam system } \\
\text { to allow continued heating } \\
\text { when part of the system } \\
\text { needs to be open. }\end{array}$ & E2 & $\begin{array}{l}\text { Prevent } \\
\text { uncontrolled steam } \\
\text { release during } \\
\text { recovery/repair } \\
\text { activities. }\end{array}$ & $\begin{array}{l}\text { Closed. Double valve } \\
\text { protection added. }\end{array}$ & \\
\hline $30-23$ & $\begin{array}{l}\text { Consider means to support } \\
\text { ultrafiltration filters and } \\
\text { piping system during } \\
\text { replacement (so that holding } \\
\text { equipment by crane or } \\
\text { manually is not necessary } \\
\text { during removal/installation). }\end{array}$ & E4 & $\begin{array}{l}\text { Prevent load drop } \\
\text { during ultrafilter } \\
\text { replacement/repair. }\end{array}$ & $\begin{array}{l}\text { Closed. TKS presented } \\
\text { scheme where ultrafilters } \\
\text { would be changed with a } \\
\text { hoist and cart system. }\end{array}$ & \\
\hline $30-24$ & $\begin{array}{l}\text { Consider the need for PPE } \\
\text { (cooling vests). }\end{array}$ & A5 & $\begin{array}{l}\text { Reducing } \\
\text { personnel heat } \\
\text { stress in PDL. }\end{array}$ & $\begin{array}{l}\text { Closed. Heat stress } \\
\text { surveys will be taken } \\
\text { when conditions warrant, } \\
\text { and steps will be taken to } \\
\text { reduce heat stress to } \\
\text { support JHA. Options } \\
\text { include reducing length } \\
\text { of operating shift, } \\
\text { portable coolers, and } \\
\text { cooling vests. }\end{array}$ & $\begin{array}{l}\text { Additional PPE } \\
\text { defined in the } \\
\text { PDLW chemical } \\
\text { processing permits } \\
\text { and in the } \\
\text { procedures. }\end{array}$ \\
\hline $30-25$ & $\begin{array}{l}\text { Design of feed preparation } \\
\text { tank, receipt tank, and } \\
\text { ultrafiltration feed tank } \\
\text { PSVs must be considered for } \\
\text { operating in two phase } \\
\text { mode. }\end{array}$ & $\begin{array}{l}1 \mathrm{a}, 2, \\
3\end{array}$ & $\begin{array}{l}\text { Prevent process } \\
\text { tanks failure due to } \\
\text { overpressure in } \\
\text { tank. }\end{array}$ & $\begin{array}{l}\text { Closed. PSV were sized } \\
\text { in accordance with } \\
\text { ASME section VIII. } \\
\text { External fire was } \\
\text { determined by the } \\
\text { designer to be the worst } \\
\text { case scenario and was } \\
\text { used as the sizing basis } \\
\text { for the PSVs. }\end{array}$ & \\
\hline $30-26$ & $\begin{array}{l}\text { Design the package unit to } \\
\text { meet this potential } \\
\text { environment (liquid ring or } \\
\text { other suitable device, and } \\
\text { liquid detection). }\end{array}$ & 12 & $\begin{array}{l}\text { Prevent liquid } \\
\text { intrusion in } \\
\text { vacuum system. }\end{array}$ & $\begin{array}{l}\text { Closed. Vacuum system } \\
\text { has cyclone separator to } \\
\text { remove liquid. Vacuum } \\
\text { is a liquid ring system, } \\
\text { which can tolerate some } \\
\text { liquid. }\end{array}$ & \\
\hline $30-27$ & $\begin{array}{l}\text { Determine need for steam } \\
\text { trace on discharge and } \\
\text { stack. } \\
\text { Action: Need to re-evaluate } \\
\text { this scenario during Phase } 3 \\
\text { integration. }\end{array}$ & 7 & $\begin{array}{l}\text { Failure of } \\
\text { ventilation system } \\
\text { due to low } \\
\text { temperature } \\
\text { formation of } \\
\text { condensate. }\end{array}$ & $\begin{array}{l}\text { Closed. Steam trace } \\
\text { added to outside portion } \\
\text { of vent line. Insulation } \\
\text { added to stack. }\end{array}$ & \\
\hline
\end{tabular}


Table F.1 (Contd)

\begin{tabular}{|c|c|c|c|c|c|}
\hline$\%$ & Recommendation* & Node & $\begin{array}{c}\text { Issue(s) } \\
\text { Addressed }\end{array}$ & Status & Comments \\
\hline $30-28$ & Emergency lighting on skids. & $\begin{array}{l}\text { A1 } \\
\text { A5 }\end{array}$ & $\begin{array}{l}\text { Prevent personnel } \\
\text { mishap due to poor } \\
\text { visibility. }\end{array}$ & $\begin{array}{l}\text { Closed. TKS provided } \\
\text { outlets for emergency } \\
\text { lighting. PNNL to } \\
\text { procure and install } \\
\text { Emergency lighting as } \\
\text { necessary. }\end{array}$ & \\
\hline $30-29$ & $\begin{array}{l}\text { Verify that the sample } \\
\text { container is designed for } \\
\text { high temperatures. }\end{array}$ & D1 & $\begin{array}{l}\text { Prevent sample } \\
\text { container failure } \\
\text { due to high } \\
\text { temperature } \\
\text { sample. }\end{array}$ & $\begin{array}{l}\text { Closed. Sample } \\
\text { containers will be } \\
\text { polypropylene designed } \\
\text { for high temperatures } \\
\left(100^{\circ} \mathrm{C}\right) .\end{array}$ & \\
\hline $30-30$ & $\begin{array}{l}\text { Evaluate redesign of the } \\
\text { vent system to minimize } \\
\text { possibility of plugging the } \\
\text { vent. }\end{array}$ & 9 & $\begin{array}{l}\text { Prevent tank vent } \\
\text { plugging. }\end{array}$ & $\begin{array}{l}\text { Closed. Cleaning ports } \\
\text { added to all knock out } \\
\text { pots on top of tanks. } \\
\text { System designed to } \\
\text { handle expected water } \\
\text { vapor. }\end{array}$ & \\
\hline $30-31$ & $\begin{array}{l}\text { Include standoffs for tops of } \\
\text { tanks without top insulation. }\end{array}$ & A1 & $\begin{array}{l}\text { Prevent personnel } \\
\text { exposure to high } \\
\text { temperature } \\
\text { surfaces. }\end{array}$ & $\begin{array}{l}\text { Closed. Tanks above } \\
140^{\circ} \mathrm{F} \text { will have insula- } \\
\text { tion. Tanks T01A/B and } \\
\text { T02 will have removable } \\
\text { insulation. Personnel will } \\
\text { have to wear appropriate } \\
\text { PPE if removal insulation } \\
\text { is off. }\end{array}$ & \\
\hline $30-32$ & $\begin{array}{l}\text { Manual handling of } \\
\text { drums/totes will require } \\
\text { specific PPE. }\end{array}$ & $\mathrm{F} 2$ & $\begin{array}{l}\text { Protect personnel } \\
\text { from high } \\
\text { temperature and } \\
\text { material exposure } \\
\text { hazards. }\end{array}$ & $\begin{array}{l}\text { Closed. PPE defined in } \\
\text { the PDLW chemical } \\
\text { processing permits and in } \\
\text { the procedures. }\end{array}$ & \\
\hline $30-33$ & $\begin{array}{l}\text { Measure totalized volume of } \\
\text { steam on each ejector. }\end{array}$ & $3 b$ & $\begin{array}{l}\text { Dilution of process } \\
\text { batch due to excess } \\
\text { steam condensate. }\end{array}$ & $\begin{array}{l}\text { Closed. Each ejector has } \\
\text { a steam flow meter. }\end{array}$ & $\begin{array}{l}\text { Lost test time } \\
\text { issue. }\end{array}$ \\
\hline $30-34$ & $\begin{array}{l}\text { Procedurally use flow } \\
\text { totalizer to monitor the } \\
\text { material addition. }\end{array}$ & $1 \mathrm{a}$ & $\begin{array}{l}\text { "Out of spec" batch } \\
\text { due to valve } \\
\text { misalignment. }\end{array}$ & $\begin{array}{l}\text { Closed. Flow totalizers } \\
\text { are used in the procedure } \\
\text { to monitor material } \\
\text { addition. }\end{array}$ & $\begin{array}{l}\text { Lost test time } \\
\text { issue. }\end{array}$ \\
\hline $30-35$ & $\begin{array}{l}\text { Procedurally use level } \\
\text { detection and flow totalizer } \\
\text { to monitor the material } \\
\text { addition. }\end{array}$ & $\begin{array}{l}1 \mathrm{a}, 2 \\
3,5 \\
6 \mathrm{a}\end{array}$ & $\begin{array}{l}\text { "Out of spec" batch } \\
\text { due to incorrect } \\
\text { mass additions to } \\
\text { process tanks. }\end{array}$ & $\begin{array}{l}\text { Closed. Level indicators } \\
\text { and flow totalizers are } \\
\text { used in the procedure to } \\
\text { monitor material } \\
\text { addition. }\end{array}$ & $\begin{array}{l}\text { Lost test time } \\
\text { issue. }\end{array}$ \\
\hline $30-36$ & $\begin{array}{l}\text { Provide interlock on firing } \\
\text { of pulse pots to confirm } \\
\text { pressure. }\end{array}$ & 11 & $\begin{array}{l}\text { Prevent backflow } \\
\text { of permeate into } \\
\text { compressed air } \\
\text { system. }\end{array}$ & $\begin{array}{l}\text { Closed. Logic calls for } \\
\text { pulse pots to be vented to } \\
\text { remove some permeate in } \\
\text { pulse pot immediately } \\
\text { prior to charging pulse } \\
\text { pot with air. }\end{array}$ & \\
\hline
\end{tabular}


Table F.1 (Contd)

\begin{tabular}{|c|c|c|c|c|c|}
\hline$\%$ & Recommendation* & Node & $\begin{array}{c}\text { Issue(s) } \\
\text { Addressed }\end{array}$ & Status & Comments \\
\hline $30-37$ & $\begin{array}{l}\text { Require raw material } \\
\text { sample/ test upon receipt to } \\
\text { verify that the material is in } \\
\text { spec. }\end{array}$ & $\begin{array}{l}1 \mathrm{a}, 2 \\
3\end{array}$ & $\begin{array}{l}\text { "Out of spec" batch } \\
\text { due to "out of } \\
\text { spec" addition. }\end{array}$ & $\begin{array}{l}\text { Closed. Procedures call } \\
\text { for sample of raw } \\
\text { material upon receipt at } \\
\text { the tanker and for } \\
\text { periodic sampling of the } \\
\text { chemical addition tanks. }\end{array}$ & $\begin{array}{l}\text { Lost test time } \\
\text { issue. }\end{array}$ \\
\hline $30-38$ & $\begin{array}{l}\text { Require raw material } \\
\text { sample/test upon receipt to } \\
\text { verify that the material is in } \\
\text { spec. } \\
\text { Evaluate the sampling port } \\
\text { location on the chemical } \\
\text { addition tanks. }\end{array}$ & 9 & $\begin{array}{l}\text { "Out of spec" batch } \\
\text { due to "out of } \\
\text { spec" addition. }\end{array}$ & $\begin{array}{l}\text { Closed. TKS has } \\
\text { provided sampling ports } \\
\text { on chemical addition } \\
\text { tanks. }\end{array}$ & $\begin{array}{l}\text { TKS will design } \\
\text { the sampling to } \\
\text { meet best practice. }\end{array}$ \\
\hline $30-39$ & $\begin{array}{l}\text { The shake-down test plan } \\
\text { (Node E1) will adequately } \\
\text { address the safety hazards } \\
\text { associated with the plan. } \\
\text { The plan should be } \\
\text { reviewed, when developed, } \\
\text { for its consistency with the } \\
\text { developed hazard analysis. }\end{array}$ & E1 & $\begin{array}{l}\text { Management of } \\
\text { personnel hazards } \\
\text { during installation } \\
\text { of PEP in PDL } \\
\text { West. }\end{array}$ & $\begin{array}{l}\text { Closed. PEP Safeguards } \\
\text { that consist of equipment } \\
\text { will be tested during } \\
\text { shakedown testing. } \\
\text { Safeguards for hazardous } \\
\text { conditions with a risk } \\
\text { score of }>3 \text { will be "full } \\
\text { loop" tested. Those for } \\
\text { conditions with scores } 3 \\
\text { or less will be tested to } \\
\text { verify operability. }\end{array}$ & $\begin{array}{l}\text { Draft shakedown } \\
\text { plan has been } \\
\text { developed and will } \\
\text { be reviewed. }\end{array}$ \\
\hline $30-40$ & $\begin{array}{l}\text { TKS will design the } \\
\text { sampling to meet best } \\
\text { practice. The activities } \\
\text { associated with sampling in } \\
\text { the facility will be addressed } \\
\text { during the phase } 3 \\
\text { integration analysis. }\end{array}$ & 13 & $\begin{array}{l}\text { Prevention of } \\
\text { uncontrolled } \\
\text { releases of } \\
\text { hazardous material } \\
\text { during sampling. }\end{array}$ & $\begin{array}{l}\text { Closed. Best practice has } \\
\text { been incorporated in } \\
\text { permeate sampling } \\
\text { system (missing local } \\
\text { pressure gauge). Also } \\
\text { sampler input not } \\
\text { reflected for in-line slurry } \\
\text { samples. }\end{array}$ & $\begin{array}{l}\text { Analyzed in } \\
\text { Phase } 3 \text { as Node D. }\end{array}$ \\
\hline $90-1$ & $\begin{array}{l}\text { Action: Provide safeguard } \\
\text { (e.g., PSV) if design } \\
\text { pressure for the filter } \\
\text { assembly is less than } \\
\text { maximum air pressure. }\end{array}$ & $4 a$ & $\begin{array}{l}\text { Prevention of } \\
\text { overpressure } \\
\text { failure of } \\
\text { ultrafilter. }\end{array}$ & $\begin{array}{l}\text { Closed. Maximum } \\
\text { allowable working } \\
\text { pressure for the shell per } \\
\text { Mott drawings ( } \# 7300018 \\
\text { Rev. 3) is } 482 \text { psig, } \\
\text { which matches the } \\
\text { maximum planned air } \\
\text { pressure. }\end{array}$ & \\
\hline $90-2$ & $\begin{array}{l}\text { Add pressure relief on the } \\
\text { steam jacket. }\end{array}$ & $6 a$ & $\begin{array}{l}\text { Prevent failure of } \\
\text { steam jacket on } \\
\text { collection tank. }\end{array}$ & $\begin{array}{l}\text { Closed. PSV was added } \\
\text { to steam jacket on tank } \\
\text { UFP-VSL-62A in Rev. } 4 \\
\text { P\&IDs. }\end{array}$ & \\
\hline
\end{tabular}


Table F.1 (Contd)

\begin{tabular}{|c|c|c|c|c|c|}
\hline$\%$ & Recommendation* & Node & $\begin{array}{c}\text { Issue(s) } \\
\text { Addressed }\end{array}$ & Status & Comments \\
\hline $90-3$ & $\begin{array}{l}\text { Action: Need to have means } \\
\text { to close passive vents in the } \\
\text { event of ventilation system } \\
\text { failure. }\end{array}$ & 7 & $\begin{array}{l}\text { Prevent vapor } \\
\text { releases into } \\
\text { manned spaces } \\
\text { when vent system } \\
\text { shuts down. }\end{array}$ & $\begin{array}{l}\text { Closed. P\&IDs show } \\
\text { blind flange for each } \\
\text { passive vent that could be } \\
\text { closed in the event of } \\
\text { ventilation failure. }\end{array}$ & \\
\hline $90-4$ & $\begin{array}{l}\text { Regular monitoring of } \\
\text { glycol concentration in } \\
\text { chilled water tank. }\end{array}$ & 10 & $\begin{array}{l}\text { Prevent loss of } \\
\text { chilling capabilities } \\
\text { and damage to } \\
\text { system due to low } \\
\text { ambient } \\
\text { temperatures. }\end{array}$ & $\begin{array}{l}\text { Closed. TKS provided } \\
\text { means to sample chill } \\
\text { water system in Rev. } 4 \\
\text { P\&IDs. PNNL F\&O will } \\
\text { monitor glycol } \\
\text { concentration of chill } \\
\text { water system. }\end{array}$ & \\
\hline $90-5$ & $\begin{array}{l}\text { Pressure regulating valve } \\
\text { upstream of amplifier (being } \\
\text { added). }\end{array}$ & 11 & $\begin{array}{l}\text { Prevent failure of } \\
\text { down stream } \\
\text { components due to } \\
\text { high pressure } \\
\text { compressed air. }\end{array}$ & $\begin{array}{l}\text { Closed. PSV added in } \\
\text { Rev. } 4 \text { P\&IDs. }\end{array}$ & \\
\hline
\end{tabular}


Appendix G

Safeguard Allocation 
Table G-1. Safeguard Allocation

\begin{tabular}{|c|c|c|c|c|c|}
\hline $\begin{array}{l}\text { Scenario } \\
\text { Number }\end{array}$ & $\begin{array}{l}\text { No } \\
\text { de }\end{array}$ & Hazardous Condition & Cause & $\begin{array}{l}\text { Risk } \\
\text { Score }\end{array}$ & Credited Safeguards \\
\hline PEP-30-1-02 & $1 \mathrm{a}$ & $\begin{array}{l}\text { Spill of simulant } \\
\text { (inside or outside } \\
\text { facility). }\end{array}$ & $\begin{array}{l}\text { No flow into the receipt tank } \\
\text { due to leak at the connection } \\
\text { to the simulant addition flex } \\
\text { hose. }\end{array}$ & 5 & $\begin{array}{l}\text { PPE } \\
\text { Operating procedures } \\
\text { Facility secondary } \\
\text { containment } \\
\text { Receipt tank level } \\
\text { detection }\end{array}$ \\
\hline PEP-30-1-03 & $1 \mathrm{a}$ & $\begin{array}{l}\text { Spill of simulant, } \\
\text { sodium hydroxide, } \\
\text { material from other } \\
\text { tanks or water. }\end{array}$ & $\begin{array}{l}\text { No flow into the receipt tank } \\
\text { due to leak in simulant } \\
\text { addition or transfer piping } \\
\text { (flanges, etc.). }\end{array}$ & 4 & $\begin{array}{l}\text { PPE } \\
\text { Operating procedures } \\
\text { Facility secondary } \\
\text { containment } \\
\text { Receipt tank level } \\
\text { detection }\end{array}$ \\
\hline PEP-30-1-06 & $1 \mathrm{a}$ & $\begin{array}{l}\text { Spill of simulant, } \\
\text { sodium hydroxide, } \\
\text { material from other } \\
\text { tanks or water. }\end{array}$ & $\begin{array}{l}\text { Structural integrity of receipt } \\
\text { tank degraded, resulting in } \\
\text { leak. }\end{array}$ & 1 & $\begin{array}{l}\text { Design of receipt } \\
\text { tanks to code }\end{array}$ \\
\hline PEP-30-1-07 & $1 \mathrm{a}$ & $\begin{array}{l}\text { Spill of simulant, } \\
\text { sodium hydroxide, } \\
\text { material, or water from } \\
\text { other tanks. }\end{array}$ & $\begin{array}{l}\text { No flow out of the receipt } \\
\text { tank due to leak in transfer } \\
\text { piping/pumps. }\end{array}$ & 4 & $\begin{array}{l}\text { PPE } \\
\text { Operating procedures } \\
\text { Facility secondary } \\
\text { containment } \\
\text { Receipt tank level } \\
\text { detection }\end{array}$ \\
\hline PEP-30-1-12 & $1 \mathrm{a}$ & $\begin{array}{l}\text { Overflow of tank } \\
\text { contents to ventilation } \\
\text { system, damaging fans, } \\
\text { exchanger, and blower. } \\
\text { Boil off liquids forming } \\
\text { solids in vent lines. }\end{array}$ & $\begin{array}{l}\text { Too much material added to } \\
\text { the receipt tank, resulting in } \\
\text { overflow of tank with valves } \\
\text { closed. }\end{array}$ & 2 & $\begin{array}{l}\text { Receipt tank level } \\
\text { detection and alarms }\end{array}$ \\
\hline PEP-30-1-13 & $1 \mathrm{a}$ & $\begin{array}{l}\text { Incomplete/"out of } \\
\text { spec" batch of material. } \\
\text { Lost time for test. }\end{array}$ & $\begin{array}{l}\text { Too much material added to } \\
\text { the receipt tank. High level in } \\
\text { tank. }\end{array}$ & 3 & $\begin{array}{l}\text { Receipt tank level } \\
\text { detection and alarms }\end{array}$ \\
\hline PEP-30-1-15 & $1 \mathrm{a}$ & $\begin{array}{l}\text { Incomplete/“out of } \\
\text { spec" batch of material. } \\
\text { Lost time for test. }\end{array}$ & $\begin{array}{l}\text { Too little caustic added to } \\
\text { receipt tank resulting in Tank } \\
\text { UFP-VSL-T01A/B contents } \\
\text { too low in caustic. }\end{array}$ & 3 & $\begin{array}{l}\text { Receipt tank level } \\
\text { detection }\end{array}$ \\
\hline PEP-30-1-16 & $1 \mathrm{a}$ & $\begin{array}{l}\text { Incomplete/"out of } \\
\text { spec" batch of material. } \\
\text { Lost time for test. }\end{array}$ & $\begin{array}{l}\text { Delivery of "out of spec" } \\
\text { caustic or simulant results in } \\
\text { wrong batch specs. }\end{array}$ & 3 & Operating procedures \\
\hline PEP-30-1-17 & $1 \mathrm{a}$ & $\begin{array}{l}\text { Incomplete/"out of } \\
\text { spec" batch of material. } \\
\text { Lost time for test. }\end{array}$ & $\begin{array}{l}\text { Incorrect valve lineup } \\
\text { between receipt tanks creates } \\
\text { a misbatching. }\end{array}$ & 3 & Operating procedures \\
\hline PEP-30-1-18 & $1 \mathrm{a}$ & $\begin{array}{l}\text { Overflow of receipt } \\
\text { tank contents to } \\
\text { ventilation system, } \\
\text { damaging fans, } \\
\text { exchanger, and blower. } \\
\text { Boil off liquids forming } \\
\text { solids in vent lines. }\end{array}$ & $\begin{array}{l}\text { Backflow of material from } \\
\text { UFP-VSL-Y01A/B to receipt } \\
\text { tank caused by transfer of } \\
\text { material into A/B overflowing } \\
\text { back. }\end{array}$ & 3 & $\begin{array}{l}\text { Receipt tank level } \\
\text { detection and alarms }\end{array}$ \\
\hline
\end{tabular}


Table G-1. Safeguard Allocation

\begin{tabular}{|c|c|c|c|c|c|}
\hline $\begin{array}{l}\text { Scenario } \\
\text { Number }\end{array}$ & $\begin{array}{l}\text { No } \\
\text { de }\end{array}$ & Hazardous Condition & Cause & $\begin{array}{l}\text { Risk } \\
\text { Score }\end{array}$ & Credited Safeguards \\
\hline PEP-30-1-21 & 1a & $\begin{array}{l}\text { Failure of tank and } \\
\text { steam jacket and spill } \\
\text { of tank contents. }\end{array}$ & $\begin{array}{l}\text { High pressure in receipt tank } \\
\text { due to ventilation blocked } \\
\text { (closure of damper) and } \\
\text { overfilling of tank. }\end{array}$ & 6 & $\begin{array}{l}\text { PSV on receipt tank } \\
\text { Stop or bore through } \\
\text { on the damper to } \\
\text { prevent full closure. }\end{array}$ \\
\hline PEP-30-1-22 & $1 \mathrm{a}$ & $\begin{array}{l}\text { Failure of tank and } \\
\text { steam jacket and spill } \\
\text { of tank contents. }\end{array}$ & $\begin{array}{l}\text { High pressure in receipt tank } \\
\text { due to ventilation blocked } \\
\text { (closure of damper) and } \\
\text { overheat the tank due to } \\
\text { temperature control system } \\
\text { failure. }\end{array}$ & 4 & $\begin{array}{l}\text { PSV on receipt tank } \\
\text { Stop or bore through } \\
\text { on the damper to } \\
\text { prevent full closure. }\end{array}$ \\
\hline PEP-30-1-30 & $1 \mathrm{a}$ & $\begin{array}{l}\text { Small release of steam } \\
\text { to the facility. }\end{array}$ & $\begin{array}{l}\text { Structural failure of the steam } \\
\text { jacket on receipt tank. Small } \\
\text { leak of steam. }\end{array}$ & 1 & $\begin{array}{l}\text { PSV on the steam } \\
\text { jacket }\end{array}$ \\
\hline PEP-30-1-31 & $1 \mathrm{a}$ & $\begin{array}{l}\text { Large release of steam } \\
\text { to the facility. }\end{array}$ & $\begin{array}{l}\text { Catastrophic failure of the } \\
\text { steam jacket on receipt tank } \\
\text { results in large release of } \\
\text { steam. }\end{array}$ & 2 & $\begin{array}{l}\text { PSV on the steam } \\
\text { jacket }\end{array}$ \\
\hline PEP-30-1-32 & $1 \mathrm{a}$ & $\begin{array}{l}\text { Collapse of steam } \\
\text { jacket. }\end{array}$ & $\begin{array}{l}\text { Steam present in the steam } \\
\text { jacket on the receipt tank. } \\
\text { Block off jacket; add cold } \\
\text { water to refill. Vacuum } \\
\text { pulled collapsing jacket. }\end{array}$ & 2 & $\begin{array}{l}\text { Vacuum breaker on } \\
\text { steam jacket }\end{array}$ \\
\hline PEP-30-2-02 & 2 & $\begin{array}{l}\text { Spill of simulant, } \\
\text { sodium hydroxide, } \\
\text { material, or inhibited } \\
\text { water from other tanks. }\end{array}$ & $\begin{array}{l}\text { No flow into the feed } \\
\text { preparation tank due to leak } \\
\text { in chemical addition or } \\
\text { transfer piping (flanges, etc.). }\end{array}$ & 4 & $\begin{array}{l}\text { PPE } \\
\text { Operating procedures } \\
\text { Facility secondary } \\
\text { containment } \\
\text { Feed Preparation tank } \\
\text { level detection } \\
\end{array}$ \\
\hline PEP-30-2-06 & 2 & Spill of tank contents. & $\begin{array}{l}\text { Structural integrity of feed } \\
\text { preparation tank degraded } \\
\text { resulting in leak. }\end{array}$ & 1 & $\begin{array}{l}\text { Design of feed } \\
\text { preparation tanks to } \\
\text { code }\end{array}$ \\
\hline PEP-30-2-07 & 2 & Spill of tank contents. & $\begin{array}{l}\text { No flow out of the feed } \\
\text { preparation tank due to leak } \\
\text { in transfer piping/pumps. }\end{array}$ & 4 & $\begin{array}{l}\text { PPE } \\
\text { Operating procedures } \\
\text { Facility secondary } \\
\text { containment } \\
\text { Feed Preparation tank } \\
\text { level detection }\end{array}$ \\
\hline PEP-30-2-08 & 2 & $\begin{array}{l}\text { Carryover of material } \\
\text { to the ventilation } \\
\text { system. }\end{array}$ & $\begin{array}{l}\text { Lower-than-intended feed } \\
\text { preparation tank volume. } \\
\text { Level in tank below level of } \\
\text { the PJMs results in overblow. } \\
\text { Material in the vent line. }\end{array}$ & 3 & $\begin{array}{l}\text { PLC } \\
\text { PJM Level Detector } \\
\text { PJM Pressure } \\
\text { Detector } \\
\text { PJM Rack Air Supply } \\
\text { Valve } \\
\text { Feed Preparation tank } \\
\text { level detection }\end{array}$ \\
\hline PEP-30-2-11 & 2 & $\begin{array}{l}\text { Spill tank contents to } \\
\text { floor. }\end{array}$ & $\begin{array}{l}\text { Too much material added to } \\
\text { the feed preparation tank } \\
\text { resulting in overflow of tank } \\
\text { to facility floor. }\end{array}$ & 4 & $\begin{array}{l}\text { PPE } \\
\text { Operating procedures } \\
\text { Facility secondary } \\
\text { containment } \\
\text { Feed Preparation tank } \\
\text { level detection and } \\
\end{array}$ \\
\hline
\end{tabular}


Table G-1. Safeguard Allocation

\begin{tabular}{|c|c|c|c|c|c|}
\hline $\begin{array}{l}\text { Scenario } \\
\text { Number }\end{array}$ & $\begin{array}{l}\text { No } \\
\text { de }\end{array}$ & Hazardous Condition & Cause & $\begin{array}{l}\text { Risk } \\
\text { Score }\end{array}$ & Credited Safeguards \\
\hline & & & & & alarms \\
\hline PEP-30-2-12 & 2 & $\begin{array}{l}\text { Incomplete/"out of } \\
\text { spec" batch of material. } \\
\text { Lost time for test. }\end{array}$ & $\begin{array}{l}\text { Too much material added to } \\
\text { the feed preparation tank. } \\
\text { High level in tank. }\end{array}$ & 3 & $\begin{array}{l}\text { Feed Preparation tank } \\
\text { level detection and } \\
\text { alarms }\end{array}$ \\
\hline PEP-30-2-14 & 2 & $\begin{array}{l}\text { Incomplete/“out of } \\
\text { spec" batch of material. } \\
\text { Lost time for test. }\end{array}$ & $\begin{array}{l}\text { Too little caustic added to the } \\
\text { feed preparation tank results } \\
\text { in Tank UFP-VSL-T02A } \\
\text { caustic concentration being } \\
\text { too low. }\end{array}$ & 3 & Operating procedures \\
\hline PEP-30-2-15 & 2 & $\begin{array}{l}\text { Incomplete/"out of } \\
\text { spec" batch of material. } \\
\text { Lost time for test. }\end{array}$ & $\begin{array}{l}\text { Delivery of "out of spec" } \\
\text { caustic results in wrong batch } \\
\text { specs. }\end{array}$ & 3 & Operating procedures \\
\hline PEP-30-2-18 & 2 & $\begin{array}{l}\text { Failure of tank and spill } \\
\text { of tank contents. }\end{array}$ & $\begin{array}{l}\text { High pressure in feed } \\
\text { preparation tank due to } \\
\text { ventilation blocked (closure } \\
\text { of damper, plugged) and } \\
\text { overfilling of tank. }\end{array}$ & 3 & $\begin{array}{l}\text { PSV on Feed } \\
\text { Preparation tank } \\
\text { Stop or bore through } \\
\text { on the damper to } \\
\text { prevent full closure. }\end{array}$ \\
\hline PEP-30-2-19 & 2 & $\begin{array}{l}\text { Failure of tank and spill } \\
\text { of tank contents. }\end{array}$ & $\begin{array}{l}\text { High pressure in feed } \\
\text { preparation tank due to } \\
\text { ventilation blocked (closure } \\
\text { of damper, plugged) and } \\
\text { overheat the tank due to } \\
\text { temperature control system } \\
\text { failure. }\end{array}$ & 4 & $\begin{array}{l}\text { PSV on Feed } \\
\text { Preparation tank } \\
\text { Stop or bore through } \\
\text { on the damper to } \\
\text { prevent full closure. }\end{array}$ \\
\hline PEP-30-2-27 & 2 & $\begin{array}{l}\text { Contaminate/damage } \\
\text { the chilled water or } \\
\text { steam supply system. }\end{array}$ & $\begin{array}{l}\text { Failure in heat exchanger } \\
\text { causes leak of process } \\
\text { material into chilled water or } \\
\text { steam supply. Simulant } \\
\text { mixture plugs system. }\end{array}$ & 1 & Operating procedures \\
\hline PEP-30-2-30 & 2 & $\begin{array}{l}\text { Damage the heat } \\
\text { exchanger loop, } \\
\text { releasing heated } \\
\text { process material. }\end{array}$ & $\begin{array}{l}\text { Closing isolation valves to } \\
\text { heat exchanger with } \\
\text { continued heating causes over } \\
\text { pressurization. }\end{array}$ & 6 & $\begin{array}{l}\text { Operating procedures } \\
\text { PSV on heat } \\
\text { exchanger }\end{array}$ \\
\hline PEP-30-2-31 & 2 & $\begin{array}{l}\text { Large release steam to } \\
\text { the facility. }\end{array}$ & $\begin{array}{l}\text { Catastrophic failure of the } \\
\text { steam shell on the heat } \\
\text { exchanger results in large } \\
\text { release of steam. }\end{array}$ & 2 & $\begin{array}{l}\text { Design of steam shell } \\
\text { to code }\end{array}$ \\
\hline PEP-30-2-32 & 2 & Collapse of steam shell. & $\begin{array}{l}\text { Steam present in the shell, } \\
\text { blocked off. Vacuum pulled } \\
\text { collapsing shell upon cooling. }\end{array}$ & 2 & Operating procedures \\
\hline PEP-90-2-01 & 2 & $\begin{array}{l}\text { Spill of simulant, } \\
\text { sodium hydroxide, } \\
\text { material, or inhibited } \\
\text { water from other tanks, } \\
\text { to the facility. }\end{array}$ & $\begin{array}{l}\text { Flush valves are open during } \\
\text { transfer, spilling material to } \\
\text { the facility. }\end{array}$ & 4 & $\begin{array}{l}\text { PPE } \\
\text { Facility secondary } \\
\text { containment } \\
\text { Operating procedures } \\
\text { Feed Preparation tank } \\
\text { level detection }\end{array}$ \\
\hline PEP-90-2-03 & 2 & $\begin{array}{l}\text { Spill of simulant, } \\
\text { sodium hydroxide, } \\
\text { material, or inhibited } \\
\text { water from other tanks, } \\
\text { to the facility. }\end{array}$ & $\begin{array}{l}\text { Control valve is mis-aligned } \\
\text { during recirculation, resulting } \\
\text { in material traveling to } \\
\text { ultrafiltration feed vessel } \\
\text { (T02A). Tank overflows to }\end{array}$ & 3 & $\begin{array}{l}\text { PPE } \\
\text { Facility secondary } \\
\text { containment } \\
\text { Operating procedures }\end{array}$ \\
\hline
\end{tabular}


Table G-1. Safeguard Allocation

\begin{tabular}{|c|c|c|c|c|c|}
\hline $\begin{array}{l}\text { Scenario } \\
\text { Number }\end{array}$ & $\begin{array}{l}\text { No } \\
\text { de }\end{array}$ & Hazardous Condition & Cause & $\begin{array}{l}\text { Risk } \\
\text { Score }\end{array}$ & Credited Safeguards \\
\hline & & & facility. & & \\
\hline PEP-90-2-04 & $2 a$ & $\begin{array}{l}\text { Personnel injury due to } \\
\text { debris projectiles. }\end{array}$ & $\begin{array}{l}\text { Feeding PJMs with } 100 \mathrm{psi} \\
\text { air, open vacuum valve, and } \\
\text { overpressurize the vacuum } \\
\text { system. Failure of vacuum } \\
\text { system. }\end{array}$ & 4 & $\begin{array}{l}\text { PLC } \\
\text { PJM Level Detector } \\
\text { PJM Pressure } \\
\text { Detector } \\
\text { PJM Rack Air Supply } \\
\text { Valve } \\
\text { Feed Preparation tank } \\
\text { level detection }\end{array}$ \\
\hline PEP-30-2-34 & $2 b$ & $\begin{array}{l}\text { Incomplete/“out of } \\
\text { spec” batch of material. } \\
\text { Lost time for test. }\end{array}$ & $\begin{array}{l}\text { Add too much water to the } \\
\text { feed preparation tank } \\
\text { (inhibited or condensate from } \\
\text { the sparger). }\end{array}$ & 3 & $\begin{array}{l}\text { Operating procedures } \\
\text { Feed Preparation tank } \\
\text { level detection }\end{array}$ \\
\hline PEP-30-2-36 & $2 b$ & $\begin{array}{l}\text { Large steam leak } \\
\text { outside of feed } \\
\text { preparation tank. }\end{array}$ & $\begin{array}{l}\text { Failure of the steam system } \\
\text { upstream of the tank resulting } \\
\text { in large release of steam. }\end{array}$ & 2 & $\begin{array}{l}\text { Design of steam } \\
\text { system to code } \\
\text { Operating procedures }\end{array}$ \\
\hline PEP-30-2-37 & $2 b$ & $\begin{array}{l}\text { Small steam leak } \\
\text { outside of feed } \\
\text { preparation tank. }\end{array}$ & $\begin{array}{l}\text { Failure of the steam system } \\
\text { upstream of the tank resulting } \\
\text { in small release of steam. }\end{array}$ & 2 & $\begin{array}{l}\text { Design of steam } \\
\text { system to code } \\
\text { Operating procedures }\end{array}$ \\
\hline PEP-30-2-41 & $2 a$ & $\begin{array}{l}\text { Carryover of material } \\
\text { to the ventilation } \\
\text { system. }\end{array}$ & $\begin{array}{l}\text { High flow through the PJMs } \\
\text { results in overblow. }\end{array}$ & 3 & $\begin{array}{l}\text { PLC } \\
\text { PJM Level Detector } \\
\text { PJM Pressure } \\
\text { Detector } \\
\text { PJM Rack Air Supply } \\
\text { Valve } \\
\text { Feed Preparation tank } \\
\text { level detection }\end{array}$ \\
\hline PEP-30-2-43 & $2 a$ & $\begin{array}{l}\text { Carryover of material } \\
\text { to the ventilation } \\
\text { system. }\end{array}$ & $\begin{array}{l}\text { High pressure on the vacuum } \\
\text { side or high pressure on the } \\
\text { air side results in overblow. }\end{array}$ & 3 & $\begin{array}{l}\text { PLC } \\
\text { PJM Level Detector } \\
\text { PJM Pressure } \\
\text { Detector } \\
\text { PJM Rack Air Supply } \\
\text { Valve } \\
\text { Feed Preparation tank } \\
\text { level detection }\end{array}$ \\
\hline PEP-30-3-02 & 3 & $\begin{array}{l}\text { Spill of simulant, } \\
\text { sodium hydroxide, } \\
\text { nitric acid/oxalic acid, } \\
\text { material, or inhibited } \\
\text { water from other tanks. }\end{array}$ & $\begin{array}{l}\text { No flow into the tank due to } \\
\text { leak in chemical addition or } \\
\text { transfer piping (flanges, etc.). }\end{array}$ & 4 & $\begin{array}{l}\text { PPE } \\
\text { Operating procedures } \\
\text { Facility secondary } \\
\text { containment } \\
\text { Ultrafiltration feed } \\
\text { tank level detection }\end{array}$ \\
\hline PEP-30-3-07 & 3 & $\begin{array}{l}\text { Spill of ultrafiltration } \\
\text { tank contents (simulant, } \\
\text { sodium hydroxide, } \\
\text { nitric acid/oxalic acid, } \\
\text { material, or inhibited } \\
\text { water from other } \\
\text { tanks). }\end{array}$ & $\begin{array}{l}\text { Structural integrity of } \\
\text { ultrafiltration feed tank } \\
\text { degraded, resulting in leak. }\end{array}$ & 1 & $\begin{array}{l}\text { Design of } \\
\text { ultrafiltration feed } \\
\text { tank to code }\end{array}$ \\
\hline PEP-30-3-08 & 3 & $\begin{array}{l}\text { Spill of ultrafiltration } \\
\text { tank contents (simulant, } \\
\text { sodium hydroxide, }\end{array}$ & $\begin{array}{l}\text { No flow out of the } \\
\text { ultrafiltration feed tank due to } \\
\text { leak in transfer piping/pumps. }\end{array}$ & 4 & $\begin{array}{l}\text { PPE } \\
\text { Operating procedures } \\
\text { Facility secondary }\end{array}$ \\
\hline
\end{tabular}


Table G-1. Safeguard Allocation

\begin{tabular}{|c|c|c|c|c|c|}
\hline $\begin{array}{l}\text { Scenario } \\
\text { Number }\end{array}$ & $\begin{array}{l}\text { No } \\
\text { de }\end{array}$ & Hazardous Condition & Cause & $\begin{array}{l}\text { Risk } \\
\text { Score }\end{array}$ & Credited Safeguards \\
\hline & & $\begin{array}{l}\text { nitric acid/oxalic acid, } \\
\text { material, or inhibited } \\
\text { water from other } \\
\text { tanks). }\end{array}$ & & & $\begin{array}{l}\text { containment } \\
\text { Ultrafiltration feed } \\
\text { tank level detection }\end{array}$ \\
\hline PEP-30-3-09 & 3 & $\begin{array}{l}\text { Carryover of material } \\
\text { to the ventilation } \\
\text { system. }\end{array}$ & $\begin{array}{l}\text { Lower-than-intended } \\
\text { ultrafiltration feed tank } \\
\text { volume. Tank level below } \\
\text { level of the PJMs results in } \\
\text { overblow. Material in the } \\
\text { vent line. }\end{array}$ & 3 & $\begin{array}{l}\text { PLC } \\
\text { PJM Level Detector } \\
\text { PJM Pressure } \\
\text { Detector } \\
\text { PJM Rack Air Supply } \\
\text { Valve } \\
\text { Ultrafiltration feed } \\
\text { tank level detection }\end{array}$ \\
\hline PEP-30-3-12 & 3 & $\begin{array}{l}\text { Spill of ultrafiltration } \\
\text { tank contents (simulant, } \\
\text { sodium hydroxide, } \\
\text { nitric acid/oxalic acid, } \\
\text { material, or inhibited } \\
\text { water from other } \\
\text { tanks). }\end{array}$ & $\begin{array}{l}\text { Too much material added to } \\
\text { the ultrafiltration tank } \\
\text { resulting in overflow of tank } \\
\text { to facility floor. }\end{array}$ & 4 & $\begin{array}{l}\text { PPE } \\
\text { Operating procedures } \\
\text { Facility secondary } \\
\text { containment } \\
\text { Ultrafiltration feed } \\
\text { tank level detection } \\
\text { and alarms } \\
\end{array}$ \\
\hline PEP-30-3-13 & 3 & $\begin{array}{l}\text { Incomplete/"out of } \\
\text { spec" batch of material. } \\
\text { Lost time for test. }\end{array}$ & $\begin{array}{l}\text { Too much material added to } \\
\text { the tank. High level in tank. }\end{array}$ & 3 & $\begin{array}{l}\text { Ultrafiltration feed } \\
\text { tank level detection } \\
\text { and alarms }\end{array}$ \\
\hline PEP-90-3-01 & 3 & $\begin{array}{l}\text { Spill of ultrafiltration } \\
\text { tank contents (simulant, } \\
\text { sodium hydroxide, } \\
\text { nitric acid/oxalic acid, } \\
\text { material, or inhibited } \\
\text { water from other } \\
\text { tanks). }\end{array}$ & $\begin{array}{l}\text { Ultafiltration pump flush } \\
\text { valves left open during pump } \\
\text { operation. Pump } \\
\text { ultrafiltration tank contents } \\
\text { into drain pan, overflowing } \\
\text { pan into facility. }\end{array}$ & 4 & $\begin{array}{l}\text { PPE } \\
\text { Facility secondary } \\
\text { containment } \\
\text { Operating procedures } \\
\text { Ultrafiltration feed } \\
\text { tank level detection }\end{array}$ \\
\hline PEP-30-3-15 & 3 & $\begin{array}{l}\text { Incomplete/"out of } \\
\text { spec" batch of material. } \\
\text { Lost time for test. }\end{array}$ & $\begin{array}{l}\text { Too little caustic added } \\
\text { resulting in contents too low } \\
\text { in caustic through } \\
\text { ultrafiltration system. }\end{array}$ & 3 & Operating procedures \\
\hline PEP-30-3-16 & 3 & $\begin{array}{l}\text { Incomplete/“"out of } \\
\text { spec" batch of material. } \\
\text { Lost time for test. }\end{array}$ & $\begin{array}{l}\text { Delivery of "out of spec" } \\
\text { caustic results in wrong batch } \\
\text { specs. }\end{array}$ & 3 & Operating procedures \\
\hline PEP-30-3-19 & 3 & $\begin{array}{l}\text { Failure of ultrafiltration } \\
\text { tank and spill of tank } \\
\text { contents (simulant, } \\
\text { sodium hydroxide, } \\
\text { nitric acid/oxalic acid, } \\
\text { material, or inhibited } \\
\text { water from other } \\
\text { tanks). }\end{array}$ & $\begin{array}{l}\text { High pressure in } \\
\text { ultrafiltration feed tank due to } \\
\text { ventilation blocked (closure } \\
\text { of damper, plugged) and } \\
\text { overfilling of tank. }\end{array}$ & 3 & $\begin{array}{l}\text { PSV on Ultrafiltration } \\
\text { Feed tank } \\
\text { Stop or bore through } \\
\text { on the damper to } \\
\text { prevent full closure. }\end{array}$ \\
\hline PEP-30-3-20 & 3 & $\begin{array}{l}\text { Failure of ultrafiltration } \\
\text { tank and spill of tank } \\
\text { contents (simulant, } \\
\text { sodium hydroxide, } \\
\text { nitric acid/oxalic acid, } \\
\text { material, or inhibited } \\
\text { water from other } \\
\text { tanks). }\end{array}$ & $\begin{array}{l}\text { High pressure in } \\
\text { ultrafiltration feed tank due to } \\
\text { ventilation blocked (closure } \\
\text { of damper, plugged), and the } \\
\text { tank overheats due to } \\
\text { temperature control system } \\
\text { failure. }\end{array}$ & 4 & $\begin{array}{l}\text { PSV on Ultrafiltration } \\
\text { Feed tank } \\
\text { Stop or bore through } \\
\text { on the damper to } \\
\text { prevent full closure. }\end{array}$ \\
\hline
\end{tabular}


Table G-1. Safeguard Allocation

\begin{tabular}{|c|c|c|c|c|c|}
\hline $\begin{array}{l}\text { Scenario } \\
\text { Number }\end{array}$ & $\begin{array}{l}\text { No } \\
\text { de }\end{array}$ & Hazardous Condition & Cause & $\begin{array}{l}\text { Risk } \\
\text { Score }\end{array}$ & Credited Safeguards \\
\hline PEP-30-3-28 & $3 b$ & $\begin{array}{l}\text { Incomplete/“out of } \\
\text { spec" batch of material. } \\
\text { Lost time for test. }\end{array}$ & $\begin{array}{l}\text { Add too much water to } \\
\text { ultrafiltration feed tank } \\
\text { (inhibited or condensate from } \\
\text { the sparger). }\end{array}$ & 3 & Operating procedures \\
\hline PEP-30-3-30 & $3 b$ & $\begin{array}{l}\text { Large steam leak } \\
\text { outside of tank. }\end{array}$ & $\begin{array}{l}\text { Failure of the steam system } \\
\text { upstream of the tank resulting } \\
\text { in large release of steam. }\end{array}$ & 2 & $\begin{array}{l}\text { Design of steam } \\
\text { system to code } \\
\text { Operating procedures }\end{array}$ \\
\hline PEP-30-3-31 & $3 b$ & $\begin{array}{l}\text { Small steam leak } \\
\text { outside of tank. }\end{array}$ & $\begin{array}{l}\text { Failure of the steam system } \\
\text { upstream of the tank resulting } \\
\text { in small release of steam. }\end{array}$ & 2 & $\begin{array}{l}\text { Design of steam } \\
\text { system to code } \\
\text { Operating procedures }\end{array}$ \\
\hline PEP-30-3-35 & $3 a$ & $\begin{array}{l}\text { Carryover of material } \\
\text { to the ventilation } \\
\text { system. }\end{array}$ & $\begin{array}{l}\text { High flow through the PJMs } \\
\text { results in overblow. }\end{array}$ & 3 & $\begin{array}{l}\text { PLC } \\
\text { PJM Level Detector } \\
\text { PJM Pressure } \\
\text { Detector } \\
\text { PJM Rack Air Supply } \\
\text { Valve } \\
\text { Ultrafiltration feed } \\
\text { tank level detection }\end{array}$ \\
\hline PEP-30-3-37 & $3 a$ & $\begin{array}{l}\text { Carryover of material } \\
\text { to the ventilation } \\
\text { system. }\end{array}$ & $\begin{array}{l}\text { High pressure on the vacuum } \\
\text { side or high pressure on the } \\
\text { air side results in overblow. }\end{array}$ & 3 & $\begin{array}{l}\text { PLC } \\
\text { PJM Level Detector } \\
\text { PJM Pressure } \\
\text { Detector } \\
\text { PJM Rack Air Supply } \\
\text { Valve } \\
\text { Ultrafiltration feed } \\
\text { tank level detection }\end{array}$ \\
\hline PEP-30-3-40 & 3 & $\begin{array}{l}\text { Internal vessel and } \\
\text { jacket is damaged, } \\
\text { releasing hot water. }\end{array}$ & $\begin{array}{l}\text { Blocked flow (closed valves) } \\
\text { to the chilled water jacket } \\
\text { resulting in over } \\
\text { pressurization of jacket. }\end{array}$ & 5 & $\begin{array}{l}\text { Operating procedures } \\
\text { PSV on chilled water } \\
\text { jacket }\end{array}$ \\
\hline PEP-30-3-41 & 3 & $\begin{array}{l}\text { Spill ultrafiltration tank } \\
\text { contents to the floor } \\
\text { (simulant, sodium } \\
\text { hydroxide, nitric } \\
\text { acid/oxalic acid, } \\
\text { material, or inhibited } \\
\text { water from other } \\
\text { tanks). }\end{array}$ & $\begin{array}{l}\text { Failure of the chilled water } \\
\text { system and leak the chilled } \\
\text { water into the ultrafiltration } \\
\text { tank, resulting in overflowing } \\
\text { the tank. }\end{array}$ & 2 & $\begin{array}{l}\text { PPE } \\
\text { Facility secondary } \\
\text { containment } \\
\text { Ultrafiltration feed } \\
\text { tank level detection } \\
\text { and alarms }\end{array}$ \\
\hline PEP-30-3-44 & 3 & $\begin{array}{l}\text { Carryover of material } \\
\text { into the ventilation } \\
\text { system and on to floor. }\end{array}$ & $\begin{array}{l}\text { Regulator failure results in } \\
\text { high pressure flow through } \\
\text { sparger. }\end{array}$ & 3 & Operating procedures \\
\hline PEP-30-4-01 & $4 \mathrm{~b}$ & $\begin{array}{l}\text { Contaminate/damage } \\
\text { the chilled water or } \\
\text { steam supply system. }\end{array}$ & $\begin{array}{l}\text { Failure in heat exchanger } \\
\text { causes leak of process } \\
\text { material into chilled water or } \\
\text { steam supply. Simulant } \\
\text { mixture plugs system. }\end{array}$ & 1 & Operating procedures \\
\hline PEP-30-4-02 & $4 \mathrm{~b}$ & $\begin{array}{l}\text { Contaminate/damage } \\
\text { the chilled water or } \\
\text { steam supply system. }\end{array}$ & $\begin{array}{l}\text { Failure of heat exchanger } \\
\text { causes leak of nitric acid into } \\
\text { chilled water supply or steam } \\
\text { supply. }\end{array}$ & 1 & Operating procedures \\
\hline
\end{tabular}


Table G-1. Safeguard Allocation

\begin{tabular}{|c|c|c|c|c|c|}
\hline $\begin{array}{l}\text { Scenario } \\
\text { Number }\end{array}$ & $\begin{array}{l}\text { No } \\
\text { de }\end{array}$ & Hazardous Condition & Cause & $\begin{array}{l}\text { Risk } \\
\text { Score } \\
\end{array}$ & Credited Safeguards \\
\hline PEP-30-4-05 & $4 \mathrm{~b}$ & $\begin{array}{l}\text { Damage the heat } \\
\text { exchanger loop } \\
\text { releasing heated } \\
\text { process material. }\end{array}$ & $\begin{array}{l}\text { Close isolation valves to heat } \\
\text { exchanger with continued } \\
\text { heating causes over } \\
\text { pressurization. }\end{array}$ & 6 & $\begin{array}{l}\text { Operating procedures } \\
\text { PSV on heat } \\
\text { exchanger }\end{array}$ \\
\hline PEP-30-4-06 & $4 \mathrm{~b}$ & $\begin{array}{l}\text { Large release steam to } \\
\text { the facility. }\end{array}$ & $\begin{array}{l}\text { Catastrophic failure of the } \\
\text { steam shell on the heat } \\
\text { exchanger results in large } \\
\text { release of steam. }\end{array}$ & 2 & $\begin{array}{l}\text { Design of steam shell } \\
\text { to code }\end{array}$ \\
\hline PEP-30-4-07 & $4 \mathrm{~b}$ & Collapse of steam shell. & $\begin{array}{l}\text { Steam present in the shell, } \\
\text { blocked off. Vacuum pulled } \\
\text { collapsing shell on cooling. }\end{array}$ & 2 & Operating procedures \\
\hline PEP-30-4-08 & $4 \mathrm{~b}$ & $\begin{array}{l}\text { Small release steam to } \\
\text { the facility. }\end{array}$ & $\begin{array}{l}\text { Structural failure of the steam } \\
\text { shell of exchanger. Small } \\
\text { leak to steam. }\end{array}$ & 1 & $\begin{array}{l}\text { Design of steam shell } \\
\text { to code }\end{array}$ \\
\hline PEP-30-4-09 & 4 & $\begin{array}{l}\text { Spill of simulant, } \\
\text { sodium hydroxide, } \\
\text { sodium permanganate, } \\
\text { nitric acid, or inhibited } \\
\text { water. }\end{array}$ & $\begin{array}{l}\text { No flow in the ultrafiltration } \\
\text { loop due to leak in chemical } \\
\text { addition or transfer piping } \\
\text { (flanges, etc.). }\end{array}$ & 4 & $\begin{array}{l}\text { PPE } \\
\text { Facility secondary } \\
\text { containment } \\
\text { Operating procedures }\end{array}$ \\
\hline PEP-30-4-19 & $4 a$ & $\begin{array}{l}\text { Leak of permeate and } \\
\text { pressurized air into } \\
\text { facility. }\end{array}$ & $\begin{array}{l}\text { Catastrophic failure of pulse } \\
\text { pot structure. Event happens } \\
\text { when pulsing. }\end{array}$ & 2 & $\begin{array}{l}\text { Design of pulse pot to } \\
\text { code } \\
\text { PSV on pulse pot }\end{array}$ \\
\hline PEP-30-4-20 & $4 a$ & $\begin{array}{l}\text { Small pressurized leak } \\
\text { of permeate to facility. }\end{array}$ & $\begin{array}{l}\text { Failure of pulse pot structure, } \\
\text { resulting in small leak. }\end{array}$ & 3 & $\begin{array}{l}\text { Design of pulse pot to } \\
\text { code }\end{array}$ \\
\hline PEP-30-4-21 & $4 a$ & $\begin{array}{l}\text { Release of reaction } \\
\text { aerosols out of nitric } \\
\text { acid tank to facility. }\end{array}$ & $\begin{array}{l}\text { Backflow of permeate back } \\
\text { into the nitric acid addition } \\
\text { system. Reaction in nitric } \\
\text { acid tank, releasing aerosols } \\
\text { out of vent. }\end{array}$ & 3 & Operating procedures \\
\hline PEP-90-4-05 & $4 a$ & $\begin{array}{l}\text { Spill of simulant, } \\
\text { sodium hydroxide, } \\
\text { sodium permanganate, } \\
\text { nitric acid, or inhibited } \\
\text { water. }\end{array}$ & $\begin{array}{l}\text { High pressure air causes } \\
\text { failure of filter assembly } \\
\text { releasing material to the } \\
\text { facility. }\end{array}$ & 1 & $\begin{array}{l}\text { Design of filter } \\
\text { assembly }\end{array}$ \\
\hline PEP-30-5-02 & 5 & $\begin{array}{l}\text { Spill of concentrated } \\
\text { slurry, inhibited water } \\
\text { to the facility floor. }\end{array}$ & $\begin{array}{l}\text { No flow into the tank due to } \\
\text { leak at the connection to the } \\
\text { flex hose connection to the } \\
\text { portable pump. }\end{array}$ & 5 & $\begin{array}{l}\text { PPE } \\
\text { Facility secondary } \\
\text { containment } \\
\text { Operating procedures } \\
\text { Slurry Concentrate } \\
\text { tank level detection } \\
\end{array}$ \\
\hline PEP-30-5-03 & 5 & $\begin{array}{l}\text { Spill of concentrated } \\
\text { slurry, inhibited water. }\end{array}$ & $\begin{array}{l}\text { No flow into the tank due to } \\
\text { leak in transfer piping } \\
\text { (flanges, etc.). }\end{array}$ & 4 & $\begin{array}{l}\text { PPE } \\
\text { Facility secondary } \\
\text { containment } \\
\text { Operating procedures } \\
\text { Slurry Concentrate } \\
\text { tank level detection } \\
\end{array}$ \\
\hline PEP-30-5-06 & 5 & $\begin{array}{l}\text { Spill of concentrated } \\
\text { slurry, inhibited water. }\end{array}$ & $\begin{array}{l}\text { Structural integrity of tank } \\
\text { degraded, resulting in leak. }\end{array}$ & 1 & $\begin{array}{l}\text { Design of Slurry } \\
\text { Concentrate tank to } \\
\text { code }\end{array}$ \\
\hline
\end{tabular}


Table G-1. Safeguard Allocation

\begin{tabular}{|c|c|c|c|c|c|}
\hline $\begin{array}{l}\text { Scenario } \\
\text { Number }\end{array}$ & $\begin{array}{l}\text { No } \\
\text { de }\end{array}$ & Hazardous Condition & Cause & $\begin{array}{l}\text { Risk } \\
\text { Score }\end{array}$ & Credited Safeguards \\
\hline PEP-30-5-07 & 5 & $\begin{array}{l}\text { Spill of concentrated } \\
\text { slurry, inhibited water. }\end{array}$ & $\begin{array}{l}\text { No flow to the receiving end } \\
\text { due to leak in transfer } \\
\text { piping/pumps. }\end{array}$ & 4 & $\begin{array}{l}\text { PPE } \\
\text { Facility secondary } \\
\text { containment } \\
\text { Operating procedures } \\
\text { Slurry Concentrate } \\
\text { tank level detection }\end{array}$ \\
\hline PEP-30-5-08 & 5 & $\begin{array}{l}\text { Spill of concentrated } \\
\text { slurry, inhibited water. }\end{array}$ & $\begin{array}{l}\text { No flow to receiving end due } \\
\text { to leak at the connection to } \\
\text { the simulant addition flex } \\
\text { hose. }\end{array}$ & 5 & $\begin{array}{l}\text { PPE } \\
\text { Facility secondary } \\
\text { containment } \\
\text { Operating procedures } \\
\text { Slurry Concentrate } \\
\text { tank level detection }\end{array}$ \\
\hline PEP-30-5-10 & 5 & $\begin{array}{l}\text { Overflow of tank } \\
\text { contents to ventilation } \\
\text { system, damaging fans, } \\
\text { exchanger, and blower. } \\
\text { Boil off of liquids } \\
\text { forming solids in vent } \\
\text { lines. }\end{array}$ & $\begin{array}{l}\text { Too much material added to } \\
\text { the tank, resulting overflow of } \\
\text { tank to the ventilation system. }\end{array}$ & 3 & $\begin{array}{l}\text { Slurry concentrate } \\
\text { tank level detection } \\
\text { and alarm }\end{array}$ \\
\hline PEP-30-5-11 & 5 & $\begin{array}{l}\text { Spill of concentrated } \\
\text { slurry, inhibited water } \\
\text { on to facility floor. }\end{array}$ & $\begin{array}{l}\text { Too much material added to } \\
\text { the tank with valves open, } \\
\text { resulting in spill out of } \\
\text { transfer line onto floor. }\end{array}$ & 4 & $\begin{array}{l}\text { PPE } \\
\text { Facility secondary } \\
\text { containment } \\
\text { Operating procedures } \\
\text { Slurry concentrate } \\
\text { tank level detection } \\
\text { and alarm } \\
\end{array}$ \\
\hline PEP-30-5-13 & 5 & $\begin{array}{l}\text { Spill of concentrated } \\
\text { slurry, inhibited water, } \\
\text { nitric acid, or simulant } \\
\text { on to facility floor. }\end{array}$ & $\begin{array}{l}\text { No flow to tank due to } \\
\text { portable pump discharge line } \\
\text { open transferring material } \\
\text { directly out open valve. }\end{array}$ & 5 & $\begin{array}{l}\text { PPE } \\
\text { Facility secondary } \\
\text { containment } \\
\text { Operating procedures } \\
\text { Slurry Concentrate } \\
\text { tank level detection }\end{array}$ \\
\hline PEP-30-5-14 & 5 & Operational upset. & $\begin{array}{l}\text { Too much material added to } \\
\text { the tank. High level in tank. }\end{array}$ & 3 & $\begin{array}{l}\text { Slurry Concentrate } \\
\text { tank level detection } \\
\text { and alarm }\end{array}$ \\
\hline PEP-30-5-15 & 5 & $\begin{array}{l}\text { Overflow of receipt } \\
\text { tank contents to } \\
\text { ventilation system, } \\
\text { damaging fans, } \\
\text { exchanger, and blower. } \\
\text { Boil off of liquids } \\
\text { forming solids in vent } \\
\text { lines. }\end{array}$ & $\begin{array}{l}\text { Backflow of material from } \\
\text { UFP-VSL-T62A to tank T27 } \\
\text { caused by valves open and } \\
\text { over fill T62A. }\end{array}$ & 2 & Operating procedures \\
\hline PEP-30-5-18 & 5 & $\begin{array}{l}\text { Failure of tank and spill } \\
\text { tank contents. }\end{array}$ & $\begin{array}{l}\text { High pressure in receipt tank } \\
\text { due to ventilation blocked } \\
\text { (closure of damper) and } \\
\text { overfilling of tank. }\end{array}$ & 6 & $\begin{array}{l}\text { PSV on Slurry } \\
\text { Concentrate tank } \\
\text { Stop or bore through } \\
\text { on the damper to } \\
\text { prevent full closure. }\end{array}$ \\
\hline PEP-30-6-03 & $6 \mathrm{a}$ & $\begin{array}{l}\text { Spill of permeate, } \\
\text { concentrated slurry, } \\
\text { simulant, sodium } \\
\text { hydroxide, sodium } \\
\end{array}$ & $\begin{array}{l}\text { No flow into the tank due to } \\
\text { leak at the connection to the } \\
\text { flex hose connection to the } \\
\text { portable pump. }\end{array}$ & 5 & $\begin{array}{l}\text { PPE } \\
\text { Facility secondary } \\
\text { containment } \\
\text { Operating procedures } \\
\end{array}$ \\
\hline
\end{tabular}


Table G-1. Safeguard Allocation

\begin{tabular}{|c|c|c|c|c|c|}
\hline $\begin{array}{l}\text { Scenario } \\
\text { Number }\end{array}$ & $\begin{array}{l}\text { No } \\
\text { de }\end{array}$ & Hazardous Condition & Cause & $\begin{array}{l}\text { Risk } \\
\text { Score }\end{array}$ & Credited Safeguards \\
\hline & & $\begin{array}{l}\text { permanganate, nitric } \\
\text { acid, or inhibited water } \\
\text { to the facility floor. }\end{array}$ & & & $\begin{array}{l}\text { Permeate receipt tank } \\
\text { level detection }\end{array}$ \\
\hline PEP-30-6-04 & $6 a$ & $\begin{array}{l}\text { Spill of permeate, } \\
\text { concentrated slurry, } \\
\text { simulant, sodium } \\
\text { hydroxide, sodium } \\
\text { permanganate, nitric } \\
\text { acid, or inhibited water } \\
\text { to the facility floor. }\end{array}$ & $\begin{array}{l}\text { No flow into the tank due to } \\
\text { leak in transfer piping } \\
\text { (flanges, etc.). }\end{array}$ & 4 & $\begin{array}{l}\text { PPE } \\
\text { Facility secondary } \\
\text { containment } \\
\text { Operating procedures } \\
\text { Permeate receipt tank } \\
\text { level detection }\end{array}$ \\
\hline PEP-30-6-07 & $6 a$ & $\begin{array}{l}\text { Spill of permeate, } \\
\text { concentrated slurry, } \\
\text { simulant, sodium } \\
\text { hydroxide, sodium } \\
\text { permanganate, nitric } \\
\text { acid, or inhibited water } \\
\text { to the facility floor. }\end{array}$ & $\begin{array}{l}\text { Structural integrity of tank } \\
\text { degraded, resulting in leak. }\end{array}$ & 1 & $\begin{array}{l}\text { Design of permeate } \\
\text { receipt tanks to code }\end{array}$ \\
\hline PEP-30-6-08 & $6 a$ & $\begin{array}{l}\text { Spill of permeate, } \\
\text { concentrated slurry, } \\
\text { simulant, sodium } \\
\text { hydroxide, sodium } \\
\text { permanganate, nitric } \\
\text { acid, or inhibited water } \\
\text { to the facility floor. }\end{array}$ & $\begin{array}{l}\text { No flow to the receiving tank } \\
\text { due to leak in transfer } \\
\text { piping/pumps. }\end{array}$ & 4 & $\begin{array}{l}\text { PPE } \\
\text { Facility secondary } \\
\text { containment } \\
\text { Operating procedures } \\
\text { Permeate receipt tank } \\
\text { level detection }\end{array}$ \\
\hline PEP-30-6-09 & $6 a$ & $\begin{array}{l}\text { Spill of permeate, } \\
\text { concentrated slurry, } \\
\text { simulant, sodium } \\
\text { hydroxide, sodium } \\
\text { permanganate, nitric } \\
\text { acid, or inhibited water } \\
\text { to the facility floor. }\end{array}$ & $\begin{array}{l}\text { No flow to receiving end due } \\
\text { to leak at the connection to } \\
\text { the simulant addition flex } \\
\text { hose. }\end{array}$ & 5 & $\begin{array}{l}\text { PPE } \\
\text { Facility secondary } \\
\text { containment } \\
\text { Operating procedures } \\
\text { Permeate receipt tank } \\
\text { level detection }\end{array}$ \\
\hline PEP-30-6-11 & $6 a$ & $\begin{array}{l}\text { Overflow of tank } \\
\text { contents to ventilation } \\
\text { system, damaging fans, } \\
\text { exchanger, and blower. } \\
\text { Boiling off liquids } \\
\text { forming solids in vent } \\
\text { lines. }\end{array}$ & $\begin{array}{l}\text { Too much material added to } \\
\text { the tank, resulting in overflow } \\
\text { of tank to the ventilation } \\
\text { system. }\end{array}$ & 3 & $\begin{array}{l}\text { Permeate receipt tank } \\
\text { level detection and } \\
\text { alarm }\end{array}$ \\
\hline PEP-30-6-12 & $6 a$ & $\begin{array}{l}\text { Spill of permeate, } \\
\text { concentrated slurry, } \\
\text { simulant, sodium } \\
\text { hydroxide, sodium } \\
\text { permanganate, nitric } \\
\text { acid, or inhibited water } \\
\text { to the facility floor. }\end{array}$ & $\begin{array}{l}\text { Too much material added to } \\
\text { the tank with valves open, } \\
\text { resulting in spill out of } \\
\text { transfer line onto floor. }\end{array}$ & 4 & $\begin{array}{l}\text { PPE } \\
\text { Facility secondary } \\
\text { containment } \\
\text { Operating procedures } \\
\text { Permeate receipt tank } \\
\text { level detection and } \\
\text { alarm }\end{array}$ \\
\hline PEP-30-6-14 & $6 a$ & $\begin{array}{l}\text { Spill of permeate, } \\
\text { concentrated slurry, } \\
\text { simulant, sodium } \\
\text { hydroxide, sodium } \\
\text { permanganate, nitric } \\
\text { acid, or inhibited water } \\
\text { to the facility floor }\end{array}$ & $\begin{array}{l}\text { Too much material added to } \\
\text { the tank with valves open, } \\
\text { resulting in backflow to } \\
\text { chemical addition tank } \\
\text { (sodium hydroxide, inhibited } \\
\text { water). }\end{array}$ & 3 & Operating procedures \\
\hline
\end{tabular}


Table G-1. Safeguard Allocation

\begin{tabular}{|c|c|c|c|c|c|}
\hline $\begin{array}{l}\text { Scenario } \\
\text { Number }\end{array}$ & $\begin{array}{l}\text { No } \\
\text { de }\end{array}$ & Hazardous Condition & Cause & $\begin{array}{l}\text { Risk } \\
\text { Score }\end{array}$ & Credited Safeguards \\
\hline & & $\begin{array}{l}\text { from the chemical } \\
\text { addition tank. }\end{array}$ & & & \\
\hline PEP-30-6-15 & $6 a$ & $\begin{array}{l}\text { Spill of permeate, } \\
\text { concentrated slurry, } \\
\text { simulant, sodium } \\
\text { hydroxide, sodium } \\
\text { permanganate, nitric } \\
\text { acid, or inhibited water } \\
\text { to the facility floor. }\end{array}$ & $\begin{array}{l}\text { No flow to receiving tank due } \\
\text { to portable pump discharge } \\
\text { line open, transferring } \\
\text { material directly out open } \\
\text { valve. }\end{array}$ & 5 & $\begin{array}{l}\text { PPE } \\
\text { Facility secondary } \\
\text { containment } \\
\text { Operating procedures } \\
\text { Permeate receipt tank } \\
\text { level detection }\end{array}$ \\
\hline PEP-30-6-17 & $6 a$ & $\begin{array}{l}\text { Overflow of receipt } \\
\text { tank contents to } \\
\text { ventilation system, } \\
\text { damaging fans, } \\
\text { exchanger, and blower. } \\
\text { Boiling off liquids } \\
\text { forming solids in vent } \\
\text { lines. }\end{array}$ & $\begin{array}{l}\text { Misroute of material from } \\
\text { source vessel to tank T62A } \\
\text { caused by valve misalignment } \\
\text { (intended to transfer to tank } \\
\text { other than T62A). Overflow } \\
\text { T62A. }\end{array}$ & 3 & Operating procedures \\
\hline PEP-30-6-20 & $6 a$ & $\begin{array}{l}\text { Spill of permeate, } \\
\text { concentrated slurry, } \\
\text { simulant, sodium } \\
\text { hydroxide, sodium } \\
\text { permanganate, nitric } \\
\text { acid, or inhibited water } \\
\text { to the facility floor. }\end{array}$ & $\begin{array}{l}\text { High pressure in receipt tank } \\
\text { due to ventilation blocked } \\
\text { (closure of damper) and } \\
\text { overfilling of tank. }\end{array}$ & 6 & $\begin{array}{l}\text { PSV on permeate } \\
\text { receipt tank } \\
\text { Stop or bore through } \\
\text { on the damper to } \\
\text { prevent full closure. }\end{array}$ \\
\hline PEP-30-6-23 & $6 a$ & $\begin{array}{l}\text { Damage equipment and } \\
\text { holdup of operation. }\end{array}$ & $\begin{array}{l}\text { Evaporate material to higher } \\
\text { concentration than wanted. } \\
\text { Let tank cool, material } \\
\text { solidifies in tank. Potential to } \\
\text { damage agitator. }\end{array}$ & 5 & $\begin{array}{l}\text { Operating procedures } \\
\text { Permeate evaporation } \\
\text { tank level detection } \\
\text { Permeate evaporation } \\
\text { tank temperature } \\
\text { indication }\end{array}$ \\
\hline PEP-30-6-24 & $6 a$ & $\begin{array}{l}\text { Failure of tank } \\
\text { releasing permeate, } \\
\text { concentrated slurry, } \\
\text { simulant, sodium } \\
\text { hydroxide, sodium } \\
\text { permanganate, nitric } \\
\text { acid, or inhibited water } \\
\text { to the facility. }\end{array}$ & $\begin{array}{l}\text { Over heat evaporator tank } \\
\text { with ventilation blocked } \\
\text { (closure of damper, plugging) } \\
\text { causes over pressurization } \\
\text { and damage to tank. }\end{array}$ & 6 & $\begin{array}{l}\text { PSV on permeate } \\
\text { receipt tank } \\
\text { Stop or bore through } \\
\text { on the damper to } \\
\text { prevent full closure. }\end{array}$ \\
\hline PEP-90-6-03 & $6 a$ & $\begin{array}{l}\text { Small release of steam } \\
\text { to the facility. }\end{array}$ & $\begin{array}{l}\text { Structural failure of the steam } \\
\text { jacket on collection tank. } \\
\text { Small leak of steam. }\end{array}$ & 1 & $\begin{array}{l}\text { Design of steam } \\
\text { jacket }\end{array}$ \\
\hline PEP-90-6-04 & $6 a$ & $\begin{array}{l}\text { Large release of steam } \\
\text { to the facility. }\end{array}$ & $\begin{array}{l}\text { Catastrophic failure of the } \\
\text { steam jacket on collection } \\
\text { tank results in large release of } \\
\text { steam. }\end{array}$ & 2 & $\begin{array}{l}\text { Design of steam } \\
\text { jacket }\end{array}$ \\
\hline PEP-90-6-05 & $6 a$ & $\begin{array}{l}\text { Collapse of steam } \\
\text { jacket. }\end{array}$ & $\begin{array}{l}\text { Steam present in the steam } \\
\text { jacket on the collection tank. } \\
\text { Block off jacket, add cold } \\
\text { water to refill. Vacuum } \\
\text { pulled collapsing jacket. }\end{array}$ & 2 & $\begin{array}{l}\text { Vacuum breaker on } \\
\text { steam jacket }\end{array}$ \\
\hline
\end{tabular}


Table G-1. Safeguard Allocation

\begin{tabular}{|c|c|c|c|c|c|}
\hline $\begin{array}{l}\text { Scenario } \\
\text { Number }\end{array}$ & $\begin{array}{l}\text { No } \\
\text { de }\end{array}$ & Hazardous Condition & Cause & $\begin{array}{l}\text { Risk } \\
\text { Score }\end{array}$ & Credited Safeguards \\
\hline PEP-90-6-01 & $6 a$ & $\begin{array}{l}\text { Overflow of tank } \\
\text { contents to ventilation } \\
\text { system, damaging fans, } \\
\text { exchanger, and blower. } \\
\text { Boiling off liquids } \\
\text { forming solids in vent } \\
\text { lines. }\end{array}$ & $\begin{array}{l}\text { Leak or failure of cooling line } \\
\text { inside permeate receipt tank } \\
\text { resulting in tank overflow } \\
\text { into the vessel ventilation } \\
\text { system. }\end{array}$ & 3 & $\begin{array}{l}\text { Permeate receipt tank } \\
\text { level detection and } \\
\text { alarm }\end{array}$ \\
\hline PEP-90-8-01 & 8 & $\begin{array}{l}\text { Large release of steam } \\
\text { outside and possible } \\
\text { natural gas fire. }\end{array}$ & $\begin{array}{l}\text { Structural failure of the } \\
\text { boiler. Release steam and } \\
\text { supply gas to outside. }\end{array}$ & 3 & Design boiler to code \\
\hline PEP-90-8-02 & 8 & $\begin{array}{l}\text { Large release of steam } \\
\text { outside and possible } \\
\text { natural gas fire. }\end{array}$ & $\begin{array}{l}\text { Overpressure in boiler results } \\
\text { in structural failure. Release } \\
\text { steam and supply gas to } \\
\text { outside. }\end{array}$ & 4 & PSV on boiler \\
\hline PEP-30-8-04 & 8 & $\begin{array}{l}\text { Large release of steam } \\
\text { in manned area. }\end{array}$ & $\begin{array}{l}\text { Catastrophic failure of system } \\
\text { within the facility. }\end{array}$ & 2 & $\begin{array}{l}\text { Design of steam } \\
\text { system to code } \\
\text { Operating procedures }\end{array}$ \\
\hline PEP-30-8-05 & 8 & $\begin{array}{l}\text { Small release of steam } \\
\text { in manned area. }\end{array}$ & $\begin{array}{l}\text { Minor failure of system } \\
\text { within the facility. }\end{array}$ & 3 & $\begin{array}{l}\text { Design of steam } \\
\text { system to code }\end{array}$ \\
\hline PEP-90-8-04 & 8 & $\begin{array}{l}\text { Natural gas fire outside } \\
\text { facility. }\end{array}$ & $\begin{array}{l}\text { Failure of boiler natural gas } \\
\text { supply results in fire. } \\
\text { Damage to facility structure. }\end{array}$ & 0 & $\begin{array}{l}\text { Natural gas system is } \\
\text { designed to code }\end{array}$ \\
\hline PEP-FN-8-01 & 8 & $\begin{array}{l}\text { Natural gas fire outside } \\
\text { facility. }\end{array}$ & $\begin{array}{l}\text { Natural gas supply line } \\
\text { damaged by impact with } \\
\text { load/vehicle, resulting in fire. }\end{array}$ & 2 & $\begin{array}{l}\text { Bollards around gas } \\
\text { connection }\end{array}$ \\
\hline PEP-30-9-02 & 9 & $\begin{array}{l}\text { Incomplete/“out of } \\
\text { spec" batch of material. } \\
\text { Lost time for test. }\end{array}$ & $\begin{array}{l}\text { Wrong material brought in to } \\
\text { SHR-VSL-T02 results in out } \\
\text { of specification caustic. }\end{array}$ & 3 & Operating procedures \\
\hline PEP-30-9-05 & 9 & $\begin{array}{l}\text { Spill of chemical to } \\
\text { facility. }\end{array}$ & Overfill the tank. & 4 & $\begin{array}{l}\text { Chemical tank level } \\
\text { detection and alarm }\end{array}$ \\
\hline PEP-30-9-07 & 9 & $\begin{array}{l}\text { Spill of chemical to the } \\
\text { facility. }\end{array}$ & $\begin{array}{l}\text { Failure of SHR-VSL-T01 } \\
\text { ( } 2 \mathrm{M} \text { caustic tank). }\end{array}$ & 1 & $\begin{array}{l}\text { Design of chemical } \\
\text { tanks }\end{array}$ \\
\hline PEP-FN-9-01 & 9 & $\begin{array}{l}\text { Spray of } 19 \mathrm{M} \text { caustic } \\
\text { to the facility. }\end{array}$ & $\begin{array}{l}\text { Small line failure downstream } \\
\text { of caustic metering pump in } \\
\text { the RO system. }\end{array}$ & 1 & $\begin{array}{l}\text { Operator rounds } \\
\text { (visual identification) }\end{array}$ \\
\hline PEP-30-11-04 & 11 & $\begin{array}{l}\text { Personnel injury due to } \\
\text { debris projectiles. }\end{array}$ & $\begin{array}{l}\text { Failure of the air compression } \\
\text { system outside of facility. }\end{array}$ & 1 & $\begin{array}{l}\text { Accumulator designed } \\
\text { to code }\end{array}$ \\
\hline PEP-90-11-01 & 11 & $\begin{array}{l}\text { Personnel injury due to } \\
\text { debris projectiles. }\end{array}$ & $\begin{array}{l}\text { Overpressure in pulse pot } \\
\text { compressed air system due to } \\
\text { amplifier pressures exceeding } \\
\text { design pressure ( } 482 \mathrm{psi}) \text {. }\end{array}$ & 3 & $\begin{array}{l}\text { PSV on pulse pots } \\
\text { PSV on compressed } \\
\text { air system }\end{array}$ \\
\hline PEP-30-12-01 & 12 & $\begin{array}{l}\text { Carryover of material } \\
\text { to the ventilation } \\
\text { system. }\end{array}$ & $\begin{array}{l}\text { No/low flow (failure) in } \\
\text { vacuum system. PJMs are } \\
\text { less effective, results in } \\
\text { overblow. }\end{array}$ & 3 & $\begin{array}{l}\text { PLC } \\
\text { PJM Level Detector } \\
\text { PJM Pressure } \\
\text { Detector } \\
\text { PJM Rack Air Supply } \\
\text { Valve }\end{array}$ \\
\hline PEP-30-12-03 & 12 & $\begin{array}{l}\text { Carryover of material } \\
\text { to the ventilation } \\
\text { system. }\end{array}$ & $\begin{array}{l}\text { Contamination/particulates in } \\
\text { the vacuum air system due to } \\
\text { the cyclone separator not } \\
\text { operating as expected. Plug }\end{array}$ & 3 & $\begin{array}{l}\text { PLC } \\
\text { PJM Level Detector } \\
\text { PJM Pressure } \\
\text { Detector }\end{array}$ \\
\hline
\end{tabular}


Table G-1. Safeguard Allocation

\begin{tabular}{|c|c|c|c|c|c|}
\hline $\begin{array}{l}\text { Scenario } \\
\text { Number }\end{array}$ & $\begin{array}{l}\text { No } \\
\text { de }\end{array}$ & Hazardous Condition & Cause & $\begin{array}{l}\text { Risk } \\
\text { Score }\end{array}$ & Credited Safeguards \\
\hline & & & $\begin{array}{l}\text { valves causing system to be } \\
\text { less effective. The effect of } \\
\text { operation of PJMs will result } \\
\text { in overblow. }\end{array}$ & & $\begin{array}{l}\text { PJM Rack Air Supply } \\
\text { Valve }\end{array}$ \\
\hline PEP-90-12-03 & 12 & $\begin{array}{l}\text { Carryover of material } \\
\text { to the ventilation } \\
\text { system and ultimately } \\
\text { vessel ventilation } \\
\text { system failure. }\end{array}$ & $\begin{array}{l}\text { High water level in separator } \\
\text { (Vac vessel T03) results in } \\
\text { overflow to vessel vent } \\
\text { header and into blower. Lose } \\
\text { ventilation capability and } \\
\text { water in system. }\end{array}$ & 3 & Operator rounds \\
\hline PEP-90-13-01 & 13 & $\begin{array}{l}\text { Personnel injury due to } \\
\text { burn. }\end{array}$ & $\begin{array}{l}\text { Material temperature too } \\
\text { high. Sample taken burns } \\
\text { personnel. }\end{array}$ & 2 & $\begin{array}{l}\text { PPE } \\
\text { Operating procedures }\end{array}$ \\
\hline PEP-90-13-02 & 13 & $\begin{array}{l}\text { Small release of } \\
\text { material to the facility. }\end{array}$ & $\begin{array}{l}\text { Sample valve inadvertently } \\
\text { left open. Leak of material to } \\
\text { the facility. }\end{array}$ & 3 & $\begin{array}{l}\text { PPE } \\
\text { Operating procedures }\end{array}$ \\
\hline PEP-90-13-04 & 13 & $\begin{array}{l}\text { Small release of } \\
\text { material to the facility. }\end{array}$ & $\begin{array}{l}\text { When charging vacuum } \\
\text { system, liquid accumulates in } \\
\text { vacuum pot. Prior to } \\
\text { sampling, pot is dropped } \\
\text { while being emptied, spilling } \\
\text { accumulated material (max } \\
2 \text { liters). }\end{array}$ & 3 & PPE \\
\hline PEP-30-A-01 & A1 & $\begin{array}{l}\text { Personnel injury due to } \\
\text { fall. }\end{array}$ & $\begin{array}{l}\text { Operator falls when climbing } \\
\text { stairs/ladder to get to upper } \\
\text { platform or when performing } \\
\text { operations on platform. }\end{array}$ & 5 & $\begin{array}{l}\text { Design of platform, } \\
\text { stairs, ladder (grating, } \\
\text { railings) } \\
\text { Lighting (normal and } \\
\text { facility emergency) } \\
\end{array}$ \\
\hline PEP-30-A-02 & A1 & $\begin{array}{l}\text { Personnel injury due to } \\
\text { impact. }\end{array}$ & $\begin{array}{l}\text { Operator working on lower } \\
\text { level runs into low hanging } \\
\text { pipe or other equipment. }\end{array}$ & 3 & $\begin{array}{l}\text { PPE (hard hats) } \\
\text { Lighting (normal and } \\
\text { facility emergency) }\end{array}$ \\
\hline PEP-30-A-03 & A1 & $\begin{array}{l}\text { Personnel injury due to } \\
\text { burn. }\end{array}$ & $\begin{array}{l}\text { Exposure of Operator to } \\
\text { heated surfaces (greater than } \\
212 \text { F), i.e., piping, tanks, etc. }\end{array}$ & 4 & $\begin{array}{l}\text { Insulation on piping } \\
\text { and tank sides } \\
\text { PPE (gloves) }\end{array}$ \\
\hline PEP-30-A-04 & A2 & $\begin{array}{l}\text { Personnel injury due to } \\
\text { impact. }\end{array}$ & $\begin{array}{l}\text { Operator drops portable pump } \\
\text { (or other tool) when moving } \\
\text { on to upper platform. Falls to } \\
\text { lower level. }\end{array}$ & 5 & $\begin{array}{l}\text { Design of platform, } \\
\text { stairs (grating, } \\
\text { railings, toe boards) } \\
\text { PPE (hard hats) }\end{array}$ \\
\hline PEP-30-A-06 & A4 & $\begin{array}{l}\text { Personnel injury due to } \\
\text { rotating equipment. }\end{array}$ & $\begin{array}{l}\text { Operator injured by rotating } \\
\text { equipment (at lower level } \\
\text { interaction with pumps) } \\
\text { during activities on platform } \\
\text { with agitator operating. }\end{array}$ & 3 & $\begin{array}{l}\text { Machine guards on } \\
\text { agitators and pumps }\end{array}$ \\
\hline PEP-30-A-07 & A4 & $\begin{array}{l}\text { Personnel injury due to } \\
\text { steam burn. }\end{array}$ & $\begin{array}{l}\text { Steam system temporarily } \\
\text { down (or upon start-up). } \\
\text { When restarting system, } \\
\text { condensate is present in lines, } \\
\text { resulting in water hammer. } \\
\text { System fails, releasing steam. }\end{array}$ & 6 & $\begin{array}{l}\text { Operating procedures } \\
\text { Design of steam } \\
\text { system to code }\end{array}$ \\
\hline
\end{tabular}


Table G-1. Safeguard Allocation

\begin{tabular}{|c|c|c|c|c|c|}
\hline $\begin{array}{l}\text { Scenario } \\
\text { Number }\end{array}$ & $\begin{array}{l}\text { No } \\
\text { de }\end{array}$ & Hazardous Condition & Cause & $\begin{array}{l}\text { Risk } \\
\text { Score }\end{array}$ & Credited Safeguards \\
\hline PEP-30-A-08 & A5 & Personnel heat stress. & $\begin{array}{l}\text { High temperatures in building } \\
\text { environment (high ambient } \\
\text { temperatures etc.) }\end{array}$ & 3 & $\begin{array}{l}\text { Building HVAC with } \\
\text { temperature } \\
\text { monitoring } \\
\text { Administrative } \\
\text { controls for stop work }\end{array}$ \\
\hline PEP-30-A-09 & A5 & $\begin{array}{l}\text { Personnel injury due to } \\
\text { tripping, falling. }\end{array}$ & $\begin{array}{l}\text { Loss of facility power when } \\
\text { facility manned. Hazard for } \\
\text { tripping, falling, etc. due to } \\
\text { darkness. Potential to fall } \\
\text { from elevated structures. }\end{array}$ & 5 & $\begin{array}{l}\text { Lighting (facility } \\
\text { emergency) }\end{array}$ \\
\hline PEP-30-A-10 & A5 & $\begin{array}{l}\text { Personnel injury due to } \\
\text { high noise levels. }\end{array}$ & $\begin{array}{l}\text { High noise levels from } \\
\text { equipment operation. }\end{array}$ & 3 & $\begin{array}{l}\text { PPE (hearing } \\
\text { protection) - if needed }\end{array}$ \\
\hline PEP-30-B-01 & $\mathrm{B} 1$ & $\begin{array}{l}\text { Facility fire. Personnel } \\
\text { injury due to vehicle } \\
\text { impact and/or fire. } \\
\text { Damage to facility due } \\
\text { to impact and fire. }\end{array}$ & $\begin{array}{l}\text { Vehicle collision impacts } \\
\text { facility, ruptures fuel tank, } \\
\text { resulting in fire adjacent to } \\
\text { facility. Interact with } \\
\text { chemicals being received } \\
\text { outside (maximum of } 2 \text { totes.) }\end{array}$ & 3 & $\begin{array}{l}\text { Fire alarm } \\
\text { Sprinkler system }\end{array}$ \\
\hline PEP-30-B-02 & $\mathrm{B} 1$ & $\begin{array}{l}\text { Personnel injury due to } \\
\text { impact. Damage to } \\
\text { facility structure. }\end{array}$ & $\begin{array}{l}\text { Vehicle collision impacts } \\
\text { facility and injures facility } \\
\text { personnel. }\end{array}$ & 4 & Speed limits (PNNL) \\
\hline PEP-30-B-03 & $\mathrm{B} 1$ & $\begin{array}{l}\text { Personnel injury due to } \\
\text { impact. Damage to } \\
\text { facility structure. }\end{array}$ & $\begin{array}{l}\text { Drop/swing crane load during } \\
\text { chemical/equipment off- } \\
\text { loading or moving } \\
\text { (replacement). Impact } \\
\text { facility and/or personnel. }\end{array}$ & 4 & $\begin{array}{l}\text { Hoisting, Rigging, } \\
\text { Forklifts, and Aerial } \\
\text { Lifts Requirements }\end{array}$ \\
\hline PEP-30-B-04 & $\mathrm{B} 1$ & $\begin{array}{l}\text { Personnel injury due to } \\
\text { exposure to chemicals. } \\
\text { Release of chemicals to } \\
\text { the environment. }\end{array}$ & $\begin{array}{l}\text { Drop forklift load, or hit load } \\
\text { with forklift, during chemical } \\
\text { off-loading/moving. Failure } \\
\text { of tote/drum releasing } \\
\text { chemicals. }\end{array}$ & 4 & $\begin{array}{l}\text { Hoisting, Rigging, } \\
\text { Forklifts, and Aerial } \\
\text { Lifts Requirements }\end{array}$ \\
\hline PEP-30-B-05 & $\mathrm{B} 1$ & $\begin{array}{l}\text { Personnel injury due to } \\
\text { impact. Damage to } \\
\text { facility structure. }\end{array}$ & $\begin{array}{l}\text { Drop forklift load onto } \\
\text { operator or hit with forklift } \\
\text { when off-loading or moving } \\
\text { (replacement) of chemicals/ } \\
\text { equipment. Potential for } \\
\text { impact to facility structure. }\end{array}$ & 4 & $\begin{array}{l}\text { Hoisting, Rigging, } \\
\text { Forklifts, and Aerial } \\
\text { Lifts Requirements }\end{array}$ \\
\hline PEP-30-B-06 & $\mathrm{B} 2$ & $\begin{array}{l}\text { Personnel injury due to } \\
\text { exposure to material. } \\
\text { Large release of } \\
\text { permeate or simulant to } \\
\text { ground. }\end{array}$ & $\begin{array}{l}\text { Failure to connect the flex } \\
\text { hose correctly to the tanker } \\
\text { truck (or failure of system) } \\
\text { during material transfer } \\
\text { (simulant in or permeate out). } \\
\text { Valves opened and truck } \\
\text { contents spilled to ground. }\end{array}$ & 4 & $\begin{array}{l}\text { Operating procedures } \\
\text { PPE } \\
\text { Safety showers/eye } \\
\text { wash }\end{array}$ \\
\hline PEP-30-B-07 & $\mathrm{B} 2$ & $\begin{array}{l}\text { Personnel injury due to } \\
\text { splash of material. } \\
\text { Small spill of permeate } \\
\text { or simulant to ground. }\end{array}$ & $\begin{array}{l}\text { Failure to connect the flex } \\
\text { hose correctly to the tanker } \\
\text { truck (or failure of system) } \\
\text { during material transfer } \\
\text { (simulant in or permeate out). } \\
\text { Spill of material outside. }\end{array}$ & 2 & $\begin{array}{l}\text { Operating procedures } \\
\text { PPE } \\
\text { Safety showers/eye } \\
\text { wash }\end{array}$ \\
\hline
\end{tabular}


Table G-1. Safeguard Allocation

\begin{tabular}{|c|c|c|c|c|c|}
\hline $\begin{array}{l}\text { Scenario } \\
\text { Number }\end{array}$ & $\begin{array}{l}\text { No } \\
\text { de }\end{array}$ & Hazardous Condition & Cause & $\begin{array}{l}\text { Risk } \\
\text { Score }\end{array}$ & Credited Safeguards \\
\hline PEP-30-B-08 & B3 & $\begin{array}{l}\text { Personnel injury due to } \\
\text { explosion/fire. } \\
\text { Damage to facility } \\
\text { from fire. }\end{array}$ & $\begin{array}{l}\text { Forklift propane tank fire } \\
\text { inside facility. }\end{array}$ & 2 & $\begin{array}{l}\text { Fire alarm } \\
\text { Sprinkler system }\end{array}$ \\
\hline PEP-30-C-01 & $\mathrm{C} 1$ & $\begin{array}{l}\text { Personnel injury due to } \\
\text { exposure to chemicals. } \\
\text { Spill of material inside } \\
\text { facility. }\end{array}$ & $\begin{array}{l}\text { During chemical receipt, add } \\
\text { wrong chemical into wrong } \\
\text { receipt tank (acid into caustic } \\
\text { or reverse). Chemical } \\
\text { reaction causes high heat, } \\
\text { tank failure, reaction aerosol } \\
\text { release. }\end{array}$ & 5 & $\begin{array}{l}\text { Incompatible fitting } \\
\text { design between } \\
\text { chemical types } \\
\text { Operating procedures }\end{array}$ \\
\hline PEP-30-C-02 & $\mathrm{C} 1$ & $\begin{array}{l}\text { Personnel injury due to } \\
\text { exposure to chemicals. } \\
\text { Spill of material inside } \\
\text { facility. }\end{array}$ & $\begin{array}{l}\text { Misvalving in chemical } \\
\text { addition system causes } \\
\text { misroute to open path. Spill } \\
\text { to the facility. }\end{array}$ & 4 & $\begin{array}{l}\text { Operating procedures } \\
\text { PPE }\end{array}$ \\
\hline PEP-30-C-04 & $\mathrm{C} 1$ & $\begin{array}{l}\text { Personnel injury due to } \\
\text { electrical shock. }\end{array}$ & $\begin{array}{l}\text { Personnel in contact with heat } \\
\text { trace system results in } \\
\text { electrical shock. }\end{array}$ & 5 & UL listed heat trace \\
\hline PEP-30-C-05 & $\mathrm{C} 3$ & $\begin{array}{l}\text { Personnel injury due to } \\
\text { exposure to chemicals. } \\
\text { Small spill of material } \\
\text { inside facility. }\end{array}$ & $\begin{array}{l}\text { Overfill sample container, } \\
\text { open valve with container } \\
\text { mis-positioned or missing, or } \\
\text { drop sample container. } \\
\text { Splash operator and spill to } \\
\text { facility. }\end{array}$ & 3 & $\begin{array}{l}\text { PPE } \\
\text { Operating procedures }\end{array}$ \\
\hline PEP-30-C-06 & $\mathrm{C} 3$ & $\begin{array}{l}\text { Personnel injury due to } \\
\text { exposure to chemicals. } \\
\text { Small spill of material } \\
\text { inside facility. }\end{array}$ & $\begin{array}{l}\text { Impact or damage to sample } \\
\text { carrier during transport } \\
\text { through the facility. Spill of } \\
\text { approx } 10 \text { samples. }\end{array}$ & 3 & $\begin{array}{l}\text { Operating procedures } \\
\text { PPE } \\
\text { Design of sample } \\
\text { container }\end{array}$ \\
\hline PEP-30-D-01 & D1 & $\begin{array}{l}\text { Personnel injury due to } \\
\text { burn. }\end{array}$ & $\begin{array}{l}\text { Contact with hot surfaces } \\
\text { (piping) during sample } \\
\text { acquisition. }\end{array}$ & 2 & $\begin{array}{l}\text { Insulation on piping } \\
\text { PPE (gloves) }\end{array}$ \\
\hline PEP-30-D-02 & D1 & $\begin{array}{l}\text { Personnel injury due to } \\
\text { exposure to process } \\
\text { material. Release of } \\
\text { material to the facility. }\end{array}$ & $\begin{array}{l}\text { Opening a sample port when } \\
\text { the line is pressurized results } \\
\text { in pressurized release of } \\
\text { process materials. }\end{array}$ & 3 & $\begin{array}{l}\text { Operating procedures } \\
\text { PPE (gloves, safety } \\
\text { glasses) }\end{array}$ \\
\hline PEP-30-D-03 & D1 & $\begin{array}{l}\text { Personnel injury due to } \\
\text { exposure to high } \\
\text { temperature process } \\
\text { material. }\end{array}$ & $\begin{array}{l}\text { Pull a sample of high } \\
\text { temperature material. Sample } \\
\text { container breaks from the } \\
\text { heat. }\end{array}$ & 2 & $\begin{array}{l}\text { Design of sample } \\
\text { container } \\
\text { PPE }\end{array}$ \\
\hline PEP-30-D-04 & D1 & $\begin{array}{l}\text { Personnel injury due to } \\
\text { burn. }\end{array}$ & $\begin{array}{l}\text { Pull a sample of high } \\
\text { temperature material. } \\
\text { Operator is burned by hot } \\
\text { temperature surface of sample } \\
\text { container. }\end{array}$ & 2 & $\begin{array}{l}\text { PPE (gloves, safety } \\
\text { glasses) }\end{array}$ \\
\hline PEP-FN-D-01 & D2 & $\begin{array}{l}\text { Personnel exposure to } \\
\text { hot chemicals. Small } \\
\text { release to facility. }\end{array}$ & $\begin{array}{l}\text { Spill of sample when } \\
\text { transferring within facility. }\end{array}$ & 2 & $\begin{array}{l}\text { Operating procedures } \\
\text { PPE } \\
\text { Design of sample } \\
\text { container }\end{array}$ \\
\hline PEP-FN-D-02 & D2 & $\begin{array}{l}\text { Personnel exposure to } \\
\text { hot chemicals. Small } \\
\text { release to facility. }\end{array}$ & $\begin{array}{l}\text { Mishandling of sample during } \\
\text { analysis activities exposes } \\
\text { personnel to chemicals. }\end{array}$ & 2 & $\begin{array}{l}\text { Operating procedures } \\
\text { PPE }\end{array}$ \\
\hline
\end{tabular}


Table G-1. Safeguard Allocation

\begin{tabular}{|c|c|c|c|c|c|}
\hline $\begin{array}{l}\text { Scenario } \\
\text { Number }\end{array}$ & $\begin{array}{l}\text { No } \\
\text { de }\end{array}$ & Hazardous Condition & Cause & $\begin{array}{l}\text { Risk } \\
\text { Score }\end{array}$ & Credited Safeguards \\
\hline PEP-FN-D-03 & D3 & $\begin{array}{l}\text { Small release of } \\
\text { material outside of } \\
\text { facility. }\end{array}$ & $\begin{array}{l}\text { Spill of sample tray when } \\
\text { transferring to archive area. }\end{array}$ & 2 & $\begin{array}{l}\text { Operating procedures } \\
\text { PPE } \\
\text { Design of sample } \\
\text { container }\end{array}$ \\
\hline PEP-FN-D-04 & D3 & $\begin{array}{l}\text { Release of material } \\
\text { outside facility. }\end{array}$ & $\begin{array}{l}\text { Vehicle collision with sample } \\
\text { archive area. }\end{array}$ & 0 & $\begin{array}{l}\text { Speed limits (PNNL) } \\
\text { Design of sample } \\
\text { cabinet (Conex) } \\
\text { Secondary } \\
\text { confinement on } \\
\text { sample archive } \\
\text { cabinet. }\end{array}$ \\
\hline PEP-FN-D-05 & D3 & $\begin{array}{l}\text { Release of material } \\
\text { outside facility. }\end{array}$ & $\begin{array}{l}\text { Extreme environmental } \\
\text { conditions. Sample } \\
\text { containers in cabinet fail. }\end{array}$ & 1 & $\begin{array}{l}\text { Design of sample } \\
\text { container } \\
\text { Secondary } \\
\text { confinement on } \\
\text { sample archive } \\
\text { cabinet. }\end{array}$ \\
\hline PEP-30-E-01 & E2 & $\begin{array}{l}\text { Personnel injury due to } \\
\text { exposure to process } \\
\text { material. Release of } \\
\text { material to the facility. }\end{array}$ & $\begin{array}{l}\text { Recovery actions to a plugged } \\
\text { line in system. Disconnect } \\
\text { flanges, removing valve } \\
\text { bodies, etc. Spill material to } \\
\text { the facility. }\end{array}$ & 4 & JHA and work plan \\
\hline PEP-30-E-02 & E2 & $\begin{array}{l}\text { Personnel injury due to } \\
\text { exposure to pressurized } \\
\text { process material. } \\
\text { Release of material to } \\
\text { the facility. }\end{array}$ & $\begin{array}{l}\text { Recovery actions to a plugged } \\
\text { line in system. Disconnect } \\
\text { flanges, removing valve } \\
\text { bodies, etc. When open, } \\
\text { system back pressure releases } \\
\text { material to the facility. }\end{array}$ & 4 & JHA and work plan \\
\hline PEP-30-E-03 & E2 & $\begin{array}{l}\text { Personnel injury due to } \\
\text { impact. }\end{array}$ & $\begin{array}{l}\text { Recovery actions to a plugged } \\
\text { line in system. Disconnect } \\
\text { flanges, removing valve } \\
\text { bodies, etc. Drop equipment } \\
\text { when moving (manually or } \\
\text { with crane). }\end{array}$ & 5 & JHA and work plan \\
\hline PEP-30-E-04 & E2 & $\begin{array}{l}\text { Personnel injury due to } \\
\text { steam burn. }\end{array}$ & $\begin{array}{l}\text { Recovery/repair actions } \\
\text { (plugged process line, } \\
\text { replacing ultrafilter, etc.). } \\
\text { Failure to de-energize the } \\
\text { steam system. When system } \\
\text { opened, release steam. }\end{array}$ & 5 & JHA and work plan \\
\hline PEP-30-E-05 & E2 & $\begin{array}{l}\text { Personnel injury due to } \\
\text { electrical shock. }\end{array}$ & $\begin{array}{l}\text { Recovery/repair actions } \\
\text { (plugged line in system, } \\
\text { replacing ultrafilter, etc.). } \\
\text { Failure to de-energize the } \\
\text { electrical system (heat trace). } \\
\text { Operator interacts with } \\
\text { system. }\end{array}$ & 5 & JHA and work plan \\
\hline PEP-30-E-06 & E2 & $\begin{array}{l}\text { Personnel injury due to } \\
\text { impact with missile. }\end{array}$ & $\begin{array}{l}\text { Recovery/repair actions } \\
\text { (plugged line in system, } \\
\text { replacing ultrafilter, etc.). } \\
\text { Failure to de-energize the } \\
\text { compressed air. Open }\end{array}$ & 5 & JHA and work plan \\
\hline
\end{tabular}


Table G-1. Safeguard Allocation

\begin{tabular}{|c|c|c|c|c|c|}
\hline $\begin{array}{l}\text { Scenario } \\
\text { Number }\end{array}$ & $\begin{array}{l}\text { No } \\
\text { de }\end{array}$ & Hazardous Condition & Cause & $\begin{array}{l}\text { Risk } \\
\text { Score }\end{array}$ & Credited Safeguards \\
\hline & & & $\begin{array}{l}\text { system, resulting in high } \\
\text { pressure release of air. Air } \\
\text { stream (300 psi) lifts debris. }\end{array}$ & & \\
\hline PEP-30-E-07 & E2 & $\begin{array}{l}\text { Personnel injury due to } \\
\text { steam burn. }\end{array}$ & $\begin{array}{l}\text { Recovery actions to a } \\
\text { contaminated ventilation } \\
\text { system (carry over of } \\
\text { material). Failure to de- } \\
\text { energize the steam system. } \\
\text { When system opened, steam } \\
\text { released. }\end{array}$ & 5 & JHA and work plan \\
\hline PEP-30-E-08 & E2 & $\begin{array}{l}\text { Personnel injury due to } \\
\text { electrical shock. }\end{array}$ & $\begin{array}{l}\text { Recovery actions to a } \\
\text { contaminated ventilation } \\
\text { system (carry over of } \\
\text { material). Failure to de- } \\
\text { energize the electrical system. } \\
\text { Operator interacts with } \\
\text { electrical system. }\end{array}$ & 5 & JHA and work plan \\
\hline PEP-30-E-09 & E2 & $\begin{array}{l}\text { Personnel injury due to } \\
\text { exposure to carry-over } \\
\text { material. Release of } \\
\text { material to the facility. }\end{array}$ & $\begin{array}{l}\text { Recovery actions to a } \\
\text { contaminated ventilation } \\
\text { system (carry-over of } \\
\text { material). Spill carryover } \\
\text { material when breaking open } \\
\text { the system. }\end{array}$ & 4 & JHA and work plan \\
\hline PEP-30-E-10 & E4 & $\begin{array}{l}\text { Personnel injury due to } \\
\text { exposure to process } \\
\text { material. Release of } \\
\text { material to the facility. }\end{array}$ & $\begin{array}{l}\text { Failure to drain system prior } \\
\text { to removing a failed ultrafilter } \\
\text { or other failed equipment. } \\
\text { Process material left in lines } \\
\text { spills to facility floor. }\end{array}$ & 4 & JHA and work plan \\
\hline PEP-30-E-11 & E4 & $\begin{array}{l}\text { Personnel injury due to } \\
\text { impact. }\end{array}$ & $\begin{array}{l}\text { Load drop from crane when } \\
\text { lifting failed filter/equipment } \\
\text { or loading replacement filter } \\
\text { into system. }\end{array}$ & 4 & JHA and work plan \\
\hline PEP-30-E-12 & E4 & $\begin{array}{l}\text { Personnel injury due to } \\
\text { impact. }\end{array}$ & $\begin{array}{l}\text { Swing crane load when lifting } \\
\text { failed filter or loading } \\
\text { replacement filter into } \\
\text { system. Impact with } \\
\text { personnel. }\end{array}$ & 4 & JHA and work plan \\
\hline PEP-30-E-13 & E4 & $\begin{array}{l}\text { Damage to nearby } \\
\text { equipment. }\end{array}$ & $\begin{array}{l}\text { Drop or swing crane load } \\
\text { when lifting failed } \\
\text { filter/equipment or loading } \\
\text { replacement filter into } \\
\text { system. Impact with nearby } \\
\text { equipment (steam line, } \\
\text { HVAC, heat exchanger, tank, } \\
\text { etc.) }\end{array}$ & 2 & JHA and work plan \\
\hline PEP-30-E-14 & E4 & $\begin{array}{l}\text { Personnel injury due to } \\
\text { tool operation. }\end{array}$ & $\begin{array}{l}\text { Operator error using power } \\
\text { tools causes personnel injury. }\end{array}$ & 3 & JHA and work plan \\
\hline PEP-30-F-01 & F1 & $\begin{array}{l}\text { Personnel injury due to } \\
\text { exposure to process } \\
\text { waste. }\end{array}$ & $\begin{array}{l}\text { Overfill drum/tote with } \\
\text { process waste. Spill material } \\
\text { inside facility. }\end{array}$ & 4 & $\begin{array}{l}\text { Operating procedures } \\
\text { PPE }\end{array}$ \\
\hline
\end{tabular}


Table G-1. Safeguard Allocation

\begin{tabular}{|c|c|c|c|c|c|}
\hline $\begin{array}{l}\text { Scenario } \\
\text { Number }\end{array}$ & $\begin{array}{l}\text { No } \\
\text { de }\end{array}$ & Hazardous Condition & Cause & $\begin{array}{l}\text { Risk } \\
\text { Score }\end{array}$ & Credited Safeguards \\
\hline PEP-30-F-02 & $\mathrm{F} 2$ & $\begin{array}{l}\text { Personnel injury due to } \\
\text { exposure to process } \\
\text { waste. }\end{array}$ & $\begin{array}{l}\text { Drop drum/tote filled with } \\
\text { process waste when moving } \\
\text { with forklift. Spill contents } \\
\text { inside facility. }\end{array}$ & 4 & $\begin{array}{l}\text { Hoisting, Rigging, } \\
\text { Forklifts, and Aerial } \\
\text { Lifts Requirements } \\
\text { PPE }\end{array}$ \\
\hline PEP-30-F-03 & F2 & $\begin{array}{l}\text { Personnel injury due to } \\
\text { exposure to process } \\
\text { waste. }\end{array}$ & $\begin{array}{l}\text { Damage drum/tote filled with } \\
\text { process waste with forklift. } \\
\text { Spill contents inside facility. }\end{array}$ & 4 & $\begin{array}{l}\text { Hoisting, Rigging, } \\
\text { Forklifts, and Aerial } \\
\text { Lifts Requirements } \\
\text { PPE }\end{array}$ \\
\hline PEP-30-F-04 & $\mathrm{F} 2$ & $\begin{array}{l}\text { Personnel injury due to } \\
\text { burn. }\end{array}$ & $\begin{array}{l}\text { Personnel exposure to high- } \\
\text { temperature surface due to } \\
\text { drum/tote filled with high- } \\
\text { temperature process waste. }\end{array}$ & 2 & $\begin{array}{l}\text { PPE (gloves, safety } \\
\text { glasses) } \\
\text { Operating procedures }\end{array}$ \\
\hline PEP-30-F-05 & $\mathrm{F} 2$ & $\begin{array}{l}\text { Personnel injury due to } \\
\text { exposure to process } \\
\text { waste. }\end{array}$ & $\begin{array}{l}\text { Drop drum/tote filled with } \\
\text { process waste when moving } \\
\text { over to the storage pad. Spill } \\
\text { material outside of the } \\
\text { facility. }\end{array}$ & 3 & $\begin{array}{l}\text { Hoisting, Rigging, } \\
\text { Forklifts, and Aerial } \\
\text { Lifts Requirements } \\
\text { PPE }\end{array}$ \\
\hline PEP-30-F-06 & F3 & $\begin{array}{l}\text { Personnel injury due to } \\
\text { exposure to process } \\
\text { waste. Spill of } \\
\text { drum/tote contents to } \\
\text { the environment. }\end{array}$ & $\begin{array}{l}\text { Vehicle collision with 90-day } \\
\text { storage pad loaded with } \\
\text { drums/totes. }\end{array}$ & 4 & $\begin{array}{l}\text { Speed limits (PNNL) } \\
\text { Restricted Access }\end{array}$ \\
\hline PEP-30-F-07 & F3 & $\begin{array}{l}\text { Personnel injury due to } \\
\text { exposure to process } \\
\text { waste. Fire on pad. } \\
\text { Spill of drum/tote } \\
\text { contents to the } \\
\text { environment. }\end{array}$ & $\begin{array}{l}\text { Vehicle collision with 90-day } \\
\text { storage pad loaded with } \\
\text { drums/totes. Fire results from } \\
\text { vehicle accident. }\end{array}$ & 3 & $\begin{array}{l}\text { Speed limits (PNNL) } \\
\text { Restricted Access }\end{array}$ \\
\hline PEP-30-F-08 & F3 & $\begin{array}{l}\text { Personnel injury due to } \\
\text { exposure to process } \\
\text { waste. Spill of } \\
\text { drum/tote contents on } \\
\text { to the pad. }\end{array}$ & $\begin{array}{l}\text { Low ambient temperatures. } \\
\text { Contents of drum/totes freeze } \\
\text { damaging container. Spill } \\
\text { contents when thaw. }\end{array}$ & 3 & $\begin{array}{l}\text { 90-day storage pad } \\
\text { secondary } \\
\text { containment }\end{array}$ \\
\hline PEP-30-F-09 & F3 & $\begin{array}{l}\text { Personnel injury due to } \\
\text { exposure of fumes. }\end{array}$ & $\begin{array}{l}\text { Addition nitric acid is stored } \\
\text { temporarily outside. Spill of } \\
\text { nitric acid due to various } \\
\text { causes. Nitric pool creates } \\
\text { fumes. }\end{array}$ & 4 & $\begin{array}{l}\text { Speed limits (PNNL) } \\
\text { Restricted Access } \\
\text { DOT containers for } \\
\text { Nitric }\end{array}$ \\
\hline
\end{tabular}




\section{Appendix $\mathbf{H}$}

\section{Peer Review Form}


TBP final

H.1 


\section{Distribution}

No. of

Copies

OFFSITE
No. of

Copies

ONSITE

15 Pacific Northwest National Laboratory

D. D. Bachand K3-52

G. H. Beeman K3-52

J.G.H. Geeting (5) P7-28

G. B. Josephson K9-69

D. E. Kurath P7-28

W. E. Lawrence K6-28

G. J. Sevigny P7-27

R. S. Sullivan K6-52

J. Young K6-52

Project File (2) P7-28

4 Bechtel National, Inc.

D. Alford H4-02

M. Lindholm H4-02

C. Musick H4-02

B. Stiver H4-02

1 Washington Division of URS Corporation John Truax MS4-B2

Distr. 1 